\section{The Evolution of Lithospheric Deformation and Crustal Structure from Continental Margins to Oceanic Spreading Centers}

by

\author{
MARK DIETRICH BEHN
}

B.S., Bates College, 1996

Submitted in partial fulfillment of the requirements for the degree of

Doctor of Philosophy

at the
MASSACHUSETTS INSTITUTE OF TECHNOLOGY
and the

WOODS HOLE OCEANOGRAPHIC INSTITUTION

June 2002

(C) 2002 Mark D. Behn. All rights reserved.

The author hereby grants to MIT and WHOI permission to reproduce and distribute publicly paper and electronic copies of this thesis document in whole or in part.

Author

Joint Program in Oceanography, Massachusetts Institute of Technology and Woods Hole Oceanographic Institution April 5, 2002

Certified by

Jian Lin

Associate Scientist, WHOI

Thesis Co-Supervisor

Certified by

Maria T. Zuber

Griswold Professor of Geophysics, MIT

Thesis Co-Supervisor

Accepted by

Timothy L. Grove

Chair, Joint Committee for Marine Geology and Geophysics 


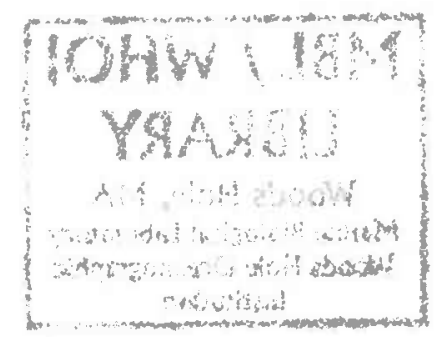




\title{
The Evolution of Lithospheric Deformation and Crustal Structure from Continental Margins to
}

\author{
Oceanic Spreading Centers
}

\author{
by \\ MARK DIETRICH BEHN \\ Submitted in partial fulfillment of the requirements for the \\ degree of Doctor of Philosophy \\ at the Massachusetts Institute of Technology \\ and the Woods Hole Oceanographic Institution \\ June, 2002
}

\begin{abstract}
This thesis investigates the evolution of lithospheric deformation and crustal structure from continental margins to mid-ocean ridges. The first part (Ch. 2) examines the style of segmentation along the U.S. East Coast Margin and investigates the relationship between incipient margin structure and segmentation at the modern Mid-Atlantic Ridge. The second part (Chs. 3-5) focuses on the mechanics of faulting in extending lithosphere. In Ch. 3 , I show that the incorporation of a strain-rate softening rheology in continuum models results in localized zones of high strain rate that are not imposed $a$ priori and develop in response to the rheology and boundary conditions. I then use this approach to quantify the effects of thermal state, crustal thickness, and crustal rheology on the predicted style of extension deformation. The mechanics of fault initiation and propagation along mid-ocean ridge segments is investigated in $\mathrm{Ch}$. 4. Two modes of fault development are identified: Mode $C$ faults that initiate near the center of a segment and Mode $E$ faults that initiate at the segment ends. Numerical results from Ch. 5 predict that over time scales longer than a typical earthquake cycle transform faults behave as zones of significant weakness. Furthermore, these models indicate that Mode $E$ faults formed at the inside-corner of a ridge-transform intersection will experience preferential growth relative to faults formed at the conjugate outside-corner due to their proximity to the weak transform zone. Finally, the last part of this thesis (Ch. 6) presents a new method to quantify the relationship between the seismic velocity and composition of igneous rocks. A direct relationship is derived to relate $\mathrm{V}_{\mathrm{p}}$ to major element composition and typical velocity-depth profiles are used to calculate compositional bounds for the lower continental, margin, and oceanic crust.
\end{abstract}


Thesis Co-Supervisor: Jian Lin

Title: Associate Scientist, WHOI

Thesis Co-Supervisor: Maria T. Zuber

Title: Griswold Professor of Geophysics, MIT

Thesis Committee:

Dr. Brian Tucholke, Senior Scientist, WHOI

Dr. Peter Kelemen, Senior Scientist, WHOI

Dr. Clark Burchfiel, Professor of Geology, MIT

Dr. Peter Clift, Associate Scientist, WHOI

Dr. Robert Detrick, Senior Scientist, WHOI (Chair) 


\section{Acknowledgments}

While my graduate career may not have been blessed by a Red Sox World Series Championship it was blessed by many fantastic friends, mentors, and role models. First and foremost, my advisors, Jian and Maria, deserve a tremendous "thank you" for their constant support, motivation, advice, and friendship over the last six years. Arriving in the Joint Program I found myself in the unique position of having two advisors who shared their own graduate experience together, and whose current research interests range from Martian climate to earthquake triggering on the San Andreas Fault. The result was an exceptionally compatible and complementary team that provided me with insightful advice on a vast range of scientific topics. Finally, and perhaps most importantly, I would like to thank Jian and Maria for always allowing me to pursue my own research interests, regardless of whether it was the path they themselves would have chosen.

I am grateful to the members of my thesis committee who provided invaluable insights into all aspects of my research over the last six years. I am indebted to Peter Kelemen for teaching me what little I know about geochemistry. Chapter 6 of this thesis arose from a project for Peter's class that I took in my second year as a student. Since that time Peter has been a great collaborator, supporter, and friend. Brian Tucholke's vast knowledge of midocean ridge tectonics (not to mention fly fishing) were critical in keeping my numerical models tied to reality. Peter Clift, with his perpetual upbeat attitude, made many of my trips to the field far more enjoyable and provided important insights into the evolution of continental margins. Clark Burchfiel took me on my first MIT field camp and provided a unique outlook on marine tectonics through his own expertise in continental geology. Bob Detrick was not only kind enough to serve as the Chair of my thesis committee, but also allowed me the opportunity to participate in the GPRIME cruise and has been a great scientific mentor. In addition, there are three other faculty members who should be thanked: Debbie Smith (my neighbor in the DIAL room), John Collins (who took me on my first cruise - with live explosives), and Greg Hirth (my rheologic advisor).

The number of fellow students from whom I have benefited over my graduate career is almost too many to count. At MIT I was lucky to have a great group of companions on the 5th floor: Laurent, Keli, Oded, Eliza, Jeff, Jun, Phil, Rebecca, Liangjun, Maureen, Margaret, Sergei, and Hugh. It is safe to say that my life would never have been as interesting without these individuals. Also at MIT I would like to thank the upstairs crowd: Eric, Jose, Arthur, Kevin, Simon, and Sinan. At WHOI, I was extremely fortunate to briefly overlap 
with Garrett Ito and Javier Escartín-these two "big brothers" have been great watchmates at sea and provided me with scientific and life advice countless times over the years. My days at Camp WHOI were enhanced both scientifically and socially by Carrie (my only classmate), Jeff, Mike, Margaret, Allegra, Sheri, Dave, Amy (D+M), Robyn, Fernanda, Astri, Matt, Mea, Rhea, Rose, Judith, Bill, Steve, Tom, Susie, Joe, Kelsey, Ben, Michael, Kirsten, Stefan, Alberto, and Gary. Finally, there are three individuals who deserve a special thanks for all the time we spent together both in and out of the office over the last six years: Frederik Simons for all our discussions of coherence, Belgian beers, and life after grad school; Jennifer Georgen, my companion in the DIAL room at all hours of the night (if not the day); and Noah Snyder who, appropriately enough, I first met at Fenway Park. Outside graduate school special thanks goes to Brad and Katherine (the original occupants of 26A Aberdeen Road) and James, John, and Ed (who have provided their unique outlook on the educational experience from other institutions). Finally, I would like to thank Liz for being so supportive over the last two years and knowing when to take my mind off science.

Last but not least I would like to express my deep gratitude toward my parents for helping me through all the years of my education - and of course without them I would not have made it to nearly as many Red Sox games.

Funding was provided by NASA through grants NAG5-3264, NAG5-4806, NAG5-11113, and NAG5-9143, and by a National Defense Science and Engineering Graduate Fellowship. 
to my grandfathers 


\section{Contents}

1 Introduction 13

1.1 Future Directions $\ldots \ldots \ldots \ldots \ldots \ldots \ldots \ldots$

2 Incipient segmentation of the oceanic lithosphere along the U.S. East Coast passive margin 19

2.1 Introduction . . . . . . . . . . . . . . . . . 20

2.2 Geologic Setting . . . . . . . . . . . . . . . . 23

2.3 Data Sources . . . . . . . . . . . . . . . . 26

2.3.1 Magnetic Field . . . . . . . . . . . . . . . 26

2.3.2 Bathymetry and Sediment Thickness . . . . . . . . . . 26

2.3 .3 Free-Air Gravity . . . . . . . . . . . . . . . . 30

2.3.4 Uncertainties in Offset Zone Locations . . . . . . . . . . . . 30

2.4 Gravity and Magnetic Anomalies . . . . . . . . . . . . . . . . 31

2.4.1 Comparison to Across Margin Seismic Lines . . . . . . . . . 32

2.4.2 Reduction-to-the-Pole Magnetic Anomalies . . . . . . . . . . . . 43

2.4.3 Peaks in R-T-P Magnetic and Isostatic Gravity Anomalies . . . . . 44

2.4.4 Conjugate West African Margin . . . . . . . . . . . . . 47

2.5 Along-Margin Segmentation and Implications . . . . . . . . . . . 47

2.5.1 Comparison to the Present-Day MAR Axis Segmentation . . . . . . 49

2.5.2 Relation to the Incipient Atlantic Offset Zones . . . . . . . . 51

2.5.3 Correlation With Across-Margin Isostatic Gravity Anomalies .. 52 
2.5.4 Kane and Atlantis Fracture Zones as Boundaries of Tectonic Corridors? . . . . . . . . . . . . . . . . . . 54

2.6 Conclusions . . . . . . . . . . . . . . . . 56

3 A continuum mechanics model for normal faulting using a strain-rate softening rheology $\quad 59$

3.1 Introduction . . . . . . . . . . . . . . 60

3.2 Model Setup . . . . . . . . . . . . . . . . . . . . 62

$3.2 .1 \quad$ Numerical Procedure . . . . . . . . . . . . . . . . . . 64

3.3 Numerical Results . . . . . . . . . . . . . . . . . . . . 68

3.3.1 Strain-Rate Softening Coefficient . . . . . . . . 68

3.3.2 Horizontal Temperature Gradient . . . . . . . . . . 72

3.3.3 Crustal Thickness . . . . . . . . . . . . . . . . . 74

3.3.4 Crustal Rheology . . . . . . . . . . . . . . . . 76

3.3.5 Geothermal Gradient . . . . . . . . . . . . . . . 80

3.4 Discussion . . . . . . . . . . . . . . . . . . . 80

3.4.1 Strain-rate vs. Strain Softening . . . . . . . . . . . 80

3.4.2 Crust- vs. Mantle-Dominated Deformation . . . . . . . . 82

3.4.3 Comparison to Natural Rifts . . . . . . . . . . . . . 84

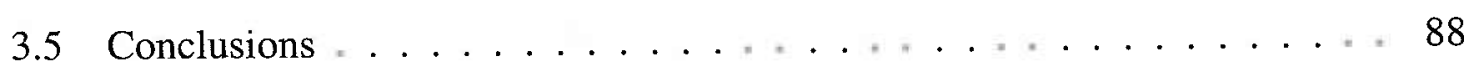

4 Mechanisms for normal fault development at mid-ocean ridges $\quad 89$

4.1 Introduction . . . . . . . . . . . . . . 90

4.2 Rheology of a Mid-Ocean Ridge Spreading Segment . . . . . . . . . 94

4.2.1 Young's Modulus and State of Stress . . . . . . . . . . . 94

4.2 .2 Yield Strength $\ldots \ldots \ldots \ldots \ldots \ldots \ldots$

4.3 Model Setup . . . . . . . . . . . . . . . . . . . . 102

4.4 Model Results . . . . . . . . . . . . . . . . . . . . . . . . 105

4.5 Discussion. . . . . . . . . . . . . . . . 111 
4.5.1 Observations of Mode E Faults . . . . . . . . . . . . . . 111

4.5.2 Factors Favoring the Generation of Mode E Faults . . . . . . . . 112

4.5.3 Fault Initiation at Fast-Spreading Ridges . . . . . . . . . . . . 118

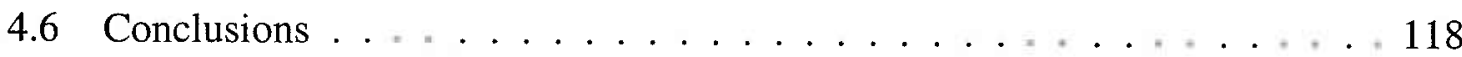

4.7 Thin-plate stress solution for linearly varying Young's modulus . . . . . . 120

4.7.1 Stress and Strain within the Plate . . . . . . . . . . . 120

4.7.2 Calculation of Initial Failure . . . . . . . . . . . . . . . 122

5 3-D stress interaction between oceanic transform faults and mid-ocean ridges 125

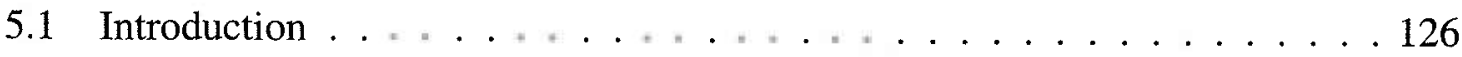

5.2 Stress State Near Oceanic Transform Faults . . . . . . . . . . . . . 130

5.2 .1 Model Setup . . . . . . . . . . . . . . . . . 131

5.2 .2 Numerical Results . . . . . . . . . . . . . . . . . 134

5.2.3 Transform Strength and Stress State at Inside Corners . . . . . . . . 143

5.3 Development of Normal Faults and Axial Topography at a Ridge-Transform

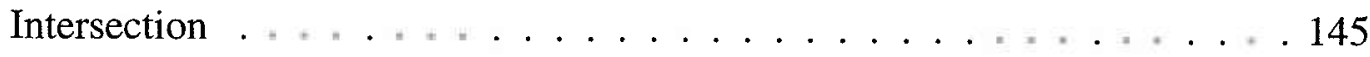

5.3 .1 Model Setup . . . . . . . . . . . . . . . . 146

5.3 .2 Numerical Results . . . . . . . . . . . . . . . 147

5.3.3 A Model for Enhanced Fault Growth at Inside-Corners . . . . . . 154

5.4 Conclusions . . . . . . . . . . . . . . . . . . . . 154

6 Relationship between Seismic Velocity and Igneous Rock Composition 157

6.1 Introduction . . . . . . . . . . . . . . . . . 158

6.2 Igneous Composition Space . . . . . . . . . . . . . . 160

6.2.1 Generation of Synthetic Bulk Compositions . . . . . . . . . . 162

6.3 Calculation of $V_{p}$ for Igneous Composition Space $\ldots \ldots 4$

6.3.1 Comparison to Laboratory Data . . . . . . . . . . . . . 168

6.4 Relating $V_{p}$ to Igneous Rock Composition . . . . . . . . . . . . . 172 
6.4.1 Direct Relationship between $V_{p}$ and Major Element Oxide Compo-

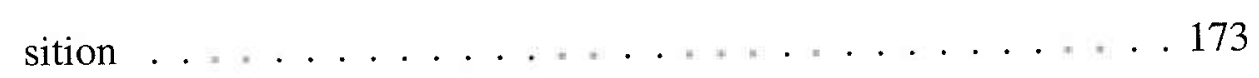

6.4.2 Relating $V_{p}$ to the Pressure and Fraction of Melting . . . . . . 176

6.4.3 Inverting Bulk Composition from Measurements of $V_{p} \ldots \ldots$. . . 178

6.5 Discussion . . . . . . . . . . . . . . . . . . 181

6.5 .1 Lower continental crust . . . . . . . . . . . . . . . 182

6.5 .2 Lower oceanic crust . . . . . . . . . . . . . . . 188

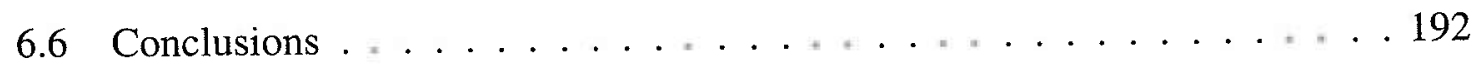

A Compositional Tables 


\section{Chapter 1}

\section{Introduction}

The opening of a new ocean basin is characterized by the progression from continental rifting to seafloor spreading and represents a fundamental feature of plate tectonics. In this thesis, I will examine a number of aspects of this process, focusing on the evolution of lithospheric deformation and crustal structure from continental margins to oceanic spreading centers. This thesis can be broadly divided into three parts. The first part (Chapter 2) examines the style segmentation along the U.S. East Coast margin and investigates its relationship to the segmentation observed at the modern Mid-Atlantic Ridge. The second part (Chapters 3-5) examines the mechanics of faulting in extending lithosphere, focusing on the sensitivity of fault development to the rheology and stress state of the brittle lithosphere and underlying ductile asthenosphere. Finally, in the third part (Chapter 6) I constrain the composition of the lower continental, margin, and oceanic crust by inverting seismic velocity data for major element chemistry. Below I provide a brief description of each chapter along with a summary of the fundamental conclusions presented therein.

Chapter 2 examines the 3-D structure of the continental-oceanic transition along the U.S. East Coast Margin using gravity, magnetics, and seismic data to better understand the evolution from continental rifting to oceanic spreading. Isostatic gravity anomalies show a corridor of high anomaly running along the margin, correlated with the location of the East Coast Magnetic Anomaly (ECMA), and situated close to the maximum thickness of high 
seismic velocity igneous crust as determined from seismic studies. Along-margin segmentation in both the ECMA and the isostatic gravity high are observed to have wavelengths of $\sim 100-150 \mathrm{~km}$, similar to the segmentation observed at the present day Mid-Atlantic Ridge. These results imply that segmentation is an important feature of margin development and that segmentation at mature oceanic spreading centers may be directly linked to segmentation during continental rifting. Moreover, the margin segmentation also reveals an additional $\sim 300-500 \mathrm{~km}$ wavelength that might be associated with super tectonic corridors of distinctive mantle properties beneath the Atlantic lithosphere.

The next three chapters deal with the mechanics of normal fault development in extending lithosphere. In Chapter 3, I investigate how a non-linear, strain-rate softening rheology can be incorporated into continuum models of lithospheric stretching to model the development of normal faults as a function of thermal structure and crustal rheology. Results of numerical experiments show that efficient strain-rate softening can result in localized zones of high strain rate, which develop in response to the rheology and boundary conditions and are not imposed a priori. Using this approach I quantify the horizontal temperature gradient necessary to focus deformation to the thinnest portion of the lithosphere. Further, I show that ductile flow in the lower crust is not sufficient to decouple deformation in the brittle upper crust from a strong upper mantle. Only in the extreme case, where stress accumulation in the upper crust is much greater than stress accumulation in the upper mantle do models predict that the upper crust will behave completely independently.

Chapters 4 and 5 focus specifically on faulting and stress state at a segmented oceanic spreading centers. Observed normal fault scarps at many mid-ocean ridge segments can be divided into two distinct groups: Mode $C$ (Center) faults that initiate near the center of a segment and propagate outward parallel to the ridge axis, and Mode $E$ faults that initiate at the segment ends and propagate inward toward the segment center. Mode $E$ faults are often rotated and typically cluster on inside-corner crust at the junction of a spreading segment and an active transform fault. In Chapter 4, I perform a thin-plate elastic-plastic stress analysis of a ridge segment to quantify the competing effects of along-axis variations in lithospheric strength and the elastic modulus of the plate in controlling fault initiation. 
Mode $C$ faults are predicted to develop at ridges where the along-axis variation in yield strength dominates the along-axis accumulation of stress due to changes in the elastic modulus. Conversely, Mode $E$ faults are predicted to form at ridges where stress accumulation toward the segment ends overcomes the high yield strength in these locations. This chapter also illustrates that transform faults must play an important role in generating the systematic concentration of Mode $E$ faults on inside-corner crust.

In Chapter 5, I conduct a series of 3-D boundary element calculations to further investigate the effects of transform faults on stress state and development of Mode $E$ faults at oceanic spreading centers. I find that on time scales longer than a typical earthquake cycle (i.e., $\sim 100$ years), transform faults behave as zones of significant weakness. Specifically, mechanical coupling of only $\sim 5 \%$ best explains the observed patterns of strike-slip and oblique normal faulting near a ridge-transform intersection. Further, I predict that extensional stresses will be suppressed at the inside-corner due to the shear along the transform resisting ridge-normal extension. This implies that the greater fault throw and enhanced microseismicity observed at inside-corners cannot be explained by elevated extensional stresses in these regions. Instead, I propose a model in which fault growth at segment ends is related to the interaction between normal faults and the neighboring transform. Boundary element calculations show that normal faults that develop at an inside-corner, adjacent to a weak transform fault, will experience preferential growth relative to faults forming at the OC away from the transform. This model provides a simple geometric explanation for enhanced fault growth and fault-related topography at the inside-corner of a ridge-transform intersection.

Finally, in Chapter 6 I perform an interdisciplinary study to quantify the relationship between seismic velocity and the composition of crustal rocks. This chapter addresses an important question for many seismological studies, namely when only the seismic velocity and the temperature and pressure conditions at a particular location are known, what is the full range of igneous compositions that are consistent with the seismic observations. I perform thermodynamic calculations of the equilibrating phase assemblages predicted for all igneous composition space at various pressure and temperature conditions. Seis- 
mic velocities for each assemblage are then estimated using the average of the upper and lower Hashin-Shtrikman bounds. The resultant velocities are used to derive a direct relationship between $V_{p}$ and major element composition valid to $\pm 0.12 \mathrm{~km} / \mathrm{s}$ for pressure and temperature conditions along a normal crustal geotherm in the depth range of $5-50 \mathrm{~km}$. The calculated velocities are also used to invert for major oxide chemistry as a function of seismic velocity. Compiling typical velocity-depth profiles for the lower continental and oceanic crust, I calculate compositional bounds for each of these geologic environments. I find that the acceptable compositional range for the lower continental crust is extremely large, ranging from basaltic to granitic compositions, and conclude that seismic velocity measurements alone are insufficient to provide fundamental constraints on the composition of the lower continental crust. In the case of oceanic crust, observed lower crustal velocities are $0.2-0.3 \mathrm{~km} / \mathrm{s}$ lower than velocities calculated based on the average bulk composition of gabbros in drill cores and exposed ophiolite sequences. I attribute this discrepancy to residual porosity at crustal depths less than $\sim 10 \mathrm{~km}$, and suggest caution when inferring mantle melting parameters from observed velocities in the lower oceanic crust.

\subsection{Future Directions}

As with any research, this thesis represents a mere stepping stone to future studies that will provide a clearer understanding of the active tectonic processes that shape the surface of the Earth. Looking ahead, I discuss several directions that I believe present the potential for exciting new discoveries in the field of continental and marine geodynamics.

The results of Chapter 2 strongly suggest that the processes leading to segmentation at the modern Mid-Atlantic Ridge may have already been active during continental rifting. However, while numerous dynamic models have been made to examine rifting in two dimensions, few have investigated the three-dimensional aspects of this problem. Is segementation controlled by deformation in the lithosphere or processes in the underlying asthenosphere? Furthermore, the results of Chapter 6 indicate that elevated mantle temperatures are not required to form the thick sequences of high-velocity igneous crust observed 
along the U.S. East Coast margin. This implies that "non-hotspot" models, in which large amounts of melt are fluxed across the solidus in the absence of a thermal anomaly, must be re-evaluated for the formation of volcanic margins. Specifically, secondary convection models may provide the opportunity for three-dimensional mantle upwelling in rifting environments [Korenaga and Jordan, 2002] that could be directly linked to incipient segmentation.

One of the fundamental questions in geodynamics regards how strain localizes in the lithosphere. At certain plate boundaries deformation is concentrated in a narrow zone just a few kilometers wide, while at others strain is distributed over hundreds of kilometers. On a regional scale, faults and shear zones represent first-order examples of strain localization. In Chapter 3, I examine the importance of strain-rate softening in localizing deformation in the brittle lithosphere. In addition to the rate dependence of frictional strength, there are other mechanisms that may lead to strain localization, such as cohesion loss, shear heating, and grain size reduction. However, while a number of recent studies (including this thesis) have examined the importance of individual localizing mechanisms, few are investigating the relative importance of multiple mechanisms in a self-consistent manner. Future studies will be critical to identify the relative importance of these various mechanisms and better understand the processes that govern the style of deformation in the lithosphere.

Finally, the rheologic layering of the lithosphere remains poorly constrained, both in oceanic and continental environments. For example, do the lower crust and upper mantle behave in a brittle or ductile fashion? At an even more basic level, is the simple yield strength envelope concept sufficient to explain the true behavior of the Earth? Recent advances in geodetic measurements through continuous GPS, strain-meter, and InSAR data are providing a better understanding of deformation in continental environments, although these technologies have not yet been adapted to the seafloor. Through the continued integration of geodynamic models with geological and geophysical data, future studies will be able to more accurately constrain the rheology of the lithosphere and apply this knowledge to processes such as rifting, orogenesis, post-glacial rebound, and the redistribution of stresses after a major earthquake. 


\title{
Chapter 2
}

\section{Incipient segmentation of the oceanic}

\section{lithosphere along the U.S. East Coast}

\section{passive margin*}

\begin{abstract}
Segmentation is a characteristic feature of seafloor spreading along the global mid-ocean ridge system. While segmentation of active spreading centers has been the focus of much recent research, the process by which a rifted continental margin develops into a segmented mid-ocean ridge is still poorly understood. In this study we investigate the segmentation character of the U.S. East Coast margin through a modeling study of the margin basement structure, magnetics, and gravity anomalies. The East Coast margin is of particular interest because it is one of several rifted continental margins that display thick sequences of high seismic velocity igneous crust, presumably formed during rifting. The East Coast Magnetic Anomaly (ECMA), a distinct total field magnetic high running offshore along the margin, is commonly located seaward of the thickest sections of the high-velocity crust and displays segmentation on length scales $(100-120 \mathrm{~km})$ similar to the segmentation observed at the Mid-Atlantic Ridge (MAR). Isostatic gravity anomalies were calculated by removing from free-air gravity the predicted effects of seafloor, sediments, and a crust-mantle model assuming local isostatic compensation. The resultant residuals show a corridor of high anomaly running along the margin, situated close to the maximum thickness of the
\end{abstract}

${ }^{*}$ Published as: M.D. Behn and J. Lin, Segmentation in gravity and magnetic anomalies along the U.S. East Coast passive margin: Implications for incipient structure of the oceanic lithosphere, J. Geophys. Res., v. 105 , pp. $25,769-25,790,2000$ 
high seismic velocity crust as determined from the two available seismic refraction lines. Reduction to the pole (R-T-P) of the total field magnetic anomaly shows that after the removal of skewness from the ECMA, the location of the isostatic gravity high is closely correlated to the ECMA. The isostatic gravity high is also segmented but in two distinct wave bands: $100-150 \mathrm{~km}$ and $300-500 \mathrm{~km}$. The short-wavelength $(100-150 \mathrm{~km})$ segmentation in the R-T-P magnetic and isostatic gravity anomalies is similar in wavelength to segmentation in magnetization and mantle Bouguer anomaly observed along the presentday MAR. The $300-500 \mathrm{~km}$ segmentation in the along-margin isostatic gravity anomaly is similar in length scale to both intermediate-wavelength tectonic segmentation observed in the South Atlantic and variations in lithospheric strength observed along the African margin. Furthermore, two of the intermediate-wavelength $(300-500 \mathrm{~km})$ isostatic gravity lows correspond to the early traces of the Kane and Atlantis fracture zones, suggesting that these two fracture zones may define boundaries of a single tectonic corridor in the North Atlantic. We hypothesize that the direct cause of the intermediate-wavelength segmentation may be along-margin variations in both the amount of underplated igneous crust and the strength of the lithosphere, although the relative importance of these two effects remains unresolved. Our results imply that segmentation is an important feature of margin development and that segmentation at mature oceanic spreading centers may be directly linked to segmentation during continental rifting.

\subsection{Introduction}

Passive or rifted-continental margins result from the rifting of continents during the formation of a new ocean basin. Rifted margins are often classified as either volcanic or nonvolcanic on the basis of the amount of volcanism that occurs during rifting [e.g., White and McKenzie, 1989]. Volcanic margins are characterized by thick sequences of high seismic velocity $(7.2-7.5 \mathrm{~km} / \mathrm{s}$ ) lower crust rocks (often referred to as underplating) and seaward dipping reflectors formed by the eruption and subsequent subsidence of volcanic flows during rifting. In contrast, nonvolcanic margins are generally associated with large rotated fault blocks, exposures of lower crustal and upper mantle rocks, and adjacent oceanic crust that is thinner than normal $(3-4 \mathrm{~km})$. Examples of volcanic margins include the Vøring Plateau [Mutter et al., 1984], Hatton Bank [White et al., 1987], and the Cuvier Margin [Hopper et al., 1992], while nonvolcanic margins have been observed off Iberia [Horsefield et al., 1993; Whitmarsh et al., 1998] and along the Exmouth Plateau [Hopper et al., 1992]. 
Early modeling efforts characterized rifting as a combination of stretching and thermal subsidence [e.g., McKenzie, 1978]. Although these models are capable of describing nonvolcanic margins, they are unable to account for the large sequences of igneous material found at volcanic margins. In an attempt to explain underplating, more recent models have focused on two principal mechanisms to form the high-velocity lower crust observed at volcanic margins: plume heads and secondary convection cells. Plume models require a thermal or chemical plume to be situated beneath the rifting crust [White and McKenzie, 1989]. The elevated mantle temperatures or chemical nature of the plume are hypothesized to cause enhanced decompression melting, leading to increased amounts of volcanism during passive rifting. Alternatively, Mutter et al. [1988] proposed that lateral temperature gradients, caused by the juxtaposition of hot upwelling mantle and cold continental crust during rifting, lead to the formation of secondary convection cells. These convection cells are hypothesized to generate active upwelling, which transports larger volumes of melt to the surface than would be allowed in a simple passive upwelling scenario [e.g., Boutilier and Keen, 1999].

Continental rifting is typically followed by the development of seafloor spreading along a margin. Spreading concentrates magmatic activity at a narrow ridge axis, which becomes the source of new oceanic crust. Mid-ocean ridge systems at slow spreading rates are documented to be highly three-dimensional in nature, with segmentation observed both at active spreading centers and in aged oceanic crust by the presence of fracture zones and off-axis traces of nontransform offsets [Grindlay et al., 1991; Sempéré et al., 1993; Tucholke and Lin, 1994] (see Figure 2-1). One topic of considerable research in recent years has been the relationship between segmentation and magma supply at the axis of a mid-ocean ridge. Whitehead et al. [1984] proposed that segmentation may be caused by gravitational instabilities that focus melt or mantle upwelling beneath spreading centers. This hypothesis has been supported by observations at slow spreading ridges, such as the Mid-Atlantic Ridge (MAR), where mantle Bouguer anomaly (MBA) lows are found at center of individual ridge segments [Kuo and Forsyth, 1988; Lin et al., 1990; Detrick et al., 1995]. These MBA "bull's-eye" patterns have been interpreted to represent focused mantle upwelling, which 


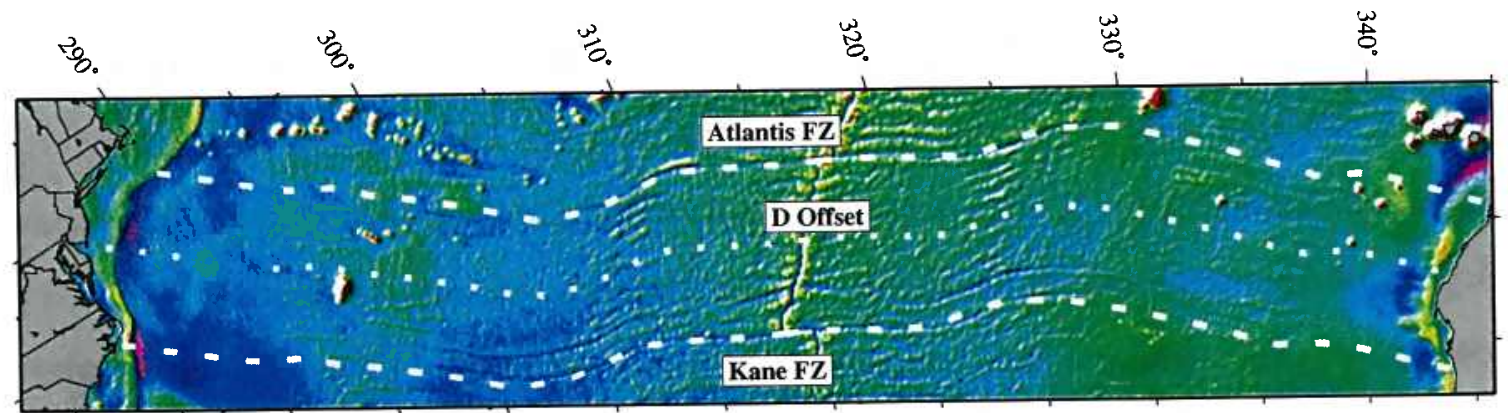

Figure 2-1: Free-air gravity of Sandwell and Smith [1997] illustrating the traces (dashed lines) of the Kane and Atlantis fracture zones from the U.S. East Coast margin to the conjugate West African margin. Also shown is a flow line (dotted line) projected from the intermediate-wavelength isostatic gravity low found near offset zone D between the Kane and Atlantis fracture zones.

result in increased crustal thickness at the center of ridge segments and thinner crust toward the segment ends [Kuo and Forsyth, 1988; Lin et al., 1990; Lin and Phipps Morgan, 1992]. On the other hand, magma supply at fast spreading ridges, such as the East Pacific Rise (EPR), appears to be more two-dimensional in nature with passive upwelling representing the major source of magma supply to the ridge-axis [Parmentier and Phipps Morgan, 1990; Lin and Phipps Morgan, 1992]. Alternatively, Magde and Sparks [1997] have recently suggested that crustal thickness variations along ridge segments may be caused by melt migration, as opposed to focused mantle upwellings. However, while many studies have examined magma supply at present-day ridges, few recent studies have addressed the possibility of segmentation in the supply of igneous underplated material to a continental margin preceding and during the initiation of seafloor spreading.

Studies in the Red Sea and West Africa suggest that passive margins may also exhibit three-dimensional structure. Cochran and Martinez [1988], for example, observed segmentation in both the marginal areas and axial valley of the Red Sea rift basin. In the Red Sea, "accommodation zones," or narrow regions that take up displacement between sets of fault blocks, extend perpendicularly across the passive margin and axial valley with an average spacing of $50-70 \mathrm{~km}$. Intrusions of basaltic magma are observed in the axial valley 
at the centers of the segments defined by these accommodation zones. Cochran and Martinez [1988] interpreted these observations to indicate that segmentation has been present in the Red Sea rift since its inception. In addition, intermediate-wavelength segmentation has been observed along the continental margin offshore of West Africa. Using gravity anomalies, Watts and Stewart [1998] showed a 350-400 km long weak zone extending along the Gabon margin of western Africa, and hypothesized that the entire West African margin may exhibit segmentation of this scale with alternating segments of strong and weak lithosphere. However, while these studies suggest segmentation in margin structure, they do not examine the relationship between igneous underplating and margin segmentation.

In this study we investigate the segmentation character of the U.S. East Coast margin, interpreted to be a strongly volcanic margin, through a modeling study of the basement structure, magnetics, and gravity anomalies. We find that the magnetic and isostatic gravity anomalies are segmented along the margin strike at various wavelengths. On the basis of modeling of these anomalies and comparisons with previous seismic studies we propose that the same source may be responsible for both the magnetic and gravity anomalies along the margin. Finally, we show that the segmentation of these anomalies is generally correlated with the location of early traces of identifiable Atlantic offsets. In particular, the observed short-wavelength $(100-150 \mathrm{~km})$ segmentation is comparable to the spacing of major nontransform offsets at the present-day MAR, while the anomaly lows in the intermediate-wavelength (300-500 km) segmentation correspond to the incipient location of the Kane, Atlantis, and D offset zones (see Figure 2-1).

\subsection{Geologic Setting}

The U.S. East Coast margin (see Figure 2-2) was formed during the rifting of Pangea in the Late Triassic and Early Jurassic [Klitgord et al., 1988]. Klitgord and Schouten [1986] suggested that seafloor spreading began around $175 \mathrm{Ma}$ and has continued through the present. Klitgord et al. [1988] defined three across-margin structural zones landward to seaward across the East Coast margin: thinned continental crust, rift stage or transitional 


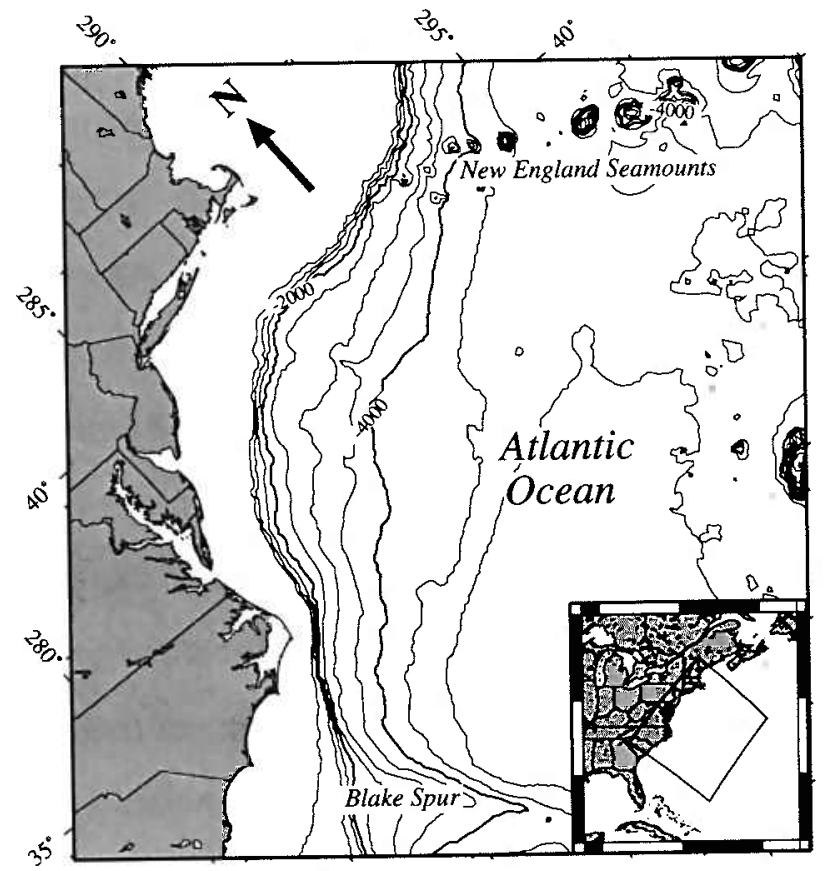

Figure 2-2: Location of the U.S. East Coast margin with bathymetry contoured at 500-m intervals. Inset shows an enlarged map of the U.S. East Coast used in Figure 2-3

crust, and oceanic crust. Within the rift stage crust, subsidence has led to the formation of a number of large Mesozoic-Cenozoic sedimentary basins, such as the Carolina Trough, Baltimore Canyon Trough, Blake Plateau Basin, and Georges Bank Basin.

Three distinct magnetic anomalies are observed running parallel to the East Coast margin: the Brunswick Magnetic Anomaly (BMA), the East Coast Magnetic Anomaly (ECMA), and the Blake Spur Magnetic Anomaly (BSMA). The BMA is the landwardmost of the three magnetic anomalies, extending north from Georgia to Cape Hatteras, where it intersects the ECMA. The offshore portion of the BMA marks the hinge zone along the landward edge of the marginal sedimentary basins [Klitgord et al., 1988]. The ECMA is a distinct magnetic high running parallel to the margin from the Blake Spur fracture zone (FZ) to Nova Scotia and is often taken to mark the continent-ocean boundary. Hypothesized sources for the ECMA include a highly magnetized ridge or intrabasement dike [Klitgord and Behrendt, 1979], faulting associated with rifting [Alsop and Talwani, 1984], an edge 
effect between transitional and oceanic crust [Hutchinson et al., 1983], and rift-related volcanics [Austin et al., 1990]. However, recent magnetics modeling of across-margin seismic refraction profiles strongly support the last mechanism as the source of the ECMA [Austin et al., 1990; Talwani et al., 1995]. The BSMA is the seawardmost of the three magnetic anomalies and has been associated with a ridge jump around 170 Ma [Klitgord and Schouten, 1986].

Over the last 2 decades a number of studies have focused on the volcanic character of the U.S. East Coast margin. Studies in the mid-1980s first suggested that the East Coast margin might be characterized by significant amounts of igneous underplating. Hinz [1981] and Klitgord et al. [1988] interpreted seaward dipping reflectors in multichannel seismic data from the Baltimore Canyon Trough and offshore of Georges Bank, respectively, as evidence for volcanism associated with rifting. Moreover, wide-angle seismic lines across the Baltimore Canyon Trough [LASE Study Group, 1986] and the Carolina Trough [Tréhu et al., 1989a] have found high lower crustal velocities $(7.2-7.5 \mathrm{~km} / \mathrm{s})$ beneath the transitional crust. Additional analyses of multichannel and wide-angle seismic data by Holbrook et al. [1994a, b] across the Carolina Trough and offshore of Virginia show $\sim 25 \mathrm{~km}$ thick sequences of high-velocity lower crust, interpreted as large intrusions of underplated igneous material. On the basis of these studies, Holbrook and Kelemen [1993] concluded that the U.S. East Coast margin is characterized by a $20-25 \mathrm{~km}$ thick section of high-velocity igneous material, emplaced at rifting and extending over $1000 \mathrm{~km}$ along the margin.

Invoking a plume model, White and McKenzie [1989] hypothesized that the Cape Verde hot spot, situated near the center of the East Coast margin, generated a broad $(\sim 1000 \mathrm{~km})$ thermal anomaly in the asthenosphere capable of producing the observed sequences of high-velocity material. Kelemen and Holbrook [1995] pointed out that a thermal anomaly centered on the Cape Verde hot spot should have generated a radially symmetrical pattern of igneous underplating, and yet no such systematic changes in the amount of underplating have been observed from seismic studies along the East Coast [Holbrook and Kelemen, 1993]. Therefore, as an alternative to the plume model, Kelemen and Holbrook [1995] proposed that the thick sequences of igneous crust were formed by a short-lived thermal 
anomaly in the upper mantle running along the entire margin, which dissipated rapidly after the initiation of normal seafloor spreading.

\subsection{Data Sources}

The goal of this study is to obtain a better understanding of the dynamics of magmatism during rifting along the U.S. East Coast through an analysis and joint interpretation of magnetic and isostatic gravity anomalies. In particular, we are interested in correlations between these two independent data sets that may provide new insights into the threedimensional nature of magmatism during rifting and the incipient structure of the oceanic lithosphere. The main types of data used in this study are addressed briefly in this section.

\subsubsection{Magnetic Field}

The magnetic field data used in this study were obtained from the Geological Survey of Canada (GSC) [Verhoef et al., 1996], providing $5 \mathrm{~km}$ spatial coverage across the U.S. East Coast margin (Figure 2-3). This data set consists of a compilation of shipboard magnetic surveys, aeromagnetic data, and previously gridded data sets from the period 19561992. These data have been correctly referenced to the International Geomagnetic Reference Field (IGRF) and filtered to remove anomalies with wavelengths $>400 \mathrm{~km}$. The final gridded data set represents the total field magnetic anomaly across the East Coast margin and clearly illustrates the ECMA running parallel to the margin and the high magnetic anomalies associated with the New England Seamounts (Figure 2-3).

\subsubsection{Bathymetry and Sediment Thickness}

In order to accurately correct for topographic effects in the free-air gravity, bathymetry of as high quality as possible is desired. The bathymetry data used in this study consist of ship track data obtained from the National Geophysical Data Center supplemented with ETOPO5 digital data between ship tracks. In order to give a greater weight to the higher 


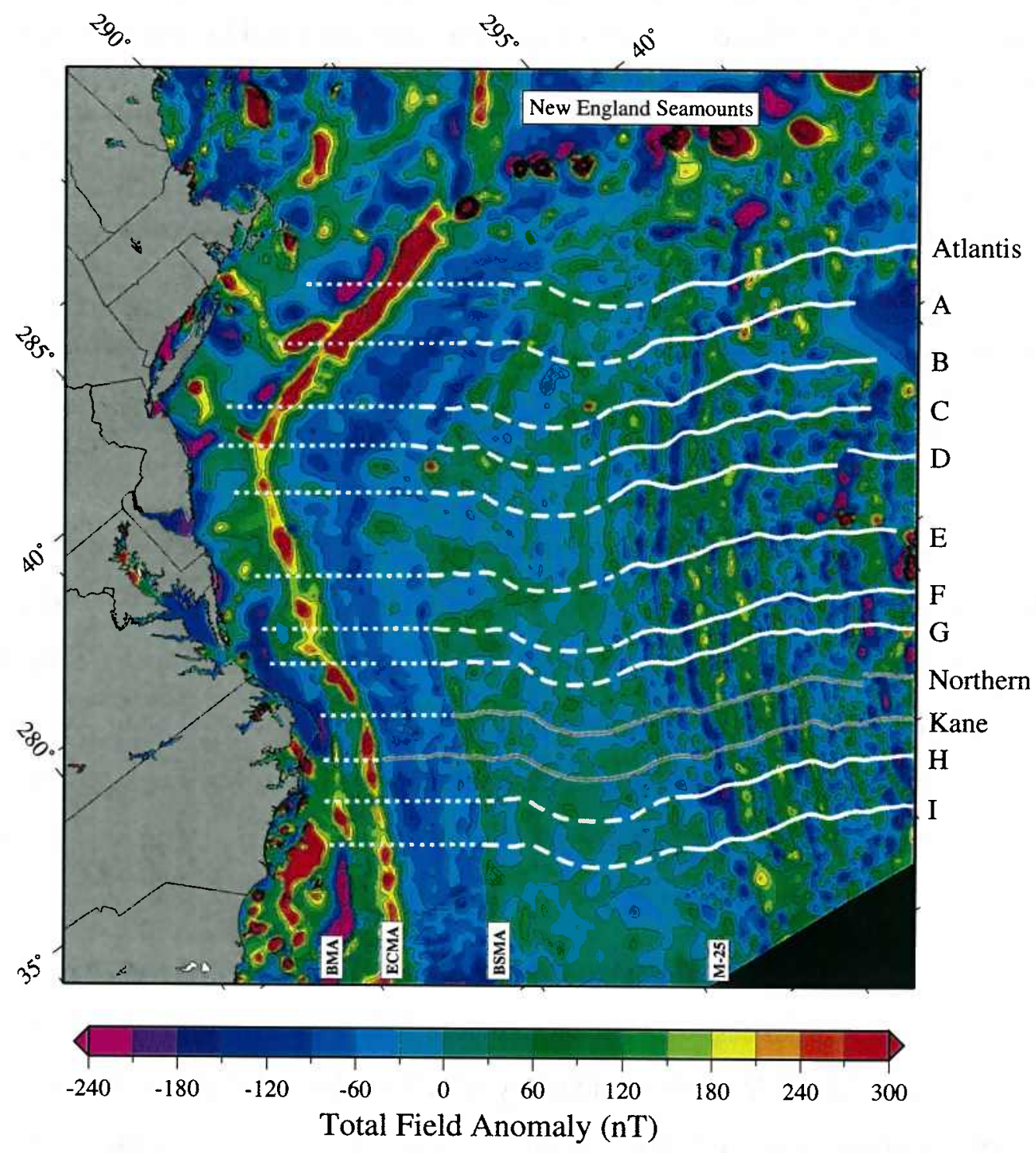

Figure 2-3: Total field magnetic anomaly from the Geological Survey of Canada [Verhoef et al., 1996]. Areas without adequate data control are masked in black. The segmented magnetic high running parallel to the margin is the East Coast Magnetic Anomaly (ECMA). Solid gray lines show the location of the Kane and Northern fracture zones as mapped by Tucholke and Schouten [1988] on the basis of a combination of basement structure and magnetic data. White lines illustrate the location of the offset zone traces identified in this study. Solid lines represent areas where the offset zone traces are constrained by offsets in magnetic lineations; dashed lines are used where the traces are primarily constrained from the flow lines of Tucholke and Schouten [1988]. The dotted lines are used to represent the high uncertainty in the location of the offset zone traces between the BSMA and the margin. Labels identifying the major offset zones (Atlantis, Kane, Northern, and A-I) are located in the right-hand margin. 
Figure 2-4: (facing page) (a) Shaded bathymetry of the U.S. East Coast, combining National Geophysical Data Center (NGDC) ship track data and ETOPO5 digital bathymetry data. In areas where NGDC ship tracks were located within 5 min of a grid point the NGDC data were used; in all other areas ETOPO5 data were used. Light black contour lines show sediment thickness from USGS seismic reflection grids, while thick black lines illustrate the extent of the USGS data coverage. (b) Free-air gravity derived from Sandwell and Smith's [1997] satellite gravity map. (c) Isostatic gravity anomaly, calculated by subtracting from the free-air gravity the effects of the water-sediment $\left(\Delta \rho=1300 \mathrm{~kg} / \mathrm{m}^{3}\right)$, sediment-crust $\left(\Delta \rho=400 \mathrm{~kg} / \mathrm{m}^{3}\right)$, and crust-mantle $\left(\Delta \rho=600 \mathrm{~kg} / \mathrm{m}^{3}\right)$ interfaces. The depth to the crust-mantle interface was calculated from the bathymetry and sediment thickness data assuming local Airy isostasy. See Figure 2-3 for notation on offset zone traces. (d) Reduced-to-the-pole (R-T-P) magnetic anomaly along the East Coast margin.

quality ship track data, all ETOPO5 data points within 5 min of a shiptrack were eliminated and the combined data set was then regridded on a $3 \mathrm{~min}$ grid to produce the bathymetry map shown in Figure 2-2 and Figure 2-4a. The bathymetry map clearly illustrates the three major physiographic features of the East Coast margin: the continental shelf, continental slope, and continental rise. Also shown in the bathymetry are the New England Seamount Chain located southeast of Cape Code, Massachusetts [Uchupi et al., 1970], and the Blake Plateau and Ridge located offshore of South Carolina [Dillon and Popenoe, 1988].

In addition to bathymetry, sediment thickness data were also acquired for the East Coast margin. The sediment thickness data shown in Figure 2-4a were extracted from 5 min digital sediment grids of the U.S. Geological Survey (USGS) [Klitgord et al., 1994; Hutchinson et al., 1996] and then resampled onto a 3 min grid. The USGS sediment thickness data are based on seismic reflection profiles, which have been verified against seismic refraction data and stratigraphic test wells where available. The USGS data are supplemented with data from Tucholke et al. [1982] in the region between $290^{\circ} \mathrm{E}$ and $295^{\circ} \mathrm{E}$. Sediment thickness is highly variable across the margin, reaching a maximum thickness of $12-14 \mathrm{~km}$ within the marginal sedimentary basins. Thus, in order to correct the gravity data for the effects of the low-density sediment layers, it is essential to have an accurate estimate of sediment thickness across the margin, such as the one available here along the U.S. East Coast. 


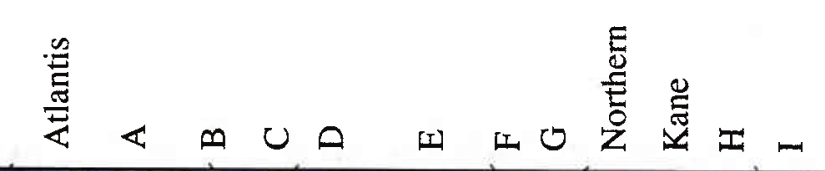
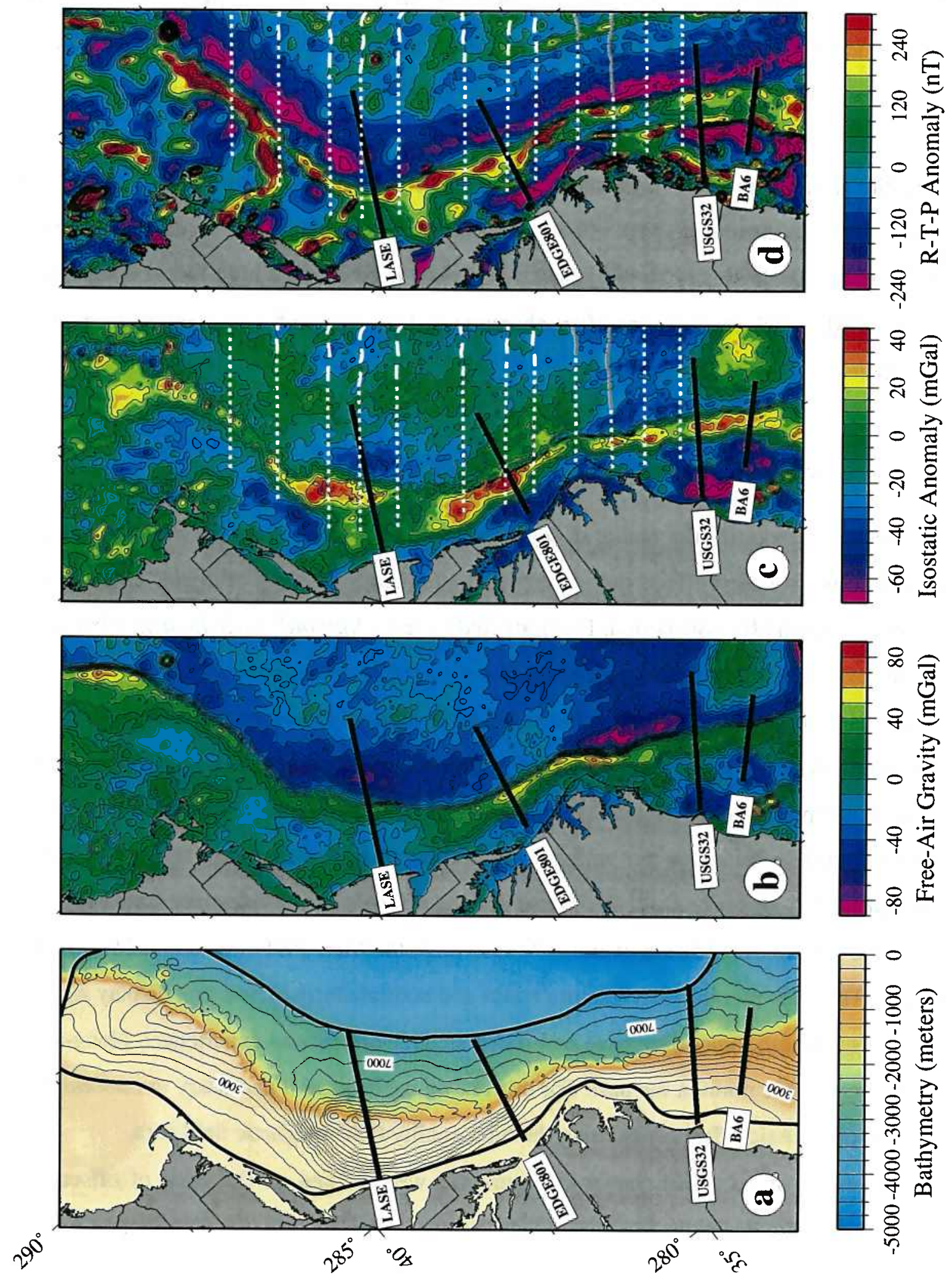


\subsubsection{Free-Air Gravity}

The free-air gravity map shown in Figure 2-4b was extracted from the 2 min satellite gravity map of Sandwell and Smith [1997]. A prominent high-low couple in the free-air gravity is situated over the transition between the continental shelf and slope, extending along the length of the margin. This feature, often referred to as the "continental edge effect," results from the abrupt transition from thick continental crust to thin oceanic crust. Watts and Marr [1995] have used this edge effect to characterize the strength of continental margins, showing that mechanically strong margins generally have a single high-low couple, while mechanically weak margins are often characterized by two high-low couples of smaller amplitude.

\subsubsection{Uncertainties in Offset Zone Locations}

In order to characterize the nature of segmentation in the lithosphere adjacent to the East Coast margin, we used offsets in the magnetic field data to identify the major offset zones present during the Jurassic period. Previous studies [e.g., Klitgord and Schouten, 1986] calculated average stage poles for the North American plate by examining seafloor magnetic lineations during periods of major changes in plate motion. However, the flow lines calculated from these stage poles and projected back from major offset zones at the present-day MAR axis represent only the average offset zone traces with time. It has been shown that small offset discontinuities are subject to migration along a plate boundary [e.g., Grindlay et al., 1991; Tucholke et al., 1997] and that the offset length of a fracture zone can vary from near zero to over $150 \mathrm{~km}$ over its life span (e.g., the Kane FZ [Tucholke and Schouten, 1988]). Thus it is possible that fracture zones and nontransform offsets that display significant offset length today may have had much smaller offsets and experienced along-axis migration at some point in the past. For this reason we deemed it necessary to reassess the location of Jurassic offset zones using the high quality GSC magnetic field data.

The major offset zones shown in Figure 2-3 were picked on the basis of offsets in seafloor magnetic lineations from the reduced-to-the-pole GSC data (see also Section 2.4.2). 
To insure that our identification of the major offsets was consistent with the known North American plate motion for the Jurassic, we used the location of the Kane and Northern fracture zones as determined by Tucholke and Schouten [1988] as a guide. Tucholke and Schouten [1988] used a combination of basement topography and magnetic anomalies to map the Kane and Northern fracture zone traces. More recent work along the conjugate West African margin by Verhoef et al. [1991] and Roest et al. [1992] further support the flow line picks of Tucholke and Schouten [1988], and thus we believe that their study provides the best available flow line constraint for the western North Atlantic.

Well-developed magnetic lineations are observed in the oceanic crust eastward of anomaly M-25 (see Figure 2-3). However, few clear lineations can be seen in crust formed during the Jurassic magnetic quiet zone [Vogt, 1973] between anomaly M-25 and the BSMA. This contrast in the amplitude of the observed magnetic lineations leads to less certainty in our identification of the offset zone traces in the region between M-25 and BSMA, where our picks are heavily dependent on flow lines of Tucholke and Schouten [1988]. West of the BSMA, there is even less constraint on the location of the offset zone traces. Using the limited amount of magnetic and seismic data available at the time, Klitgord and Schouten [1986] and Tucholke and Schouten [1988] observed the offset zone traces to trend approximately northwest across the BSMA until they intersect the ECMA. Thus, the highest uncertainty is assigned to the offset zone traces in the region between the BSMA and ECMA.

\subsection{Gravity and Magnetic Anomalies}

The free-air gravity signal comprises a number of effects, including those from seafloor topography, sediments, and the crust-mantle interface. In order to isolate local anomalies in the crust and mantle, we used the method described by Parker [1972] to extract the theoretical effects of the water-sediment $\left(\Delta \rho=1300 \mathrm{~kg} / \mathrm{m}^{3}\right)$, sediment-crust $(\Delta \rho=400$ $\left.\mathrm{kg} / \mathrm{m}^{3}\right)$, and crust-mantle $\left(\Delta \rho=600 \mathrm{~kg} / \mathrm{m}^{3}\right)$ interfaces. In areas of relatively little sedimentation and uniform oceanic crust, such as at mid-ocean ridges, a reference model of constant crustal thickness is often used, against which mantle Bouguer anomalies are calcu- 
lated by removing the effects of a reference model of uniform crustal thickness and density [Kuo and Forsyth, 1988; Lin et al., 1990]. However, in a margin setting, where the lateral change in crustal thickness from continental to oceanic crust can be up to a few tens of kilometers, a reference model of constant crustal thickness is no longer a good option [see Simpson et al., 1986]. Therefore, for the U.S. East Coast margin we calculated isostatic gravity anomalies against a reference model in which local Airy isostasy is assumed for the crustal column. The resultant isostatic gravity anomaly is shown in Figure 2-4c. We note that while using slightly different density parameters makes subtle changes to the detailed shape and magnitude of the isostatic anomaly, it does not alter the overall pattern of anomalies shown in Figure 2-4c. The isostatic anomaly calculated here compares well to that of Simpson et al. [1986], who calculated regional isostatic gravity anomalies for the continental United States. The largest discrepancies between the two studies are found in the regions where the sediment thickness is greatest (e.g., Baltimore Canyon Trough), illustrating the importance of incorporating the USGS sediment thickness data, which was unavailable to the study of Simpson et al. [1986].

\subsubsection{Comparison to Across Margin Seismic Lines}

Seismic studies represent the best tool currently available for imaging crustal structure. However, seismic transects are often limited to very few profiles across the area of interest. On the other hand, while gravity and magnetic maps are useful for revealing regionalscale lateral variations in anomalies, the interpretation of these anomalies is inherently nonunique. Therefore, in an attempt to better understand the implications of the observed geophysical anomalies along the U.S. East Coast margin, we examine in detail the isostatic gravity and magnetic anomalies along the two most recently available high-resolution seismic transects: EDGE-801 [Holbrook et al., 1994a] and BA-6 [Holbrook et al., 1994b] (Figures 2-5 and 2-6, respectively) as well as the Large Aperture Seismic Experiment (LASE) [LASE Study Group, 1986] and the USGS32 [Klitgord et al., 1988; Tréhu et al., 1989a] seismic lines (Figure 2-7). 
Along the four seismic transects examined, it appears that the peak in the isostatic gravity anomaly is situated directly over the region of high velocities in the lower crust (see Figures 2-5, 2-6, and 2-7). On the other hand, the position of the total field of the ECMA appears to be shifted seaward of the underplated material along the EDGE-801, BA-6, and USGS32. This seaward shift in the total field of the ECMA was observed by Holbrook and Kelemen [1993], who found the total field magnetics peak to be located between the thickest portion of the seaward dipping wedge and the landward limit of the oceanic crust. Holbrook et al. [1994a] and Talwani et al. [1995] have modeled the spatial correlation between the total field magnetics and high seismic velocities and suggested that the igneous rocks emplaced during rifting may act as the source for the ECMA.

Along the LASE line (Figures 2-7a and 2-7b), the relationship between the peak of the isostatic gravity anomaly and the total field of the ECMA is reversed from the other three seismic lines, with the isostatic gravity high located slightly seaward of the ECMA. One potential explanation for this seaward shift in the isostatic gravity high is related to the high-density carbonate reef identified between 120 and $160 \mathrm{~km}$ on the LASE line [Tréhu et al., 1989b]. Furthermore, the high-velocity lower crust along the LASE line appears to be broader in horizontal extent than along the three other seismic lines.

We carried out simple forward models to illustrate the relationship between igneous crust of high seismic velocity in its underplated section and the resulting gravity and magnetic anomalies. Figure 2-8 shows the isostatic gravity anomaly and magnetic anomalies associated with a simplified crustal density model for an underplated margin. This model reveals an isostatic anomaly of 50-60 mGal situated directly over the region of high velocity and density in the lower crustal rocks. The total field magnetic anomaly was calculated in the manner of Plouff [1976] with a Curie depth of $20 \mathrm{~km}$. Induced magnetization from the present-day field (inclination of $68^{\circ}$, declination of $-13^{\circ}$, field strength of $50,000 \mathrm{nT}$ ) was assumed for the crustal block, while remanent magnetization in the direction of the Jurassic field (inclination of $46^{\circ}$, declination of $-2.2^{\circ}$ [Austin et al., 1990]) was used for the seaward dipping reflector sequence situated above the underplated section. Talwani et al. [1995] argued convincingly that the ECMA must be generated by remanent magnetization 

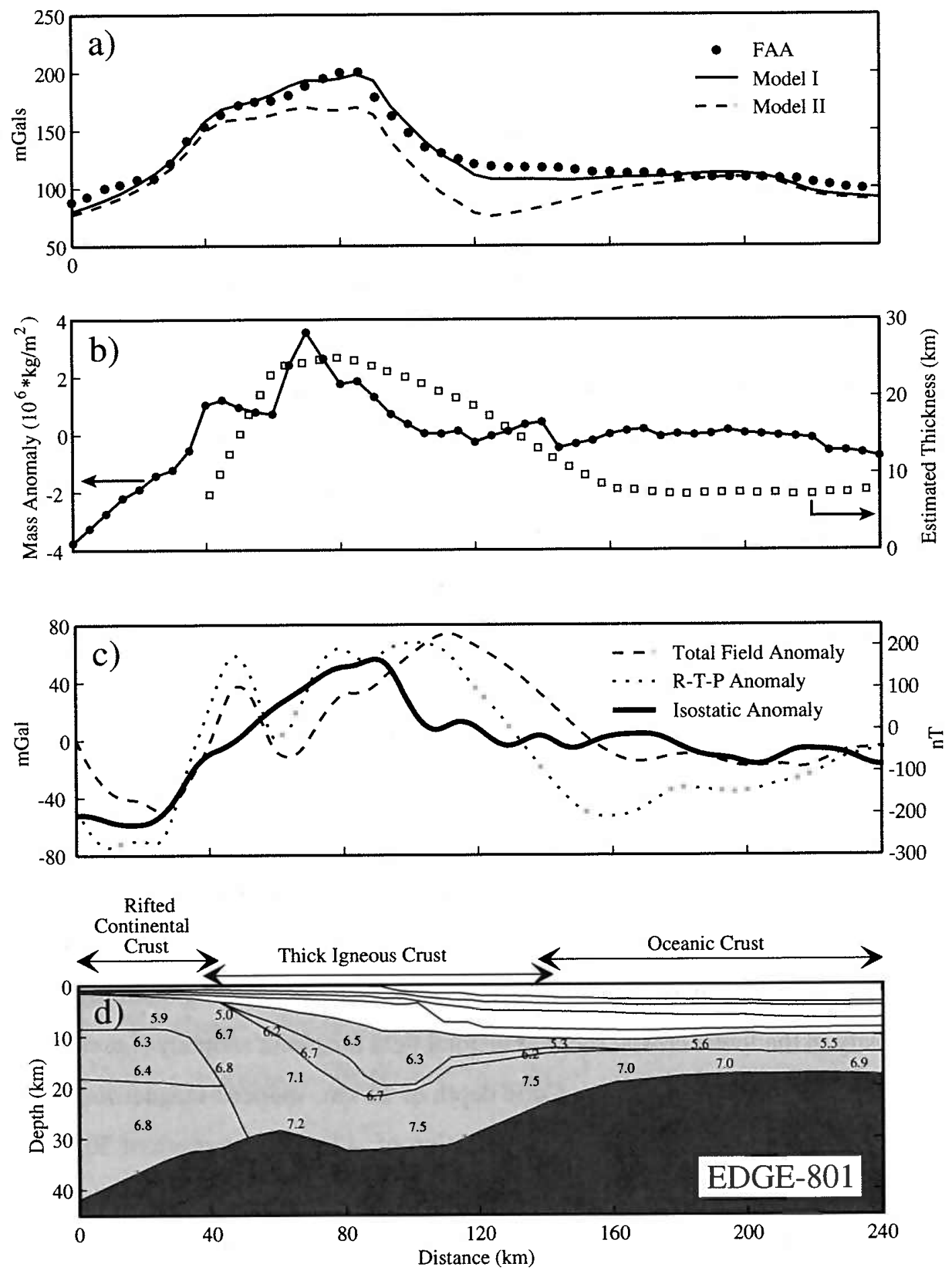
Figure 2-5: (facing page) Comparison of the EDGE-801 seismic line [Holbrook et al., 1994a] to observed geophysical anomalies. (a) Observed free-air gravity (FAA) (solid circles) versus modeled gravity. Model I is based on the density structure of Holbrook et al. [1994a], while model II uses a reduced lower crustal density of $3030 \mathrm{~kg} / \mathrm{m}^{3}$. Note that without the crustal density structure of Holbrook et al. [1994a] it would be difficult to reproduce the observed free-air gravity without assigning unreasonable densities to the upper crust. (b) Mass anomaly (solid circles) that results from the density structure of Holbrook et al. [1994a]. The relative mass anomaly is calculated by integrating the mass with depth in individual columns along the seismic profile and comparing to a reference model for normal oceanic crust. Open squares represent the estimated thickness of emplaced igneous rocks by Holbrook and Kelemen [1993]. (c) Isostatic gravity, total field magnetic, and reducedto-pole (R-T-P) magnetic anomalies sampled along the profile. (d) Crustal velocity model along the EDGE-801 line adapted from Holbrook et al. [1994a]. Note the good correlation between the isostatic mass anomaly in Figure $2-5 \mathrm{~b}$ and the calculated isostatic gravity anomaly and R-T-P magnetic anomaly in Figure 2-5c. Moreover, the maximum isostatic anomaly appears to be located directly over the region of thick, high-velocity lower crust.

of volcanic flows produced by subaerial seafloor spreading. They based their assumption on the very high remanent magnetization of volcanic rocks from Ocean Drilling Program (ODP) Hole 642, and the fact that if the ECMA were due to induced magnetization, the value of susceptibility would be unacceptably large. However, we note that given the similarity in inclination and declination of the present-day and Jurassic fields, modeling the seaward dipping reflector sequence with only an induced component would produce nearly identical anomalies to those produced by remanent magnetization.

Similar to the magnetic modeling of Talwani et al. [1995], we assume that oceanic crust seaward of the seaward dipping reflector sequence to have zero effective magnetization. This assumption is based on the observation of small amplitude of magnetic anomalies in the oceanic crust lying within the magnetic quiet zone between the ECMA and BSMA. Talwani et al. [1995] hypothesize that this magnetic quiet zone is most likely caused by the juxtaposition of normally and reversely polarized subhorizontal lava flows emplaced subaerially during a period of slow seafloor spreading. The magnetic model shown in Figure 2-8 illustrates that as observed in the EDGE-801, BA-6, and USGS32 seismic lines, the peak in the total field anomaly is shifted seaward of the underplated section. Thus both the isostatic gravity high and total field magnetic high can be explained from the same 

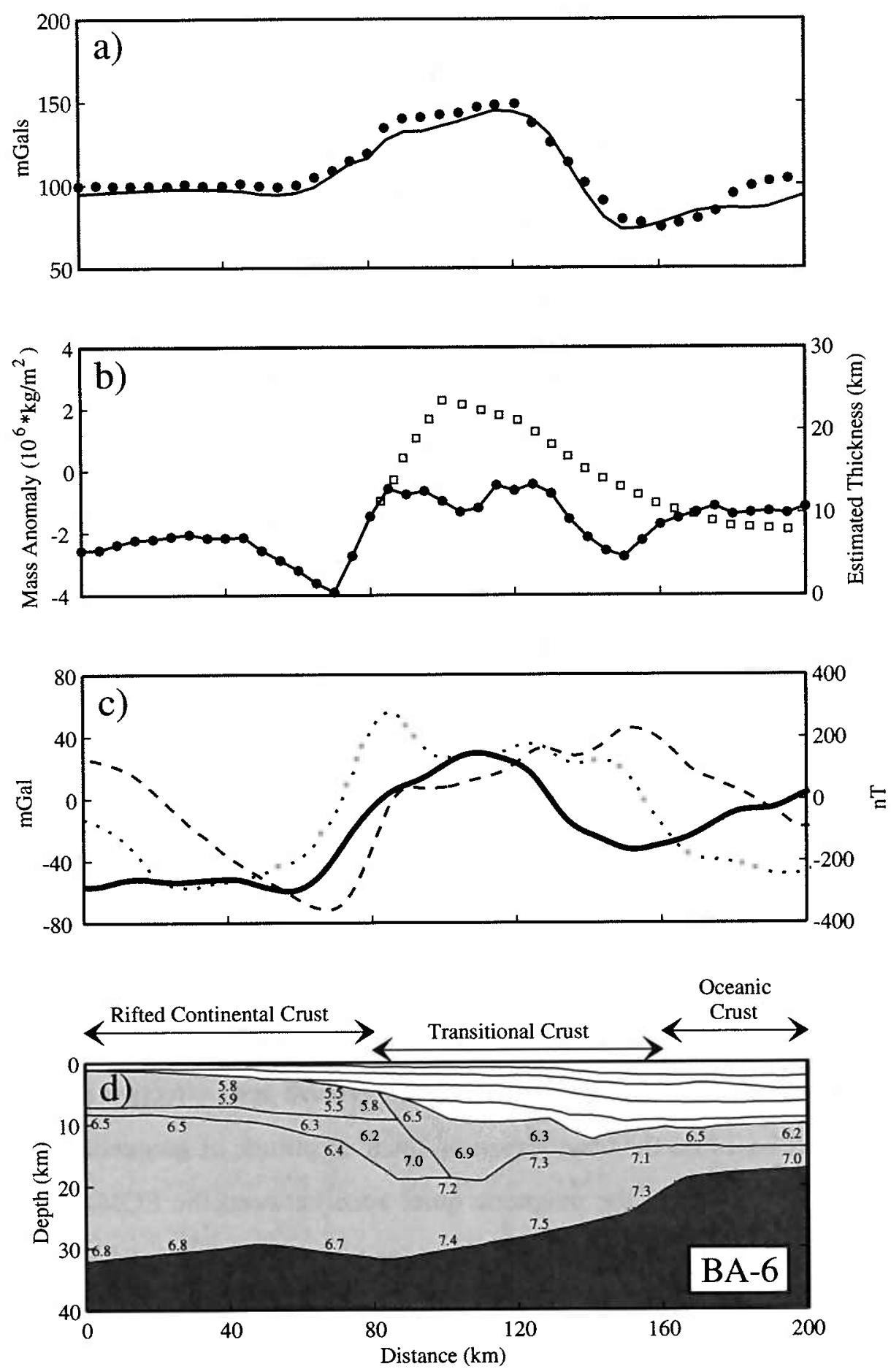

Figure 2-6: Comparison of the BA-6 seismic line [Holbrook et al., 1994b] to observed geophysical anomalies. See Figure 2-5 for notation. 

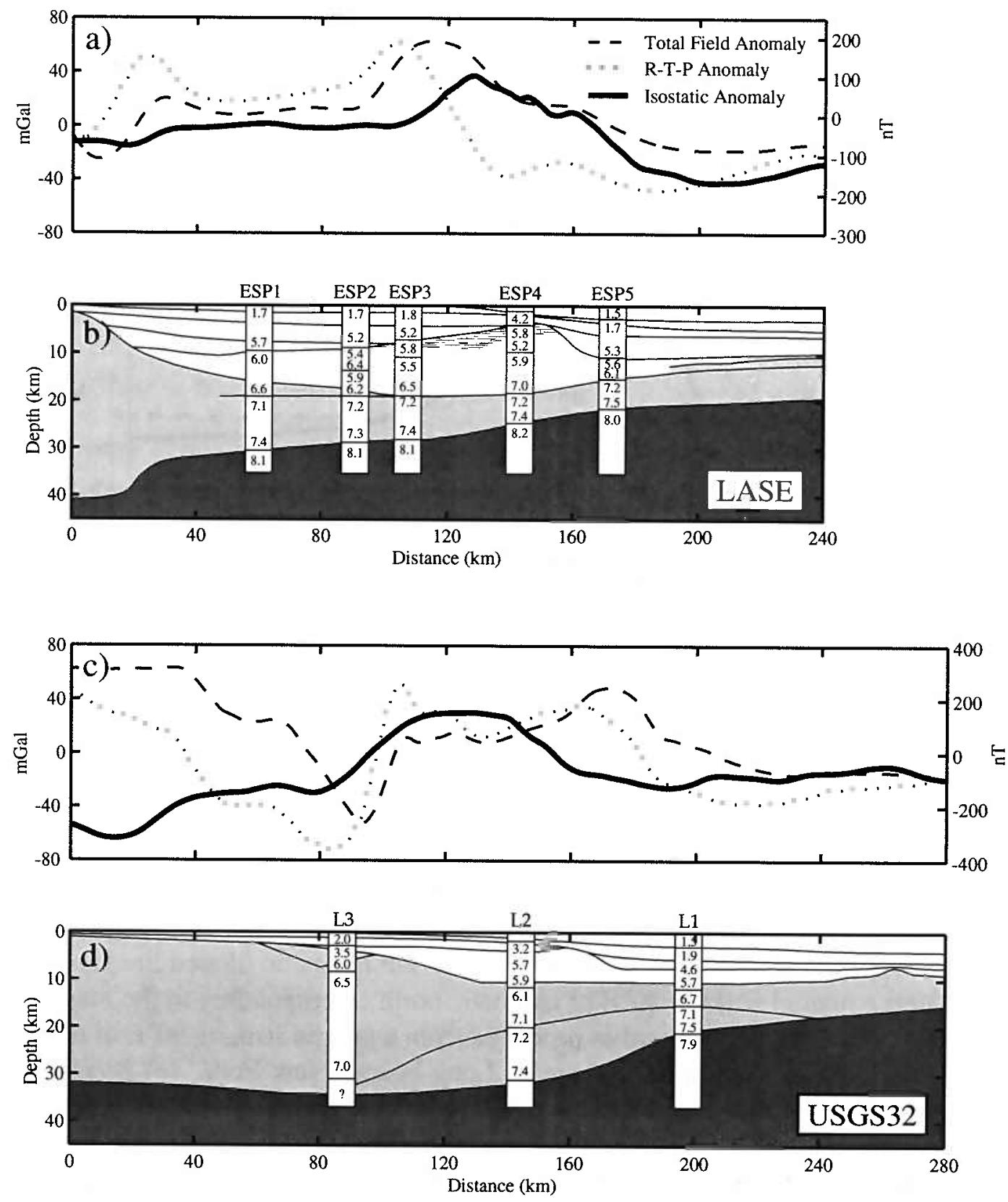

Figure 2-7: (a,b) Comparison of the LASE seismic line [LASE Study Group, 1986] to observed geophysical anomalies. Crustal velocities are constrained by five expanded spread profiles (ESPs) shown in Figure 2-7b. Note that along this profile the peak in the isostatic gravity anomaly lies seaward of the peak in the R-T-P anomaly and correlates closely with the location of the carbonate reef (patterned region) identified in the seismic profile. (c,d) Comparison of USGS32 seismic line [Klitgord et al., 1988] to observed geophysical anomalies. Crustal velocities are determined from three large-offset seismic profiles oriented perpendicular to the USGS32 line [Tréhu et al., 1989a] shown in Figure 2-7d. 

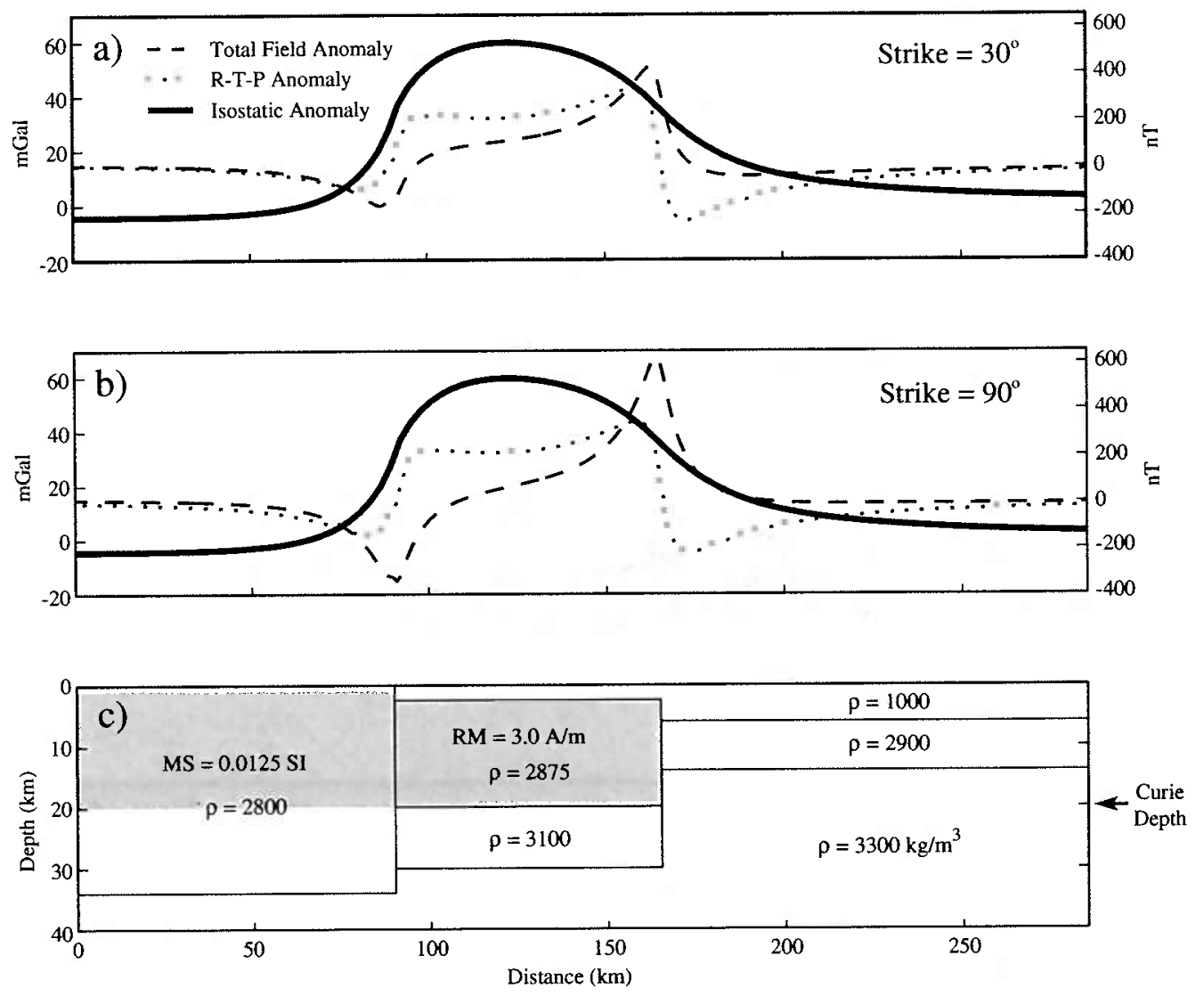

Figure 2-8: Illustration of gravity and magnetic anomalies produced by an idealized margin with underplated lower crust that is not in local isostatic equilibrium. (a) Isostatic gravity (solid line), total field magnetic (dashed line), and R-T-P magnetic (dotted line) anomalies produced from a margin striking $30^{\circ} \mathrm{E}$ of magnetic north corresponding to the margin offshore of North Carolina. (b) Anomalies produced from a margin striking $90^{\circ} \mathrm{E}$ of magnetic north corresponding to a location offshore of Long Island, New York. (c) Block model illustrating the densities and magnetizations used for the models shown in Figures 2-8a and $2-8 \mathrm{~b}$. Induced magnetization is assumed for the continental block with magnetic susceptibility (MS) of $0.0125 \mathrm{SI}$, while a remnant magnetization (RM) of $3.0 \mathrm{~A} / \mathrm{m}$ is used for the seaward dipping reflector sequence. The strength of the present-day magnetic field is taken to be $50,000 \mathrm{nT}$ with an inclination of $68^{\circ}$ and a declination of $-13^{\circ}$. We assumed an Early Jurassic paleofield for the remnant magnetization (inclination of $46^{\circ}$, declination of $-2.2^{\circ}$ [Austin et al., 1990]) and a Curie depth of $20 \mathrm{~km}$ for both models. The shift in the peak of the total field magnetic anomaly $\sim 50 \mathrm{~km}$ seaward of isostatic gravity high is similar to the observed seaward offset of the ECMA total field along the EDGE-801, BA-6, and USGS32 seismic lines. Density values of 2875 and $3100 \mathrm{~kg} / \mathrm{m}^{3}$ were assumed for the upper seaward dipping reflector block and lower high seismic velocity block, respectively. Densities were $2800 \mathrm{~kg} / \mathrm{m}^{3}$ for the continental crust block and $2900 \mathrm{~kg} / \mathrm{m}^{3}$ for the oceanic crust. 
sequence of high-density lower crustal rocks and corresponding seaward dipping reflectors in the upper crust associated with the formation of the East Coast margin.

In addition, it can be seen that the strike of the margin has a significant effect on the amplitude of the total field magnetic anomaly (Figure 2-8). We calculated two examples with strikes of $30^{\circ}$ and $90^{\circ}$ to represent the East Coast margin off North Carolina and Long Island, respectively. The calculated peak in the total field anomaly along the margin striking $90^{\circ}$ is $\sim 200 \mathrm{nT}$ greater than the margin striking $30^{\circ}$. Hence the observed increase in amplitude of the total field ECMA offshore of Long Island may represent the change in the margin trend and not necessarily an increase in the strength of the magnetic source body. Alternatively, the increase in amplitude of the total field ECMA toward the northeastern extreme of the margin may be caused by the observed shallowing of the basement depth in this region (Figure 2-9). Figure 2-9a illustrates that burying the idealized margin shown in Figure 2-8 with $5 \mathrm{~km}$ of nonmagnetized sediments decreases the peak of the total field magnetic anomaly by $\sim 50 \%$. We note that the isostatic gravity anomalies should not be affected by these variations in sediment thickness since the sediment effects have been taken into account during the gravity calculations.

To further investigate the relationship between the isostatic gravity anomaly and the seismic data, we used the seismically derived density models of Holbrook et al. [1994a, b] to calculate the isostatic mass anomaly along each profile (Figures 2-5 and 2-6). The mass anomalies were calculated by integrating the mass with depth in individual columns along each seismic profile and compared with the total mass of an idealized crustal reference model. Figures $2-5 b, 2-5 c, 2-6 b$, and $2-6 c$ show that there is a strong correlation between the isostatic mass anomaly as calculated from the density models of Holbrook et al. [1994a, b] and the isostatic gravity anomaly. To address the concern that the strong observed correlation may reflect the use of gravity data in the density modeling by Holbrook et al. [1994a, b], we also compared the isostatic gravity anomaly with the total estimated thickness of igneous rocks emplaced during rifting [Holbrook and Kelemen, 1993]. This method of analysis shows an excellent correlation between the isostatic gravity and the amount of material emplaced during rifting. Moreover, since the crustal thickness data 

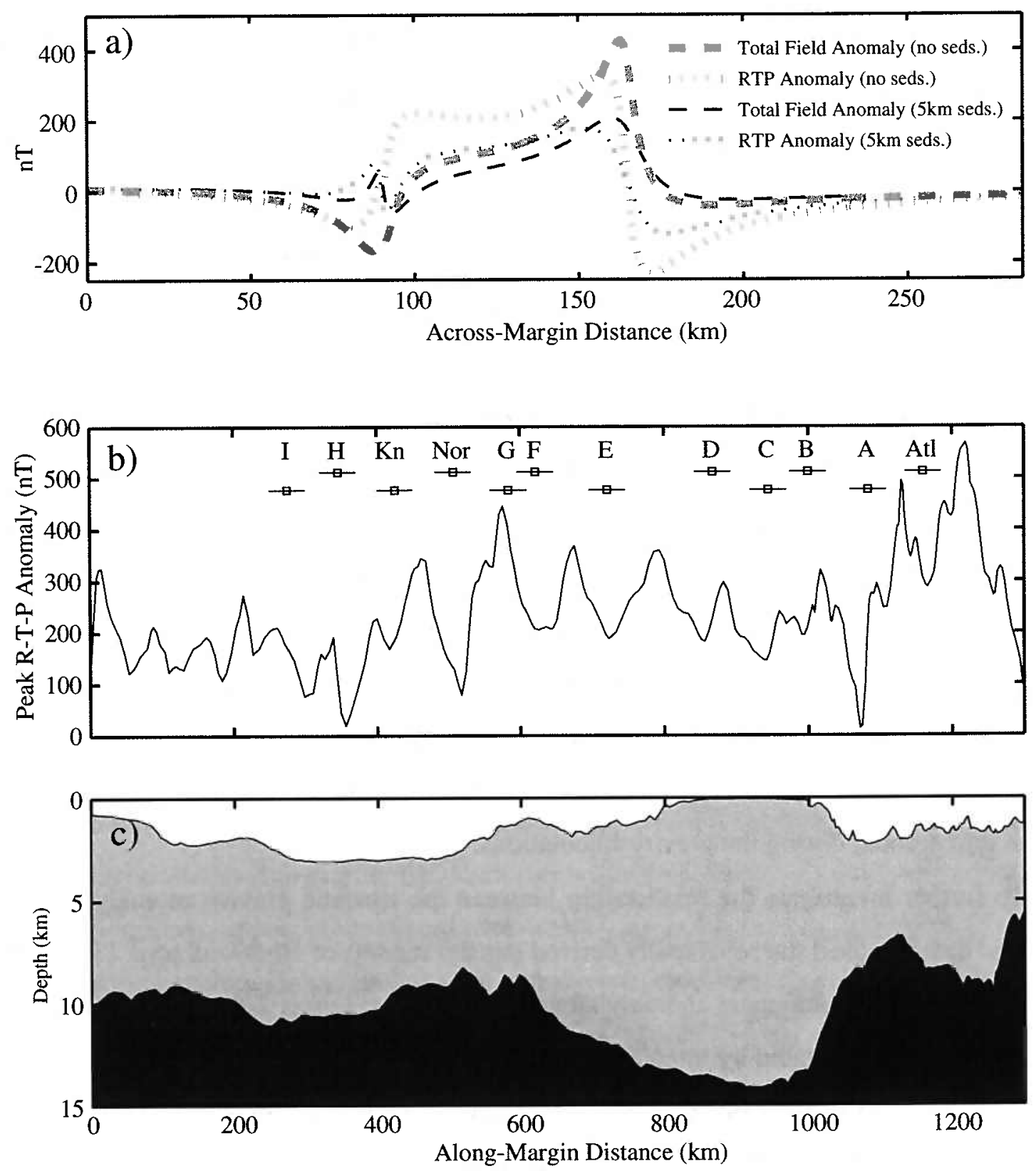

Figure 2-9: (a) Comparison of total field and R-T-P magnetic anomalies generated by a nonsedimented margin and a margin buried with $5 \mathrm{~km}$ of sediment. Model geometry is the same as in Figure 2-8c. (b) R-T-P magnetic anomaly along the peak of the ECMA. Alongmargin distance was calculated by projecting the profile in the average flow line direction and then taking the distance perpendicular to the flow lines as the standard distance. (c) Along-margin profile of bathymetry and basement depth (water, sediment, and crust shown in white, shaded, and black, respectively). Notice that the R-T-P anomalies are greatest toward the northeastern extreme of the margin where the basement depths are shallowest. 
have been interpreted directly from the seismic velocities, they contain no a priori dependence on the gravity signal. We conclude that the isostatic gravity anomaly is a useful indicator of variations in the amount of crustal volume along the margin.

On the basis of the cross-sectional area of the thickened igneous crust along the EDGE801 and BA-6 seismic lines (see Figures 2-5b and 2-6b, respectively), Holbrook and Kelemen [1993] estimated the total volume of igneous material emplaced along the East Coast margin to be $3.2 \times 10^{6} \mathrm{~km}^{3}$. However, Figure $2-4 \mathrm{c}$ shows that both the EDGE-801 and BA6 lines cross the margin at locations of relatively high values of isostatic gravity anomaly. Thus extrapolating the amount of igneous crust emplaced along the margin from these two seismic profiles alone may overestimate the total volume of igneous material. To provide a better estimation, we calculated a transfer function between the isostatic gravity anomaly and the thickness of igneous crust using the data from the EDGE-801 and BA- 6 seismic lines (Figure 2-10a). Clearly, density variations throughout the crustal column independent of underplating may also affect the isostatic anomaly, potentially leading to much of the scatter observed in Figure 2-10a. However, the correlations shown along the EDGE-801 and BA-6 lines in Figure 2-10a strongly suggest that the isostatic anomaly is largely dependent on the amount of emplaced igneous crust, and thus the isostatic anomaly can be used as a proxy for examining variations in the underplating process along the margin.

We applied the transfer function of Figure 2-10a to a series of across-margin profiles of isostatic gravity anomaly (spaced $1 \mathrm{~km}$ apart along the margin) to determine the crosssectional area of igneous material along each profile (Figure 2-10c). On the basis of the seismic profiles in Figures 2-5, 2-6, and 2-7 we estimated the maximum width of the magmatic emplacement zone to be $\sim 200 \mathrm{~km}$ and applied our transfer function to a $200 \mathrm{~km}$ wide window centered on the peak of the isostatic anomaly. In order to estimate only the excess igneous material emplaced during rifting, we subtracted from this value the amount of igneous material due to a normal thickness $(6 \mathrm{~km})$ oceanic crust seaward of the peak in the isostatic anomaly. Using this method, we calculated the amount of igneous material emplaced at individual across-margin profiles to range from 410 to $2300 \mathrm{~km}^{2}$, with an average value of $\sim 1400 \mathrm{~km}^{2}$ for the whole margin. This average value is $\sim 87.5 \%$ of the average 

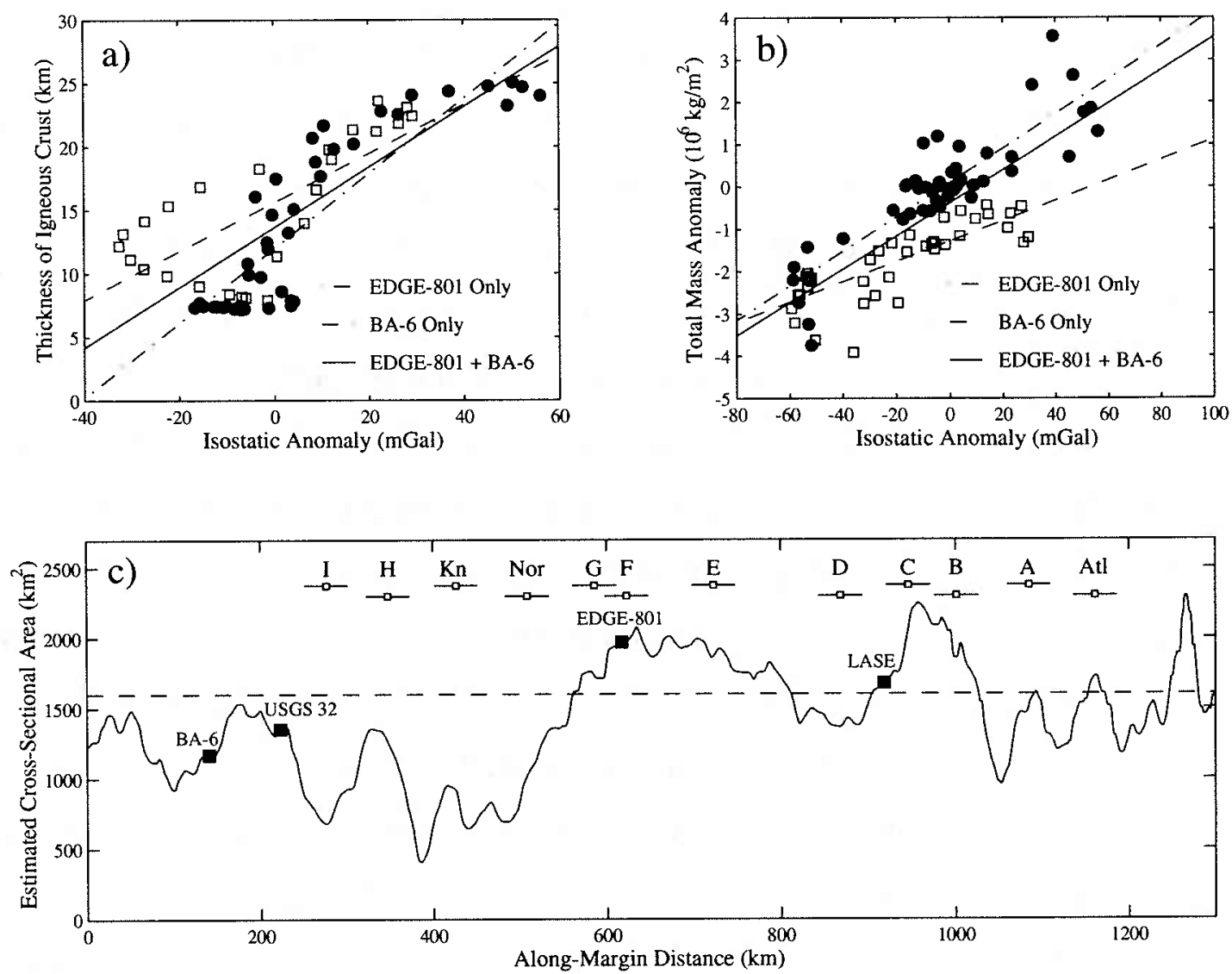

Figure 2-10: (a) Correlation between the isostatic gravity anomaly and the total thickness of igneous crust along the EDGE-801 (solid circles) and BA-6 (open squares) seismic lines. The transfer function calculated from the EDGE-801 data only (dash-dotted line) is $\mathrm{y}=$ $12.0+0.30 *$ isostatic anomaly $\left(\mathrm{r}^{2}=0.76\right)$; from the BA-6 data only (dashed line) is $\mathrm{y}=$ $15.6+0.19 *$ isostatic anomaly $\left(\mathrm{r}^{2}=0.53\right)$; and from both data sets combined (solid line) is $y=13.7+0.24 *$ isostatic anomaly $\left(\mathrm{r}^{2}=0.61\right)$. (b) Correlation between the isostatic gravity anomaly and the total mass anomaly along the EDGE- 801 (solid circles) and BA- 6 (open squares) seismic lines. The transfer function calculated from the EDGE-801 data only (dash-dotted line) is $\mathrm{y}=0.098+0.041 *$ isostatic anomaly $\left(\mathrm{r}^{2}=0.75\right)$; from the BA-6 data only (dashed line) is $y=-1.28+0.024 *$ isostatic anomaly $\left(\mathrm{r}^{2}=0.64\right)$; and from both data sets combined (solid line) is $\mathrm{y}=-0.38+0.039 *$ isostatic anomaly $\left(\mathrm{r}^{2}=\right.$ 0.66). (c) Along-margin variations in the estimated cross-sectional area of igneous material emplaced at individual across-margin profiles of isostatic gravity anomaly (spaced at $1 \mathrm{~km}$ apart along the margin). The estimation was based on the best fitting transfer function between the isostatic gravity anomaly and the total thickness of igneous crust using the combined data sets of the EDGE-801 and BA-6 seismic lines (solid line in Figure 2-10a). Dashed line illustrates the representative value of cross-sectional igneous material used by Holbrook and Kelemen [1993], which appears to overestimate the average values along the margin by $\sim 12.5 \%$. Solid squares show the locations of the four across-margin seismic lines identified in Figure 2-4. 
value from the EDGE-801 $\left(1800 \mathrm{~km}^{2}\right)$ and BA-6 $\left(1400 \mathrm{~km}^{2}\right)$ obtained by Holbrook and Kelemen [1993], suggesting that they may have overestimated the total amount of igneous material emplaced along the East Coast margin by $\sim 12.5 \%$. Our method assumes that the entire isostatic anomaly can be attributed to variations in the underplating process. However, in certain areas, density variations in the crustal column independent of underplating may also contribute to the isostatic anomaly (e.g., the carbonate reef observed in the LASE line). Thus our calculation of the amount of emplaced igneous material should be viewed only as a qualitative estimate for variations along the margin.

\subsubsection{Reduction-to-the-Pole Magnetic Anomalies}

Although isostatic gravity anomalies are typically situated directly over their source mass, this may not be the case for total field magnetic anomalies (Figure 2-8). Variations in magnetization direction and the direction of the Earth's present-day field may cause the total field anomaly to be distorted in shape and shifted laterally in position relative to its source mass. To correct for this effect, we performed a two-dimensional reduction-to-the-pole (R-T-P) analysis on the total field magnetic anomalies using the method of Blakely [1995]. The resulting R-T-P anomalies represent the magnetic anomaly that would be generated if both the magnetization and ambient field were directed vertically. Therefore anomalies that have been reduced to the pole are situated over their respective source body and altered in shape such that a symmetrical source generates a symmetrical anomaly.

In order to perform the reduction-to-the-pole calculation it is necessary to assume an inclination and declination for both the present-day field and the remanent magnetization direction. The mean inclination and declination of the present-day field across the East Coast margin were calculated from the IGRF to be $68^{\circ}$ and $-13^{\circ}$, respectively. The Jurassic field of Austin et al. [1990] (inclination of $46^{\circ}$, declination of $-2.2^{\circ}$ ) was assumed for the remanent magnetization direction. Figure 2-4d illustrates the results of the R-T-P analysis along the East Coast margin, showing that the peak of the ECMA has been shifted landward relative to the total field anomalies shown in Figure 2-3. Figures 2-5c and 2-6c illustrate 
this landward shift of the R-T-P anomaly along the EDGE-801 and BA-6 seismic lines, respectively. In both cases, the peak of the R-T-P anomaly aligns well with the sequence of seaward dipping reflectors identified from the seismic lines and the peak in the isostatic gravity anomaly calculated in this study.

The landward shift of the peak in the R-T-P anomaly relative to that of the total field magnetic anomaly is also observed in the simple forward block model shown in Figure 2-8. Our model illustrates the much more symmetrical shape of the R-T-P anomaly relative to the total field anomaly over the highly magnetized block representing the seaward dipping reflector sequence. Yet even in this simple case the R-T-P anomaly is not perfectly symmetric. This is due to the fact that when performing the reduction-to-the-pole calculation, it is necessary to assume a single magnetization vector. However, in our model the induced and remanent magnetization directions are slightly different. Thus even in this simple model it is impossible to remove all of the skewness in the total field magnetic anomaly. We stress this caveat when interpreting the R-T-P anomaly illustrated in Figure 2-4d. Nevertheless, we believe that because the current and Jurassic magnetic poles are relatively similar, reduction to the pole is an effective method for removing much of the skewness in the total field magnetic anomalies.

\subsubsection{Peaks in R-T-P Magnetic and Isostatic Gravity Anomalies}

The isostatic gravity anomaly shows a corridor of positive values running parallel to the East Coast margin (Figure 2-4c). Along most of the margin, the peak of the isostatic gravity anomaly is located $50-100 \mathrm{~km}$ landward of the peak of the total field magnetic anomaly but located close to the peak of the R-T-P anomaly. Figure 2-11 illustrates the spatial relationship between the peak of the isostatic gravity anomaly and the ECMA after the reduction-to-the-pole correction has been performed. To eliminate short-wavelength signals from the isostatic gravity anomaly, which are unlikely to reflect variations in deep crustal structure, we filtered the isostatic anomaly using a cosine cutoff taper to remove wavelengths $<50-100 \mathrm{~km}$. Along most of the margin the peaks of both fields are highly 


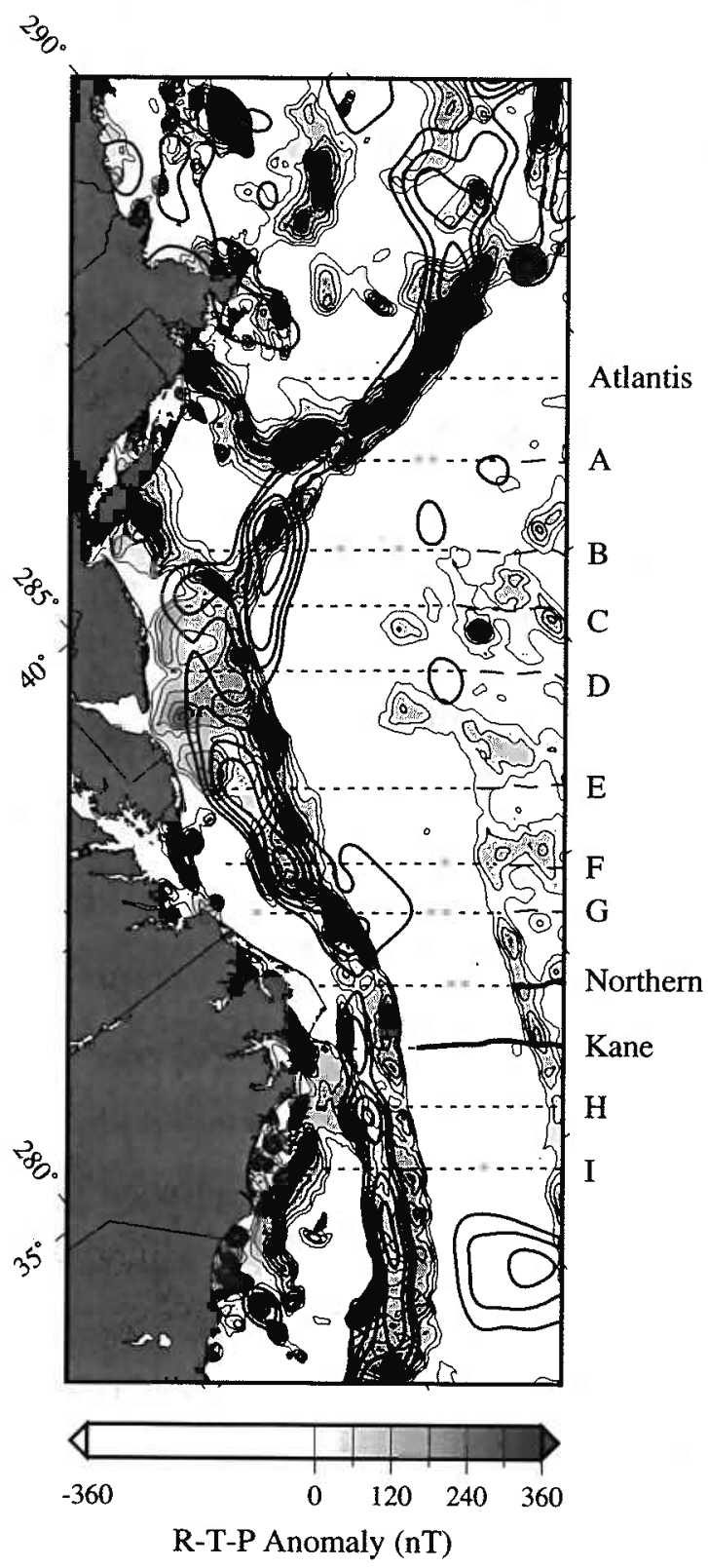

Figure 2-11: Comparison of R-T-P magnetic and isostatic gravity anomalies. Thin shaded contours illustrate R-T-P magnetic anomaly $>0 \mathrm{nT}$ with a contour interval of $60 \mathrm{nT}$. Thick contour lines without shading show filtered isostatic gravity anomaly greater than $0 \mathrm{mGal}$ with a contour interval of $10 \mathrm{mGal}$. Isostatic anomalies have been low-pass filtered using a cosine cutoff taper to remove wavelengths $<50-100 \mathrm{~km}$. Note the strong correlation between the peak of the R-T-P anomaly and the isostatic gravity high along much of the East Coast margin. Offset zone names and locations are the same as in Figure 2-3. 
correlated. Such a close spatial relationship between the peaks of the R-T-P magnetic and the isostatic gravity anomalies is consistent with a model in which highly magnetized extrusive rocks in the upper crust generate the observed peak in the R-T-P magnetic anomaly, while the corresponding peak in the isostatic gravity anomaly is caused by the integrated density effects of the high seismic velocity lower crust and the seaward dipping reflector sequence in the upper crust.

South of $35^{\circ} \mathrm{N}$ the isostatic gravity high appears to lie between a double peak in the R-T$\mathrm{P}$ anomaly. This double peak in the R-T-P anomaly may be caused by the falloff in magnetic anomaly toward the center of a magnetic source block, with the two peaks marking the horizontal extent of the magnetized block. This effect can be seen in Figure 2-8, where the R-T-P anomalies are calculated to be slightly higher at the edges of the magnetized block than at the center. The development of the double peak in the R-T-P anomaly should be more pronounced if the source body has sharp vertical boundaries, while bodies that taper toward the ends will produce a single R-T-P high located over the center of the magnetized body. (See Talwani et al. [1995] for the magnetic anomalies produced by a number of possible geometries for the seaward dipping reflector sequence.)

Between $35^{\circ} \mathrm{N}$ and the sharp change in trend of the margin around $39.5^{\circ} \mathrm{N}$, the peak in both the R-T-P and isostatic anomalies are wider and extend landward, potentially indicating a larger source body in this region. Another interesting feature of this section of the margin is that between $39^{\circ} \mathrm{N}$ and $39.5^{\circ} \mathrm{N}$ the isostatic gravity high is located seaward of the peak in the R-T-P anomaly. One potential explanation for this shift in the isostatic gravity high is the high density carbonate reef observed in the LASE seismic line, which lies directly below the peak in the isostatic gravity anomaly (Figures 2-7a and 2-7b). This carbonate reef might also explain the wider nature of the isostatic gravity anomaly in this area, with the high landward anomalies related to the underplated material and the high seaward anomalies related to the carbonate reef.

North of $39.5^{\circ} \mathrm{N}$ the peak of the two fields align closely again. The magnitude of the R-T-P anomaly in this region is significantly greater than elsewhere along the margin; however, unlike the sections of the margin farther south, no double peak is observed here. 
In contrast, the isostatic gravity anomaly north of $39.5^{\circ} \mathrm{N}$ is subdued relative to the isostatic anomalies farther south. One possible interpretation of this contrast in the strength of the RT-P and isostatic anomalies is that while the volume and width of the underplated material may be smaller north of $39.5^{\circ} \mathrm{N}$, the R-T-P anomaly remains high due to the shallowing of the basement. This explanation would also account for both the small isostatic anomalies and the lack of a double peak in the R-T-P anomaly due to a decrease in width of the seaward dipping reflector sequence.

\subsubsection{Conjugate West African Margin}

In order to better understand the implications of the segmentation pattern observed along the U.S. East Coast margin, we also calculated isostatic gravity anomalies for the conjugate West African margin. Because sediment thickness data are not available for this region, the isostatic gravity anomaly was calculated by simply subtracting from free-air gravity the effects of a water-crust $\left(\Delta \rho=1700 \mathrm{~kg} / \mathrm{m}^{3}\right)$ and crust-mantle $\left(\Delta \rho=600 \mathrm{~kg} / \mathrm{m}^{3}\right)$ interface. The free-air gravity for the conjugate margin was extracted from the Sandwell and Smith [1997] satellite gravity map and the bathymetry was taken from ETOPO5. The depth to the crust-mantle interface was calculated from the ETOPO5 bathymetry assuming local Airy isostasy. The residual isostatic gravity anomaly for the West African margin is shown in Figure 2-12a. Similar to the isostatic anomaly shown in Figure 2-11, we have filtered the anomaly along the West African margin using a cosine cutoff taper to remove wavelengths $<50-100 \mathrm{~km}$. Note the strong similarity between the pattern of isostatic anomaly along the West African margin and that along the U.S. East Coast.

\subsection{Along-Margin Segmentation and Implications}

Profiles of the peak in the R-T-P magnetic and isostatic gravity anomalies along the U.S. East Coast margin show both anomalies to be strongly segmented at various wavelengths (Figures 2-13a and 2-13c). To facilitate discussion in this paper, we call anomalies with 

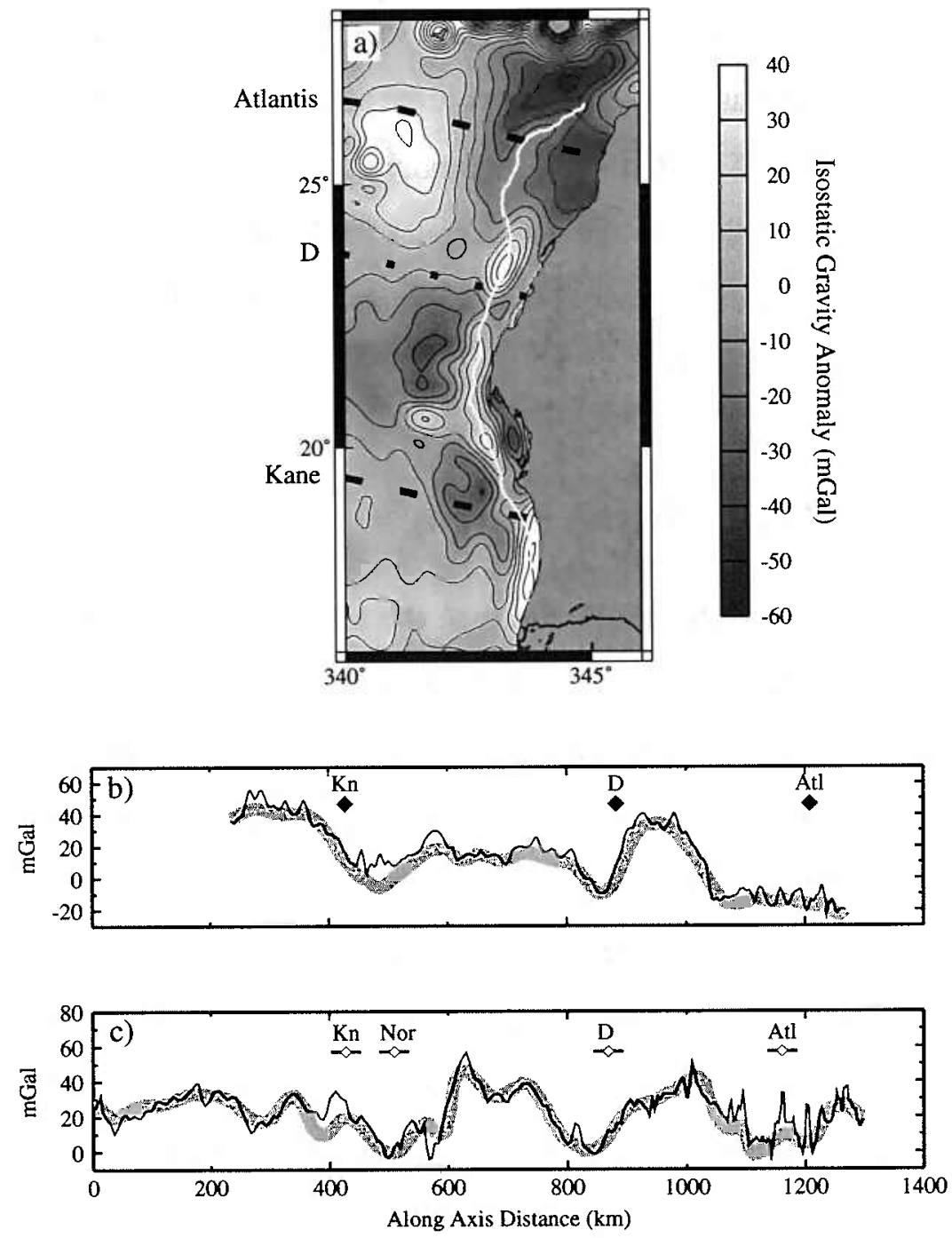

Figure 2-12: (a) Isostatic gravity anomaly along the West African margin conjugate to the U.S. East Coast margin, calculated by subtracting from the free-air gravity the effects of the water-crust $\left(\Delta \rho=1700 \mathrm{~kg} / \mathrm{m}^{3}\right)$ and crust-mantle $\left(\Delta \rho=600 \mathrm{~kg} / \mathrm{m}^{3}\right)$ interfaces. Isostatic anomalies have been low-pass filtered using a cosine cutoff taper to remove wavelengths $<50-100 \mathrm{~km}$. Dashed lines show the location of the Kane and Atlantis fracture zones projected from the Mid-Atlantic Ridge using the flow lines of Klitgord and Schouten [1986]; dotted line represents location of the D offset zone. Thin white line illustrates the location of profile in Figure 2-12b. (b) Variations in the peak of the isostatic gravity anomaly along the West African margin. Locations of Kane (Kn), Atlantis (Atl), and D offset zones are marked with solid diamonds (no estimates of uncertainty were made). (c) Variations in the peak of the isostatic gravity anomaly along the U.S. East Coast margin. Notice the similar $300-500 \mathrm{~km}$ wavelength variations in the isostatic gravity anomalies along both margins, with the Kane, Atlantis, Northern (Nor), and D offset zones located in the isostatic gravity lows. 
spatial wavelengths $<50 \mathrm{~km}$ very short-wavelength features, those between 50 and $250 \mathrm{~km}$ short-wavelength features, and those between 250 and $500 \mathrm{~km}$ intermediate-wavelength features. Spectral analyses of the along-margin profiles reveal that the isostatic gravity anomaly has two distinct spectral peaks (Figure 2-13d) at relatively short (100-150 km) and intermediate wavelengths $(300-500 \mathrm{~km})$, while most of the signal power in the R-T-P ECMA corresponds to only the short wavelengths (100-120 km) (Figure 2-13b). Since the GSC magnetic field data were filtered to remove wavelengths $>400 \mathrm{~km}$ [Verhoef et al., 1996], the lack of a spectral peak at the intermediate wavelengths in the R-T-P magnetic anomaly may be partially an artifact of the initial data processing. The short-wavelength segmentation in the isostatic gravity anomaly is of smaller amplitude (15-30 mGal) than the intermediate-wavelength segmentation (40-60 mGal).

\subsubsection{Comparison to the Present-Day MAR Axis Segmentation}

Crustal magnetization [Sempéré et al., 1993; Pockalny et al., 1995] and mantle Bouguer anomalies (MBA) [Escartín and Lin, 1998; Thibaud et al., 1998] are also observed to vary along the present-day MAR axis (Figures 2-13e and 2-13g). Similar to the power spectra from the along-margin R-T-P magnetic and isostatic gravity anomalies (Figures 2-13b and 2-13d), the crustal magnetization of the MAR axis shows a spectral peak around a wavelength of 100-120 km (Figure 2-13f). The MBA of the MAR axis also shows a shortwavelength spectral peak (Figure $2-13 \mathrm{~h}$ ) but at a slightly longer wavelength $(\sim 150 \mathrm{~km})$. The reason for the difference between the short-wavelength peak in the MBA and other three anomalies is unclear. However, it is possible that smaller offsets at the modern MAR do not provide enough variation in crustal thickness to produce a significant MBA low, thus biasing the spectral peak in the MBA toward longer wavelengths.

The along-axis magnetization and MBA show an additional spectral peak at very short wavelength of around $40-50 \mathrm{~km}$, corresponding to the observed $20-80 \mathrm{~km}$ along-axis length of individual spreading segments bounded primarily by nontransform offsets at the MAR axis between the Kane and Atlantis fracture zones [Sempéré et al., 1993]. Notice 

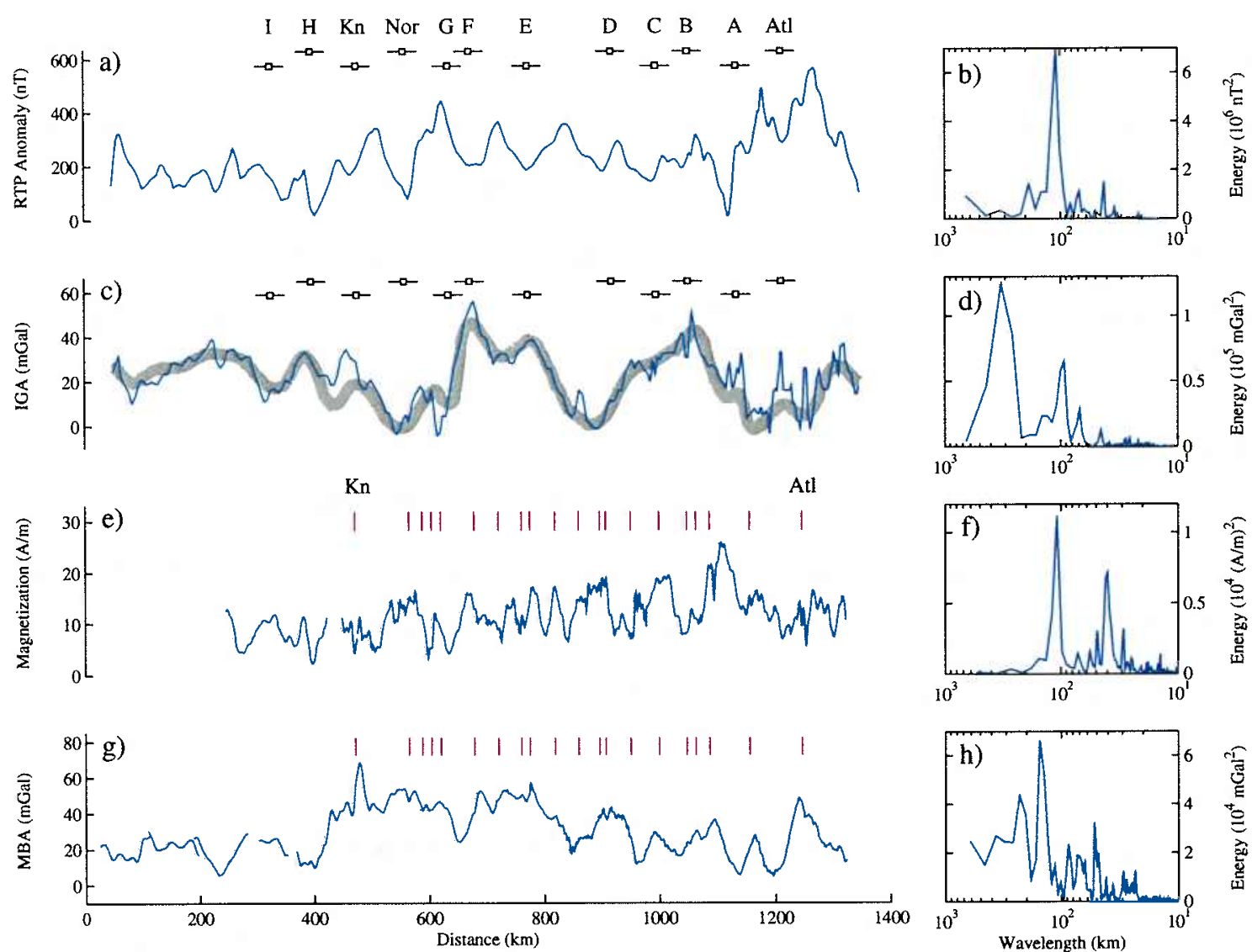

Figure 2-13: Comparison of variations in R-T-P magnetic and isostatic gravity anomalies along the East Coast margin to along-axis changes in crustal magnetization and mantle Bouguer anomaly (MBA) at the present-day Mid-Atlantic ridge (MAR). (a) R-T-P magnetic anomaly along the peak of the ECMA. Open squares represent the locations where the offset zones enter the margin, with bars illustrating $\pm 25 \mathrm{~km}$ zones of estimated uncertainty. Along-margin distance was calculated by projecting the profile in the average flow line direction and then taking the distance perpendicular to the flow lines as the standard distance. (b) Variance conserving power spectrum for the alongmargin R-T-P magnetic anomaly. (c,d) Along-margin variations in the peak of the isostatic gravity anomaly with corresponding power spectrum. The thick shaded line illustrates the filtered isostatic gravity anomaly using a cosine cutoff taper to remove wavelengths $<50-100 \mathrm{~km}$. (e,f) Along-axis crustal magnetization at the present-day MAR and corresponding power spectrum. Thin vertical lines represent the location of Kane and Atlantis transform faults (Kn and Atl) and numerous nontransform offsets [Escartín and Lin, 1998]. Data south of the Kane fracture zone are taken from Pockalny et al. [1995], while the data north of Kane are from Sempéré et al. [1993]. The crustal magnetization was calculated by downward continuation of residual magnetic anomalies assuming a 0.5-km thick magnetic source layer in the upper crust [Sempéré et al., 1993; Pockalny et al., 1995]. $(\mathrm{g}, \mathrm{h})$ Along-axis MBA and corresponding power spectrum. Data south of the Kane fracture zone are taken from Thibaud et al. [1998], while data north of Kane are from Escartín and Lin [1998]. Note that all four data sets show a characteristic segmentation wavelength of $100-150 \mathrm{~km}$. However, only the along-margin isostatic gravity anomaly and the along-axis MBA gravity show significant spectral power at wavelengths $>200 \mathrm{~km}$. 
that the peak in the MBA is at a slightly longer wavelength $(\sim 50 \mathrm{~km})$ than the spectral peak in the crustal magnetization $(\sim 40 \mathrm{~km})$. This discrepancy may be caused by the fact that the MBA is generated by variations in Moho depth at the base of the oceanic crust, while the variations in magnetization are generated within the extrusive layer in the top 1 $\mathrm{km}$ of the crust. The lack of this very short-wavelength spectral peak in the along-margin R-T-P magnetic and isostatic gravity anomalies may be related to the fact that the margin potential field data are highly attenuated from the source crust, typically buried beneath 5$12 \mathrm{~km}$ of sediments. Similar to the along-margin R-T-P magnetic anomaly, no significant power is seen in the crustal magnetization along the present-day MAR axis at wavelengths $>200 \mathrm{~km}$. However, the along-axis MBA profile does show signal power at the intermediate wavelengths, although it does not develop a distinct spectral peak between 300 and 500 $\mathrm{km}$ as in the along-margin profile of the isostatic gravity anomaly.

\subsubsection{Relation to the Incipient Atlantic Offset Zones}

The Kane and Atlantis fracture zones are the largest and most stable offsets along the section of the present-day MAR that corresponds to the East Coast margin (Figure 2-1). The traces of the Kane and Atlantis fracture zones intersect both the U.S. East Coast margin and the conjugate West African margin near lows in the intermediate-wavelength (300-500 $\mathrm{km}$ ) isostatic gravity anomaly (Figures 2-12 and 2-13), although at both margins the Northern offset zone is located slightly closer to the southern isostatic gravity low than the Kane fracture zone. No other major fracture zone is observed at the present-day MAR between Kane and Atlantis (Figure 2-1), as would be predicted from the additional intermediatewavelength gravity low located near offset zone D (see Figures 2-11 and 2-12).

Correlating the short-wavelength $(100-150 \mathrm{~km})$ segmentation in the R-T-P magnetic and isostatic gravity anomalies with the incipient locations of the hypothesized offset zones along the U.S. East Coast is much more difficult to accomplish because the spatial uncertainties in the offset zone identification are almost as large as the spatial scales of the shortwavelength along-margin anomalies. Nevertheless, several of the incipient offset zones 
(e.g., Atlantis, A, C, D, G, Northern, Kane, H, and I) appear to correlate with lows in both the R-T-P magnetic and isostatic gravity anomalies at the short wavelengths $(100-150 \mathrm{~km})$ (Figure 2-11).

\subsubsection{Correlation With Across-Margin Isostatic Gravity Anomalies}

Along-margin segmentation in the isostatic gravity anomaly at the intermediate wavelengths $(300-500 \mathrm{~km})$ also appears to be associated with the across-margin variations in the isostatic gravity anomaly. Figure $2-14$ a shows 20 across-margin profiles taken at the individual short-wavelength $(100-150 \mathrm{~km})$ peaks and troughs of the isostatic gravity anomaly along the margin (see Figure $2-14 \mathrm{~b}$ for profile locations). In regions where the intermediate-wavelength $(300-500 \mathrm{~km})$ along-margin isostatic anomaly is generally high, the across-margin profiles are often characterized by a prominent peak and an adjacent low in the anomaly (i.e., in profiles $1-4,8-10,12-16,18-20$ ). This occurs regardless of whether the across-axis profile is taken at a short-wavelength $(100-150 \mathrm{~km})$ peak or trough in the along-margin anomaly. Conversely, in regions located within lows of the intermediate-wavelength $(300-500 \mathrm{~km})$ isostatic gravity anomaly, the across-margin profiles are relatively flat without a significant high-low couple (i.e., in profiles 5-7, 11, and 17). This relationship is further illustrated in Figure 2-14c, which shows a positive correlation between the peak value of the across-margin isostatic gravity anomaly and the mean across-margin gradients. The mean gradients were calculated by averaging the absolute value of point-to-point gradients in a $200 \mathrm{~km}$ spatial window around the peak value of the isostatic gravity anomaly. This relationship is significant because it shows that the isostatic gravity anomaly has a relatively uniform base value of $0 \mathrm{mGal}$, but in certain locations varies above this value, potentially reflecting enhanced underplating in these regions. We again note that profiles 5-7 are located near the incipient location of the Kane FZ, profile 17 is near the incipient location of the Atlantis FZ, and profile 11 is near the incipient location of the D offset zone. 

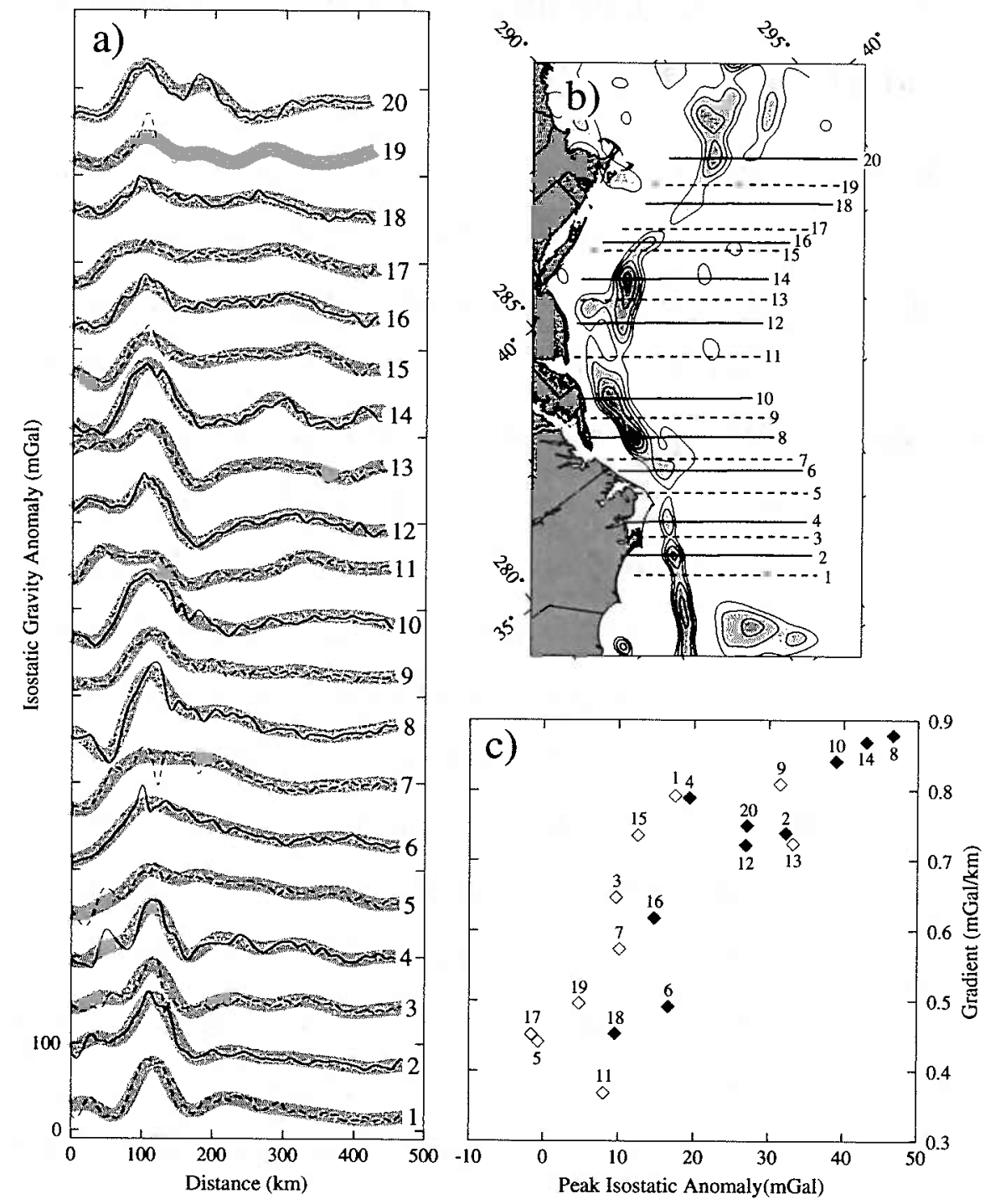

Figure 2-14: (a) Across-margin profiles showing variations in the isostatic gravity anomaly. Solid lines represent profiles taken through $100-150 \mathrm{~km}$ wavelength peaks in the isostatic gravity anomaly, while dashed lines show profiles taken through 100-150 km wavelength isostatic lows. Thick shaded lines represent the filtered isostatic anomaly calculated using a cosine cutoff taper to remove wavelengths $<50-100 \mathrm{~km}$. (b) Location of profiles shown in Figure 2-14a. Shaded contours illustrate filtered isostatic gravity anomalies greater than $0 \mathrm{mGal}$ with a contour interval of $10 \mathrm{mGal}$. (c) Mean absolute gradient in across-margin anomaly versus the peak value of the isostatic gravity anomaly. The mean absolute gradient was calculated along an across-margin profile in a $200 \mathrm{~km}$ window around the peak value of the isostatic anomaly. Solid symbols represent across-margin profiles taken through 100 $150 \mathrm{~km}$ wavelength isostatic highs, while open symbols represent across-margin profiles taken through isostatic lows. Note the positive relationship between the mean absolute across-margin gradient and the peak of the isostatic anomaly, indicating that as the peak of the isostatic anomaly decreases, the overall across-margin variation becomes smaller. 


\subsubsection{Kane and Atlantis Fracture Zones as Boundaries of Tectonic Corridors?}

The $300-500 \mathrm{~km}$ segmentation in the along-margin isostatic gravity anomaly is similar in wavelength to the tectonic corridors of the lithosphere seen by Kane and Hayes [1992] in the South Atlantic. Kane and Hayes [1992] observed tectonic corridors persisting for tens of millions of years, which exhibit large, along-ridge-axis variations in subsidence rate, zero-age seafloor depth, geoid rate as measured by geoid height decrease with age, and geochemical anomalies on length scales of $500-1000 \mathrm{~km}$. These tectonic corridors are often bounded by major fracture zones. It has been suggested that these corridors are caused by changes in mantle temperature along and across the axis of a mid-ocean ridge and may have been present from the time of initial rifting and ridge formation [Kane and Hayes, 1994]. Such a deep mantle anomaly is not expected to have a direct effect on crustal magnetization, which has its source at depths above the Curie temperature, but would be expected to produce significant variations in isostatic gravity along the margin.

Our new observation of the clear correlation of the Kane and the Atlantis FZs with the intermediate-wavelength $(300-500 \mathrm{~km})$ isostatic gravity lows supports a hypothesis that the Kane and Atlantis FZs might be the boundaries of a tectonic corridor in the North Atlantic, similar to those observed by Kane and Hayes $[1992,1994]$ in the South Atlantic. One important implication of the tectonic corridor concept is that it predicts the boundaries of the corridor (such as the Kane and Atlantis FZs) to be much more stable and prominent in comparison to offsets occurring within the corridor interior (such as the numerous nontransform offsets observed along the present-day MAR axis between the Kane and Atlantis FZs). Another implication is that the intermediate-wavelength isostatic gravity anomaly might be a good indicator of tectonic corridors globally. These hypotheses can be tested through future investigations of other continental margins using multiple investigative approaches including the isostatic gravity analysis. 
The direct cause of the observed isostatic gravity anomalies along the U.S. East Coast margin may be a combination of along-margin variations in both the amount of thickened igneous crust and the strength of the lithosphere. If igneous crustal accretion along a volcanic margin is focused toward discrete centers similar to that of an active slow spreading center, we would expect crustal underplating to be least near offset zones and greatest at the segment centers of a margin. Furthermore, it is possible that the intermediate-wavelength variations in isostatic gravity anomalies reflect the characteristic size of mantle upwelling cells during continental rifting.

In addition, we hypothesize that at incipient oceanic offset zones, especially near the boundaries of tectonic corridors, the oceanic lithosphere is relatively weak and thus incapable of supporting large tectonic loads. This results in smaller values of along- and across-margin isostatic gravity anomalies, as observed near the incipient locations of the Kane and Atlantis fracture zones. Sawyer [1985] and Dunbar and Sawyer [1989] showed that preexisting weaknesses in the structure of the U.S. East Coast may have controlled the early stages of extension along the margin. These studies concluded that seafloor spreading will tend to be focused in preexisting weak zones, leading to smaller amounts of extension in these areas relative to regions of higher initial strength. The Kane, Atlantis, and D offset zones all fall into regions of low total extension as identified by Dunbar and Sawyer [1989] and thus can be hypothesized to represent initial weaknesses in the U.S East Coast margin. Together with the possible variable underplating mechanism, such changes in lithospheric strength could lead to even greater along- and across-margin variations in isostatic gravity anomalies.

Changes in lithospheric strength have also been observed along other rifted continental margins. For example, by analyzing changes in the structure of the continental edge effect, Watts and Marr [1995] found lithospheric strength to vary on length scales of several hundred kilometers along the African margin. Watts and Marr [1995] correlated these variations to the location of hot spot traces, suggesting that the incidence of a hot spot on the base of the lithosphere may be capable of decreasing its effective elastic thickness. More recent work by Watts and Stewart [1998] found a 350-400 km weak zone to exist along the 
Gabon margin offshore of West Africa, suggesting that similar scale strong versus weak segmentation may be a characteristic of many rifted continental margins.

At present, however, the relative importance of the variable underplating versus variable lithospheric strength is poorly known. Recent seismic experiments along active spreading centers have played a key role in determining the relative importance of the variations in crustal thickness/density versus upper mantle structure in producing the "bull's-eye" MBA patterns observed along the MAR [Tolstoy et al., 1993; Canales et al., 2000b; Hooft et al., 2000]. To date, most high-quality seismic experiments at volcanic margins have been carried out only in across-margin lines. The results of this study suggest a strong need for well-designed future seismic experiments to be conducted along the margins.

\subsection{Conclusions}

In this study we examine variations in the magnetic field and the isostatic gravity anomalies along the U.S. East Coast margin. We observe a corridor of high isostatic gravity anomaly running along the margin that corresponds closely to the location of the ECMA after reduction to the pole of the total field magnetic anomaly. Both of these anomalies situate close to the maximum thickness of high velocity igneous crust determined by previous seismic studies. Spectral analysis shows the R-T-P ECMA is segmented on length scales of 100 $120 \mathrm{~km}$, while the isostatic gravity anomaly is found to exhibit segmentation in two distinct wave bands: $100-150 \mathrm{~km}$ and $300-500 \mathrm{~km}$. We find the $300-500 \mathrm{~km}$ segmentation in the isostatic gravity anomaly to have a larger amplitude $(40-60 \mathrm{mGal})$ than the $100-150 \mathrm{~km}$ wavelength (15-30 mGal) and to coincide with an overall change in the character of the across-margin isostatic gravity anomalies.

The observed segmentation in gravity and magnetic anomalies at the U.S. East Coast margin has important implications for the formation of continental margins and the origin of segmentation at mature mid-ocean ridges. The short-wavelength $(100-150 \mathrm{~km})$ alongmargin magnetic and isostatic gravity anomalies are similar in wavelength to the segmentation in magnetization and mantle Bouguer anomaly observed along the present-day MAR 
axis. Moreover, many of the short-wavelength magnetic and isostatic gravity lows correlate well to the location of early traces of identifiable Atlantic offsets. While this correlation is not one to one, such a relationship would not necessarily be expected due to the along-axis migration of nontransform offsets with time and the thick sedimentary basins attenuating the crustal gravity signal near the margin.

The $300-500 \mathrm{~km}$ segmentation in the along-margin isostatic gravity anomaly is similar in scale to the intermediate-wavelength tectonic segmentation observed in the South Atlantic [Kane and Hayes, 1992], for which a mantle source has been proposed. The intermediate-wavelength $(300-500 \mathrm{~km})$ isostatic gravity lows correspond to the early traces of the Kane and Atlantis FZs, suggesting that these two fracture zones may define a single tectonic corridor in the North Atlantic. The intermediate-wavelength segmentation is also similar to variations in lithospheric strength observed along the African margin [Watts and Marr, 1995; Watts and Stewart, 1998]. We hypothesize that the direct cause of the intermediate-wavelength isostatic gravity anomaly is along-margin variations in both the amount of the underplated igneous crust and the strength of the lithosphere, although the relative importance of these two effects remains unresolved. Our results imply that segmentation is an important feature of margin development and that segmentation at mature oceanic spreading centers may be directly linked to segmentation during continental rifting. 


\title{
Chapter 3
}

\section{A continuum mechanics model for}

\section{normal faulting using a strain-rate}

\section{softening rheology*}

\begin{abstract}
We use a strain-rate dependent visco-plastic finite element model to investigate the effects of crustal rheology and thermal structure on the development of normal faults in 2-D extending lithosphere. Strain-rate softening in the brittle regime is used to simulate the rate-dependence of frictional strength observed in laboratory studies. Results of numerical experiments show that efficient strain-rate softening can result in localized zones of high strain rate, which develop in response to the rheology and boundary conditions and are not imposed a priori. We argue that these zones of localized shearing are analogous to faults and use the calculated deformation field to predict the preferred location of fault formation for a range of thermal and rheologic conditions. When no regional temperature gradient is imposed, deformation is predicted to be distributed between several sets of conjugate normal faults. However, in the presence of a horizontally varying temperature field, faulting is calculated to focus where the lithosphere is thinnest, forming rift-like topography. Model results predict that when crustal thickness is sufficiently small that no ductile layer forms in the lower crust, deformation is mantle-dominated and rift half-width is controlled primarily by the geothermal gradient. In contrast, when crustal thickness is large enough that the stress accumulation in the upper crust becomes much greater than the stress accu-
\end{abstract}

\footnotetext{
*In revision: M.D. Behn, J. Lin, and M.T. Zuber, A continuum mechanics model for normal faulting using a strain-rate softening rheology: Implications for thermal and rheological controls on continental and oceanic rifting, Earth Planet. Sci. Lett., 2002
} 
mulation in the upper mantle, deformation becomes crust-dominated and the calculated rift half-width is a function of both crustal rheology and the geothermal gradient. This implies that under typical geological conditions, the brittle upper crust does not deform independently of a strong upper mantle. Therefore, complete decoupling of the upper crust from the upper mantle may not occur in many extensional systems.

\subsection{Introduction}

Rift zones are areas of localized lithospheric extension, typically characterized by a central basin bounded on either side by inward dipping normal faults. Rift flanks are generally uplifted, and the lithosphere beneath a rift zone is often abnormally thin. Extensional environments are commonly associated with regional uplift, high heat flow, and local magmatism, indicating the presence of a thermal anomaly. Rift zones are observed in both continental (e.g., Rio Grande Rift, Rhine Graben) and oceanic lithosphere (e.g., mid-ocean ridges), and represent an important mechanism for continental breakup and seafloor spreading. In this study, we investigate the relationship between the development of rift systems and the rheologic properties of the lithosphere.

The rheologic structure of the lithosphere is often assumed to consist of a brittle layer overlying a ductile region at depth [Brace and Kohlstedt, 1980; Kohlstedt et al., 1995]. In the brittle regime, strain is accomodated on faults when stress exceeds the frictional resistance on a fault plane. Friction on rock surfaces has been shown to be a complex non-linear function of overburden pressure [Byerlee, 1978], strain and loading history [Dieterich, 1979; Ruina, 1983], and sliding velocity [Rabinowicz, 1965; Dieterich, 1972]. In the ductile regime, strain is controlled by a temperature-dependent power-law that relates stress and strain-rate [Kirby, 1983]. Using these relationships, experimentally derived friction and flow laws for crustal and mantle rocks can be incorporated into numerical models of rifting in order to improve our understanding of the tectonic processes that create rift morphology.

A common formulation for the development of normal faults in extensional environments is to treat the lithosphere as an elastic layer floating on an inviscid fluid [e.g., Ven- 
ing Meinesz, 1950; Bott, 1981; Forsyth, 1992; Buck, 1993; Shaw and Lin, 1993, 1996; Lavier et al., 2000]. Normal faults are assumed to penetrate the lithosphere, with displacement on an individual fault accumulating until the shear stresses exceed the brittle strength of the lithosphere and a new fault is formed. Unfortunately, these models generally require that the location and dip of faults be specified a priori [e.g., Melosh and Raefsky, 1981; Melosh and Williams, 1989; Boutilier and Keen, 1994; Chéry, 2001], rather than developing in response to the evolving stress field. An alternative class of models assumes that the lithosphere behaves as a non-Newtonian viscous fluid. This approach has been used to describe rift morphology [Zuber and Parmentier, 1986; Buck, 1991], the characteristic spacing of faults [Fletcher and Hallet, 1983; Zuber et al., 1986], and dynamic topography [Chen and Morgan, 1990] in extensional environments. An assumption of these continuum models is that deformation occurs on closely spaced faults that can be approximated by plastic flow.

Recently, several workers have begun to incorporate the interaction between non-linear frictional resistance laws and non-Newtonian viscous flow laws in continuum models of lithospheric extension. Poliakov and Buck [1998] simulated cohesion loss on a fault surface by including strain softening in models of abyssal hill formation at mid-ocean ridges. Similarly, Lavier et al. [2000] used cohesion loss to model the development of low angle normal faults in continental environments. Using a different approach, Frederiksen and Braun [2001] illustrated that strain softening in the ductile regime could lead to strain localization and the development of mantle shear zones during lithospheric extension. However, these studies do not include the strain-rate dependence of frictional strength on a fault surface that is observed in laboratory studies.

In this study, we incorporate strain-rate softening into the brittle regime of a viscoplastic model of lithospheric stretching. Localized deformation corresponds to zones of high strain rate that develop in response to the rheology and boundary conditions, and are not imposed a priori. Using this formulation, we test the sensitivity of the predicted pattern of deformation to crustal thickness, thermal structure, and the rheologic flow laws describing the crust and mantle. Finally, we compare the results of our numerical simula- 
tions to natural examples of continental and oceanic rifts and discuss the implications for the development and evolution of extensional systems.

\subsection{Model Setup}

At high temperatures geologic materials are observed to flow by dislocation creep, which results in a non-Newtonian power law rheology [Kohlstedt et al., 1995; Kirby, 1983]

$$
\dot{\varepsilon}=A\left(\sigma_{1}-\sigma_{3}\right)^{n} \exp (-Q / R T)
$$

where $\dot{\varepsilon}$ is the uniaxial strain rate, $\sigma_{1}$ and $\sigma_{3}$ are the maximum and minimum principle stresses, $n$ is the power law exponent, $Q$ is the molar activation energy, $A$ is a material strength constant, $T$ is the temperature, and $R$ is the gas constant (see Table 3.1 for list of model parameters). The material parameters $(A, n, Q)$ can be determined from laboratory experiments [e.g., Goetze, 1978] and are approximately independent of stress and temperature. Although the relationship between stress and strain rate is nonlinear, we can define a linearized viscosity law [Chen and Morgan, 1990] by

$$
\tau_{i j}=\sqrt{2} \eta \dot{\varepsilon}_{i j}
$$

where $\tau_{i j}$ is the stress tensor, $\eta$ is the effective viscosity, and $\dot{\varepsilon}_{i j}$ is the strain rate tensor. This linearization leads to an expression for the apparent effective Newtonian viscosity

$$
\eta=B \dot{\varepsilon}_{\mathrm{II}}^{(1-n) / n} \exp (Q / n R T)
$$

where $\dot{\varepsilon}_{\mathrm{II}}$ is the second invariant of the stain rate tensor, and

$$
B=\frac{1}{4}\left(\frac{4}{3 A}\right)^{1 / n}
$$

In the brittle regime, strength is often assumed to be controlled by a frictional resistance law [e.g., Byerlee, 1978; Scholz, 1990] 
Table 3.1: Model Paramters

\begin{tabular}{|c|c|c|c|}
\hline & Definition & Value & Units \\
\hline$g$ & gravitational acceleration & 9.8 & $\mathrm{~m} \mathrm{~s}^{-2}$ \\
\hline$R$ & universal gas constant & 8.3144 & $\mathrm{~J} \mathrm{~mol}^{-1}{ }^{\circ} \mathrm{K}^{-1}$ \\
\hline$T$ & temperature & & ${ }^{\circ} \mathrm{K}$ \\
\hline$n_{c}, n_{m}$ & power law exponent (crust, mantle) & & \\
\hline$A_{c}, A_{m}$ & material strength constant (crust, mantle) & & $\mathrm{MPa}^{-n} \mathrm{~s}^{-2}$ \\
\hline$Q_{c}, Q_{m}$ & molar activation energy (crust, mantle) & & $\mathrm{J} \mathrm{mol}^{-1}$ \\
\hline$\dot{\varepsilon}_{0}$ & reference strain rate & $10^{-14}$ & $s^{-1}$ \\
\hline$\dot{\varepsilon}_{\mathrm{II}}$ & second invariant of the strain rate tensor & & $s^{-1}$ \\
\hline$\eta$ & effective viscosity & & Pa s \\
\hline$\rho_{c}, \rho_{m}$ & density (crust, mantle) & 2700,3300 & $\mathrm{~kg} \mathrm{~m}^{-3}$ \\
\hline$C$ & Coulomb strength coefficient & & \\
\hline$C_{\mathrm{o}}$ & reference Coulomb strength coefficient & & \\
\hline$\theta$ & angle of internal friction & & \\
\hline$\mu$ & reference coefficient of friction & & \\
\hline$\sigma_{\mathrm{o}}$ & cohesive strength & 50 & $\mathrm{MPa}$ \\
\hline$\sigma_{n}$ & lithostatic stress & & $\mathrm{MPa}$ \\
\hline$\gamma$ & strain rate softening coefficient & & \\
\hline$t_{c}$ & crustal thickness & & $\mathrm{km}$ \\
\hline$x, z$ & horizontal and vertical coordinates & & $\mathrm{km}$ \\
\hline$X_{\mathrm{o}}, Z_{\mathrm{o}}$ & horizontal and vertical dimensions of model space & & $\mathrm{km}$ \\
\hline$u_{x}, u_{z}$ & horizontal and vertical velocities & & $\mathrm{m} \mathrm{s}^{-1}$ \\
\hline & depth to $700^{\circ} \mathrm{C}$ isotherm at $x=0$ & & $\mathrm{~km}$ \\
\hline$d \bar{z} / d x$ & slope of the $700^{\circ} \mathrm{C}$ isotherm & & \\
\hline$\frac{\partial I}{\partial z}(x)$ & geothermal gradient & & ${ }^{\circ} \mathrm{K} \mathrm{km}^{-1}$ \\
\hline
\end{tabular}




$$
\tau_{\max }=\sigma_{\mathrm{o}}-C \sigma_{n}
$$

where $\sigma_{\mathrm{o}}$ is the cohesive strength, $C$ is the Coulomb strength coefficient, and $\sigma_{n}$ is approximately equal to the lithostatic stress. $C$ is defined by

$$
C=\frac{\sin (\theta)}{1+\sin (\theta)}
$$

where $\theta$ is the angle of internal friction, which is in turn related to the coefficient of friction, $\mu$, by $\mu=\tan (\theta)$ [Jaeger and Cook, 1979]. Thus for typical values of $\mu$ between 0.6 and $0.85, C=0.34-0.39$. Laboratory experiments have shown that $\mu$ is a function of the sliding velocity, with fault surfaces experiencing lower coefficients of friction at higher strain rates [e.g., Rabinowicz, 1965; Dieterich, 1972]. The degree of strain-rate softening is roughly proportional to the logarithm of the sliding velocity over several orders of magnitude [Scholz, 1990]. To simulate strain-rate softening in the brittle regime, we follow the formulation of Neumann and Zuber [1995] and Montési [2001], expressing the apparent Coulomb strength coefficient, $\mathrm{C}$, as

$$
C=C_{\mathrm{o}}\left(1-\gamma \log _{10}\left(\dot{\varepsilon}_{\mathrm{II}} / \dot{\varepsilon}_{\mathrm{o}}\right)\right)
$$

where $C_{\mathrm{o}}$ is the reference strength coefficient, $\gamma$ is the strain-rate softening coefficient, and $\dot{\varepsilon}_{\mathrm{o}}$ is the reference strain rate.

\subsubsection{Numerical Procedure}

Following the numerical approach of Neumann and Zuber [1995], we use a Lagrangian visco-plastic finite element model to calculate extension in a 2-D vertical cross-section of lithosphere. The model space is discretized into 4-node quadrilateral elements, in which the forces applied to each element, $f^{e}$, are linearly related to the velocities, $u^{e}$, through the element stiffness matrix, $K^{e}$. The penalty method with reduced integration [Reddy, 1984] is used to define the element stiffness matrices, which are then assembled to form the global 
stiffness matrix, $K$. After imposition of the natural (force) and essential (velocity) boundary conditions, the global system of equations, $K u=f$, is solved for the incompressible flow field throughout the model space. The non-linear rheology described above can be solved by iterative linearization of the effective viscosity in each finite element as follows (see also Zuber et al. [1986]; Chen and Morgan [1990]):

1) Calculate the initial effective viscosity for each element from Eq. 3.3 based on the prescribed temperature field, $T(x, z)$, and a uniform reference strain rate, $\dot{\varepsilon}_{0}$.

2) For elements in which the resulting maximum principle shear stress, $\tau_{i j}=\sqrt{2} \eta \dot{\varepsilon}_{\mathrm{II}}$, is greater than the frictional failure criterion, $\tau_{\max }$, reset the effective viscosity of the element to $\eta=\tau_{\max } / \sqrt{2} \dot{\varepsilon}_{\mathrm{II}}$.

3) Form global stiffness matrix, $K$, prescribe velocity boundary conditions and gravitational body forces, and solve the finite element equation, $K u=f$, for the velocities $u$.

4) Based on the velocities calculated in Step 3, recalculate the strain rate in each element and use Eq. 3.3 to update the element effective viscosities.

5) Repeat Steps 2-4 until the desired level of convergence is reached.

6) Deform grid by applying nodal velocities, $u$, over a reference time step $\Delta t$.

7) Repeat Steps $2-6$ until $1 \%$ total strain is achieved.

The results of the numerical experiments presented here should be treated as a proxy for the initial pattern of deformation that will develop for a given set of thermal and rheologic parameters, rather than as a method to study the evolution of individual faults over geologic time. The rotation of fault blocks in highly extended terraines can generate large flexural stresses [e.g., Forsyth, 1992; Buck, 1993] that are not accounted for in the visco-plastic formulation described above. By limiting our numerical experiments to a maximum of $1 \%$ 
total extension, we can safely ignore these elastic stresses and also eliminate numerical inaccuracies associated with the distortion of model elements.

The numerical model setup is illustrated in Fig. 3-1. A uniform horizontal velocity, $u_{x}=U$, is imposed on the right hand side of the model space $\left(x=X_{\mathrm{o}}\right)$. For numerical efficiency, a symmetry condition is placed on the left-hand side of the model $(x=0)$ such that $u_{x}=0$ and $\tau_{x z}=0$. The bottom boundary $\left(z=Z_{\mathrm{o}}\right)$ is assumed to be free-slip with no vertical velocity, while the top boundary $(z=0)$ is stress free. The model dimensions, $X_{\mathrm{o}}=55 \mathrm{~km}$ and $Z_{\mathrm{o}}=60 \mathrm{~km}$, are specified to insure that the boundaries do no influence the final solution, and for each numerical experiment the finite element grid is adjusted to give maximum resolution where strain is concentrated. The crustal thickness, $t_{c}$, is defined to be constant across the model space, with the crust and mantle material parameters given by $A_{c}, n_{c}, Q_{c}$ and $A_{m}, n_{m}, Q_{m}$, respectively. The reference strain rate, $\dot{\varepsilon}_{o}$, is defined to be $10^{-14} \mathrm{~s}^{-1}$, and the strain rates reported here are referenced to this value.

The temperature field is defined by two parameters, the depth to the $700^{\circ} \mathrm{C}$ isotherm at $x=0, \bar{z}_{\mathrm{o}}$, and the slope of the $700^{\circ} \mathrm{C}$ isotherm, $d \bar{z} / d x$ (Fig. 3-1). Above the $700^{\circ} \mathrm{C}$ isotherm, temperature is assumed to be controlled by conductive cooling and is linear with depth,

$$
T(x, z \leq \bar{z})=z \frac{\partial T}{\partial z}(x)
$$

For depths greater than $\bar{z}$, temperature follows a steady-state half-space cooling model,

$$
T(x, z>\bar{z})=700+\left(T_{m}-700\right) \operatorname{erf}\left(\frac{\sqrt{\pi} z}{2\left(T_{m}-700\right)} \frac{\partial T}{\partial z}(x)\right)
$$

where $T_{m}$ is the mantle temperature. The geothermal gradient, $\frac{\partial T}{\partial z}(x)$, is a function of $x$ and is given by

$$
\frac{\partial T}{\partial z}(x)=\frac{700}{\bar{z}_{\mathrm{o}}+\frac{d \bar{z}}{d x} x}
$$




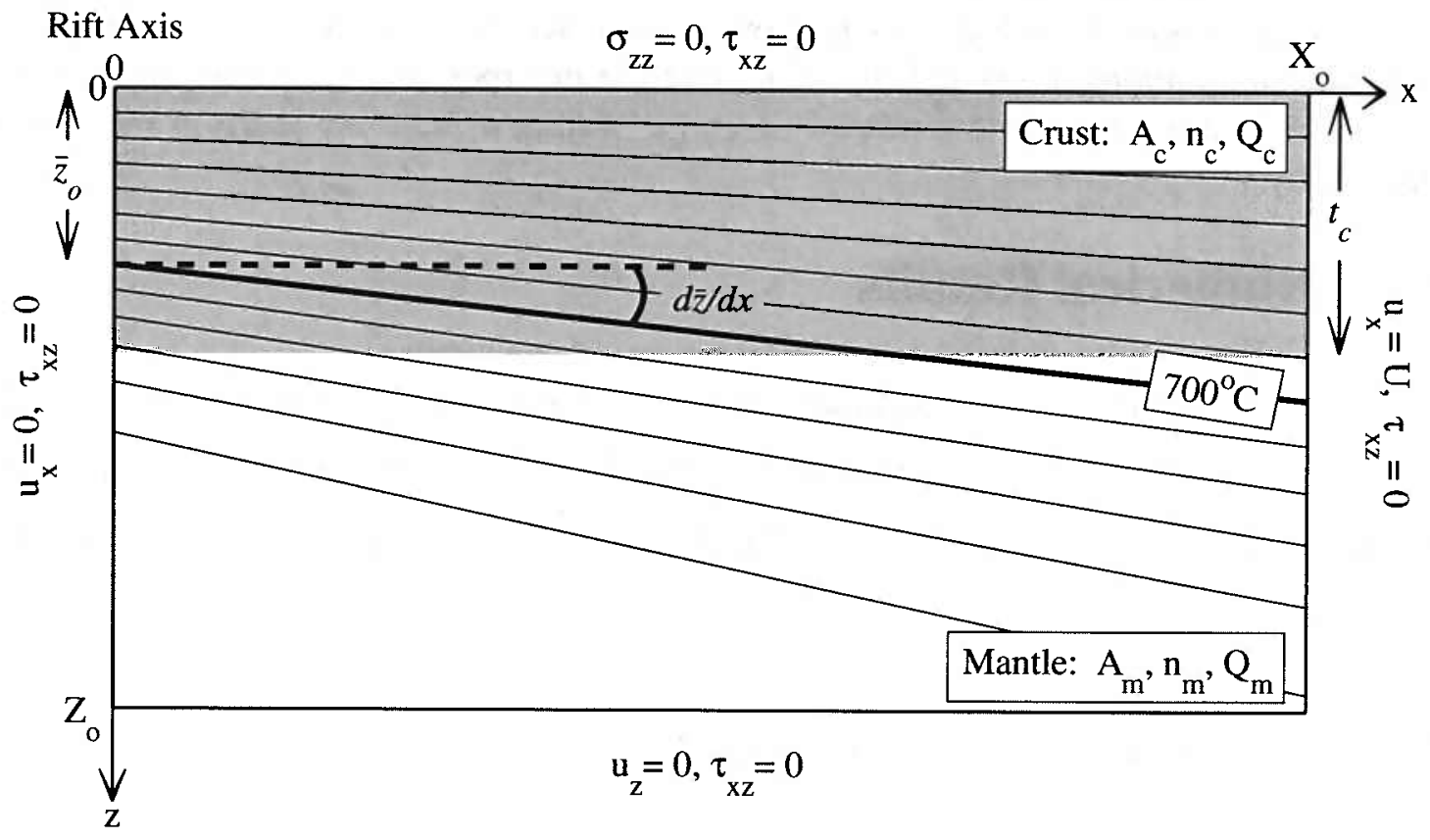

Figure 3-1: Model setup for numerical simulations of lithospheric stretching. The model space is symmetric about the rifting axis $(x=0)$, with dimensions $X_{\mathrm{o}}$ and $Z_{0}$. A uniform horizontal velocity, $u_{x}=U$ is applied to the right hand side of the model space, and extension is continued until $1 \%$ total strain is achieved. Crustal thickness, $t_{c}$, is constant across the model space, with the crust and mantle material parameters given by $A_{c}, n_{c}, Q_{c}$ and $A_{m}, n_{m}, Q_{m}$, respectively. The temperature field is described by the depth to the $700^{\circ} \mathrm{C}$ isotherm at the rift axis, $\bar{z}_{\mathrm{o}}$, and the slope of the $700^{\circ} \mathrm{C}$ isotherm, $d \bar{z} / d x$. 
Figure 3-2: (facing page) Numerical simulations illustrating the importance of the strainrate softening coefficient, $\gamma$, on localizing deformation into narrow fault-like shear zones. (a) $\gamma=0$. (b) $\gamma=0.15$. The deformed finite element grid (right) is shown after $1 \%$ total strain, with colors representing the second invarient of the strain-rate tensor normalized to $\dot{\varepsilon}_{\mathrm{o}}$ in each element. Only upper left portion of model is shown; overall model dimensions are $X_{\mathrm{o}}=55 \mathrm{~km}$ and $Z_{\mathrm{o}}=60 \mathrm{~km}$. The initial temperature field $\left(\bar{z}_{\mathrm{o}}=15 \mathrm{~km}, d \bar{z} / d x\right.$ $=0.15$ ) is shown on the left, with temperature contour interval of $100^{\circ} \mathrm{C}$. A $20-\mathrm{km}$ thick crust is assumed (gray box on left; bold lines on right). The experimental flow laws 3 [Caristan, 1982] and 7 [Goetze, 1978] from Table 2 are used for the crust and mantle, respectively. Location of Fig. 3-4 is shown by dashed box. (c) Surface topography of numerical simulations in (a) and (b). Note that the incorporation of strain-rate softening focuses high strain rates into a narrow zone, generating a fault-like scarp in the surface topography.

\subsection{Numerical Results}

In this section we present a series of numerical experiments to evaluate the sensitivity of the predicted style of lithospheric deformation to the degree of strain-rate softening, regional thermal structure, crustal thickness, and the rheologic flow laws used to describe the crust and mantle.

\subsubsection{Strain-Rate Softening Coefficient}

In order to examine the effect of strain-rate softening on the pattern of deformation during extension, we tested a range of values for the strain-rate softening coefficient, $\gamma$, between 0 and 0.2 . Figs. $3-2 a$ and $3-2 b$ show the deformed finite element grid for $\gamma$ values of 0 and 0.15 , respectively. When strain-rate softening is ignored $(\gamma=0)$, a wide diffuse region of moderately high strain-rates is calculated to develop near the rift axis. In contrast, when $\gamma$ $=0.15$, high strain rates are focused into a narrow zone of localized deformation, resulting in a fault-like scarp in the surface topography (Fig. 3-2c).

Fig. 3-3 illustrates the degree to which the strain-rate softening coefficient controls both the calculated width of the deformation zone and the maximum value of strain rate at $z=0$ $\mathrm{km}$. Based on this analysis, it appears that values of $\gamma \geq 0.10$ are sufficient to localize deformation in a narrow shear zone analogous to a fault (refered to hereafter as a "fault zone"), and we choose $\gamma=0.15$ for the remainder of the numerical experiments presented 

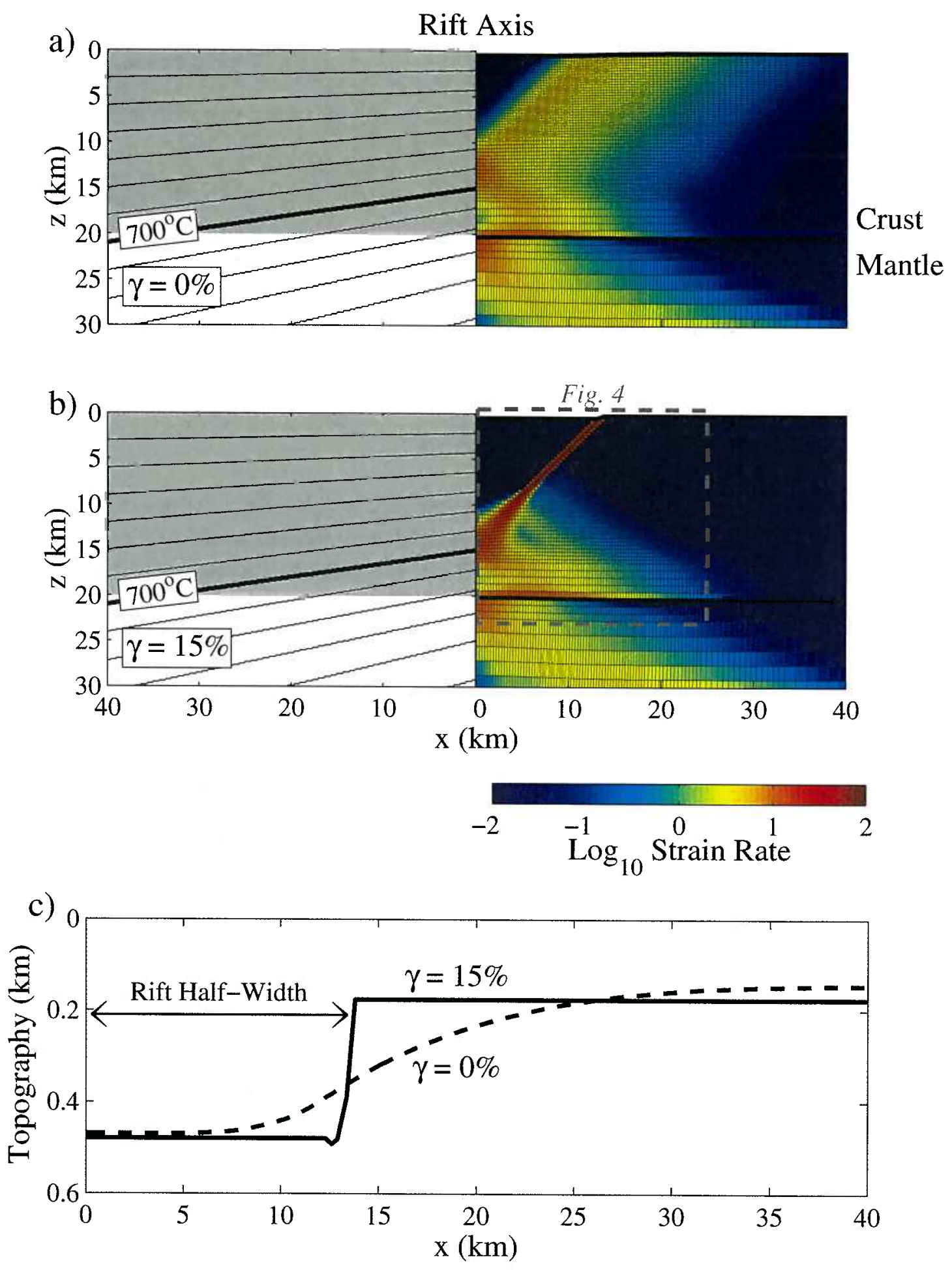


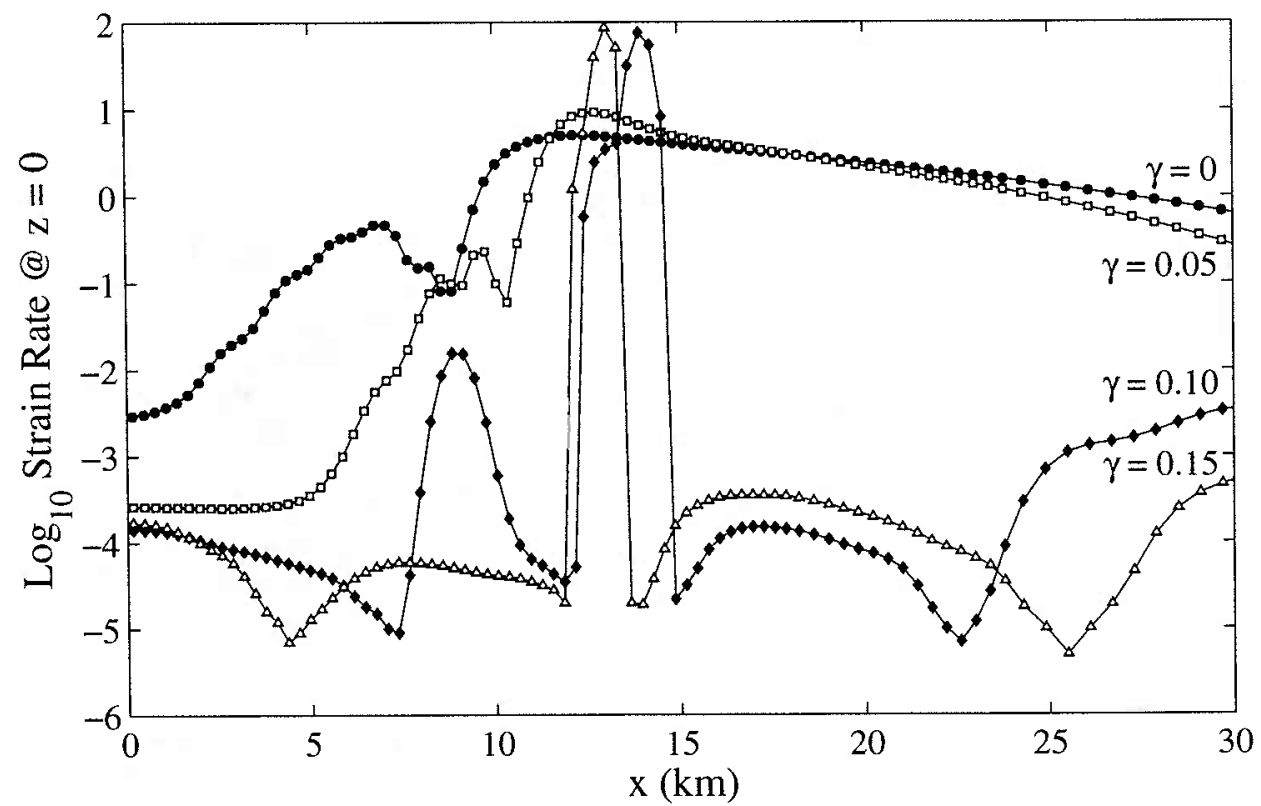

Figure 3-3: Comparison of the calculated strain-rate at $z=0 \mathrm{~km}$ for $\gamma$ values ranging from 0 to 0.15 . Larger values of $\gamma$ result in a narrower deformation zone and a higher maximum strain-rate within the zone. Values of $\gamma \geq 0.10$ are sufficient to localize deformation into a narrow region analogous to a fault zone. Note that the variations in strain-rate with magnitudes $<10^{-3} \mathrm{~s}^{-1}$ are numerical effects and do not impact the final model solutions.

in this study. For the $\sim 2$ order of magnitude increase in $\dot{\varepsilon}_{\text {II }}$ relative to $\dot{\varepsilon}_{\mathrm{o}}$ within the fault zone shown in Fig. $3-2 b, \gamma=0.15$ would result in a $\sim 30 \%$ reduction of the apparent strength coefficient, $C$, and a $\sim 50 \%$ reduction in the coefficient of friction, $\mu$.

It is important to note that the predicted pattern of deformation develops in response to the initial temperature field, crustal thickness, and rheologic flow laws, and is not imposed a priori. Thus, the results of our numerical simulations can be used to predict the preferred location of fault formation for an initial set of thermal and rheologic conditions. Neumann and Zuber [1995] also used a strain-rate softening rheology in the brittle regime to model the development of fault-like shear zones in an extending lithosphere. However, they considered only a horizontally homogeneous plate exposed to a $0.5 \mathrm{~km}$ perturbation in surface topography at the rift-axis. In the models presented here, the initial topography of the model space is uniform, and the deformation pattern develops from the prescribed horizontal variations in the temperature field. 


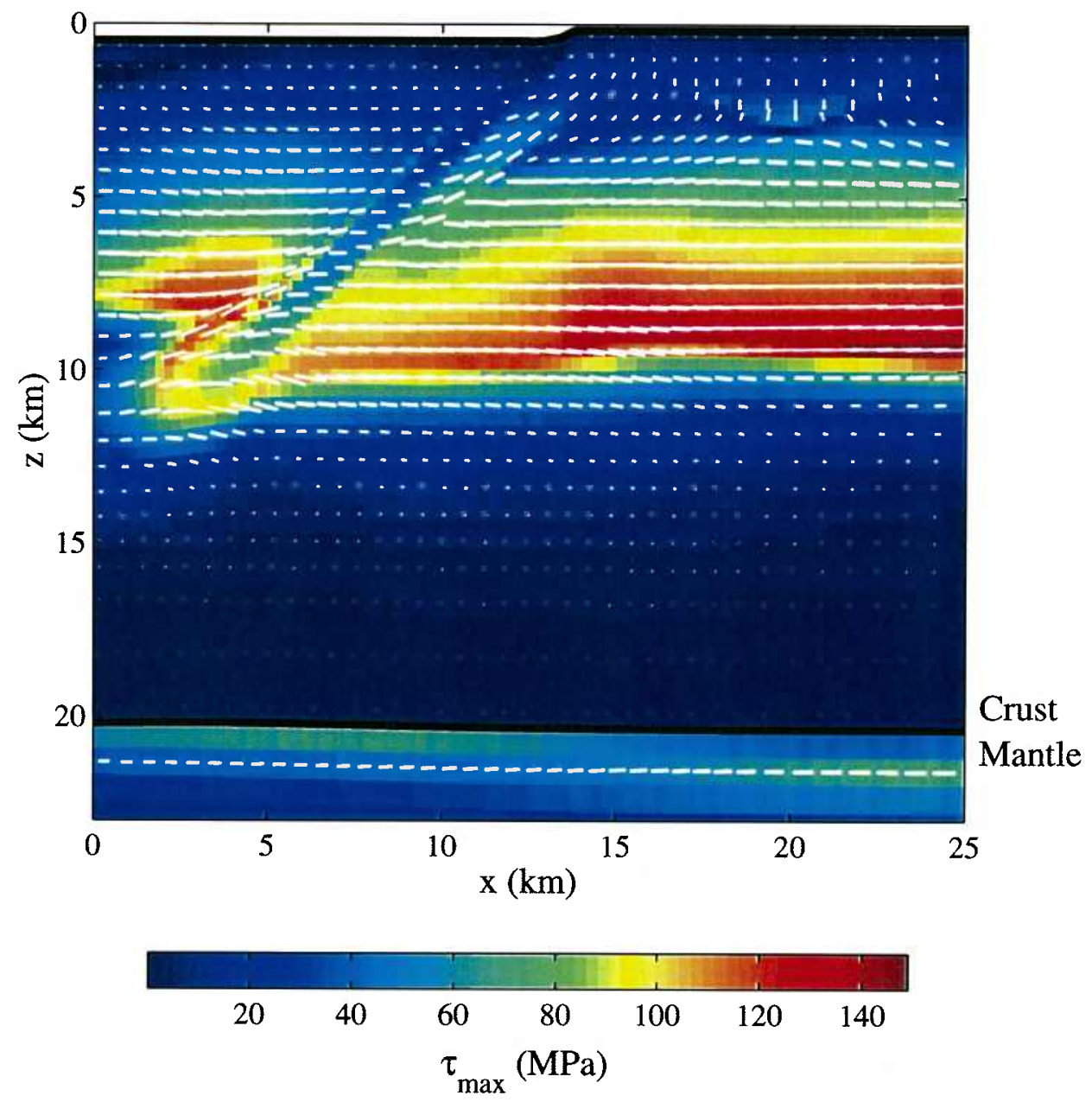

Figure 3-4: Stress field corresponding to the numerical simulation shown in Fig. 3-2b. Colors indicate the magnitude of $\tau_{\max }$ in each element and white lines show the direction of maximum tensile stress with length scaled to the magnitude of $\tau_{\max }$. Thick black line represents crust-mantle boundary. Note that $\tau_{\max }$ reaches a maximum value near the base of the brittle upper crust and increases again in the upper mantle. Note also that the region of high strain rate in the inclined "fault like" shear band corresponds to an area of rotated maximum tensile stress direction and a decrease in the magnitude of $\tau_{\max }$. 
Figure 3-5: (facing page) Numerical simulations illustrating the importance of the horizontal temperature gradient in localizing deformation on a regional scale. (a), (b), (c), and (d) show deformed finite element grids after $1 \%$ total strain for $\bar{z}_{0}=5 \mathrm{~km}$ and $d \bar{z} / d x$ $=0,0.01,0.05,0.15$, respectively. A 6-km thick crust is assumed (bold lines), and the flow laws 3 [Caristan, 1982] and 7 [Goetze, 1978] from Table 2 are used for the crust and mantle, respectively. When no lateral temperature gradient is present (a), deformation is distributed between several sets of conjugate normal faults. However, when $d \bar{z} / d x>0.10$ (d), deformation is localized on one major fault near the rift axis.

Fig. 3-4 shows the stress field around the fault zone in Fig. 3-2b. The minimum width of the fault zone is controlled by the spacing of the finite element grid, and is $\sim 4$ elements wide. The effective viscosity of the elements within the fault zone is typically 4-6 orders of magnitude lower than in the adjacent regions. High stresses ( $\left.\tau_{\max }>100 \mathrm{MPa}\right)$ are predicted near the base of the brittle upper crust and in the upper mantle, while the ductile lower crust is characterized by values of $\tau_{\max }<20 \mathrm{MPa}$. Within the fault zone, stresses are also low due to the low effective viscosity of these elements. This is significant because it implies that our modeling technique is only capable of generating "weak" faults. The zones adjacent to the fault, however, are characterized by elevated stresses. In the footwall the direction of maximum tensile stress is rotated from horizontal into the direction of deformation. This prediction is similar to modeling by Chéry [2001] of the stress field around a weak fault zone in a visco-elastic space.

\subsubsection{Horizontal Temperature Gradient}

Extension in continental lithosphere typically results in the formation of either wide (e.g., Basin and Range) or narrow (e.g., Rhinegraben, Baikal) rift zones. Models to distinguish between these two styles of rifting have focused on the thermal evolution of the lithosphere through time. In these models thermal state is the result of equilibrium between the relative rates of heat advection due to athenospheric upwelling and thermal diffusion through increased surface heat flow [e.g., England, 1983; Kusznir and Park, 1987]. Narrow rift zones are thought to result from runaway lithospheric thinning caused by rapid extension, while wide rifts develop when strain rates are low and thermal diffusion dominates [Sonder and 

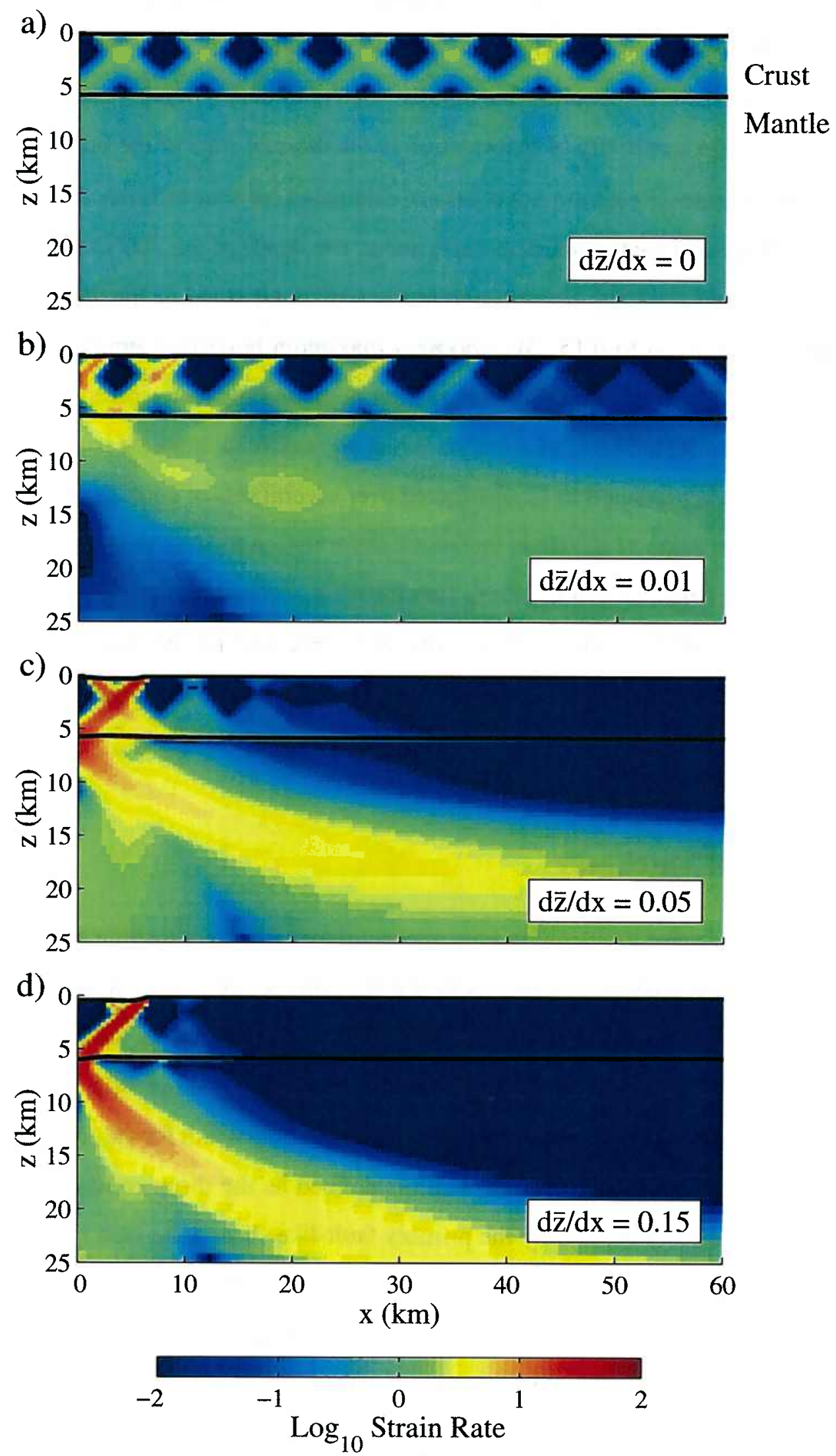
England, 1989]. Because our numerical experiments are limited to small amounts of total strain, we cannot examine the long-term evolution of rift zones. However, assuming the pattern of deformation will equilibriate rapidly relative to the evolving thermal conditions, we can examine the sensitivity of deformation to the thermal state of the lithosphere.

Fig. 3-5 illustrates the deformation pattern calculated for four different horizontal temperature gradients. In each numerical experiment, the depth of the $700^{\circ} \mathrm{C}$ isotherm at the rift axis, $\bar{z}_{0}$, is held constant at $5 \mathrm{~km}$, while the horizontal slope of the $700^{\circ} \mathrm{C}$ isotherm, $d \bar{z} / d x$, is varied from 0 to 0.15 . We choose a maximum horizontal gradient of 0.15 because it corresponds to the approximate gradient predicted for slow-spreading mid-ocean ridge with a spreading half-rate of $1 \mathrm{~cm} / \mathrm{yr}$. When no horizontal gradient is present $(d \bar{z} / d x$ $=0$ ), deformation is predicted to be distributed over several sets of conjugate normal faults (Fig 3-5a). As the value of $d \bar{z} / d x$ is increased, deformation focuses near the thinnest part of the lithosphere (Figs. 3-5b and 3-5c). This focusing is characterized by fewer predicted faults, higher strain rates on individual faults, and a preferential dip direction toward the rift axis. Values of $d \bar{z} / d x>0.10$ result in focusing onto a single fault centered near the rift axis (Fig. 3-5d).

\subsubsection{Crustal Thickness}

Crustal thickness is also predicted to play an important role in controlling the style of deformation during extension. Crustal rocks are typically weaker than mantle rocks [e.g., Kirby, 1983]. Therefore, under a constant set of thermal conditions the brittle-ductile transition will tend to be shallower in rifts with thick crust than in those with thin crust. We investigated the influence of crustal thickness, $t_{c}$, on rift half-width for values of $t_{c}$ ranging from 0 to $40 \mathrm{~km}$ (Fig. 3-6). Rift half-width is defined as the distance from the rift axis to the topographic scarp formed by the primary fault-like shear zone (see Fig. 3-2c). For small values of $t_{c}$, the entire crustal layer is predicted to behave brittlely and the resultant rift half-width is independent of crustal thickness. However, once $t_{c}$ is large enough for a ductile region to form in the lower crust, rift half-width is calculated to decrease with 


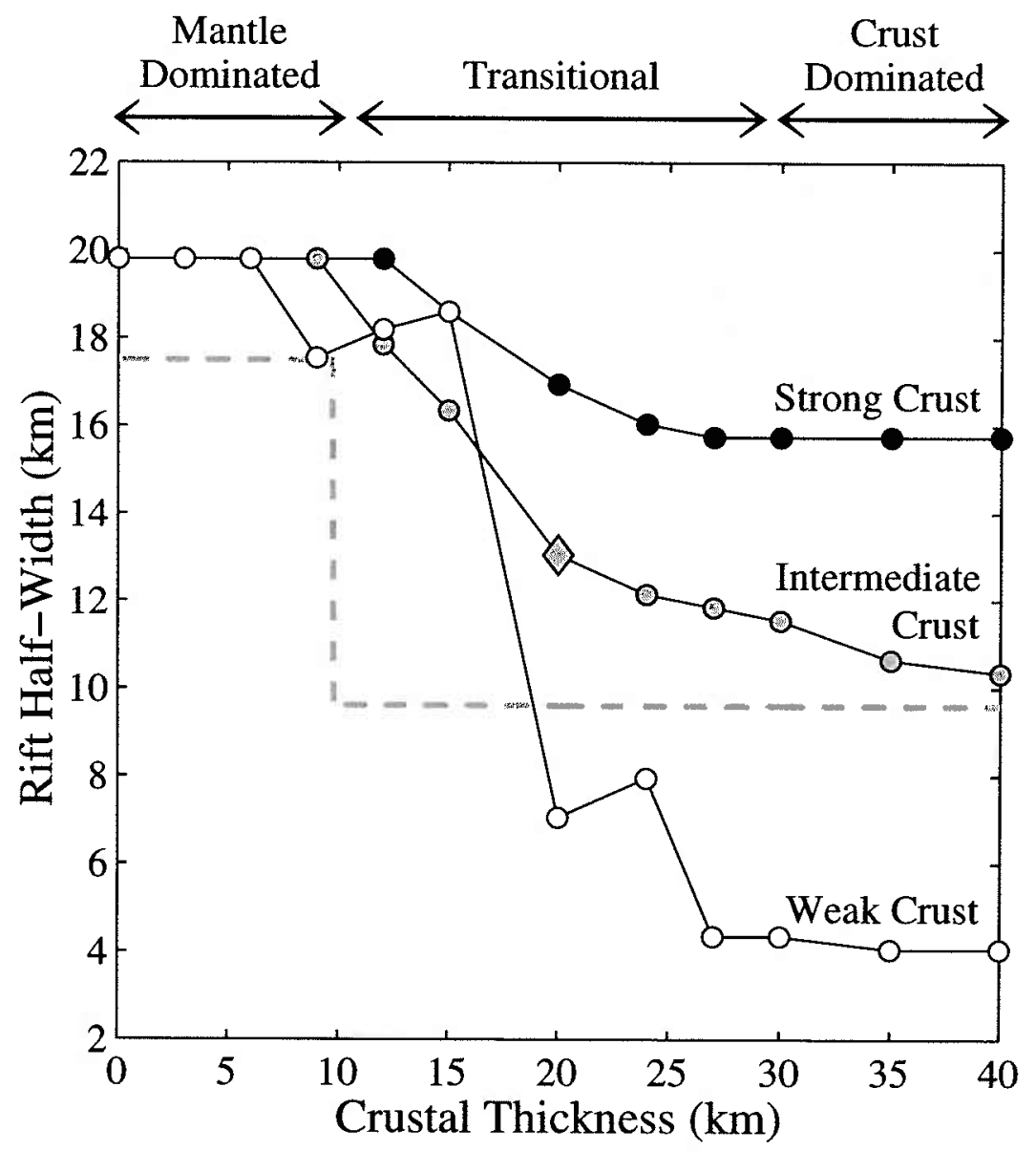

Figure 3-6: Effect of crustal thickness on predicted rift half-width. Each dot represents the results of one numerical experiment. The initial temperature field is defined by $\bar{z}_{0}=15 \mathrm{~km}$, $d \bar{z} / d x=0.15$, resulting in a geothermal gradient of $46^{\circ} \mathrm{K} / \mathrm{km}$ at $x=0 \mathrm{~km}$. Calculations were performed using the crustal flow laws for a weak (flow law 2 [Hansen and Carter, 1983] in Table 3.2, white circles), intermediate (flow law 3 [Caristan, 1982], grey circles), and strong (flow law 6 [Mackwell et al., 1998], black circles) rheology. Grey diamond represents model run shown in Fig. 3-2b. Rift half-width is defined as the distance from the rift axis to the calculated topographic scarp formed by the primary fault zone. All calculations were performed with $d \bar{z} / d x=0.15$ (see Fig. 3-2c). The dashed line illustrates the prediction of the analytical solution of Shaw and Lin [1996] for flow law 3. Note that the change from mantle- to crust-dominated deformation does not occur at a critical crustal thickness as predicted by Shaw and Lin [1996], but rather over a transitional range of crustal thicknesses. The strong rheology results in a rift half-width in the crust-dominated regime that is $\sim 1.5$ and $\sim 4$ times that predicted by the intermediate and weak rheologies, respectively. 
Figure 3-7: (facing page) Numerical simulations illustrating the tranistion from mantle- to crust-dominated deformation. (a), (b), and (c) show the deformed finite element grid after $1 \%$ total strain for $t_{c}=6,12$, and $27 \mathrm{~km}$, respectively. Colors indicate the magnitude of $\tau_{\text {max }}$ on left and normalized strain-rate on right. $\mathrm{C}$ and $\mathrm{M}$ mark the base of the crust and top of the mantle, respectively. The initial temperature field is defined by $\bar{z}_{\mathrm{o}}=15 \mathrm{~km}, d \bar{z} / d x=$ 0.15 , resulting in a geothermal gradient of $46^{\circ} \mathrm{K} / \mathrm{km}$ at $x=0 \mathrm{~km}$. The experimental flow laws 2 [Hansen and Carter, 1983] and 7 [Goetze, 1978] from Table 2 are used for the crust and mantle, respectively. Note in (b) that deformation in the upper crust is predicted to be coupled to the mantle even when a ductile layer is present in the lower crust.

increasing crustal thickness. Eventually, $t_{c}$ becomes sufficiently large that rift half-width no longer decreases and remains constant for further increases in $t_{c}$.

We term the two regimes in which rift half-width is independent of crustal thickness mantle-dominated $\left(t_{c}\right.$ small, rift half-width large) and crust-dominated ( $t_{c}$ large, rift halfwidth small) deformation, respectively. The range of crustal thicknesses over which deformation changes from mantle-dominated to crust-dominated is refered to as the transition zone (see Fig. 3-6). Gerbault et al. [1999] described a similar transition in compressional systems from faulting in a coupled crust-mantle layer to faulting in only the crustal layer. Fig. 3-7 shows three numerical experiments illustrating the transition from mantleto crust-dominated deformation. Note that the transition to crust-dominated deformation is complete only when stress accumulation in the upper crust is much greater than stress accumulation in the upper mantle. The numerical experiments presented in Sec. 3.4 and 3.5 allow us to constrain how the transition from mantle- to crust-dominated deformation varies as a function of both crustal rheology and thermal state.

\subsubsection{Crustal Rheology}

Within the ductile deformation regime, the strength of the crust at a given temperature and strain rate is defined by the material parameters $A_{c}, n_{c}$, and $Q_{c}$ in Eq. 3.1. The flow laws for a wide range of crustal and mantle rocks have been determined in experimental studies. In addition to mineralogy (e.g., quartz vs. feldspar aggregates), water content plays an important role in controlling the strength of a material [Tullis and Yund, 1980]. 

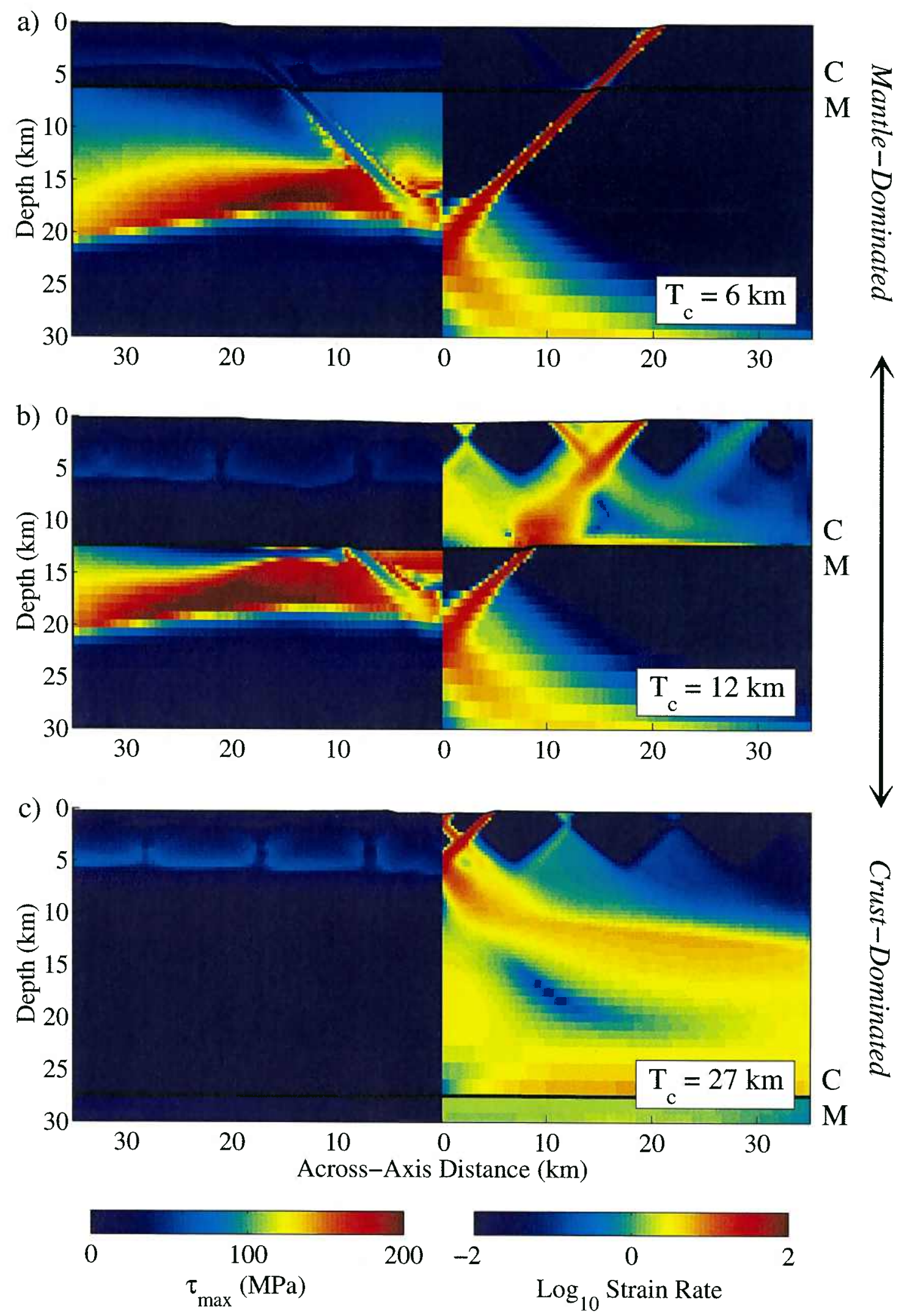
Table 3.2: Experimental Flow Laws for Crustal and Mantle Rocks

\begin{tabular}{|c|c|c|c|c|c|}
\hline Curve & Material & $\mathrm{A}, \mathrm{MPa}^{-n} \mathrm{~s}^{-1}$ & $\mathrm{n}$ & $\mathrm{Q}, \mathrm{kJ} \mathrm{mol}^{-1}$ & Reference* \\
\hline \multicolumn{6}{|c|}{ Weak Crust } \\
\hline 1 & Heavitree quartzite (wet) & $2.91 \times 10^{-3}$ & 1.8 & 151 & J84 \\
\hline 2 & Westerly granite (wet) & $2.00 \times 10^{-4}$ & 1.9 & 140 & $\mathrm{HC} 83$ \\
\hline \multicolumn{6}{|c|}{ Intermediate Crust } \\
\hline 3 & Maryland diabase (wet) & $6.12 \times 10^{-2}$ & 3.05 & 276 & C82 \\
\hline 4 & Westerly granite (dry) & $2.00 \times 10^{-6}$ & 3.3 & 186 & $\mathrm{HC} 83$ \\
\hline 5 & Heavitree quartzite (dry) & $5.00 \times 10^{-6}$ & 3.2 & 220 & KT84 \\
\hline \multicolumn{6}{|c|}{ Strong Crust } \\
\hline 6 & Maryland diabase (dry) & $1.90 \times 10^{2}$ & 4.7 & 485 & M98 \\
\hline \multicolumn{6}{|c|}{ Mantle } \\
\hline 7 & Dunite (dry) & $1.00 \times 10^{3}$ & 3 & 520 & G78 \\
\hline
\end{tabular}

For example, in samples of diabase the presence of water can result in a 2-3 order of magnitude decrease in effective viscosity [Hirth et al., 1998]. The use of a dry rheology may be particularly relevant in environments that experience volcanism during rifting, as water is an incompatible element and likely to be preferentially extracted during the initial stages of melting. Table 3.2 lists experimental flow laws for several different wet and dry crustal rheologies, which we classify as weak, intermediate, and strong. Fig. 3-8 shows the effective viscosity versus depth for these rheologies assuming $\bar{z}_{\mathrm{o}}=15 \mathrm{~km}$ (or a geothermal gradient of $46^{\circ} \mathrm{K} / \mathrm{km}$ at $x=0 \mathrm{~km}$ ).

In Fig. 3-6 we illustrate the effect of crustal rheology on rift half-width for a weak (flow law 2 [Hansen and Carter, 1983] in Table 3.2), intermediate (flow law 3 [Caristan, 1982]), and strong (flow law 6 [Mackwell et al., 1998]) rheology. All three rheologies predict the same rift half-width in the mantle-dominated deformation regime. However, the transition between mantle- and crust-dominated deformation is predicted to begin at a 


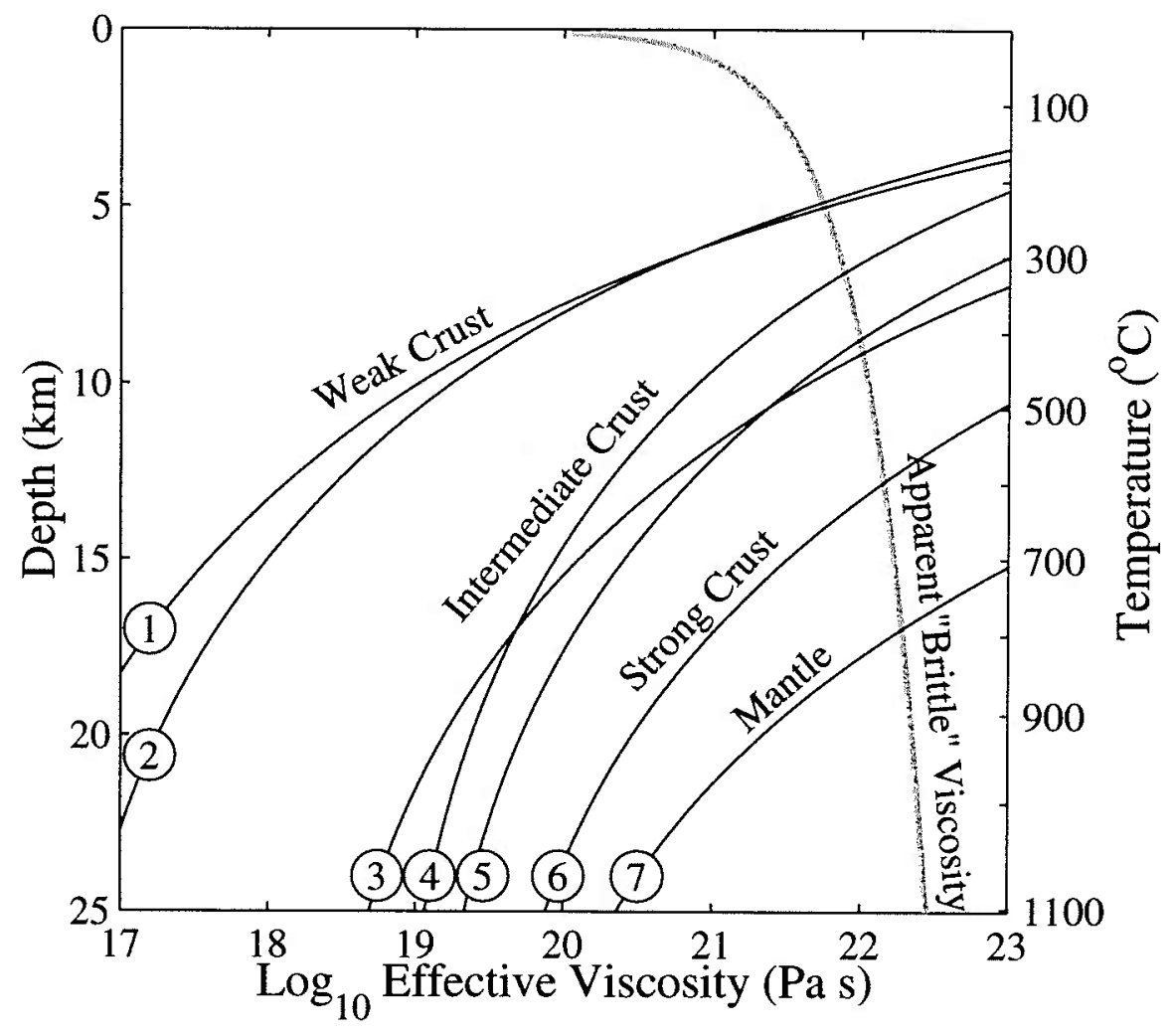

Figure 3-8: Effective viscosity as a function of depth for the experimentally determined flow laws given in Table 2 . Solid grey line illustrates apparent "brittle" viscosity calculated from the frictional resistance law in Eq. 3.5. Temperatures are calculated for $\bar{z}_{0 .}=15 \mathrm{~km}$, corresponding to a geothermal gradient of $46^{\circ} \mathrm{K} / \mathrm{km}$. Reference strain-rate of $10^{-14} \mathrm{~s}^{-1}$ was used for all calculations.

slightly larger crustal thickness for the strong rheology than for the intermediate or weak rheologies. Further, the strong rheology results a rift half-width for the crust-dominated regime that is $\sim 1.5$ times greater than the value predicted by the intermediate rheology and $\sim 4$ times greater than that predicted by the weak rheology. In reality, the lower continental crust is most likely composed of a feldspar dominated mineralogy [Rutter and Brodie, 1992]. Thus, model results corresponding to either the strong or intermediate rheologies are most applicable to continental extensional environments. 


\subsubsection{Geothermal Gradient}

In Sec. 3.2, horizontal variations in temperature were shown to play an important role in localizing deformation on a regional scale. Here we illustrate how the vertical geother-

mal gradient, $\frac{\partial T}{\partial z}(x)$, affects rift half-width for values of $\frac{\partial T}{\partial z}(x=0)$ ranging from 25 to $140^{\circ} \mathrm{K} / \mathrm{km}$ (Fig. 3-9). Our numerical results predict that as the geothermal gradient decreases, rift half-width increases. Moreover, smaller geothermal gradients also result in larger changes in the predicted rift half-width between the mantle- and crust-dominated deformation regimes.

Fig. 3-9 provides a summary of the combined effects of crustal thickness, crustal rheology, and geothermal gradient on rift half-width. In general, when crustal thickness is small and deformation is mantle-dominated, rift half-width is predicted to be controlled primarily by the geothermal gradient. In contrast, when crustal thickness is large and deformation is crust-dominated, the calculated rift half-width is a function of both crustal rheology and geothermal gradient.

\subsection{Discussion}

\subsubsection{Strain-rate vs. Strain Softening}

While previous modeling studies have examined the effect of strain softening on strain localization in the lithosphere [Lavier et al., 2000; Poliakov and Buck, 1998; Frederiksen and Braun, 2001], the goal of this study is to investigate strain-rate softening as a mechanism for fault generation in a continuum model. Our choice of weakening mechanisms was motivated by several considerations. First, laboratory studies have shown that frictional strength on a fault surface is a strong function of sliding velocity [Tullis and Weeks, 1986], as well as the strain and loading history [Dieterich, 1979; Ruina, 1983]. Furthermore, a recent instability analysis by Montési and Zuber [2002] showed that velocity weakening on faults can result in a highly negative inverse effective stress exponent, indicative of efficient strain localization. We note that velocity weakening is not the only mechanism characterized by 


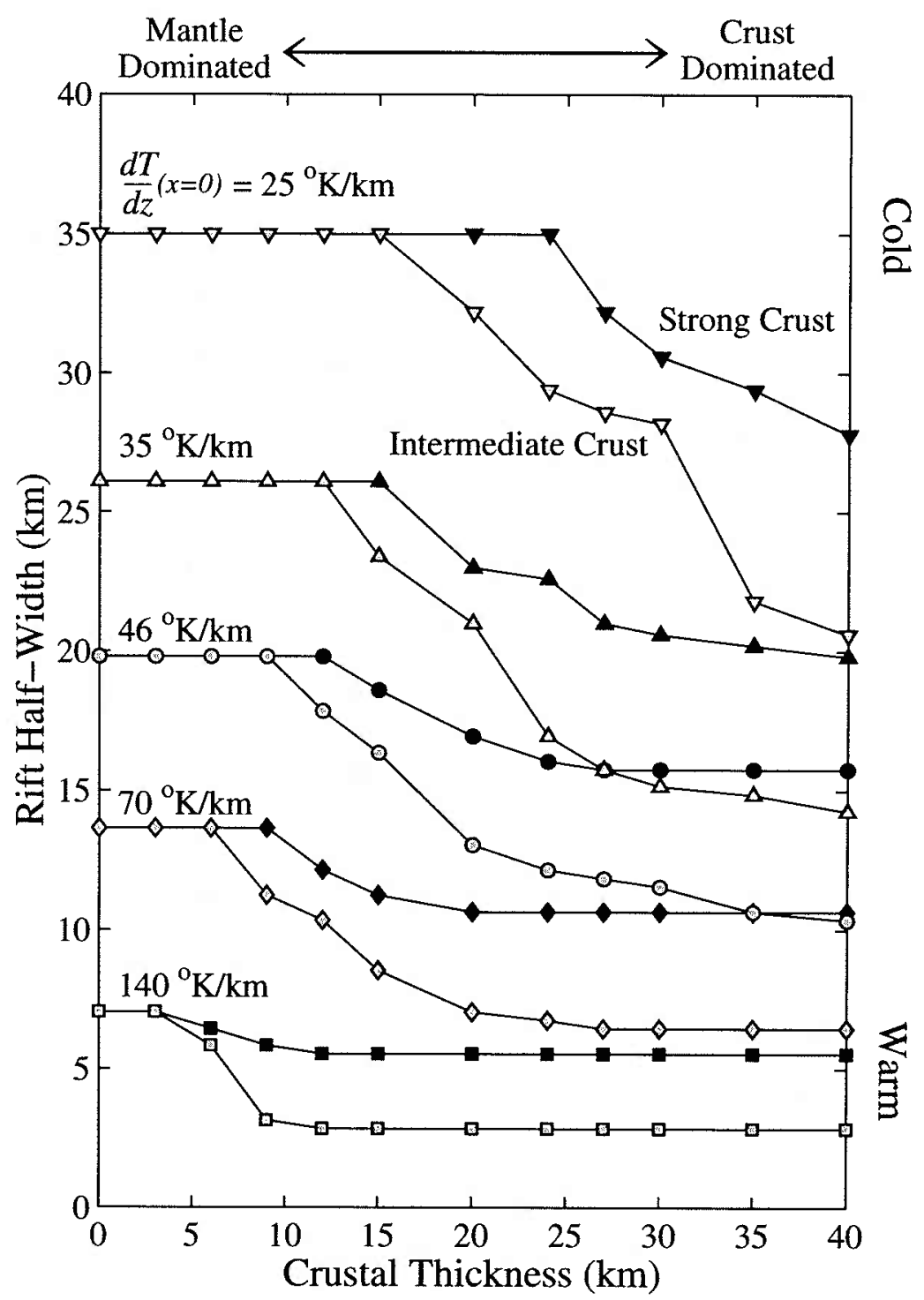

Figure 3-9: Effect of the geothermal gradient on the predicted rift half-width. Geothermal gradients of 140 (squares), 70 (diamonds), 46 (circles), 35 (triangles), and $25^{\circ} \mathrm{K} / \mathrm{km}$ (inverted triangles) corresponding to $\bar{z}_{0}=5,10,15,20,28 \mathrm{~km}$, respectively. Each symbol represents the results of one numerical experiment. Black and grey symbols represent results for strong (flow law 6 [Mackwell et al., 1998] in Table 3.2) and intermediate (flow law 3 [Caristan, 1982]) rheologies, respectively. Rift half-width is predicted to increase with decreasing geothermal gradient. Note that smaller geothermal gradients also result in larger changes in rift half-width with increasing crustal thickness. 
a negative inverse stress exponent. Cohesion loss, shear heating, and grain-size feedbacks are also predicted to produce localization [Montési and Zuber, 2002]. Future models can incorporate these additional mechanisms in order to test the relative importance of each on the pattern of lithospheric deformation.

A strain-rate softening rheology can lead to more rapid strain localization than strain softening, resulting in more "fault-like" features. Rheologies in which softening scales with the total accumulated strain [e.g., Frederiksen and Braun, 2001] typically result in wide $(10 \mathrm{~s} \mathrm{~km})$ shear zones because regions that have undergone a finite amount of strain will remain weak. In contrast, strain-rate softening allows areas that are no longer deforming to heal and strengthen, enhancing strain localization.

Poliakov and Buck [1998] and Lavier et al. [2000] were able to produce fault-like behavior assuming strain softening due to cohesion loss on a fault surface. However, this formulation results in such rapid strain localization that a relatively narrow rift zone is generated even for a plate with no horizontal strength variations [Lavier et al., 2000]. In contrast, the strain-rate softening rheology presented here is capable of predicting either distributed or localized faulting depending on the regional temperature field (Fig. 3-5). In reality, both strain and strain-rate softening are likely to be important mechanisms for strain localization in the lithosphere [Montési and Zuber, 2002], and further modeling efforts are necessary to examine the interplay between these two processes.

\subsubsection{Crust- vs. Mantle-Dominated Deformation}

Most previous studies of normal fault initiation have treated the upper crust as an elasticplastic layer floating on an inviscid substrate and ignored the effects of a strong upper mantle at depth [e.g., Vening Meinesz, 1950; Bott, 1981; Forsyth, 1992; Buck, 1993; Shaw and Lin, 1996; Lavier et al., 2000]. Results of instability analyses [e.g., Zuber et al., 1986] and the numerical calculations presented here clearly suggest that the mantle may play a significant role in controlling deformation in the upper crust even in the presence of a ductile lower crustal layer. Of particular interest is the range of crustal thicknesses, over 

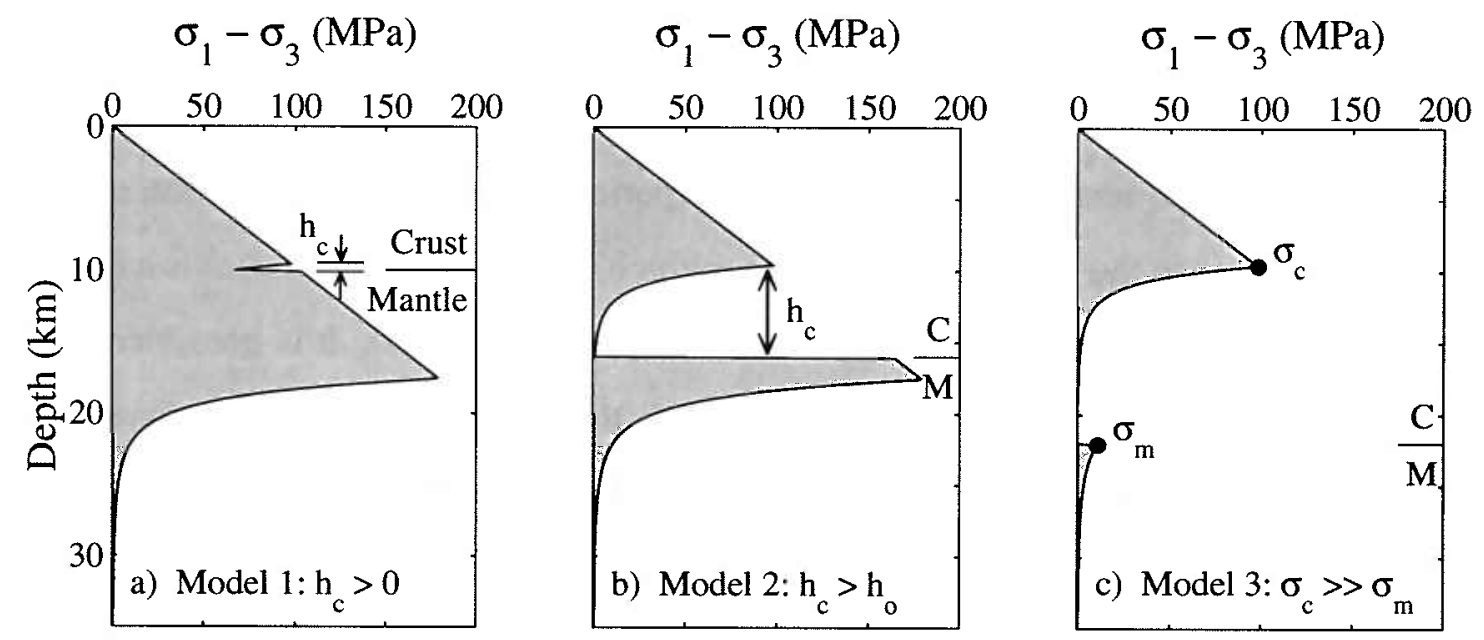

Figure 3-10: Three potential models to explain the transition from mantle-dominated to crust-dominated deformation in Figs. 3-6 and 3-9. (a) Model 1: Requires only the presence of a ductile lower crustal channel, with thickness $h_{c}>0$ [Shaw and Lin, 1996]. (b) Model 2: Requires the thickness of the lower crustal channel to be greater than a critical value $\left(h_{c}>h_{\mathrm{o}}\right.$ ), which represents the length scale over which stresses can be transmitted through the channel. (c) Model 3: Requires stress accumulation in the crust $\left(\sigma_{c}\right)$ to be much greater than the stress accumulation in the mantle $\left(\sigma_{c}\right)$. The results of this study favor Model 3 for typical geologic conditions.

which deformation transitions from the mantle-dominated to the crust-dominated regime. We present three potential models to explain this transition and discuss how the results of this study can be used to distinguish between these models.

In the first model, the transition between mantle-dominated and crust-dominated deformation should be abrupt, occuring when $t_{c}$ reaches the value required for the lower-most crust to behave in a ductile manner (Fig. 3-10a). This model is analogous to the analytical prediction of Shaw and Lin [1996] shown in Fig. 3-6, and assumes that the mere presence of a ductile lower crust is sufficient to decouple the upper crust from the upper mantle. The numerical results presented in this study, however, predict that rift half-width decreases over a range of crustal thicknesses (Figs. 3-6 and 3-9), not at a single, critical value as predicted by this model.

The second model assumes that deformation will become crust-dominated only when the thickness of the ductile lower crustal channel, $h_{c}$, becomes larger than a critical length 
scale, $h_{0}$, over which deformation can be effectively transmitted through the channel (Fig. 3-10b). In this senario, the range of crustal thicknesses over which the transition occurs, should be constant for all geothermal gradients and crustal rheologies. Yet this is also inconsistent with the results of our numerical experiments, which show that the range of crustal thicknesses in the "transition zone" over which rift half-width decreases is a function of both the geothermal gradient and the crustal rheology (Fig. 3-9). It is possible that for the thermal and rheologic conditions examined here, the thickness of the ductile layer never reaches the critical value, $h_{\mathrm{o}}$, necessary for decoupling. However, under typical geologic conditions it appears that decoupling cannot be explained by a critical length scale of the ductile lower crustal channel.

In the final model, crust-dominated deformation will only occur when the crustal thickness is sufficiently large that the stress accumulation in the upper crust, $\sigma_{c}$, becomes much greater than the stress accumulation in the upper mantle, $\sigma_{m}$ (Fig. 3-10c). This model appears most consistent with our numerical results, which show that $\sigma_{c}$ is typically $\sim 10$ times greater than $\sigma_{m}$ before deformation becomes completely crust-dominated. If correct, this model implies that under most geologic conditions the brittle upper crust will not behave independently of a strong upper mantle, regardless of the thickness of the lower crustal channel. This model is also in agreement with the instability analysis of Zuber et al. [1986] and modeling by Braun and Beaumont [1989] who found that the presence of a strong "fibre" in the upper mantle could affect the necking style of extending lithosphere. Based on these results, we hypothesize that complete decoupling between the upper crust and a strong upper mantle may not occur in many extensional systems.

\subsubsection{Comparison to Natural Rifts}

A common approach that has been used to explain the development of wide versus narrow rift zones is to integrate the strength of the lithosphere with depth and estimate the force needed for continued extension [e.g., Buck, 1991; England, 1983; Sonder and England, 1989]. If the force decreases with time, extension should stay localized and a narrow rift 
will form. Alternatively, if the force increases then extension will migrate laterally forming a wide rift. The effects of thermal structure, strain-rate, crustal thickness, and lower crustal flow can be incorporated into these models, and the sensitivity of rift style on each of parameter can be assessed [Buck, 1991].

However, while these 1-D models are useful for examining the long-term evolution of extensional systems, they cannot predict the specific pattern of deformation that will be produced by a given set of thermal and rheological conditions. In contrast, the numerical approach presented here allows us to explicitly calculate the preferred location of fault formation for a given set of initial conditions. Fig. 3-5a illustrates how wide rift zones can develop under laterally homogeneous thermal conditions. In contrast, when a regional temperature gradient is present, extension will focus onto one or two primary normal faults and a narrow rift will form (Fig. 3-5d). The width of narrow rifts is highly dependent on the specific horizontal thermal conditions of the region. However, if we assume that the bounding faults of most narrow rifts are controlled by a relatively broad thermal gradient, we can test the predictions of our numerical models with observations from natural extensional systems.

Table 3.3 provides a summary of crustal thickness, heat flow, and rift width for both narrow continental and oceanic rift zones. Corresponding geothermal gradients for continental rifts have been calculated from heat flow values assuming [Pollack and Chapman, 1977]

$$
T=T_{\mathrm{o}}+\frac{\left(q_{\mathrm{o}}-q_{m}\right) d}{K}\left(1-\exp \left(\frac{-z}{d}\right)\right)+\frac{q_{m}}{K} z
$$

where $T_{\mathrm{o}}$ is the surface temperature, $q_{\mathrm{o}}$ is the surface heat flow, $q_{m}$ is the mantle (or reduced) heat flow, $d$ is a characteristic depth scale, $K$ is the thermal conductivity, and $z$ is depth. We assume $T_{\mathrm{o}}=10^{\circ} \mathrm{C}, d=10 \mathrm{~km}, K=3.35 \mathrm{Wm}^{-1}{ }^{\circ} \mathrm{K}^{-1}$, and $q_{m}$ to be $60 \%$ of $q_{\mathrm{o}}$ for all calculations. Because hydrothermal circulation makes heat flow measurements at mid-ocean ridges difficult to interpret, geothermal gradients at the Mid-Atlantic Ridge and 


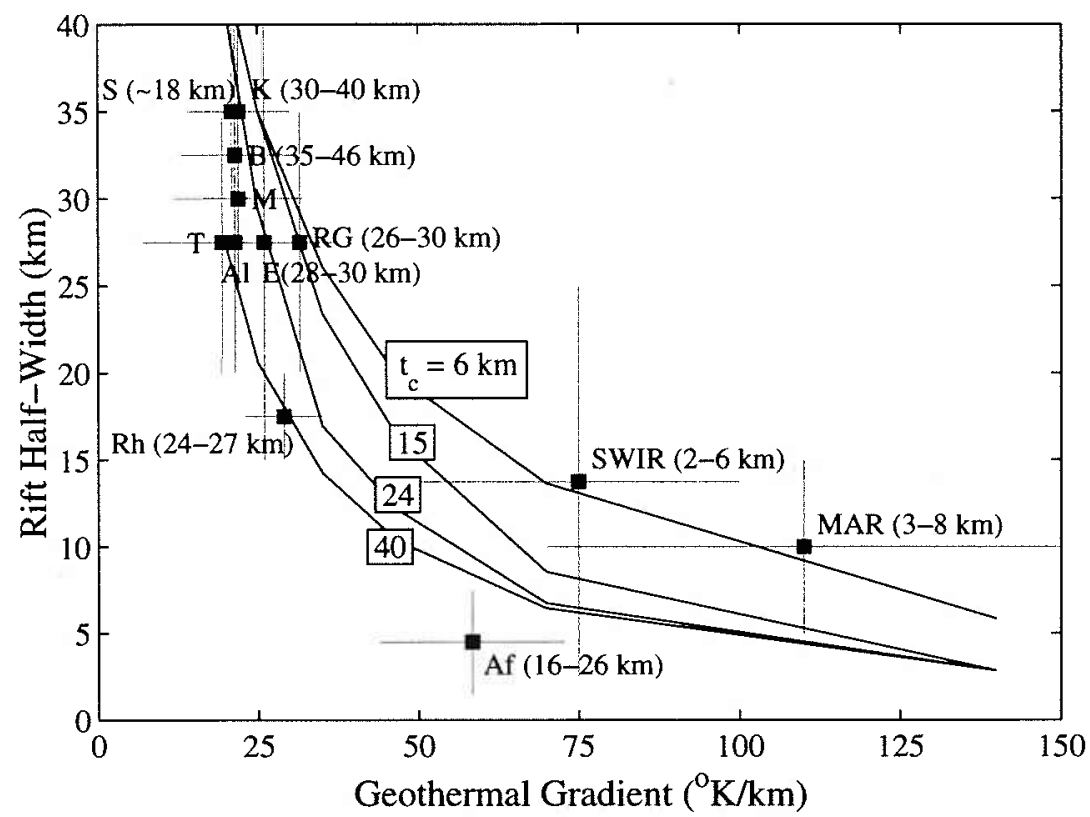

Figure 3-11: Predicted rift half-width as a function of geothermal gradient for values of $t_{c}$ $=6,15,24,40 \mathrm{~km}$ (solid lines). Solid squares show data from the narrow rifts compiled in Table 3 with observed crustal thickness given in parentheses. Error bars for data points are shown with grey lines.

Southwest Indian Ridge were calculated from the passive flow model of Phipps Morgan and Forsyth [1988].

In Fig. 3-11 we compare model predictions of rift half-width with the data for the natural rift zones compiled in Table 3.3. Predicted rift half-widths are calculated as a function of geothermal gradient for an intermediate crustal rheology and crustal thickness values of $6,15,24$, and $40 \mathrm{~km}$, respectively. Although there is a good deal of uncertainty involved in estimating geothermal gradients for natural rift zones, the observed rift halfwidths decrease with increasing geothermal gradient, which is in general agreement with the predictions of our model. Future work will involve more focused studies of individual rifting envirnoments in order to place more quantitative constraints on the thermal and rheologic conditions at depth. 
Table 3.3: Geophysical Parameters for Narrow Rift Zones

\begin{tabular}{|c|c|c|c|c|}
\hline Rift Zone & $\begin{array}{l}\text { Rift Width } \\
(\mathrm{km})\end{array}$ & $\begin{array}{c}t_{c} \\
(\mathrm{~km})\end{array}$ & $\begin{array}{l}\text { Heat Flow } \\
\left(\mathrm{mW} / \mathrm{m}^{2}\right)\end{array}$ & $\begin{array}{c}\frac{\partial T}{\partial z} \\
\left({ }^{\circ} \mathrm{K} / \mathrm{km}\right)\end{array}$ \\
\hline Suez Rift $(S)^{1}$ & $60-80$ & $\sim 18$ & $57-86$ & $17-25$ \\
\hline Albert Rift (Al) $^{2}$ & $40-70$ & - & $28-118$ & $8-35$ \\
\hline Tanganyika Rift $(\mathrm{T})^{3}$ & $40-70$ & - & $25-110$ & $7-32$ \\
\hline Malawi Rift $(M)^{4}$ & 50 & - & $40-110$ & $12-32$ \\
\hline Baikal Rift (B) ${ }^{5}$ & $50-80$ & $35-46$ & $45-100$ & $13-30$ \\
\hline Kenya Rift $(K)^{6}$ & $60-80$ & $30-40$ & $50-100$ & $14-30$ \\
\hline Ethiopian Rift $(E)^{7}$ & $30-80$ & $28-30$ & $75-100$ & $22-30$ \\
\hline Rhine Graben $(\mathrm{Rh})^{8}$ & $30-40$ & $24-27$ & $80-120$ & $23-35$ \\
\hline Rio Grande Rift (RG) ${ }^{9}$ & $\sim 50$ & $26-30$ & $90-125$ & $26-37$ \\
\hline Afar Rift (Af) ${ }^{10}$ & $3-15$ & $16-26$ & $150-250$ & $44-73$ \\
\hline SW Indian Ridge (SWIR) $)^{11}$ & $5-50$ & $2-6$ & N.A. & $50-100$ \\
\hline Mid-Atlantic Ridge (MAR) ${ }^{12}$ & $10-30$ & $3-8$ & N.A. & $70-150$ \\
\hline
\end{tabular}

\footnotetext{
${ }^{1}$ Patton et al. [1994]; Moustafa [1996]; Gaulier et al. [1988]; Steckler et al. [1998]

${ }^{2}$ Ebinger [1989]; Upcott et al. [1996]; Degens et al. [1973]

${ }^{3}$ Ebinger [1989]; Burgess et al. [1988]; Degens et al. [1971]

${ }^{4}$ Ebinger et al. [1987]; Von Herzen and Vacquier [1967]

${ }^{5}$ Diament and Kogan [1990]; Hutchinson et al. [1992]; Logatchev and Zorin [1992]; Poort et al. [1998] Morgan [1982]; Lysak [1987, 1992]

${ }^{6}$ King [1978]; Ebinger et al. [1989]; Braile et al. [1994]; Maguire et al. [1994]; Wheildon et al. [1994]

${ }^{7}$ Hayward and Ebinger [1996]; Makris and Ginzburg [1987]; Ebinger and Ibrahim [1994]; Lysak [1987]

${ }^{8}$ Wenzel et al. [1991]; Gutscher [1995]; Wenzel et al. [1991]; Prodehl et al. [1992]; Mayer et al. [1997] Morgan [1982]; Cermak et al. [1991]

${ }^{9}$ Chapin and Cather [1994]; Sinno et al. [1986]; Keller et al. [1990]; Decker and Smithson [1975] Morgan [1982]

${ }^{10}$ Hayward and Ebinger [1996]; Makris and Ginzburg [1987]; Lysak [1987]

${ }^{11}$ Mendel et al. [1997]; Grindlay et al. [1998]; Minshull and White [1996]; Muller et al. [1999]

${ }^{12}$ Sempéré et al. [1993]; Tolstoy et al. [1993]
} 


\subsection{Conclusions}

The results of this study illustrate how strain-rate softening in the brittle regime affects the pattern of lithospheric deformation in extensional settings. For strain-rate softening coefficients $\geq 0.10$, localized zones of high strain-rate are predicted to develop in response to the rheology and boundary conditions. We argue that these narrow shear zones, typically characterized by strain rates 4-6 orders of magnitude greater than the adjacent regions, are analogous to normal faults. Because these fault-like zones are not imposed a priori, we have used the calculated deformation field to investigate the sensitivity of crustal deformation to thermal state, crustal thickness, and rock rheology.

When no regional temperature gradient is imposed, deformation is predicted to be distributed between several sets of conjugate normal faults. However, in the presence of a horizontally varying temperature field, faulting is predicted to focus where the lithosphere is thinnest. We also investigated the effect of crustal thickness, crustal rheology, and geothermal gradient on rift half-width. Our numerical results predict that when crustal thickness is small, deformation will be mantle-dominated and rift half-width will be controlled primarily by the geothermal gradient. In contrast, when crustal thickness is large and deformation becomes crust-dominated, rift half-width will be a function of both crustal rheology and the geothermal gradient.

We hypothesize that the transition between crust-dominated and mantle-dominated deformation is related to the relative stress accumulation in the upper crust and upper mantle. Model results predict that for the range of parameters examined in this study, crustdominated deformation will occur only when the stress accumulation in the upper crust becomes much greater than the stress accumulation in the upper mantle. This implies that under typical geologic conditions the brittle upper crust does not behave independently of a strong upper mantle, regardless of the thickness of the ductile lower crustal channel. Consequently, complete decoupling of the upper crust and upper mantle may not occur in many extensional systems. 


\title{
Chapter 4
}

\section{Mechanisms for normal fault}

\section{development at mid-ocean ridges*}

\begin{abstract}
Slow-spreading ridge segments are characterized not only by small, closely spaced faults that develop near the segment center, but also by large, widely spaced faults that develop near the segment ends, typically at the inside corner of a ridge-offset intersection. In this study we investigate the competing effects of stress accumulation in the lithosphere and the yield strength of the lithosphere in controlling the location of normal fault formation and direction of propagation. Seismic velocity models from the Mid-Atlantic Ridge in the Oceanographer-Hayes region and $29^{\circ} \mathrm{N}$ and the East Pacific Rise at $9^{\circ} \mathrm{N}$ were used to estimate the along-axis change in dynamic Young's modulus. Corresponding thermal and rheologic models were calculated to estimate the along-axis variation in yield strength. We then develop a thin-plate model to calculate the predicted location of fault initiation or reactivation and the subsequent propagation direction for different combinations of linear along-axis gradients in Young's modulus and yield strength. Based on this model we define two modes of normal fault development at slow-spreading segments: Mode C (Center) faults, which develop at the segment center and propagate outward, and Mode E (End) faults, which develop at the segment ends and propagate inward. Mode $\mathrm{C}$ faults are predicted to form at ridges where the along-axis variation in yield strength dominates the along-axis accumulation of stress. Conversely, Mode E faults are predicted to develop at ridges where stress accumulation toward segment ends overcomes the high yield strength in these locations. In addition to the accumulation of stress caused by along-axis gradients
\end{abstract}

${ }^{*}$ In press: M.D. Behn, J Lin, and M.T. Zuber, Mechanisms for normal fault development at mid-ocean ridges, J. Geophys. Res., v. 107, 2002 
in Young's modulus, we illustrate that shear stresses resisting relative plate motion along a transform fault will generate higher effective stress at inside corners, possibly concentrating Mode E faulting in these locations. At fast-spreading ridges, where along-axis gradients in stress and lithospheric strength are relatively small, more uniform patterns of faulting are predicted. The results of this study quantify how the interplay between the along-axis variations in stress state and the mechanical properties of the lithosphere controls the style of fault development at mid-ocean ridge segments.

\subsection{Introduction}

The structure of oceanic crust formed at a ridge axis is controlled by the interplay between magmatic and tectonic processes. To first order, the relative importance of tectonic extension by faulting in seafloor spreading appears to increase with decreasing spreading rate [Edwards et al., 1991; Carbotte and Macdonald, 1994; Goff et al., 1997]. Furthermore, the style of tectonic faulting also varies with spreading rate. Faults formed at slow-spreading ridges are generally longer, with greater throw and larger spacing than faults formed at faster spreading rates [e.g., Searle and Laughton, 1981; Macdonald, 1982]. Systematic variations in faulting are also observed along individual segments of the slow-spreading Mid-Atlantic Ridge (MAR) [e.g., Shaw, 1992; Shaw and Lin, 1993; Escartín et al., 1999]. Near segment centers, where negative residual gravity anomalies [e.g., Kuo and Forsyth, 1988; Lin et al., 1990; Detrick et al., 1995] and seismic velocity structure [e.g., Tolstoy et al., 1993; Hooft et al., 2000] indicate crustal thickness to be greatest, faults are observed to be linear, closely spaced, with relatively small throws. Toward segment offsets, however, faults become more oblique, fault spacing increases, and the amount of throw on an individual fault is observed to increase (see Figure 4-1) [e.g., Shaw and Lin, 1993; Searle et al., 1998; Escartín et al., 1999]. In Figure 4-2 we present a geologic map of the MAR segment at $25^{\circ} 10^{\prime} \mathrm{N}$ (Segment 6 of Purdy et al. [1990]) based on a combination of multibeam bathymetry data [Purdy et al., 1990; Smith et al., 1995] and Towed Ocean Bottom Instrument (TOBI) side-scan sonar data [Smith et al., 1995]. Fault throw was mapped along individual faults based on strike-perpendicular bathymetry profiles, spaced every 1 


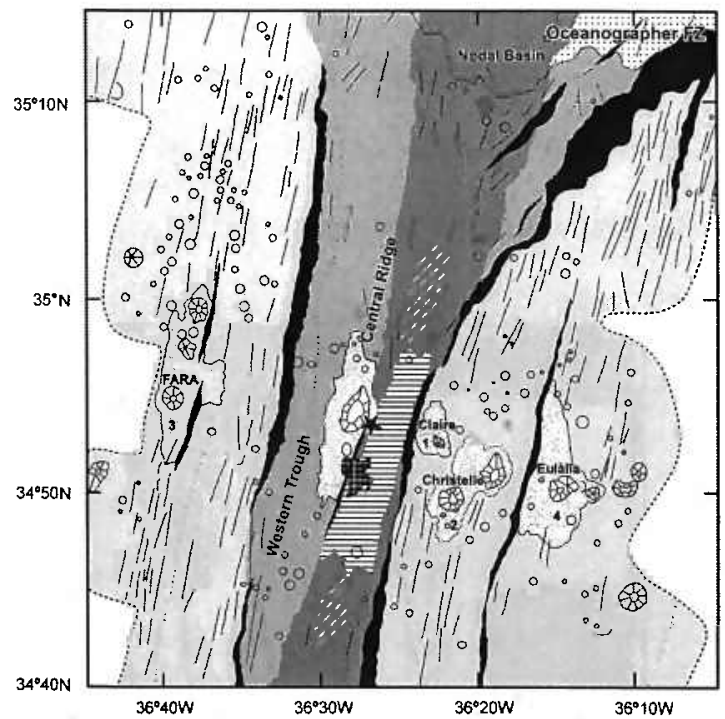

a) Segment $\mathrm{OH} 1$

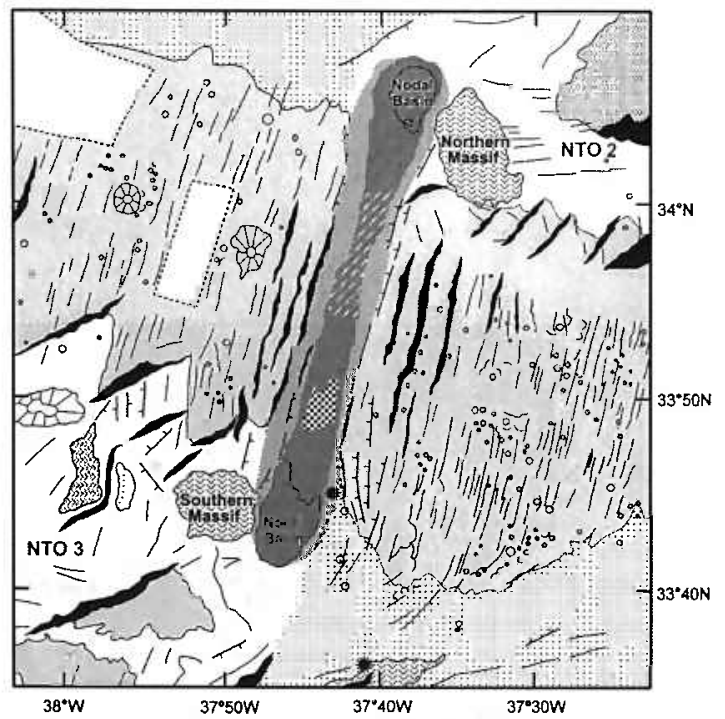

b) Segment $\mathrm{OH} 3$

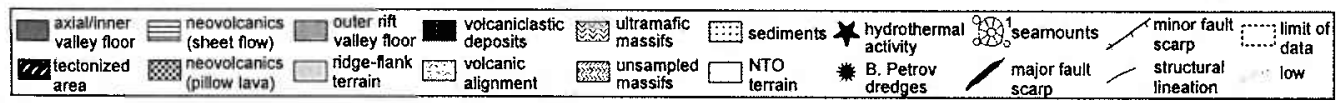

Figure 4-1: Geologic maps from Gràcia et al. [1999] of (a) MAR segment OH-1 and (b) segment $\mathrm{OH}-3$ based on a combination of bathymetry, acoustic backscattering, submersible observations, and rock samples. Major fault scarps are shown in black. At both segments, faults appear to develop not only near segment center (Mode C faults), but also toward segment ends (Mode $\mathrm{E}$ faults). Note the asymmetry in faulting toward the segment ends, with most major faults forming on the inside-corner crust near the Oceanographer fracture zone (in a) and non-transform offsets NTO2 and NTO3 (in b). Figure from Gràcia et al. [1999].

$\mathrm{km}$. Stars mark the locations of maximum throw along those faults for which it was possible to confidently determine throw along the entire length of the fault scarp.

Malinverno and Cowie [1993] and Shaw and Lin [1996] attributed the first-order dependence of fault style on spreading rate to changes in the mechanical strength of the lithosphere caused by the difference in thermal state between fast- and slow-spreading ridges. Moreover, the observed change in fault throw and spacing along individual slow-spreading segments has been hypothesized to reflect segment scale variations in the strength of the brittle lithosphere [Shaw, 1992; Shaw and Lin, 1996]. Near segment centers, where warmer temperatures and thicker crust are predicted, yield strength calculations show lithospheric strength to be significantly reduced relative to the segment ends [Shaw and Lin, 1996; Hirth et al., 1998]. 

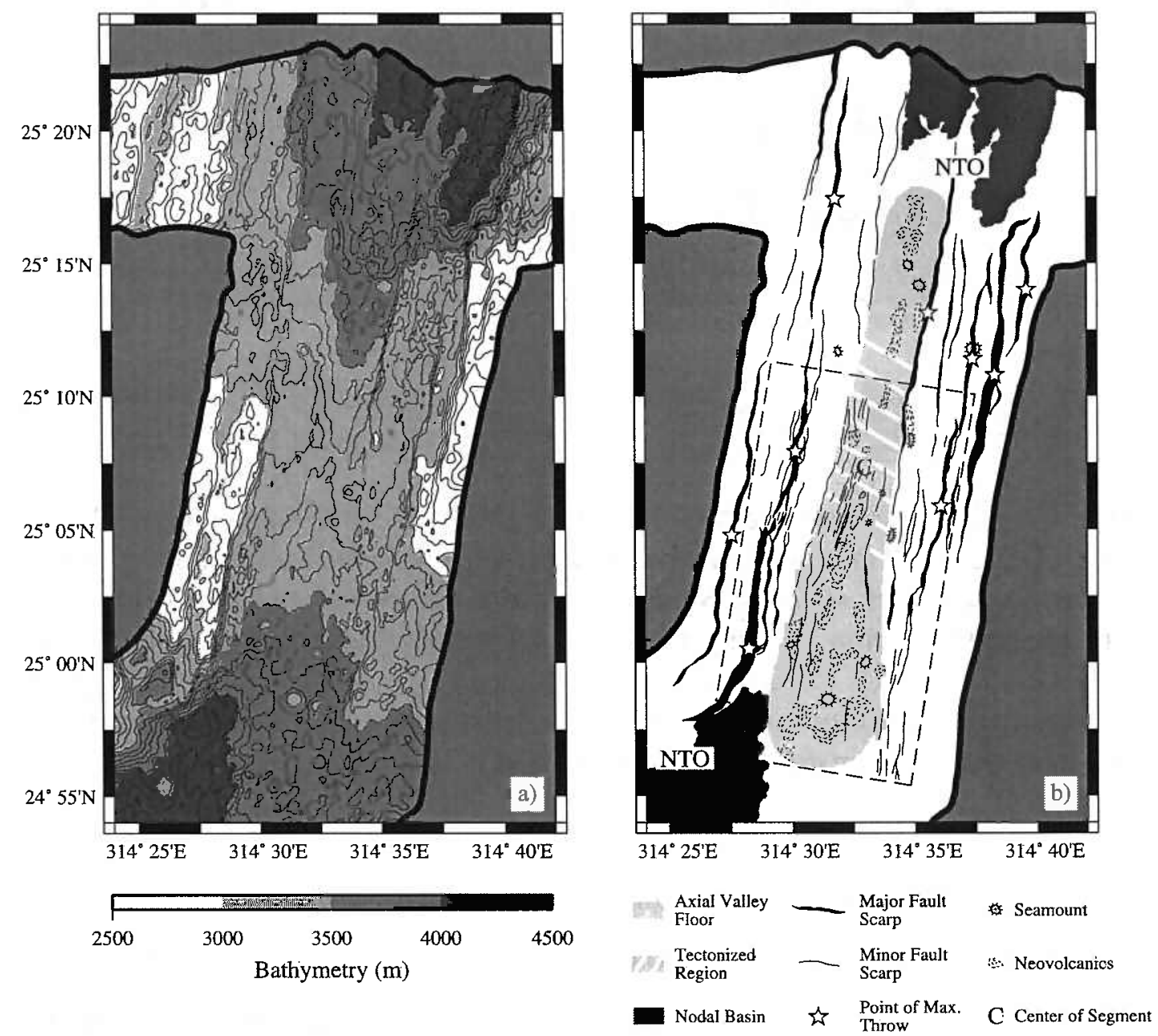

Figure 4-2: (a) Multi-beam bathymetry from Purdy et al. [1990] and Smith et al. [1995] for the MAR segment at $25^{\circ} 10^{\prime} \mathrm{N}$. Note the hour-glass shape morphology of the axial valley, which is characteristic of many magmatically robust slow-spreading segments. (b) Geologic map of the $25^{\circ} 10^{\prime} \mathrm{N}$ segment based on the multi-beam bathymetry data in (a) and TOBI side-scan sonar data from Smith et al. [1995]. Dashed line delineates the area within which the TOBI data are available. Throw of faults was measured along major fault scarps, and location of maximum throw is marked with a star. Note that major faults develop not only at the segment center (Mode C faults), but also near the segment ends (Mode E faults), particularly on inside-corner crust. 
The symmetry of faulting is also observed to vary along individual slow-spreading segments. At the segment center faulting is generally symmetric across-axis, often with several inward dipping faults nested to form the median valley [e.g., Macdonald, 1982]. Toward the segment ends, however, the crust becomes highly asymmetric with large throw, widelyspaced faults concentrated on the elevated crust of the inside corner of a ridge-transform intersection, and smaller more closely-spaced faults at the outside corner [Severinghaus and Macdonald, 1988; Tucholke and Lin, 1994; Allerton et al., 1995; Escartín et al., 1999]. Many of the faults formed on inside-corner crust are observed to have their maximum throw at or near the segment end (Figure 4-2). This observation suggests that these faults have initiated, or are preferentially reactivated, in these locations rather than at the segment center.

These observations raise a fundamental question regarding the mechanics of faulting at mid-ocean ridges. Namely, if thicker crust and elevated temperatures indicate a weaker plate at segment centers, why are many faults observed to initiate, or preferentially reactivate, in the stronger lithosphere near segment ends, particularly on inside-corner crust? One potential explanation is that at certain times stress accumulation in the lithosphere is enhanced toward the segment ends. Such an along-axis gradient in stress could be achieved if stress is preferentially released near the segment center due to greater magmatic extension in these areas [Karson and Winters, 1992; Gràcia et al., 1999], while continuing to accumulate at the segment ends. Alternatively, variations in the elastic properties of the lithosphere [e.g., Campbell, 1978] or the geometry of a ridge-transform intersection [e.g., Phipps Morgan and Parmentier, 1984; Pollard and Aydin, 1984; Grindlay and Fox, 1993] could generate higher stresses toward segment ends.

Here we present the results of a modeling study to quantify the competing effects of stress accumulation in the lithosphere and the mechanical strength of the brittle plate, in order to predict the style of normal fault development and propagation. We show that at fast-spreading ridges the lack of strong along-axis gradients in stress and lithospheric strength lead to relatively uniform fault patterns. In contrast, at slow-spreading segments 
significant along-axis variations in these two parameters generate a more complex pattern of faulting depending on the relative importance of stress and the mechanical properties of the plate. These and other predictions are compared with observations, and the implications for different styles of fault development at fast- and slow-spreading ridges are discussed.

\subsection{Rheology of a Mid-Ocean Ridge Spreading Segment}

In order to understand how the state of stress and the mechanical strength of the lithosphere vary at a typical spreading segment, we first examine the constraints on these parameters at mid-ocean ridge spreading centers.

\subsubsection{Young's Modulus and State of Stress}

Stress accumulation in the lithosphere is influenced by the elastic properties of the brittle layer. Hooke's law dictates that stress in an elastic body is directly proportional to the strain applied on the body and the Young's modulus of material. Therefore, bodies with a greater Young's modulus will experience higher stress for a given strain than bodies with a lower modulus. Numerous laboratory studies have estimated the static elastic properties of rocks typically found on mid-ocean ridges [e.g., Bass, 1995, and refs. therein]. However, because laboratory experiments can only be performed on small rock samples, they are highly dependent on local heterogeneities in the sample. In order to avoid this problem, we take advantage of the fact that seismic velocity is a function of the elastic properties of the medium, and use P-wave velocity models to estimate the dynamic Young's modulus of the lithosphere [e.g., Cheng and Johnston, 1981; Eissa and Kazi, 1988]. The relationship between static and dynamic Young's modulus is somewhat complex, particularly at low confining pressures. However, laboratory and in-situ studies have found that in general the ratio of static to dynamic Young's modulus is between 1 and 2 [Eissa and Kazi, 1988; Gudmundsson, 1988; Forslund and Gudmundsson, 1991]. 
Rewriting the equation for P-wave velocity gives the following expression for dynamic Young's modulus [Jaeger and Cook, 1979]

$$
\mathrm{E}=\frac{\mathrm{v}_{\mathrm{p}}^{2}(1+\nu)(1-2 \nu) \rho}{(1-\nu)}
$$

where $\mathrm{v}_{\mathrm{p}}^{2}$ is the P-wave velocity, $\nu$ is the Poisson's ratio, and $\rho$ is the density. Applying Equation 4.1 to the P-wave velocity model of Canales et al. [2000b] for the western rift mountains of MAR segment $\mathrm{OH}-1\left(35^{\circ} \mathrm{N}\right)$, we calculated a corresponding Young's modulus model (Figure 4-3). The crustal and mantle densities were taken from the model of Canales et al. [2000b], which is based on the velocity-to-density relationships of Horen et al. [1996] and Miller and Christensen [1997] and constrained by the observed gravity. Estimates of Poisson's ratio in oceanic crust range from 0.25-0.32 [e.g., Christensen and Smewing, 1981; Bratt and Solomon, 1984; Collier and Singh, 1998]. We choose an average value of 0.30 for Poisson's ratio and assign an uncertainty of $\pm 15 \%$ to our calculations of Young's modulus.

In order to incorporate the observed variations in Young's modulus into a thin-plate model of a ridge segment, we assume that at any point the plate behaves in relation to the depth-averaged value of its rheologic properties. The dashed line in Figure 4-4a illustrates Young's modulus averaged to a depth of $8 \mathrm{~km}$, which is the maximum depth of reasonable seismic resolution, along the Canales et al. [2000b] profile shown in Figure 4-3b. The depth-averaged value of Young's modulus increases from segment center toward the distal ends with an approximate gradient of $0.15-0.25 \mathrm{GPa} / \mathrm{km}$, and then decreases by $\sim 10 \mathrm{GPa}$ within the offsets bounding the segment to the north and south. We performed a similar calculation along the axis of segment OH-1 using the P-wave velocity model of Hooft et al. [2000] (solid line in Figure 4-4a). This calculation suggests that while the along-axis gradient in depth-averaged Young's modulus is similar both on- and off-axis, the off-axis profile is shifted to higher moduli by a constant value of 5-8 GPa. This would predict an across-axis gradient in Young's modulus of $\sim 0.15 \mathrm{GPa} / \mathrm{km}$. 

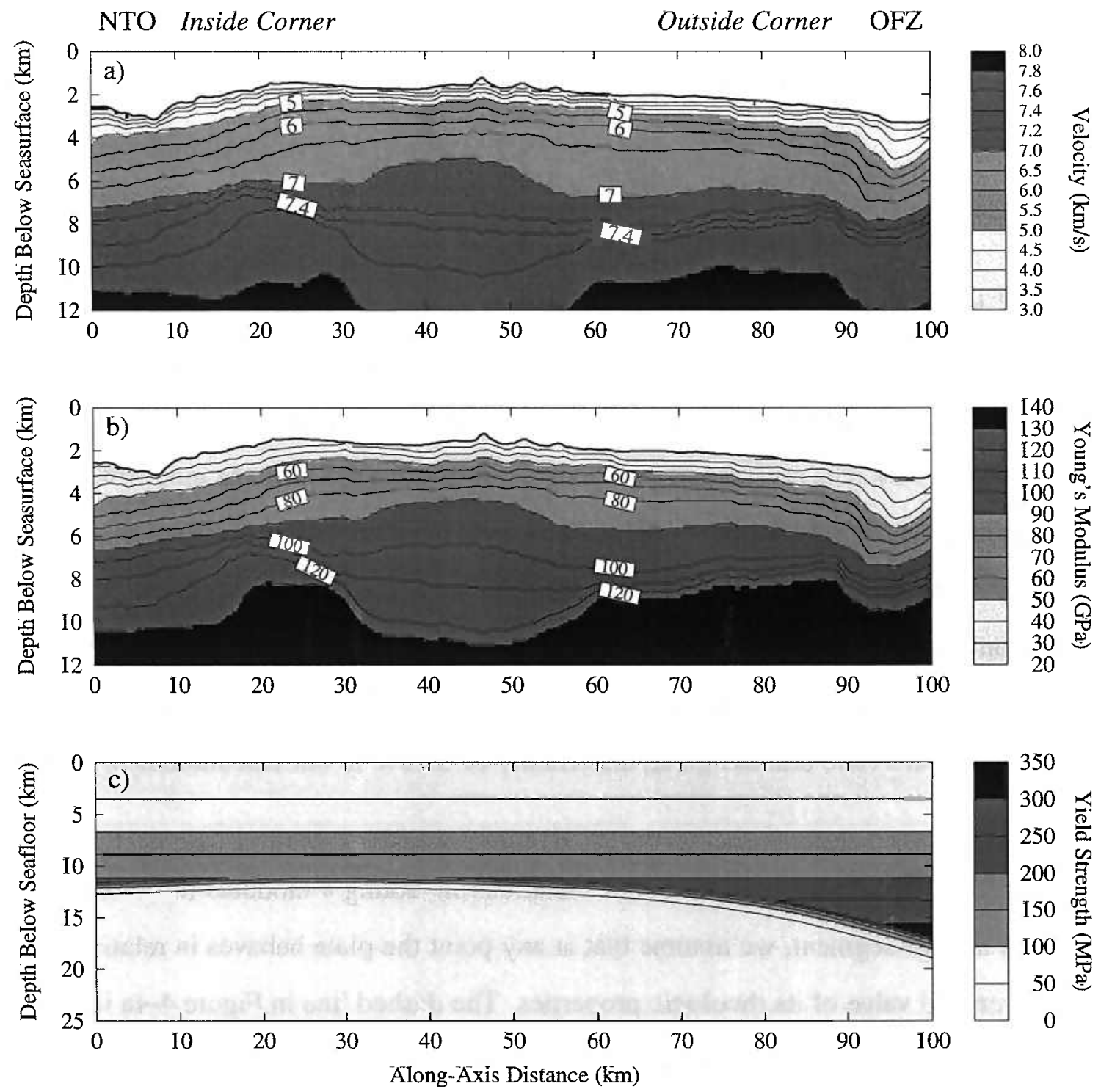

Figure 4-3: (a) Seismic P-wave velocity model of Canales et al. [2000b] along the western rift mountains of MAR segment OH-1 (see Figure 4-4 for location). Segment is bounded to the south by a non-transform offset and to the north by the Oceanographer fracture zone. (b) Calculated dynamic Young's modulus with depth along the OH-1 segment, based on the Pwave velocity and density models of Canales et al. [2000b] and assuming a Poisson's ratio of 0.30. See text for description of dynamic Young's modulus calculation. (c) Calculated yield strength depth section along the OH-1 segment. Yield strength is based on Byerlee's rule with a constant coefficient of friction $\mu=0.85$, above the brittle/ductile transition and the ductile flow law below the brittle/ductile transition. We assume the dry diabase flow law of Mackwell et al. [1998] for the crust and Chopra and Paterson [1984] dry dunite flow law for the mantle. The thermal structure for the $\mathrm{OH}-1$ segment was calculated using Phipps Morgan and Forsyth [1988] passive flow model with a half rate of $1.1 \mathrm{~cm} / \mathrm{yr}$ and a mantle temperature of $1350^{\circ} \mathrm{C}$ at a depth of $100 \mathrm{~km}$. 
We also calculated depth-averaged Young's modulus along the MAR segment at $29^{\circ} \mathrm{N}$ (Figure 4-4d) and the EPR segment at $9^{\circ} \mathrm{N}$ (Figure 4-4g) using the seismic velocity models of Wolfe et al. [1995] and Canales et al. [2001], respectively. The $29^{\circ} \mathrm{N}$ segment of the MAR shows a similar gradient to that of the OH-1 segment, while the EPR segment shows little along-axis variation in Young's modulus outside the fracture zone and overlapping spreading center at the segment ends. These results imply that the increase in depth-averaged Young's modulus toward the ends of a slow-spreading segment is caused by thinner crust in these locations, resulting in a greater portion of the brittle plate to be composed of high Young's modulus mantle rocks, such as olivine and pyroxene. In contrast, at fast-spreading ridges the observed variations are much smaller except locally near major ridge offsets.

An alternative approach is to average Young's modulus to the depth of the brittle-ductile transition, as opposed to a constant depth along the entire segment. The effect of this calculation would be to average to greater depths at the segment ends where the brittleductile transition is deeper (see Figure 4-3c), incorporating more high Young's modulus rocks of the lower crust and mantle and increasing the along-axis gradient. This approach was used along the OH-1 segment, and the resulting gradient was calculated to be $\sim 0.5$ $\mathrm{GPa} / \mathrm{km}$, approximately twice the value calculated when averaging to a constant depth of $8 \mathrm{~km}$. Thus, it is possible that the depth-averaged values shown in Figures 4-4a, 4-4d, and $4-4 \mathrm{~g}$ underestimate the true along-axis gradients in Young's modulus.

\subsubsection{Yield Strength}

The mechanical strength of the lithosphere is often modeled using a strength-versus-depth profile ("yield strength envelope"), in which strength in the shallow, brittle regime is controlled by a frictional resistance law [e.g., Byerlee, 1978], while strength in the deeper, ductile regime is limited by power law creep [e.g., Goetze and Evans, 1979; Brace and Kohlstedt, 1980] (Figure 4-5). 
Figure 4-4: (facing page) (a) Along-axis variation in the calculated Young's modulus averaged to a depth of $8 \mathrm{~km}$ based on the seismic refraction profiles of Canales et al. [2000b] (dashed) and Hooft et al. [2000] (solid). (b) Location of Canales et al. [2000b] and Hooft et al. [2000] seismic lines. (c) Along-axis variation in the calculated yield strength averaged to a depth of $15 \mathrm{~km}$ along the Canales et al. [2000b] and Hooft et al. [2000] seismic lines. See Figure 4-3 for description of parameters used in calculation of yield strength. Gray bars illustrate the location of the non-transform offset (NTO) and Oceanographer fracture zone (OFZ) bounding the OH-1 segment to the south and north, respectively. Small arrow shows segment mid-point. (d,e,f) Calculated Young's modulus, location, and yield strength, respectively, for two profiles through the 3-D tomographic P-wave inversion of Wolfe et al. [1995] at $29^{\circ} \mathrm{N}$ on the MAR. (g,h,i) Calculated Young's modulus, location, and yield strength, respectively, for two seismic refraction profiles Canales et al. [2001] on $\sim 300$ k.y.-old crust at $9^{\circ} \mathrm{N}$ along the EPR. Note that the two MAR segments display significant along-axis gradients in both Young's modulus $(0.2-0.3 \mathrm{GPa} / \mathrm{km})$ and yield strength $(0.1-1.0 \mathrm{MPa} / \mathrm{km})$, with the lowest values found at the center of the segment. In contrast, the EPR segment shows little variation in either Young's modulus or yield strength alongaxis, except within the Siqueiros fracture zone (SFZ) and overlapping spreading center (OSC) at the segment ends.

Depth-averaged yield strength was calculated along the OH-1, MAR $29^{\circ} \mathrm{N}$, and EPR $9^{\circ} \mathrm{N}$ segments. Using the technique of Phipps Morgan and Forsyth [1988], we consider conductive and advective heat transfer in mantle flow driven solely by separating surface plates. Thermal models were calculated for each of the three spreading segments based on the appropriate spreading rate and ridge-offset geometry. Along each of the available seismic lines, strength-versus-depth profiles were computed (e.g., Figure 4-3c) and averaged to a depth of $15 \mathrm{~km}$, below which changes are negligible. The resulting depth-averaged yield strengths for the three segments are shown in Figure 4-4. Because a constant coefficient of friction, $\mu=0.85$, was used in all calculations, the along-axis gradients in depth-averaged yield strength are primarily thermally controlled.

Due to the thermal cooling effect of ridge offsets, along-axis changes in depth-averaged yield strength are highly dependent on the length of the bounding offset. At MAR $29^{\circ} \mathrm{N}$, for example, where the offsets at either end of the segment are approximately $15 \mathrm{~km}$ in length, the predicted along-axis gradient in yield strength is $\sim 0.3 \mathrm{MPa} / \mathrm{km}$. In contrast, at the MAR OH-1 segment, where the northern end of the segment is bounded by the 

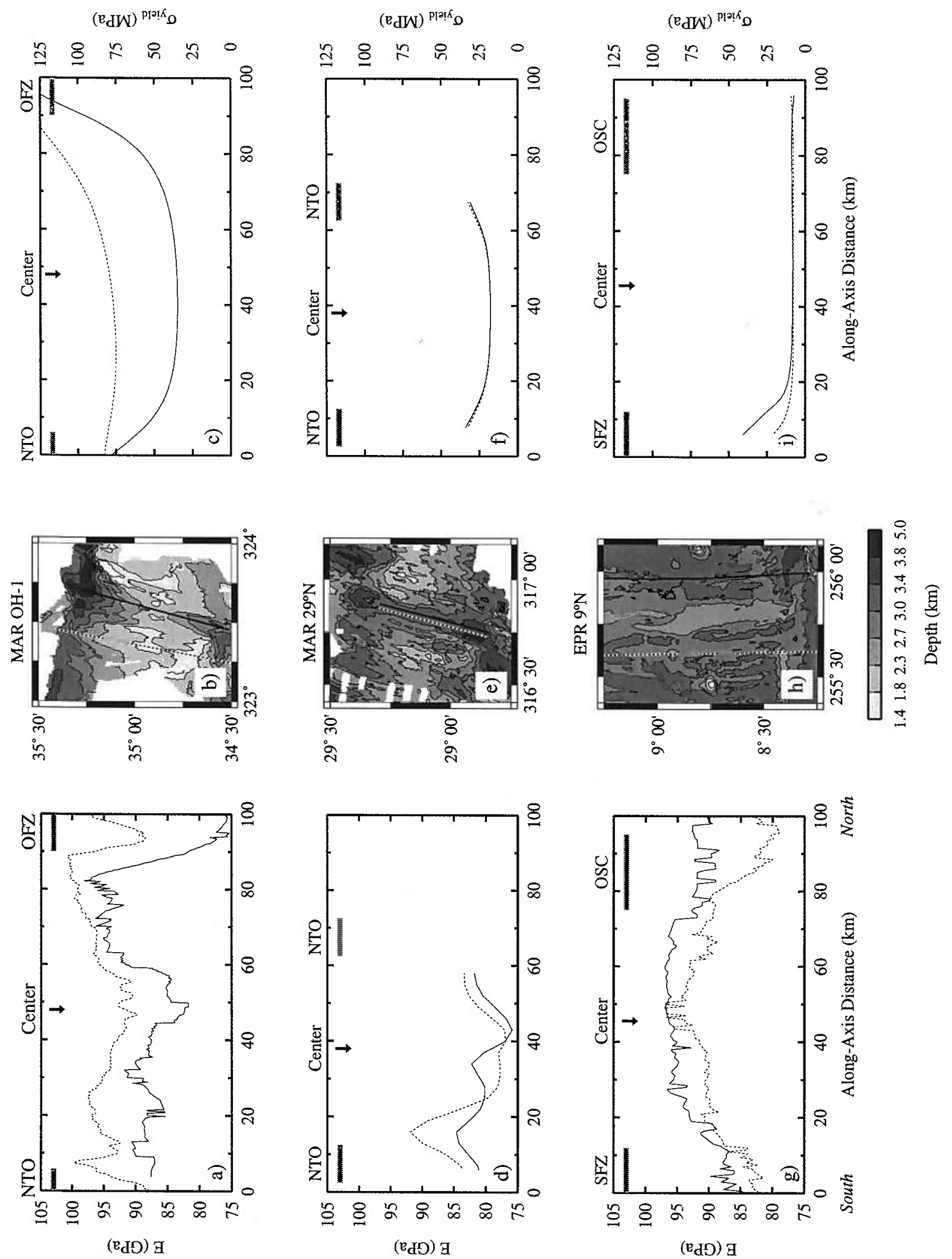

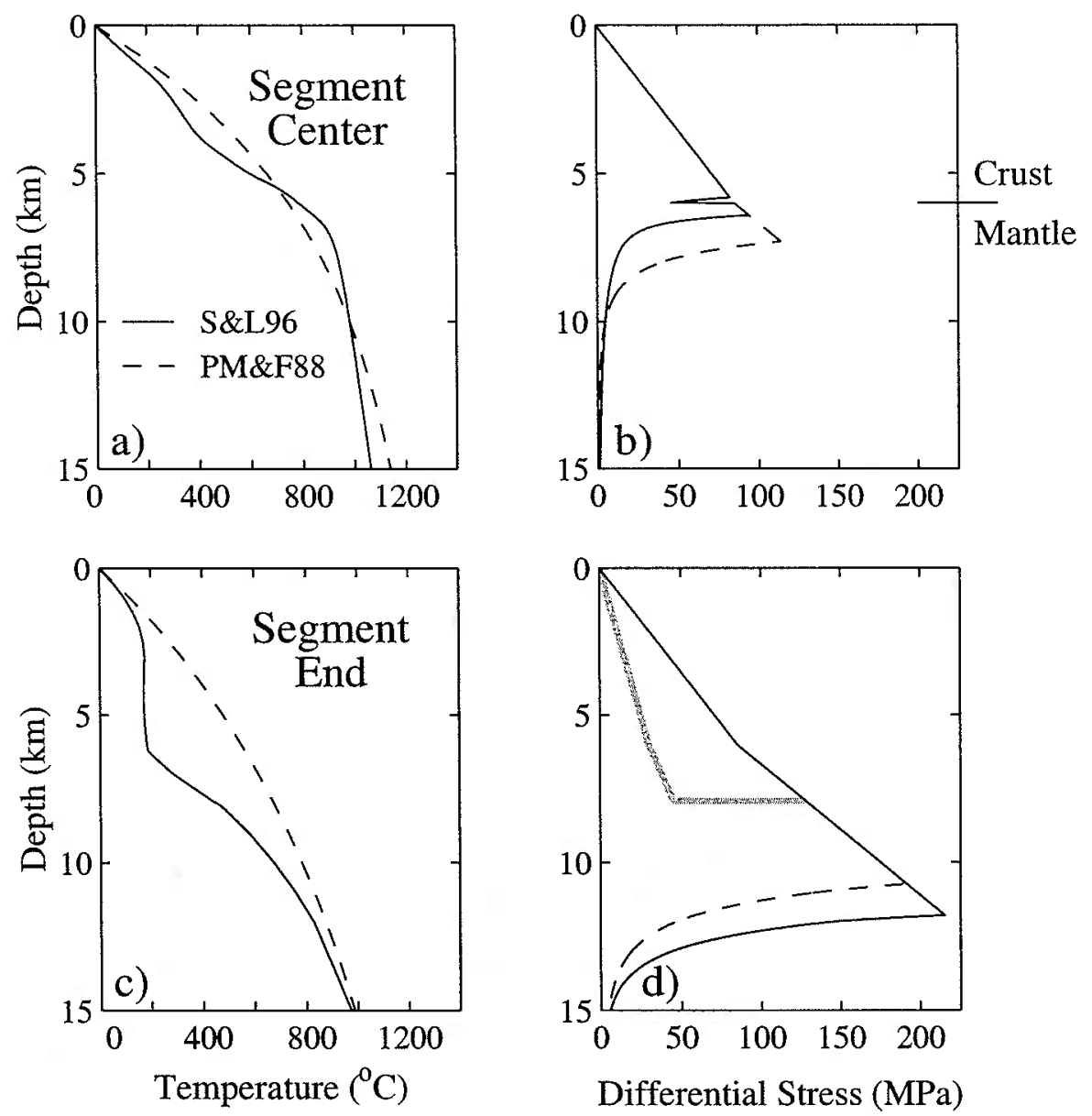

Figure 4-5: (a) Two temperature-depth profiles calculated at the center of a 50-km-long northern MAR segment. Dashed line (PM\&F88) shows temperatures calculated from the passive flow model of Phipps Morgan and Forsyth [1988], assuming a half-rate of $1 \mathrm{~cm} / \mathrm{yr}$ and a mantle temperature of $1350^{\circ} \mathrm{C}$ at a depth of $100 \mathrm{~km}$. Solid line (S\&L96) illustrates the NMARL thermal model of Shaw and Lin [1996] for similar parameters, but incorporates the effects of hydrothermal cooling in the crust and the heat of magma emplacement with variable crustal thickness. (b) Yield strength envelopes computed from thermal models in (a). Yield strength is calculated using Byerlee's rule with a constant coefficient of friction, $\mu=0.85$, above the brittle/ductile transition and the ductile flow law below the brittle/ductile transition. We assume the dry diabase flow law of Mackwell et al. [1998] for the crust and Chopra and Paterson's [1984] dry dunite flow law for the mantle. A strain rate of $10^{-14} \mathrm{~s}^{-1}$ was used for all calculations. (c,d) Thermal profiles and corresponding yield strength envelopes calculated at the end of the NMARL segment. Thick gray line illustrates the predicted effect of serpentinization on the brittle regime above the $400^{\circ} \mathrm{C}$ isotherm when using the coefficient of friction for serpentinite $(\mu=0.3)$ from Escartin et al. [1997]. Note that the Shaw and Lin [1996] thermal model predicts lower strength at the segment center and greater strength at the segment ends relative to the model of Phipps Morgan and Forsyth [1988]. 
$100 \mathrm{~km}$ Oceanographer fracture zone, the predicted along-axis gradient in yield strength is $>2.0 \mathrm{MPa} / \mathrm{km}$. The along-axis variation in yield strength is also strongly dependent on spreading rate, with higher rates having smaller along-axis changes. Note that even though the southern end of the EPR $9^{\circ} \mathrm{N}$ segment is bounded by the $75 \mathrm{~km}$ long Siqueiros fracture zone, the depth-averaged yield strength remains relatively constant up to distances of $<10$ $\mathrm{km}$ from the offset.

The Phipps Morgan and Forsyth [1988] model neglects the effects of hydrothermal cooling in the shallow crust and the heat of magma emplacement at the ridge axis, which were considered in the models of Shaw and Lin [1996] (Figure 4-5a). Hydrothermal circulation preferentially cools those portions of the lithosphere where open cracks permit fluid flow beneath the seafloor. Shaw and Lin [1996] suggest this process would be enhanced toward segment ends, intensifying the cooling effect of ridge offsets, and further increasing the mechanical strength of the lithosphere in these locations (Figure 4-5d). Magma injection, on the other hand, would tend to increase temperatures near the center of a slowspreading segment, where gravity and seismic models suggest greater crustal emplacement. This would decrease the mechanical strength of the lithosphere at the segment center (Figure 4-5b), and when combined with the effect of hydrothermal cooling, lead to stronger along-axis gradients in depth-averaged yield strength. We compare depth-averaged yield strength calculated at the center and end of a 50-km-long northern MAR segment using the Phipps Morgan and Forsyth [1988] and Shaw and Lin [1996] models. The results suggest that the addition of hydrothermal cooling and the heat of magma emplacement, as included in the Shaw and Lin [1996] model, may increase the along-axis gradient in yield strength by $\sim 50 \%$.

In contrast, the effect of serpentinization may partially offset these effects. Serpentinized peridotites are observed to outcrop preferentially toward the end of slow-spreading segments, in particular at the inside corner of a ridge-offset intersection [Karson et al., 1987; Tucholke and Lin, 1994; Cannat et al., 1995]. Escartín et al. [1997] observed that serpentinites have a low coefficient of friction, $\mu \sim 0.3$, and thus can reduce the integrated strength of the lithosphere up to $30 \%$ toward the segment ends (Figure 4-5d). Therefore, if 
the coefficient of friction decreases from the center to the ends of a spreading segment due to the effects of serpentinites, it may reduce the along-axis gradient in yield strength.

In summary, our calculations indicate that the along-axis gradients in depth-averaged Young's modulus and yield strength are in the range of $0.15-0.5 \mathrm{GPa} / \mathrm{km}$ and $0.2-2.5$ $\mathrm{MPa} / \mathrm{km}$, respectively, along the slow-spreading MAR. In contrast, at the fast-spreading EPR, there appears to be little variation in either of these two parameters along a spreading segment, with the possible exception of a narrow $(5-10 \mathrm{~km})$ zone adjacent to a major offset. Due to the uncertainties involved, we use these estimates only as qualitative limits on the variations along an individual spreading segment. We then parameterize a thin-plate deformation model using linear gradients in Young's modulus and yield strength from the segment center to the distal ends.

\subsection{Model Setup}

To date most studies of faulting at mid-ocean ridges have focused on deformation in crosssections through the lithosphere [e.g., Tapponnier and Francheteau, 1978; Phipps Morgan et al., 1987; Chen and Morgan, 1990; Lin and Parmentier, 1990; Shaw and Lin, 1996; Buck and Poliakov, 1998; Poliakov and Buck, 1998]. However, in this study we attempt to quantify the spatial pattern of normal fault development along a ridge segment. We construct a thin-plate model for a single ridge segment. A plane stress approximation is adopted, in which the vertical tectonic stresses are assumed to be negligible relative to the horizontal stresses in the lithosphere and there is no stress coupling between the lithosphere and its underlying ductile asthenosphere. As illustrated in Figure 4-4, both the depth-averaged Young's modulus and yield strength are expected to vary along midocean ridge segments, with the gradient a function of spreading rate and other factors. To parameterize the observed increase in Young's modulus, E, and yield strength, $\sigma_{\text {yield }}$, from segment center to the distal ends, we impose linear gradients in these two parameters $(\mathrm{dE} / \mathrm{dy}$ and $\mathrm{d} \sigma_{\text {yield }} / \mathrm{dy}$, respectively) along the ridge axis (Figures 4-6b and 4-6c). We also impose an across-axis gradient in yield strength $\left(\mathrm{d} \sigma_{\text {yield }} / \mathrm{dx}\right)$ twice the magnitude of the along-axis 
(a)

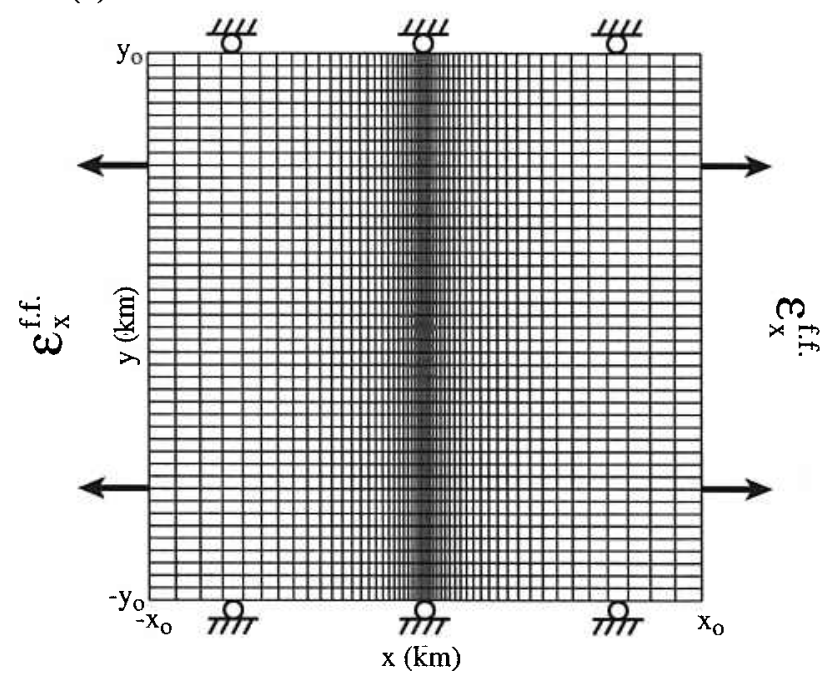

(b)

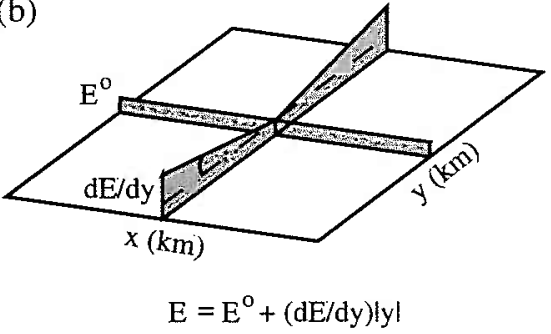

(c)

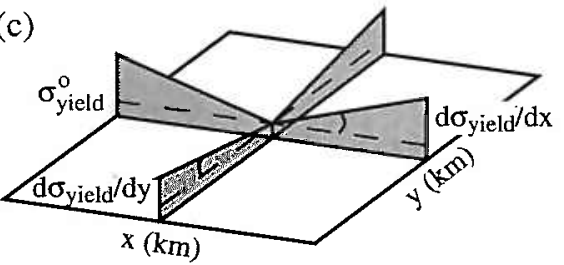

$\sigma_{\text {yield }}=\sigma_{\text {yield }}^{o}+\left(\mathrm{d} \sigma_{\text {yield }} / \mathrm{dx}\right)|\mathrm{x}|+\left(\mathrm{d} \sigma_{\text {yield }} / \mathrm{d} \mathrm{y}\right)|\mathrm{y}|$

Figure 4-6: (a) The lithosphere at a mid-ocean ridge segment is modeled as a thin plate in plane stress undergoing uni-axial extension. The model space is $100 \mathrm{~km} \mathrm{x} 100 \mathrm{~km}$, with finer grid spacing toward the ridge axis. The north and south edges of the model are assigned to be free-slip boundaries with no displacement in the y-direction. (b, c) Each element is assigned a Young's Modulus, E, and a yield strength value, $\sigma_{\text {yield }}$, which are allowed to vary linearly along- and across-axis.

gradient in order to account for the thermal thickening of the lithosphere with age [e.g., Watts et al., 1980] (see Table 4.1 for complete list of model parameters). Although Figure 4-4a suggests that an across-axis gradient in Young's modulus may be present at a typical slow-spreading ridge segment, our calculations show this gradient does not significantly influence the results of our model (see Appendix for complete discussion), and thus we assume Young's modulus to be constant in the across-axis direction (Figure 4-6b).

A gradually increasing extensional strain is applied at far-field model boundaries (left and right) to simulate spreading and the resulting stresses are calculated analytically throughout the model domain (see Appendix). The far-field strain, $\varepsilon_{x}^{f . f .}$, is increased until the effective stress in the plate, defined as $\sigma_{\text {eff }}=\left(\frac{1}{2}\left(\sigma_{\mathrm{x}}^{2}+\sigma_{\mathrm{y}}^{2}+\left(\sigma_{\mathrm{x}}-\sigma_{\mathrm{y}}\right)^{2}+6 \tau_{\mathrm{xy}}^{2}\right)\right)^{1 / 2}$, exceeds the material yield stress at some location in the model domain. Once yielding occurs the elastic-plastic finite element model, ADINA [Bathe, 1996], is used to calculate the stresses and strains numerically throughout the model domain. We assume that after yielding the 
Table 4.1: Model Paramters

\begin{tabular}{llll} 
& Meaning & Value & Units \\
\hline$\nu$ & Poisson's ratio & 0.3 & \\
$\rho$ & density & 0.85 & $\mathrm{~kg} / \mathrm{m}^{3}$ \\
$\mu$ & coefficient of friction & & $\mathrm{m} / \mathrm{s}$ \\
$\mathrm{v}_{\mathrm{p}}$ & p-wave velocity & 50 & $\mathrm{~km}$ \\
$\mathrm{x}_{\mathrm{o}}$ & across-axis model space half-width & 50 & $\mathrm{~km}$ \\
$\mathrm{y}_{\mathrm{o}}$ & along-axis model space half-width & $\Delta \mathrm{u}_{\mathrm{x}} / \mathrm{x}_{\mathrm{o}}$ & $\mathrm{km}$ \\
$\Delta \mathrm{u}_{\mathrm{x}}$ & far-field half-dispacement & & \\
$\varepsilon_{\mathrm{x}}^{\text {f.f. }}$ & far-field strain in x-direction & & $\mathrm{GPa}$ \\
$\varepsilon_{\Theta}^{\text {ff. }}$ yield & far-field strain at initial yielding & $\mathrm{GPa}$ \\
$\mathrm{E}$ & Young's modulus & $\mathrm{GPa} / \mathrm{km}$ \\
$\mathrm{E}^{\circ}$ & Young's modulus at segment center & $\mathrm{GPa} / \mathrm{km}$ \\
$\mathrm{dE} / \mathrm{dx}$ & across-axis gradient in Young's modulus & $\mathrm{MPa}$ \\
$\mathrm{dE} / \mathrm{dy}$ & along-axis gradient in Young's modulus & $\mathrm{MPa}$ \\
$\sigma_{\text {yield }}^{\circ}$ & yield strength & $2 * \mathrm{~d} \sigma_{\text {yield }} / \mathrm{dy}$ & $\mathrm{MPa} / \mathrm{km}$ \\
$\sigma_{\text {yield }}^{\circ}$ & yield strength at segment center & $\mathrm{MPa} / \mathrm{km}$ \\
$\mathrm{d} \sigma_{\text {yield }} / \mathrm{dx}$ & across-axis gradient in yield strength & $\mathrm{MPa}$ \\
$\mathrm{d} \sigma_{\mathrm{yield}} / \mathrm{dy}$ & along-axis gradient in yield strength & $\mathrm{MPa} / \mathrm{km}$ \\
$\sigma_{\text {eff }}$ & effective stress & $\mathrm{MPa}$ \\
$\mathrm{d} \sigma / \mathrm{dy}$ & additional gradient in $\sigma_{\text {eff to switch faulting modes }}$ & & $\mathrm{MPa}$ \\
$\sigma_{\mathrm{R}}$ & tensile stress resisting plate separation at ridge axis & $\sigma_{\text {yield }}^{\circ}$ & $\mathrm{MPa}$ \\
$\sigma_{\mathrm{T}}$ & shear stress resisting plate motion along offset & & \\
$\Delta \sigma_{\mathrm{RT}}$ & change in $\sigma_{\text {eff }}$ due to ridge-transform intersection & & $\mathrm{M}$ \\
\hline
\end{tabular}


plate behaves as a perfectly plastic material, and the evolution of the plastic yield zone is examined to provide a qualitative description of the resulting region of normal faulting at the ridge axis.

\subsection{Model Results}

As the far-field extensional strain increases, stress will accumulate throughout the model space, until the effective stress exceeds the yield stress and failure occurs. Because the yield strength increases off-axis, initial failure is expected to occur at a point along the ridge axis. Due to the higher Young's modulus toward the segment ends, stress builds up more rapidly in these regions under elastic extension of the plate. Competing with this stress accumulation pattern is the positive gradient in yield strength from segment center to the distal ends, which favors yielding to occur first at the segment center where the yield strength of the plate is minimum.

Based on this model we define two modes of fault development at ridge segments: faults that develop at the segment center and propagate outward, called Mode $\mathrm{C}$ (Center) faults (Figures 4-7a and 4-7c), and faults that develop at the segment ends and propagate inward, called Mode E (End) faults (Figures 4-7b and 4-7d). Mode C faults are expected to form at ridges where the along-axis variation in yield strength dominates the along-axis accumulation of stress. Conversely, Mode E faults are expected to develop in environments where the stress accumulation associated with the along-axis gradient in Young's modulus overcomes the variation in yield strength (Figures 4-7b and 4-7d). Therefore, in our linear model it is the interplay between $\mathrm{dE} / \mathrm{dy}$ and $\mathrm{d} \sigma_{\text {yield }} / \mathrm{dy}$ that determines whether failure is preferred at the segment center (Figures 4-7a and 4-7c) or at the segment ends (Figures 4$7 \mathrm{~b}$ and 4-7d). Figure 4-8 shows calculation results illustrating how the relationship between $\mathrm{dE} / \mathrm{dy}$ and $\mathrm{d} \sigma_{\text {yield }} / \mathrm{dy}$ controls the transition from Mode $\mathrm{C}$ to Mode $\mathrm{E}$ faults. The values for

Young's modulus and yield strength at the segment center $\left(\mathrm{E}^{\circ}=75 \mathrm{GPa}\right.$ and $\sigma_{\text {yield }}^{\circ}=50$ $\mathrm{MPa}$ ) are based on the depth-averaged Young's modulus and yield strength from the MAR OH-1 segment (Figures 4-4a and 4-4c). 

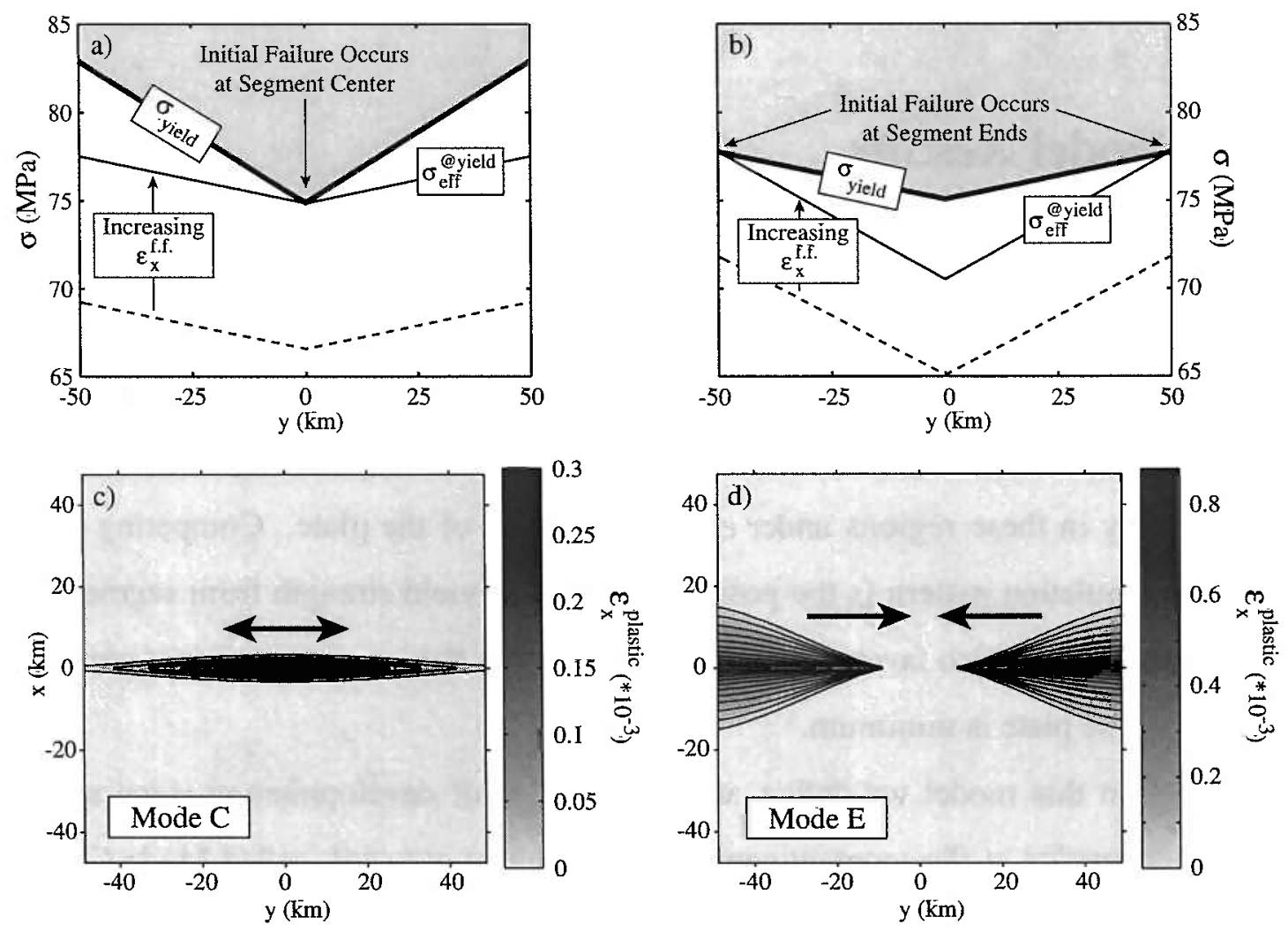

Figure 4-7: $(a, b)$ Illustration of the relationship between effective stress (thin line) versus yield stress (thick line) at the ridge axis. Failure will occur if the effective stress is greater than the yield stress at any point. Mode $C$ faults (a) develop when the effective stress exceeds the yield stress first at the ridge center. Dashed line illustrates the effective stress for a value of $\varepsilon_{\mathrm{x}}^{\mathrm{f} . f .}$ before yielding. Mode $\mathrm{E}$ faults (b) develop if the effective stress exceeds the yield strength first at the segment ends. The relative magnitude of the along-axis gradient in Young's modulus (which controls the gradient in effective stress for a given far-field strain) and yield strength dictate whether Mode $\mathrm{C}$ or Mode $\mathrm{E}$ faults will develop. (c) Calculated plastic strain associated with Mode $\mathrm{C}$ faults (propagation from the center of a segment toward its ends) calculated using the ADINA finite-element model. Arrows indicate direction of fault propagation. (d) Calculated plastic strain associated with Mode E faults (propagation from the segment ends toward the segment center). Note that the calculated plastic yield zone associated with Mode $\mathrm{E}$ faults is wider than for Mode $\mathrm{C}$ faults. 
The relation between $\mathrm{dE} / \mathrm{dy}$ and $\mathrm{d} \sigma_{\text {yield }} /$ dy also plays an important role in controlling the evolution of the plastic yield zone after failure (Figure 4-9). In the case where enhanced accumulation of stress toward the segment ends perfectly balances the along-axis gradient in yield strength, the plate will break uniformly along the entire ridge axis (Figure 4-9c). However as $\mathrm{dE} / \mathrm{dy}$ increasingly dominates $\mathrm{d} \sigma_{\text {yield }} / \mathrm{dy}$, the plastic zone associated with Mode $\mathrm{E}$ faults becomes more triangular in shape, and propagates less toward the segment center for a given value of $\varepsilon_{\mathrm{x}}^{\text {f.f. }}$ (Figures $4-9 \mathrm{a}$ and $4-9 \mathrm{~b}$ ). In contrast, as $\mathrm{d} \sigma_{\text {yield }} / \mathrm{dy}$ is increased relative to $\mathrm{dE} / \mathrm{dy}$, it becomes increasingly difficult for Mode $\mathrm{C}$ faults to propagate outward toward the segment ends (Figures 4-9d and 4-9e).

Another interesting prediction of this model is that the calculated plastic zone related to Mode $\mathrm{E}$ faults is significantly wider in across-axis dimension than the plastic zone associated with Mode $\mathrm{C}$ faults. This calculation suggests that Mode $\mathrm{E}$ faults would tend to initiate and remain active over a broader across-axis region than Mode $\mathrm{C}$ faults. Several segments of the slow-spreading MAR have been documented to exhibit hour-glass shaped rift valleys [e.g., Sempéré et al., 1993; Detrick et al., 1995; Weiland et al., 1996]. Although there is little geologic evidence constraining the width of the zone of fault initiation at ridge segments, Bohnenstiehl and Kleinrock [1999] used estimates of the amount of strain on individual faults to conclude that fault initiation is primarily confined within the median valley of a slow-spreading segment. If correct, this observation suggests that segments with hour-glass shaped rift valleys may be characterized by wider zones of fault initiation at the segment ends than at the center. Furthermore, Shaw [1992] and Shaw and Lin [1993] observed that faults near segment ends are typically characterized by greater amounts of throw than faults at segment centers. This observation could be explained if Mode $E$ faults remain active, continuing to accumulate slip for a longer time period than Mode $\mathrm{C}$ faults.

The results of our model are moderately sensitive to the base values chosen for Young's modulus, $\mathrm{E}^{\circ}$, and yield strength, $\sigma_{\text {yield }}^{\circ}$, at the segment center. The observed variations in these parameters with spreading rate and offset length (Figure 4-4) suggest the need to test the importance of $\mathrm{E}^{\circ}$ and $\sigma_{\text {yield }}^{\circ}$ in influencing the transition from Mode $\mathrm{C}$ to Mode $\mathrm{E}$ faults. Figure 4-10 shows the Mode C-E transition in $\mathrm{dE} / \mathrm{dy}$ versus $\mathrm{d} \sigma_{\text {yield }} /$ dy phase space for four 


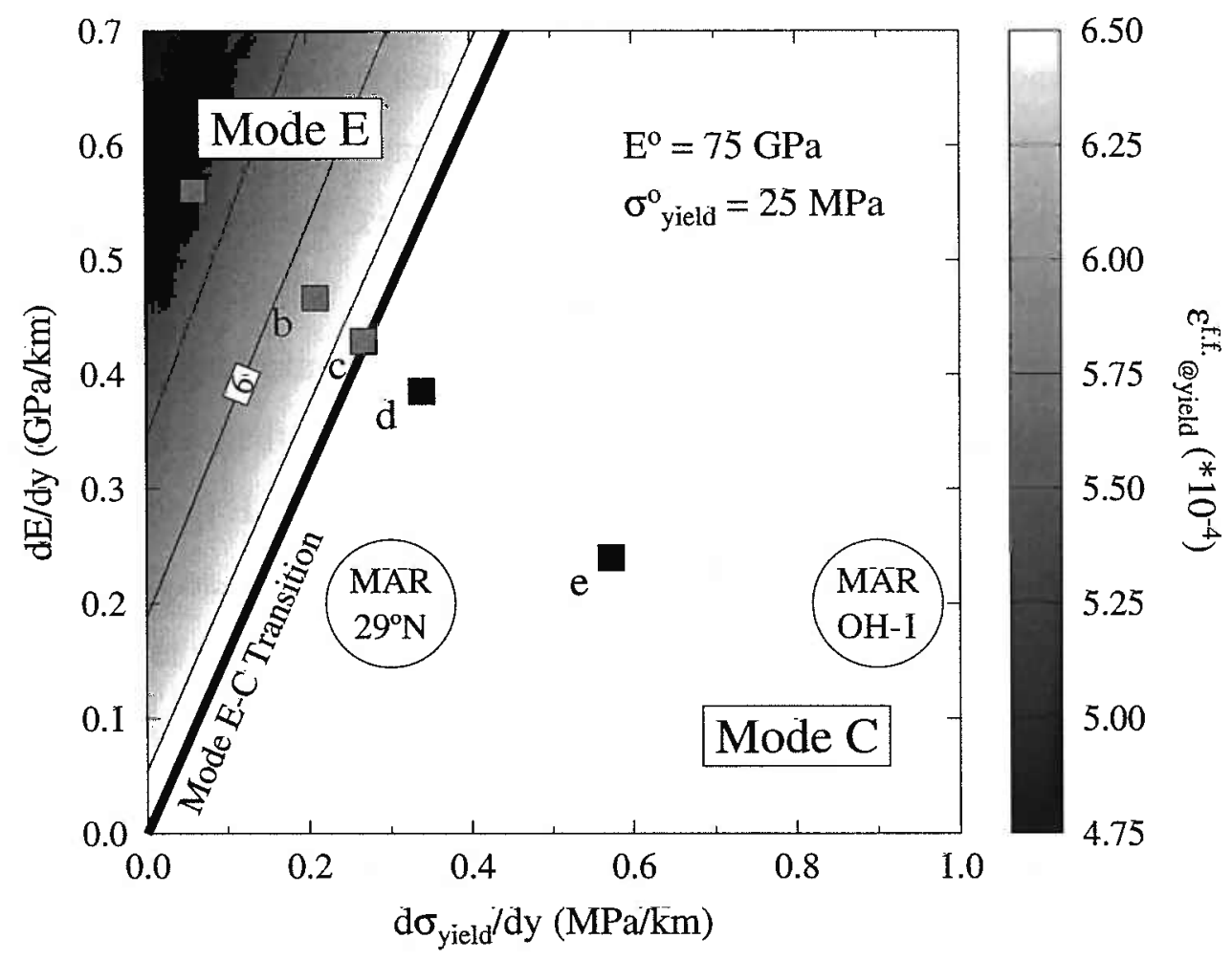

Figure 4-8: $\mathrm{dE} / \mathrm{dy}$ versus $\mathrm{d} \sigma_{\text {yield }} / \mathrm{dy}$ phase space showing the mode boundary between Mode $\mathrm{E}$ and Mode $\mathrm{C}$ faults (thick black line). Shaded contours illustrate the far-field strain necessary for initial yielding. The values of Young's modulus and yield strength at the center of the segment are $\mathrm{E}^{\circ}=75 \mathrm{GPa}$ and $\sigma_{\text {yield }}^{\circ}=50 \mathrm{MPa}$, respectively. Gray squares show parameters used in the 5 cases shown in Figure 4-9. Approximate parameter values of the $\mathrm{OH}-1$ and MAR $29^{\circ} \mathrm{N}$ segments are shown based on the values of $\mathrm{dE} / \mathrm{dy}$ and $\mathrm{d} \sigma_{\text {yield }} / \mathrm{dy}$ calculated in Figure 4-4. Note that when the along-axis gradient in yield strength dominates, Mode $\mathrm{C}$ faults are predicted. Conversely, when the along-axis gradient in Young's modulus dominates over the gradient in yield strength, Mode $\mathrm{E}$ faults are predicted. 

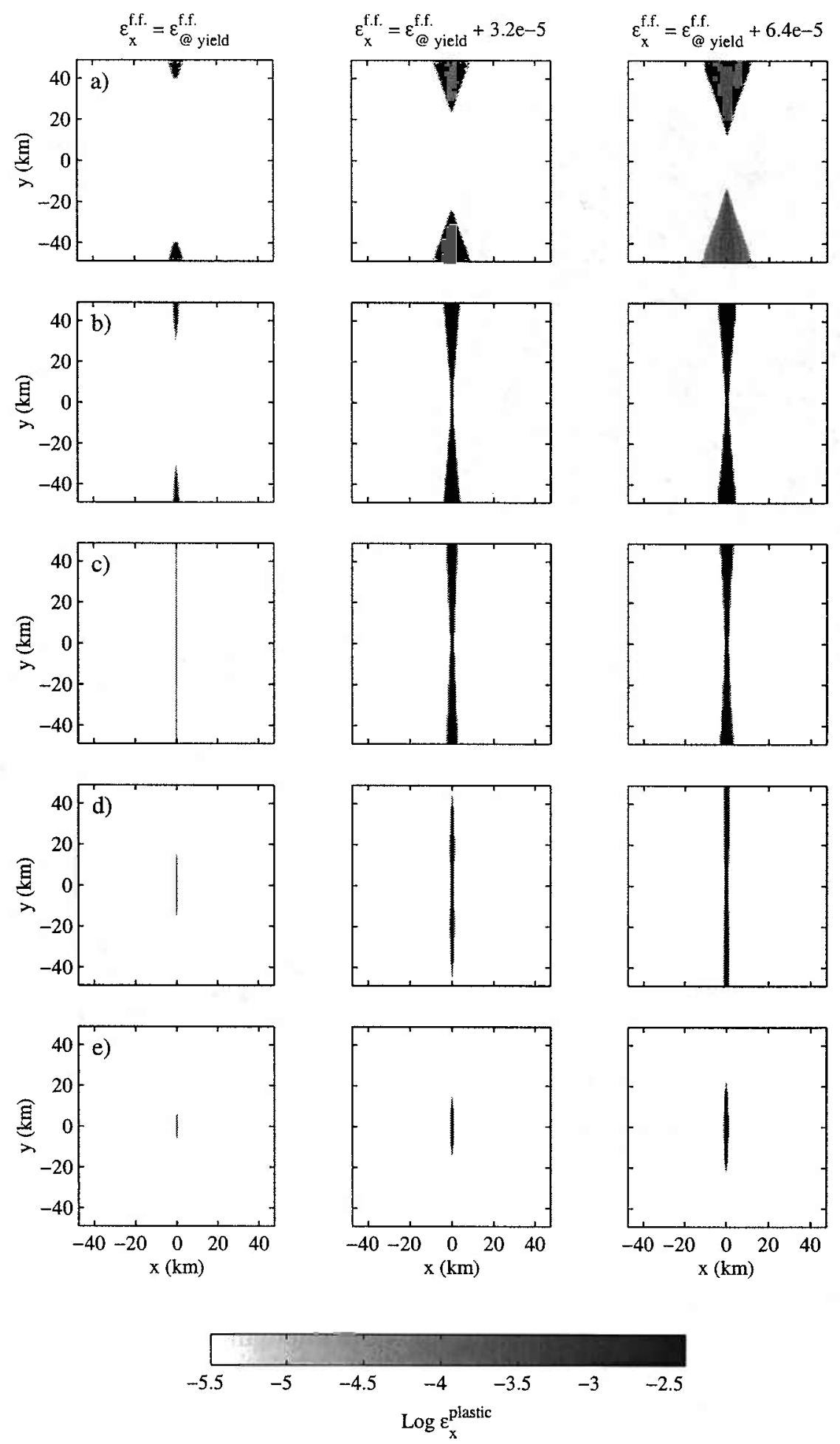

Figure 4-9: Plastic strain calculated using ADINA with increasing far-field strain for the 5 sets of parameters illustrated in Figure 4-8. (a,b) Mode E faults. (c) Uniform failure along the entire segment. $(d, e)$ Mode $C$ faults. For a given $\varepsilon_{x}^{\text {f.f. }}$, the plastic deformation zone propagates a greater distance along-axis for the parameters in 4-9b and 4-9d than in 4-9a and 4-9e. 

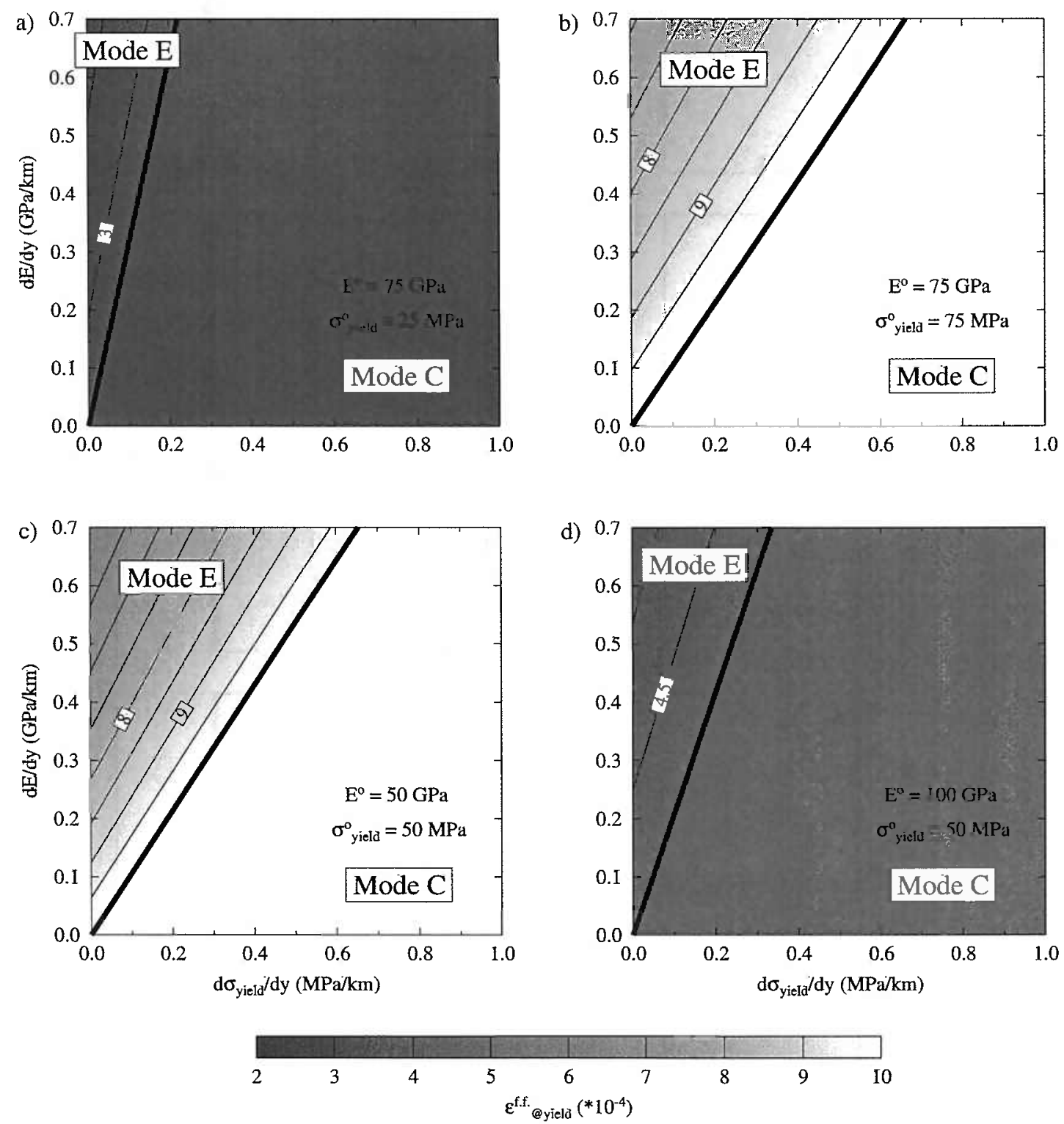

Figure 4-10: Mode boundary between Mode E and Mode C faults (thick black line) for four different values of Young's modulus and yield strength at the segment center. (a) $\mathrm{E}^{\circ}$ $=75 \mathrm{GPa}, \sigma_{\text {yield }}^{\circ}=25 \mathrm{MPa}$. (b) $\mathrm{E}^{\circ}=75 \mathrm{GPa}, \sigma_{\text {yield }}^{\circ}=75 \mathrm{MPa}$. (c) $\mathrm{E}^{\circ}=50 \mathrm{GPa}, \sigma_{\text {yield }}^{\circ}=$ $50 \mathrm{MPa}$. (d) $\mathrm{E}^{\circ}=100 \mathrm{GPa}, \sigma_{\text {yield }}^{\circ}=50 \mathrm{MPa}$. Shaded contours illustrate the far-field strain necessary for initial yielding. Note the decrease in size of the Mode $\mathrm{E}$ phase space as the value of $\mathrm{E}^{\circ}$ becomes larger relative to the value of $\sigma_{\text {yield }}^{\circ}$ 
different combinations of $\mathrm{E}^{\circ}$ and $\sigma_{\text {yield }}^{\circ}$. Note that as $\sigma_{\text {yield }}^{\circ}$ is decreased relative to $\mathrm{E}^{\circ}$, the slope of the Mode C-E transition increases and the region of phase space for Mode $E$ faults decreases. In addition, the amount of far-field extensional strain required for initial yielding increases with increasing $\sigma_{\text {yield }}^{\circ}$ and decreasing $\mathrm{E}^{\circ}$.

\subsection{Discussion}

\subsubsection{Observations of Mode E Faults}

At slow-spreading ridges new normal faults typically initiate within the rift valley where the lithosphere is thinnest [Searle, 1984; Bicknell et al., 1987; Carbotte and Macdonald, 1990]. Once formed, these faults grow in the along-axis direction through a combination of lateral propagation and linkage with other faults [e.g., Cowie, 1998], while simultaneously being rafted off-axis due to the injection of dikes at the ridge axis. The point of maximum throw on a fault scarp represents the location at which the fault is preferentially reactivated over time, by slip events of higher frequency or larger magnitude. Several authors have argued that the location of maximum throw can be used as a proxy for the point of fault initiation [Barnett et al., 1987; Walsh and Watterson, 1987]. If correct, this implies that many of the large faults observed toward the end of slow-spreading segments may not only accumulate slip preferentially in these locations, but may also initiate at or near the segment ends.

Figure 4-1a illustrates an excellent example of a large Mode E fault on inside-corner crust of the MAR OH-1 segment (oriented $\mathrm{N} 45^{\circ} \mathrm{E}$ from $35^{\circ} \mathrm{N}$ to $35^{\circ} 10^{\prime} \mathrm{N}$ ), with maximum throw near the segment end and tapering toward the segment center. Similarly, a series of discrete Mode $\mathrm{E}$ faults (oriented $\mathrm{N} 45^{\circ} \mathrm{E}$ just south of $34^{\circ} \mathrm{N}$ ) can be seen on the inside corner crust of segment OH-3 (Figure 4-1b). Another MAR segment that displays several prominent Mode E faults is at $25^{\circ} 10^{\prime} \mathrm{N}$ (Figure 4-2). Here faults are observed to accumulate maximum slip at different locations along the segment, not only at the center and ends, but also in between. However, toward the ends of the segment large faults appears to be 
preferred on inside-corner crust. Other examples of Mode E faults have been documented elsewhere along the MAR [Smith et al., 1995; Searle et al., 1998; Escartín et al., 1999; Briais et al., 2000].

\subsubsection{Factors Favoring the Generation of Mode $E$ Faults}

Figures 4-8 and 4-10 show the conditions under which along-axis changes in Young's modulus can generate large enough gradients in stress to overcome the increased lithospheric strength of the segment ends, thus forming Mode $E$ faults. We note that the observed values of $\mathrm{dE} / \mathrm{dy}$ along the MAR OH- 1 and MAR $29^{\circ} \mathrm{N}$ segments are insufficient to generate the stress gradients necessary to produce the observed Mode E faults in these locations (Figure 4-8). Figure 4-11 shows the additional gradient in effective stress that is required to switch from Mode $\mathrm{C}$ to Mode $\mathrm{E}$ faulting as a function of $\mathrm{dE} / \mathrm{dy}$ and $\mathrm{d} \sigma_{\text {yield }} / \mathrm{dy}$. Note that the closer a segment plots to the Mode C-E transition in Figure 4-11, the smaller the additional along-axis gradient in stress that is necessary to switch from one mode of faulting to the other.

However, changes in Young's modulus are not the only source of along-axis variations in stress at a ridge segment. Furthermore, segments are rarely characterized by just one mode of faulting, as would be predicted if variations in $\mathrm{dE} / \mathrm{dy}$ and $\mathrm{d} \sigma_{\text {yield }} /$ dy were the only parameters controlling fault development at a ridge segment. This observation suggests that temporal variations in stress must also play an important role in the dynamics of faulting at mid-ocean ridges. Below we discuss several mechanisms that may lead to the development of Mode $\mathrm{E}$ faults at a ridge segment.

\section{Serpentinization}

One hypothesis that has been proposed to explain the generation of Mode $\mathrm{E}$ faults is that serpentinites may be sufficiently abundant toward the end of slow-spreading segments to significantly reduce the strength of the lithosphere in these locations [Escartin et al., 1997]. If serpentinites are present in large enough quantities to offset the gradient in lithospheric strength caused by the change in thermal and crustal structure along a segment, it could 


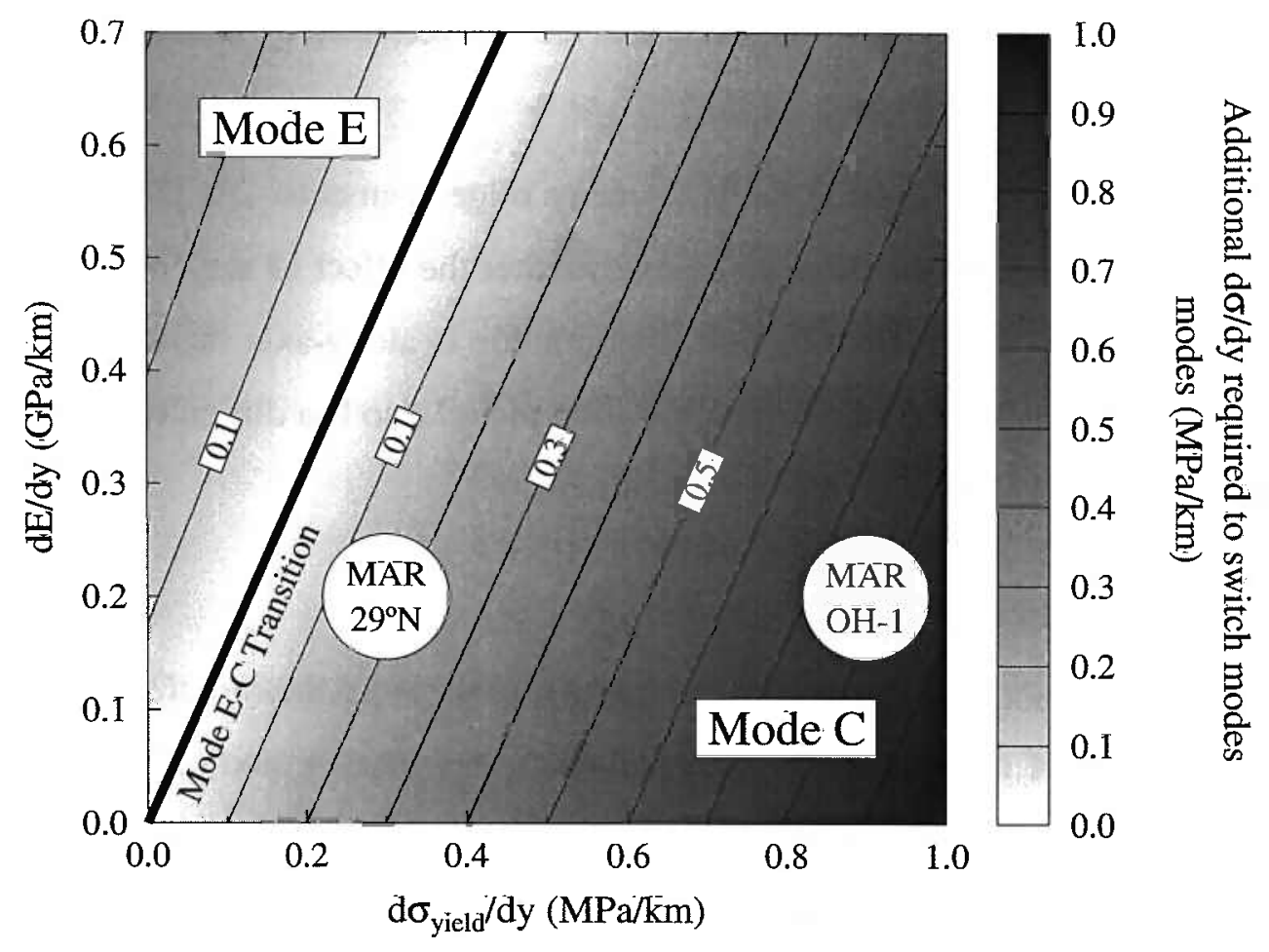

Figure 4-11: Contour plot of the additional gradient in $\sigma_{\text {eff }}$, after accounting for variations due to $\mathrm{dE} / \mathrm{dy}$, that is necessary to switch modes of faulting. Approximate parameter values of the OH-1 and MAR $29^{\circ} \mathrm{N}$ segments are shown based on the values of $\mathrm{dE} / \mathrm{dy}$ and $\mathrm{d} \sigma_{\text {yield }} / \mathrm{dy}$ calculated in Figure 4-4. We hypothesize that the additional gradient in $\sigma_{\text {eff }}$ necessary to form the Model E faults observed at the OH-1 and MAR $29^{\circ} \mathrm{N}$ segments may be generated by a combination of shearing along transform faults and temporal variations in magma supply along the ridge axis. 
Figure 4-12: (facing page) (a) Contour plot of the calculated change in effective stress, $\Delta \sigma_{\mathrm{RT}}$, generated by applying a shear stress, $\sigma_{\mathrm{T}}$, along two $30-\mathrm{km}$-long transform faults. We assume $\sigma_{\mathrm{R}}=\sigma_{\text {yield }}^{\circ}$ and set $\sigma_{\mathrm{T}}$ such that the stress coupling ratio, $\sigma_{\mathrm{R}} / \sigma_{\mathrm{T}}$, is equal to 2 . (b,c,d) Along-axis variations in effective stress, with $\varepsilon_{\mathrm{x}}^{\text {f.f. }}=6.4 \mathrm{e}-4$, for $\sigma_{\mathrm{R}} / \sigma_{\mathrm{T}}$ ratios of 2,3 , and 5 , respectively. Solid black lines show the variation in $\sigma_{\text {eff }}$ for the stress-free boundary conditions shown in Figure 4-6 (i.e., $\sigma_{\mathrm{T}}=0$ ). Gray lines illustrate $\sigma_{\text {eff }}$ at $\mathrm{x}=0,-5,-15$, and $-25 \mathrm{~km}$, respectively. Note that except for the small region surrounding the point at which the ridge and transform meet, the inside corners are characterized by increased $\sigma_{\text {eff }}$ relative to the outside corners.

explain the formation of Mode $\mathrm{E}$ faults at many ridge segments. At present, however, there are not sufficient observations to assess whether the effect of serpentinization alone is sufficient to offset the gradient in yield strength due to along-axis variations in thermal and crustal structure. Further, the serpentinization model also has difficulty explaining the asymmetry observed at inside- and outside-corners.

\section{Transform Fault Shearing}

An alternative mechanism that may increase stresses toward the end of a spreading segment is the effect of shear stress resisting relative plate motion along a transform fault. Phipps Morgan and Parmentier [1984] and Grindlay and Fox [1993] modeled the stress field associated with transform offsets by applying varying ratios of the tensile stress resisting plate separation at the ridge axis, $\sigma_{\mathrm{R}}$, and the shear stress resisting relative plate motion along the offset, $\sigma_{\mathrm{T}}$. The tensile stresses predicted by these models are amplified at the inside corners, with their magnitude increasing with greater offset lengths and smaller $\sigma_{\mathrm{R}} / \sigma_{\mathrm{T}}$ ratios. Pollard and Aydin [1984] predict a similar increase in stress at an inside corner without imposing any shear stress along the transform boundary, by modeling the propagation of two over-lapping cracks in an elastic plate.

Figure 4-12 illustrates the effect of imposing a shear stress resisting relative plate motion along a $30-\mathrm{km}$ section of the top and bottom boundaries of the model space adjacent to the ridge axis. Assuming $\sigma_{\mathrm{R}}=\sigma_{\text {yield }}^{\circ}$, we vary $\sigma_{\mathrm{T}}$ to show the change in effective stress associated with $\sigma_{\mathrm{R}} / \sigma_{\mathrm{T}}$ ratios of 2,3 , and 5 (Figures 4-12b, 4-12c, and 4-12d, respectively). Although for numerical simplicity we choose to vary only $\sigma_{\mathrm{T}}$, we note that in reality changes 

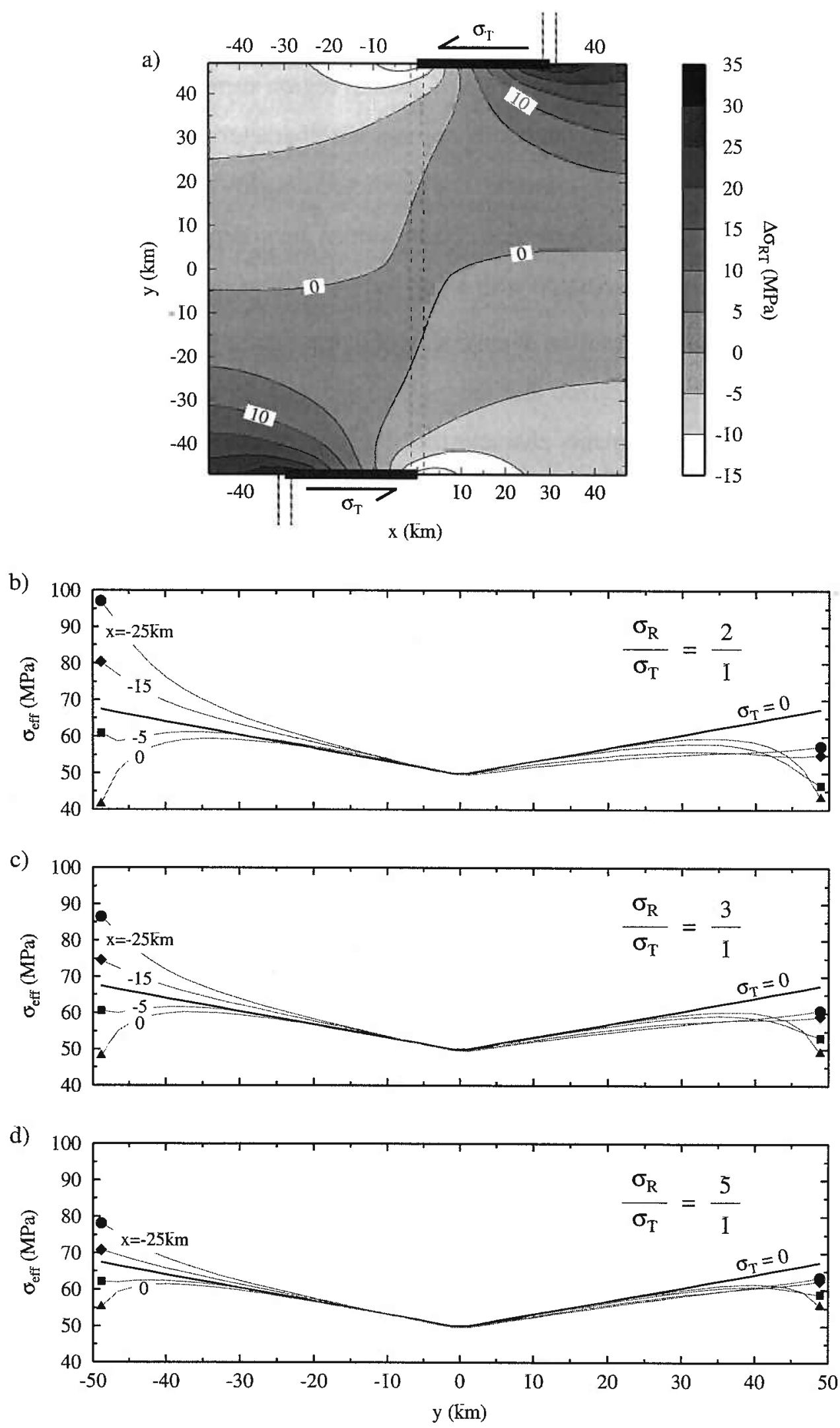
in $\sigma_{\mathrm{R}} / \sigma_{\mathrm{T}}$ can be caused by variations in $\sigma_{\mathrm{R}}$ during waxing and waning phases of ridge axis magma supply. With the minor exception of a small region surrounding the point at which the ridge and transform meet, the inside corners are characterized by increased effective stresses relative to the outside-corners. The predicted $\mathrm{d} \sigma_{\text {eff }} /$ dy are up to $\sim 0.3 \mathrm{MPa} / \mathrm{km}$ at a distance of $15 \mathrm{~km}$ from the ridge axis. These values are comparable in magnitude to the predicted stress changes associated with along-axis variations in Young's modulus.

Based on the observed rotation of normal fault scarps near non-transform offsets, Grindlay and Fox [1993] hypothesized that the $\sigma_{\mathrm{R}} / \sigma_{\mathrm{T}}$ ratio will generally fall in the range of 3 to 5 . However, along segments characterized by low residual gravity and shallow axial bathymetry, the observed morphology was found to be more closely matched by a $\sigma_{\mathrm{R}} / \sigma_{\mathrm{T}}$ ratio of 1 to 3. Grindlay and Fox [1993] proposed that the decrease in $\sigma_{\mathrm{R}} / \sigma_{\mathrm{T}}$ might be caused by a reduction in $\sigma_{\mathrm{R}}$ related to periods of robust magmatic activity. If correct, this could indicate a link between enhanced magmatism at the segment center and increased tectonism at the inside corners.

This prediction is consistent with observations from the MAR OH-1 and OH-3 segments. Residual gravity anomalies [Detrick et al., 1995] and seismic refraction profiles [Hooft et al., 2000; Canales et al., 2000b] show the OH-1 segment to be magmatically robust, with young, sheet-like lava flows covering the axial valley floor at the segment center [Gràcia et al., 1999]. Toward the segment ends the valley floor shows extensive faulting and fissuring, and a large Mode $\mathrm{E}$ fault is observed at the northeast inside corner (Figure 41a). In contrast, the $\mathrm{OH}-3$ segment is characterized by a weaker, less stable magma source, offset to the south of the segment center [Gràcia et al., 1999]. At OH-3 a series of small Mode $\mathrm{E}$ faults is observed at the inside corners, while the zone of most intense faulting and fissuring is near the segment center (Figure 4-1b). Note that while the calculated asymmetry in stresses between the inside and outside corners depends on the assumed $\sigma_{\mathrm{R}} / \sigma_{\mathrm{T}}$ ratio, the predicted stresses are always higher at the inside-corner crust than at the outside-corner and segment center. Thus transform shearing enhances the probability of forming Mode $\mathrm{E}$ faults on inside-corner crust. 


\section{Temporal Variations in Stresses}

Episodic periods of enhanced magmatic activity have been inferred from off-axis residual gravity anomalies on time scales of 2-5 m.y. [Pariso et al., 1995; Tucholke et al., 1997]. Moreover, a recent across-axis seismic profile at the MARK Area of the MAR suggested even shorter fluctuations in magma supply, with periods of 400-800 k.y. [Canales et al., 2000a]. Thermal and crustal structure are critical in the determination of $\mathrm{dE} / \mathrm{dy}$ and $\mathrm{d} \sigma_{\text {yield }} / \mathrm{dy}$, and have been shown to vary as a function of magma supply [e.g., Tucholke et al., 1997; Canales et al., 2000a]. Thus, temporal variations in magmatic accretion at slow-spreading ridge axes may influence not only the ridge-transform coupling ratio $\sigma_{\mathrm{R}} / \sigma_{\mathrm{T}}$ as discussed above, but also the elastic and mechanical properties of the lithosphere.

In addition to variations due to magma supply, the process of faulting itself affects the local stress field. Stress drops associated with large earthquakes range from 1 to $10 \mathrm{MPa}$ [Kanamori and Anderson, 1975]. A normal fault releases stress to either side of its center, but concentrate stress at its tips [e.g., Pollard and Aydin, 1984; Crider and Pollard, 1998]. As a population of normal faults evolves, the stress fields associated with individual faults interact, leading to the coalescence of multiple small faults into a few larger structures [e.g., Cowie et al., 1993; Tuckwell et al., 1998]. As this process continues, extension due to the linkage of existing faults will eventually begin to dominate over the nucleation of new faults [Spyropoulos et al., 1999; Ackermann and Schlische, 1999]. These complications are beyond the scope of this study, but illustrate that the stress field at a mid-ocean ridge segment changes constantly as new faults form and deformation continues on existing fault planes.

In summary, we suggest that along-axis variations in Young's modulus, shear stresses along a transform fault, and temporal variations in magma supply and fault growth can all lead to enhanced stresses toward the end of a segment. While any of these factors alone may be insufficient to generate a Mode E fault, together they can produce large enough stresses at the segment ends to overcome the positive along-axis gradient in yield strength. 


\subsubsection{Fault Initiation at Fast-Spreading Ridges}

At slow-spreading MAR segments, variations in thermal and crustal structure cause $\mathrm{dE} / \mathrm{dy}$ and $\mathrm{d} \sigma_{\text {yield }} / \mathrm{dy}$ to change significantly along-axis. In contrast, at the fast-spreading EPR Young's modulus and yield strength are observed to remain relatively constant along-axis (Figure 4-4). The lack of along-axis gradients in $\mathrm{dE} / \mathrm{dy}$ and $\mathrm{d} \sigma_{\text {yield }} / \mathrm{dy}$ at the EPR would tend to favor uniform failure along the ridge axis, rather than the development of prominent Mode $\mathrm{C}$ or $\mathrm{E}$ faults. This prediction is consistent with observations of faulting at the EPR, which show numerous small, closely-spaced faults forming continuously along the entire spreading segment [e.g., Carbotte and Macdonald, 1994; Alexander and Macdonald, 1996].

\subsection{Conclusions}

In this study we present a thin-plate model of a ridge segment to examine the relative importance of lateral changes in stress accumulation and the mechanical strength of the brittle lithosphere at a mid-ocean ridge spreading center. Thermal and seismic velocity models along the MAR OH- 1 and MAR $29^{\circ} \mathrm{N}$ segments show that both Young's modulus and yield strength are expected to change considerably along a slow-spreading segment, while little variation in these parameters is observed at the fast-spreading EPR $9^{\circ} \mathrm{N}$ segment. Higher values of depth-averaged Young's modulus toward the segment ends are calculated to lead to enhanced stress accumulation in these regions. Competing with this accumulation of stress is a positive gradient in yield strength from segment center to segment ends.

Based on this model we define two modes of fault development at slow-spreading segments: Mode C (Center) faults, which develop at the segment center and propagate outward, and Mode E (End) faults, which develop at the segment ends and propagate inward. Mode $\mathrm{C}$ faults are predicted to form in ridge environments where the along-axis variation in yield strength dominates the along-axis variation in stress accumulation. Conversely, Mode E faults are predicted to develop in environments where enhanced stress accumulation to- 
ward the segment ends overcomes the variation in yield strength. The plastic deformation zone associate with Mode $\mathrm{E}$ faults is predicted to be broader in across-axis extent than that of Mode $\mathrm{C}$ faults, potentially indicating that Mode $\mathrm{E}$ faults will initiate and remain active over a wider across-axis region.

High resolution mapping of the slow-spreading Mid-Atlantic Ridge has shown both Mode $\mathrm{C}$ and Mode $\mathrm{E}$ faults to be prevalent features of many segments, with large Mode E faults typically forming on inside-corner crust. Our calculations show that along-axis variations in Young's modulus alone do not appear to be sufficient to generate the stress gradients necessary for Mode E faulting at many slow-spreading segments. Therefore, we propose a model in which temporal changes in magma supply affect both along-axis gradients in the stresses and mechanical properties of the lithosphere and the stress conditions along the transform fault. During periods of enhanced magmatic activity, the ratio of ridge-to-transform stress, $\sigma_{\mathrm{R}} / \sigma_{\mathrm{T}}$, is predicted to decrease, generating larger stresses at inside corners and concentrating Mode $\mathrm{E}$ faulting in these locations. The lack of strong along-axis gradients in stress and lithospheric strength at fast-spreading ridges is predicted to generate a more uniform pattern of faulting as observed at the East Pacific Rise. The results of this study illustrate that the interplay between the along-axis variations in stress state and the mechanical properties of the lithosphere play an important role in controlling the style of fault development at a mid-ocean ridge spreading segment. 


\subsection{Thin-plate stress solution for linearly varying Young's modulus}

\subsubsection{Stress and Strain within the Plate}

In this section we present an analytical solution for stress and strain in a thin elastic plate with a linear gradient in Young's modulus from the center of a segment to its distal ends (see Figure 4-6). For a plate in plane stress, the stress, $\sigma$, and strain, $\varepsilon$, components can be written as

$$
\begin{array}{r}
\sigma_{\mathrm{x}}=\sigma_{\mathrm{x}}(\mathrm{x}, \mathrm{y}) \\
\sigma_{\mathrm{y}}=\sigma_{\mathrm{y}}(\mathrm{x}, \mathrm{y}) \\
\tau_{\mathrm{xy}}=\tau_{\mathrm{xy}}(\mathrm{x}, \mathrm{y}) \\
\tau_{\mathrm{xz}}=\tau_{\mathrm{yz}}=\sigma_{\mathrm{z}}=0
\end{array}
$$

We define a coordinate system with the $\mathrm{x}$-axis oriented across the ridge axis and the $\mathrm{y}$-axis aligned along the ridge axis. The dimensions of the model space are $2 x_{\circ}$ and $2 y_{\circ}$. Small displacements, $\Delta \mathrm{u}_{\mathrm{x}}$ and $-\Delta \mathrm{u}_{\mathrm{x}}$, are imposed at the right- and left-hand sides of the model space, respectively. The top and bottom boundaries are assumed to be free slip

$$
\tau_{\mathrm{xy}}=\left.0\right|_{\text {at } \mathrm{y}= \pm \mathrm{y}_{\mathrm{o}}}
$$

with no y-displacement

$$
\mathrm{u}_{\mathrm{y}}=\left.0\right|_{\text {at } \mathrm{y}= \pm \mathrm{y}_{\mathrm{o}}}
$$

The stress and strain relationships for a thin-plate in plane stress [Jaeger and Cook, 1979] can be written as 


$$
\varepsilon_{\mathrm{x}}=\frac{1}{\mathrm{E}(\mathrm{y})}\left[\sigma_{\mathrm{x}}-\nu \sigma_{\mathrm{y}}\right]
$$

and

$$
\varepsilon_{\mathrm{y}}=\frac{1}{\mathrm{E}(\mathrm{y})}\left[\sigma_{\mathrm{y}}-\nu \sigma_{\mathrm{x}}\right]
$$

where $\nu$ is Poisson's ratio and E(y) is Young's modulus, which varies linearly along the y-axis

$$
\mathrm{E}(\mathrm{y})=\mathrm{E}^{\circ}+(\mathrm{dE} / \mathrm{dy})|\mathrm{y}|
$$

where $\mathrm{dE} / \mathrm{dy}$ is a constant. Note that because there is no across-axis gradient in Young's modulus, the far-field strain, $\varepsilon_{\mathrm{x}}^{\mathrm{fff}}=\Delta \mathrm{u}_{\mathrm{x}} / \mathrm{x}_{\mathrm{o}}$, is constant at all points in the model space for a given time step. Since no $y$-displacement is allowed along the top and bottom boundaries, at any $x$ value the integrated strain in the $y$-direction must sum to zero

$$
\int_{-\mathrm{y}_{\mathrm{o}}}^{\mathrm{y}_{\mathrm{o}}} \varepsilon_{\mathrm{y}} \mathrm{dy}=0
$$

Solving Equations 4.5, 4.6, and 4.8 under the condition that $\sigma_{\mathrm{y}}$ remains constant, we arrive at the following expressions for stress and strain within the plate for any given farfield strain, $\varepsilon_{\mathrm{x}}^{\mathrm{f.f}}$.

$$
\begin{gathered}
\sigma_{\mathrm{y}}=\frac{\nu \varepsilon_{\mathrm{x}}^{\mathrm{f.f}}(\mathrm{dE} / \mathrm{dy}) \mathrm{y}_{\mathrm{o}}}{\left(1-\nu^{2}\right) \ln \left[\frac{(\mathrm{dE} / \mathrm{dy}) \mathrm{y}_{\mathrm{o}}-\mathrm{E}^{\circ}}{\mathrm{E}^{\circ}}\right]} \\
\sigma_{\mathrm{y}}(\mathrm{x})=\left[(\mathrm{dE} / \mathrm{dy})|\mathrm{y}|+\mathrm{E}^{\circ}\right] \varepsilon_{\mathrm{x}}^{\text {f.f. }}-\nu \sigma_{\mathrm{y}} \\
\varepsilon_{\mathrm{y}}(\mathrm{y})=\left[\frac{1-\nu^{2}}{(\mathrm{dE} / \mathrm{dy})|\mathrm{y}|+\mathrm{E}^{\circ}}\right] \sigma_{\mathrm{y}}-\nu \varepsilon_{\mathrm{x}}^{\text {f.f. }}
\end{gathered}
$$

We note that $\sigma_{\mathrm{x}}$ and $\varepsilon_{\mathrm{y}}$ are solely functions of $\mathrm{y}$. Due to the stress-free boundary conditions 
and uni-axial extension applied to the model, the shear-stresses are negligible. Numerical models show that the addition of an across-axis gradient in Young's modulus, $\mathrm{dE} / \mathrm{dx}$, with

$$
\frac{\int_{-x_{0}}^{x_{o}} E(x, 0) d x}{2 x_{0}}=E^{0}
$$

will generate effective stresses at the ridge-axis which differ by less than $0.25 \%$ from the case of $\mathrm{dE} / \mathrm{dx}=0$, for values of $\mathrm{dE} / \mathrm{dx}$ similar to that observed at segment $\mathrm{OH}-1$ (Figure 4-5a).

\subsubsection{Calculation of Initial Failure}

Perfect plasticity is characterized by a mechanical yield stress, $\sigma_{\text {yield }}$, beyond which permanent strain appears. In this study, we assume failure of a thin-plate will follow the Von-Mises yield criterion, defined as

$$
\sigma_{\text {eff }} \geq \sigma_{\text {yield }}
$$

where

$$
\sigma_{\mathrm{eff}}=\sqrt{\frac{1}{2}\left[\left(\sigma_{\mathrm{x}}-\sigma_{\mathrm{y}}\right)^{2}+\sigma_{\mathrm{x}}^{2}+\sigma_{\mathrm{y}}^{2}+6 \tau_{\mathrm{xy}}^{2}\right]}
$$

At any point in the plate the yield strength is assumed to be a linear function of $\mathrm{x}$ and $\mathrm{y}$, with

$$
\begin{aligned}
\sigma_{\text {yield }}(\mathrm{x}, \mathrm{y})=\sigma_{\text {yield }}^{\mathrm{o}} & +\left(\mathrm{d} \sigma_{\text {yield }} / \mathrm{dx}\right)|\mathrm{x}| \\
& +\left(\mathrm{d} \sigma_{\text {yield }} / \mathrm{dy}\right)|\mathrm{y}|
\end{aligned}
$$

Because the across-axis gradient in yield strength, $\mathrm{d} \sigma_{\text {yield }} / \mathrm{dx}$, is positive and the effective stress, $\sigma_{\text {eff }}$, varies only in the y-direction (see Equations $4.9 \& 4.10$ ), initial yielding must 
occur at the ridge axis where $x=0$. Therefore, we can solve for the value of the far-field strain when yielding occurs, $\varepsilon_{@}^{\text {f.f. yield }}$, which is a function of y only:

$$
\varepsilon_{@ \text { yield }}^{\text {f.f. }}(y)=\frac{\sigma_{\text {yield }}(y)}{\sqrt{\mathrm{A}+\mathrm{B}+\mathrm{C}}}
$$

where

$$
\begin{array}{r}
\mathrm{A}=\left[(\mathrm{dE} / \mathrm{dy})|\mathrm{y}|+\mathrm{E}^{\circ}\right]^{2} \\
\mathrm{~B}=\frac{\left[(\mathrm{dE} / \mathrm{dy})|\mathrm{y}|+\mathrm{E}^{\circ}\right]\left(2 \nu^{2}-\nu\right)(\mathrm{dE} / \mathrm{dy}) \mathrm{y}_{\circ}}{\left(1-\nu^{2}\right) \ln \left[\frac{(\mathrm{dE} / \mathrm{dy})|\mathrm{y}|+\mathrm{E}^{\circ}}{\mathrm{E}^{\circ}}\right]} \\
\mathrm{C}=\frac{\left(\nu^{4}-\nu^{3}+\nu^{2}\right)(\mathrm{dE} / \mathrm{dy})^{2} \mathrm{y}_{\circ}^{2}}{\left(1-\nu^{2}\right)^{2} \ln ^{2}\left[\frac{(\mathrm{dE} / \mathrm{dy})|\mathrm{y}|+\mathrm{E}^{\circ}}{\mathrm{E}^{\circ}}\right]}
\end{array}
$$

This relationship is used to determine whether initial failure occurs at the segment center or segment ends, and thus whether Mode C or Mode E faults are preferred. 


\title{
Chapter 5
}

\section{3-D stress interaction between oceanic transform faults and mid-ocean ridges}

\begin{abstract}
Transform faults play a fundamental role in controlling tectonic processes at mid-ocean ridges. Faulting near the end of spreading segments is observed to be highly asymmetric, with normal faults formed at the inside corner (IC) characterized by greater throw and more oblique orientations than faults formed at the outside corner (OC). In this study we present the results of a series of 3-D boundary element calculations to investigate the effects of transform faults on stress state, fault development, and morphology at oceanic spreading centers. We find that on time scales longer than a typical earthquake cycle (i.e., $\sim 100$ years), transform faults behave as zones of significant weakness. Specifically, mechanical coupling of only $\sim 5 \%$ best explains the observed patterns of strike-slip and oblique normal faulting near a ridge-transform intersection. On time scales shorter than a typical earthquake cycle, we find that transient "locked" periods can produce anomalous reverse faulting similar to those mechanisms observed at the ICs of several slow-spreading ridge segments. Furthermore, we predict that extensional stresses will be suppressed at the IC due to the shear along the transform resisting ridge-normal extension. This implies that the greater fault throw and enhanced microseismicity observed at ICs cannot be explained by elevated extensional stresses in these regions. Instead, we propose a model in which fault growth at segment ends is related to the interaction between normal faults and the neighboring transform. Boundary element calculations show that normal faults that develop at an IC, adjacent to a weak transform fault, will experience preferential growth relative to faults forming at the OC away from the transform. This model provides a simple geometric explanation for enhanced fault growth and fault-related topography at the IC of a ridge-transform intersection.
\end{abstract}




\subsection{Introduction}

Transform and non-transform offsets (NTOs) are fundamental features of slow-spreading mid-ocean ridges. These offsets divide the ridge axis into individual spreading segments with lengths ranging from 10 to $100 \mathrm{~km}$ [e.g., Macdonald, 1986; Sempéré et al., 1993]. The steady-state topography of a slow-spreading ridge is characterized by a prominent axial valley 10-40 km wide and 1-3 km deep [Macdonald and Atwater, 1978; Karson et al., 1987; Grindlay et al., 1992; Sempéré et al., 1993] and is consistent with a lithospheric necking model [e.g., Tapponnier and Francheteau, 1978; Phipps Morgan et al., 1987; Phipps Morgan and Chen, 1993; Shaw and Lin, 1996]. Axial depth typically shallows toward the center of a ridge segment, with transform and NTOs intersecting the axis in topographic depressions at the segment ends. Near a ridge-transform intersection, axial topography becomes highly asymmetrical, with crust on the inside-corner (IC) elevated relative to that on the outside-corner (OC) [e.g., Karson and Dick, 1983; Severinghaus and Macdonald, 1988; Blackman and Forsyth, 1991; Mutter and Karson, 1992; Tucholke and Lin, 1994] (Figures 5-1 and 5-2). Similar asymmetry is observed at segments bounded by NTOs, although the magnitude of the topographic variations are typically less extreme [e.g., Tucholke et al., 1997]. Another characteristic feature of many ridge-transform intersections is the formation of a deep "nodal basin" near the segment end [Fox and Gallo, 1984]. These basins are located where the axial valley joins the transform valley and are typically bounded on all sides by normal faults [e.g., Karson and Dick, 1983; Macdonald et al., 1986] (Figure 5-2).

The style of tectonic faulting is observed to vary systematically along slow-spreading ridge segments. Near segment centers, where negative residual gravity anomalies [e.g., Kuo and Forsyth, 1988; Lin et al., 1990; Detrick et al., 1995] and seismic velocity structure [e.g., Tolstoy et al., 1993; Hooft et al., 2000] indicate crustal thickness to be greatest, normal faults are observed to be small, linear, and closely spaced. In contrast, toward segment ends faults become larger, more widely spaced and are often oriented obliquely to the ridge axis [Shaw and Lin, 1993; Searle et al., 1998; Escartín et al., 1999]. Specifically, faults propagating from the segment center into IC crust curve sharply in the offset 


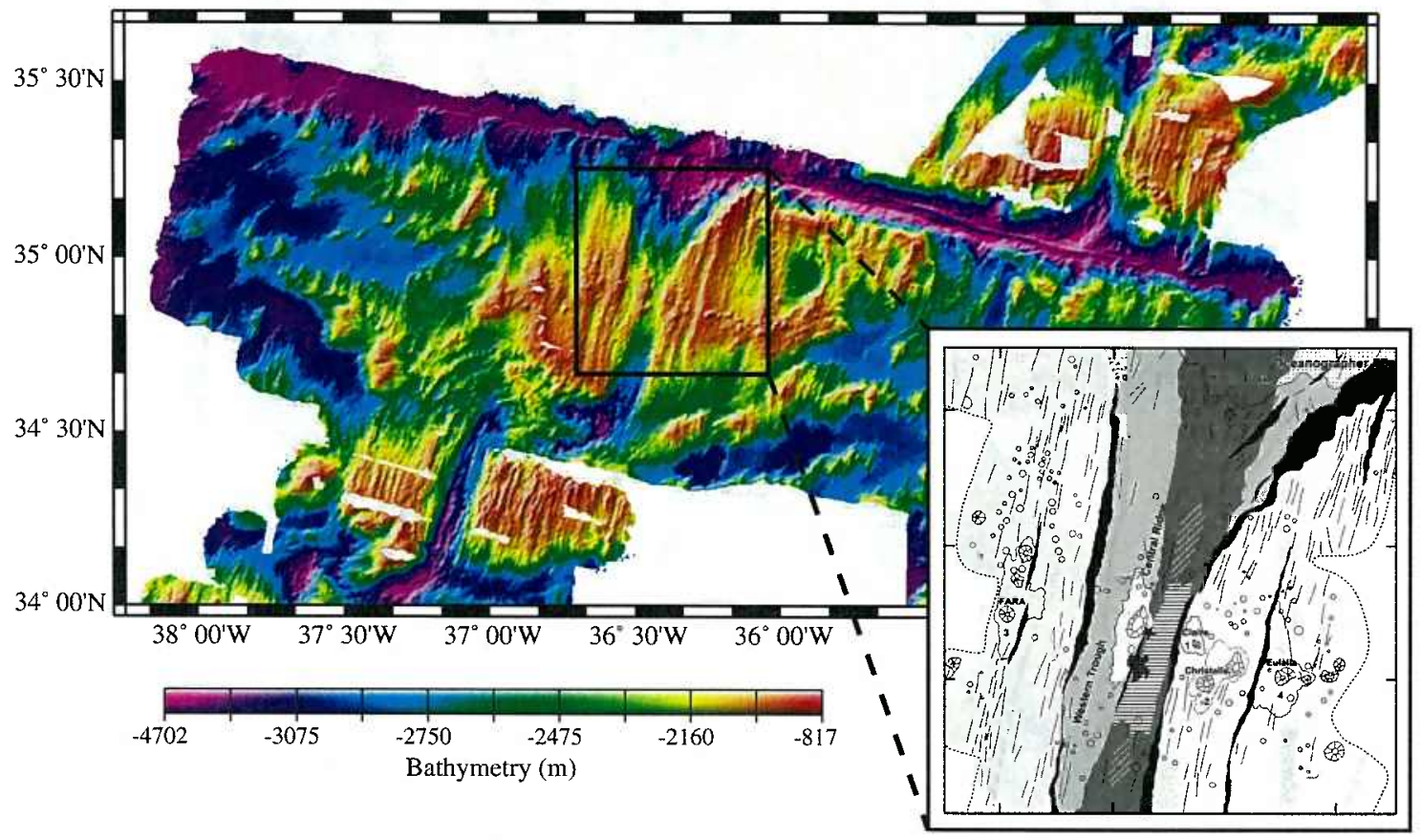

Figure 5-1: SIMRAD bathymetry map of the OH-1 and OH-2 segments south of the Oceanographer Fracture Zone at the Mid-Atlantic Ridge [Rabain et al., 2001]. Inset shows the geologic interpretation of the OH-1 segment by Gràcia et al. [1999] based on bathymetry, acoustic backscattering, submersible observations, and rock samples. Major fault scarps are shown in black. Figure adapted from Rabain et al. [2001] and Gràcia et al. [1999]. 


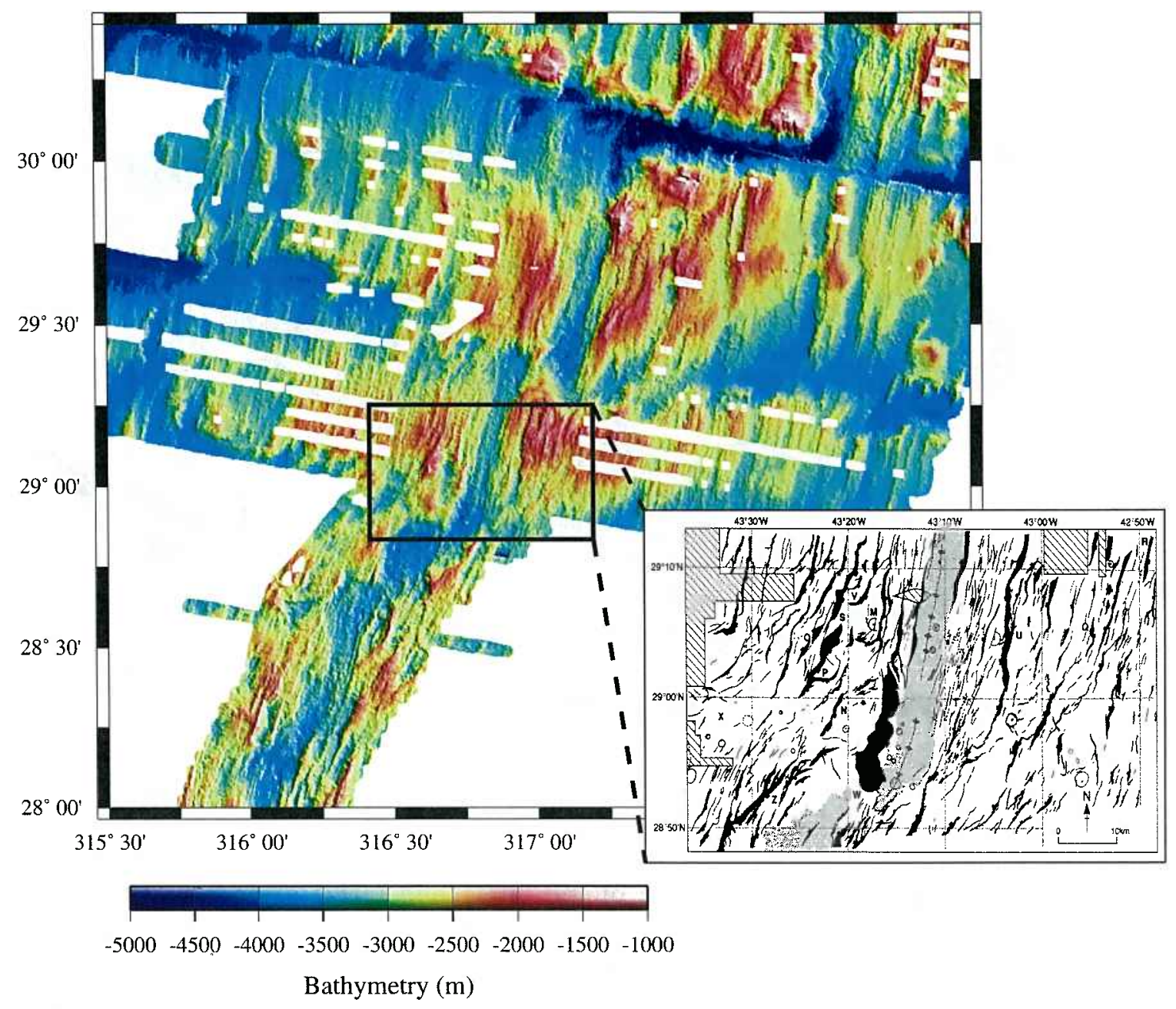

Figure 5-2: Sea Beam bathymetry map of the Mid-Atlantic Ridge south of the Atlantis Fracture Zone. Data from Purdy et al. [1990] and Sempéré et al. [1995]. Inset shows the geologic interpretation of the Broken Spur segment by Searle et al. [1998] based on bathymetry and TOBI sidescan images. Major fault scarps are shown in black. Inset from Searle et al. [1998]. 
direction, while faults propagating into OC crust typically remain parallel to the ridge [Karson and Dick, 1983; Macdonald et al., 1986; Searle et al., 1998]. These observations are consistent with teleseismic events indicating oblique- to transform-parallel normal faulting mechanisms near the end of several segments on the Mid-Atlantic Ridge (MAR) [Huang and Solomon, 1988; Wilcock et al., 1990]. Normal faults formed on the elevated IC crust are often characterized by greater throw than those formed on the OC [Severinghaus and Macdonald, 1988; Tucholke and Lin, 1994; Allerton et al., 1995; Escartín et al., 1999] and evidence from microearthquake studies [Rowlett and Forsyth, 1984; Wolfe et al., 1995; Barclay et al., 2001] and hydroacoustic monitoring [Smith et al., 2001] suggests that ICs may be more seismically active than OCs. ICs have also been associated with anomalous reverse faulting mechanisms, which are inconsistent with a ridge-normal extensional stress field [Engeln et al., 1986; Wolfe et al., 1993]. Wiens and Stein [1984] suggested that reverse faulting events observed off-axis in lithosphere older than $8 \mathrm{Myr}$ may be caused by ridge push forces. However, this mechanism is unlikely to be the source of the thrust events observed at ICs, as ridge push forces are predicted to be small near the ridge axis.

These observations suggest that ridge segmentation plays a fundamental role in controlling the tectonic processes at oceanic spreading centers. However, the mechanisms by which transform faults influence local topography and faulting are poorly understood. In this study we perform a series of three-dimensional boundary element calculations to examine the effects of transform faults on stress state, fault development, and morphology at oceanic spreading centers. Our investigation is divided into two sections. In the first section, we quantify the influence of transform coupling, offset length, and other parameters on the local stress state in oceanic lithosphere. Optimal fault planes are calculated as a function of the conditions on the transform and our results are discussed in relation to observed seismicity, transform strength, and fault orientation at ridge-transform intersections. In the second section, we examine the effect of a weak transform fault on the development of normal faults near segment ends. We predict asymmetry in slip distribution between faults located on IC and OC crust and discuss the implications for the formation of axial morphology. 


\subsection{Stress State Near Oceanic Transform Faults}

The asymmetry in seismicity and fault orientation near the ends of slow-spreading ridge segments is indicative of a deviation in the regional stress field from simple ridge-normal tension. Two mechanisms have been proposed to explain the rotation of normal faults near a ridge-transform intersection: 1) transform-normal extension, and 2) shearing along the active transform fault. Far-field extension across a transform has been proposed to explain the development of normal faults with transform-parallel orientations in transform valleys walls. The extreme rotation of these faults is not consistent with transform shearing, and requires an additional component of transform-normal tensile stress [Gudmundsson, 1995]. Pockalny et al. [1996] proposed that these tensile stresses are generated by changes in plate motion, and found that the uplifted transverse ridge along the Kane Fracture Zone was consistent with this model. However, while this mechanism may be applicable to specific geologic environments, it cannot explain the ubiquity of rotated normal faults at inside corners, as transform-normal stresses are unlikely to be universally present. Furthermore, transform-normal extension does not account for the asymmetry in fault orientation across a ridge-transform intersection.

An alternative mechanism to explain the rotation of normal faults at ICs is that shearing along the active transform fault locally perturbs the stress field at segment ends. This mechanism is supported by observations of rotated normal faults in pull-apart basins in continental terranes [e.g., Burchfiel and Stewart, 1966] and analogue experiments of deformation in clay-cake models [e.g., Courtillot et al., 1974; Macdonald et al., 1986]. These observations have been quantified by numerical studies that calculate the relative amount of stress rotation as a function of the ratio between the normal stress at the ridge axis and the shear stress along the transform [Fujita and Sleep, 1978; Phipps Morgan and Parmen-

tier, 1984; Grindlay and Fox, 1993]. These numerical studies characterized the lithosphere as a thin plate under plane stress. This approach requires that one of the three principal stresses remain vertical and equal to zero throughout the model space. However, in the regions surrounding the transform this assumption may not be valid. Furthermore, by spec- 
ifying stresses directly on the ridge axis and transform fault these models do not provide an estimate of the amount of stike-slip displacement that is coupled across the transform.

Below we present the results of a three-dimensional (3-D) boundary element analysis to quantify the spatial pattern of fault style surrounding a transform fault. Our numerical predictions are compared to observed fault patterns at slow-spreading ridges and the strength of oceanic transforms is assessed.

\subsubsection{Model Setup}

We model the stress field near a transform fault using the 3-D boundary element model of Gomberg and Ellis [1994]. This model uses the Green's functions derived by Okada [1992] to calculate internal deformation from shear and tensile dislocations in an elastic halfspace. The geometry of the model domain is defined by two ridge segments of length $L_{R}$ connected by a single transform fault of length $L_{T}$ (Figure 5-3a). Deformation is calculated within a volume of dimensions, $X_{o}$ (east-west), $Y_{o}$ (north-south), and $Z_{o}$ (vertical) (Table 5.1). The north, south, and basal boundaries of the model space are defined to be shear stress free with no normal displacement, while the top boundary is stress free.

Plate motion is simulated by specifying a displacement, $U_{o}$, on the east and west farfield boundaries of the model space and on both sides of each ridge axis (Figure 5-3b). Assuming a stress-free transform, this generates a total plate separation $2 \mathrm{U}_{\mathrm{o}}$, but no internal deformation in the plates for a totally decoupled transform fault. Seismic moment studies [Solomon et al., 1988] and measurements of cumulative fault throw from the Broken Spur segment of the Mid-Atlantic Ridge [Escartín et al., 1999] suggest that 15-20\% of sea-floor spreading is accommodated by extensional faulting with the rest by magmatic emplacement. Therefore, we superimpose on this displacement field a far-field extensional strain, $\varepsilon_{\text {f.f. }}$, of $0.20\left(2 \mathrm{U}_{\mathrm{o}} / \mathrm{X}_{\mathrm{o}}\right)$. The sensitivity of our results to the magnitude of $\varepsilon_{\text {f.f. }}$ will be discussed later in the text.

A relative displacement, $D_{s}$, is specified along the transform fault, with $D_{s}=2 U_{o}(1-\chi)$. The factor $\chi$ is used to simulate the coupling of the two plates across the transform, with 
a)

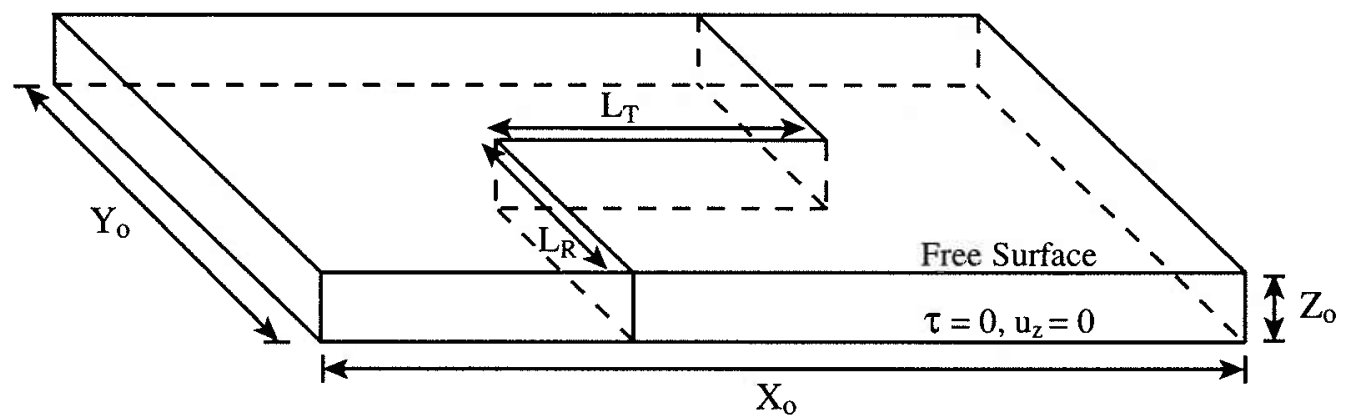

b)

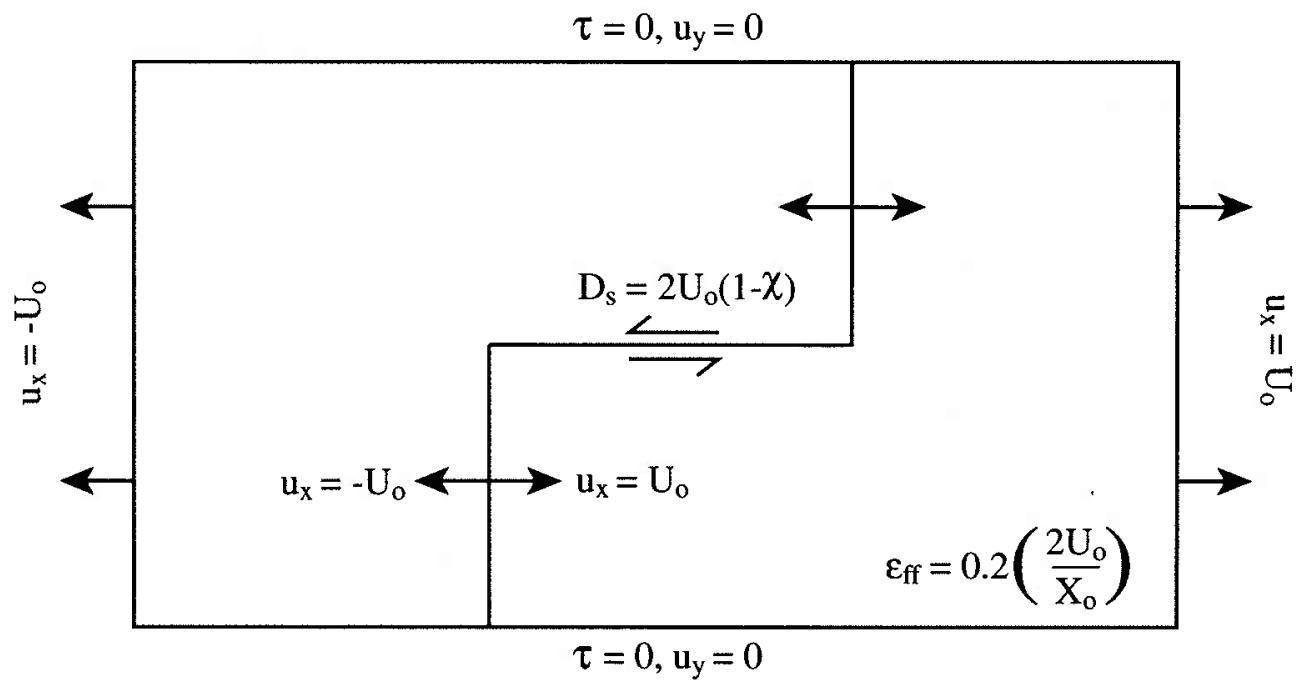

Figure 5-3: (a) Model setup and (b) boundary conditions for 3-D boundary element analysis. Deformation is driven by applying a displacement, $U_{o}$, to the east and west boundaries of the model space and on both sides of each ridge axis. Tectonic strain is simulated by superimposing a far-field extensional strain, $\varepsilon_{\text {f.f. }}=0.20\left(2 \mathrm{U}_{\mathrm{o}} / \mathrm{X}_{\mathrm{o}}\right)$. The effect of the transform coupling parameter, $\chi$, is simulated by specifying a relative displacement, $D_{s}$, along the transform, with $\mathrm{D}_{\mathrm{s}}=2 \mathrm{U}_{\mathrm{o}}(1-\chi)$. In this formulation, complete decoupling along the transform corresponds to $\chi=0$, while a completely locked fault is characterized by $\chi=1$. All calculations were performed with a Young's modulus, E, of $70 \mathrm{GPa}$ and a Poisson's ratio, $\nu$, of 0.25 . 
Table 5.1: Parameters used for modeling the stress field near a transform fault.

\begin{tabular}{llll} 
Symbol & Meaning & Value & Units \\
\hline$\nu$ & Poisson's ratio & 0.25 & \\
$\mathrm{E}$ & Young's modulus & 70 & $\mathrm{GPa}$ \\
$\mathrm{X}_{\mathrm{o}}$ & east-west model dimension & 240 & $\mathrm{~km}$ \\
$\mathrm{Y}_{\mathrm{o}}$ & north-south model dimension & 160 & $\mathrm{~km}$ \\
$\mathrm{Z}_{\mathrm{o}}$ & vertical dimension & $5-25$ & $\mathrm{~km}$ \\
$\mathrm{~L}_{\mathrm{R}}$ & ridge segment length & 80 & $\mathrm{~km}$ \\
$\mathrm{~L}_{\mathrm{T}}$ & transform length & $50-150$ & $\mathrm{~km}$ \\
$\mathrm{U}_{\mathrm{o}}$ & far-field displacement & 100 & $\mathrm{~m}$ \\
$\varepsilon_{\mathrm{f.f}}$ & far-field strain & & \\
$\chi$ & transform coupling parameter & $0-1$ & \\
$\sigma_{\mathrm{I}}, \sigma_{\mathrm{II}}, \sigma_{\mathrm{III}}$ & principal deviatoric stresses (tensile, neutrall, & & \\
& $\quad$ compressive) & & $\mathrm{MPa}$ \\
$\mu$ & coefficient of friction & 0 & \\
$\rho_{\mathrm{c}}, \rho_{\mathrm{m}}$ & crust, mantle density & 2900,3300 & \\
$v$ & spreading half rate & 0.01 & $\mathrm{~kg} \mathrm{~m}^{-3}$ \\
$\kappa$ & thermal diffusivity & $10^{-6}$ & $\mathrm{~m} \mathrm{yr}^{-1}$ \\
$\alpha$ & coefficient of thermal expansion & $3 \times 10^{-5}$ & ${ }^{\circ} \mathrm{C}^{-1}$ \\
$\mathrm{y}_{\text {lith }}$ & lithospheric plate thickness & & $\mathrm{km}^{-1}$ \\
\hline
\end{tabular}


Figure 5-4: (facing page) Principal deviatoric stress directions (a) $\sigma_{\mathrm{I}}$ (tension), (b) $\sigma_{\mathrm{II}}$ (neutral), and (c) $\sigma_{\mathrm{III}}$ (compression) calculated for a coupling parameter $\chi=0.15$ and a far-field displacement $U_{o}=100 \mathrm{~m}$. The model dimensions are $X_{o}=240 \mathrm{~km}, Y_{o}=160$ $\mathrm{km}$, and $\mathrm{Z}_{\mathrm{o}}=15 \mathrm{~km}$. Black lines indicate direction and relative magnitude of principal stresses resolved on a horizontal plane at a depth $4 \mathrm{~km}$. Thick grey lines show location of ridge segments $\left(\mathrm{L}_{\mathrm{R}}=80 \mathrm{~km}\right)$ and transform fault $\left(\mathrm{L}_{\mathrm{T}}=100 \mathrm{~km}\right)$. (d) Orientations of optimal fault planes calculated from the principal stresses. Color shading indicates the relative magnitude of $\tau_{\mathrm{dev}} / \sigma_{\mathrm{dev}+\text { lith }}$.

$\chi=0$ corresponding to total decoupling or free slip along the transform, and $\chi=1$ representing a completely locked fault. In reality, coupling across a transform fault will vary as a function of time. Immediately before a large earthquake the transform will behave in a more locked manner, exerting larger shear stresses on the adjacent plates. In contrast, after a major earthquake the accumulated shear stresses are released and the transform will behave in a more decoupled manner. By comparing the fault patterns predicted for various coupling parameters to observations from oceanic spreading centers, we can estimate the time-averaged strength of transform faults.

\subsubsection{Numerical Results}

Figure 5-4 illustrates the deviatoric stress field calculated for a $100-\mathrm{km}$ transform fault with $\chi=0.15$ under a far-field displacement, $U_{0}$, of $100 \mathrm{~m}$. This far-field displacement is equivalent to 10,000 years of spreading at a half-rate of $1 \mathrm{~cm} / \mathrm{yr}$. The principal deviatoric stresses are evaluated at a depth of $4 \mathrm{~km}$, which is consistent with the average depth of faulting near the axis of the MAR from microseismicity studies [Toomey et al., 1985; Kong et al., 1992; Wolfe et al., 1995; Barclay et al., 2001]. However, we note that the direction and magnitude of the principal stresses do not vary significantly with depth.

The predicted principal deviatoric stress orientations are used to estimate optimal fault planes throughout the model space (Figure 5-4d). According to Anderson's theory of faulting [Anderson, 1951], sliding is predicted on conjugate planes whose normals form an angle $\theta$ with the direction of maximum tensile stress. The angle $\theta$ is related to the coefficient of friction $\mu$ by 


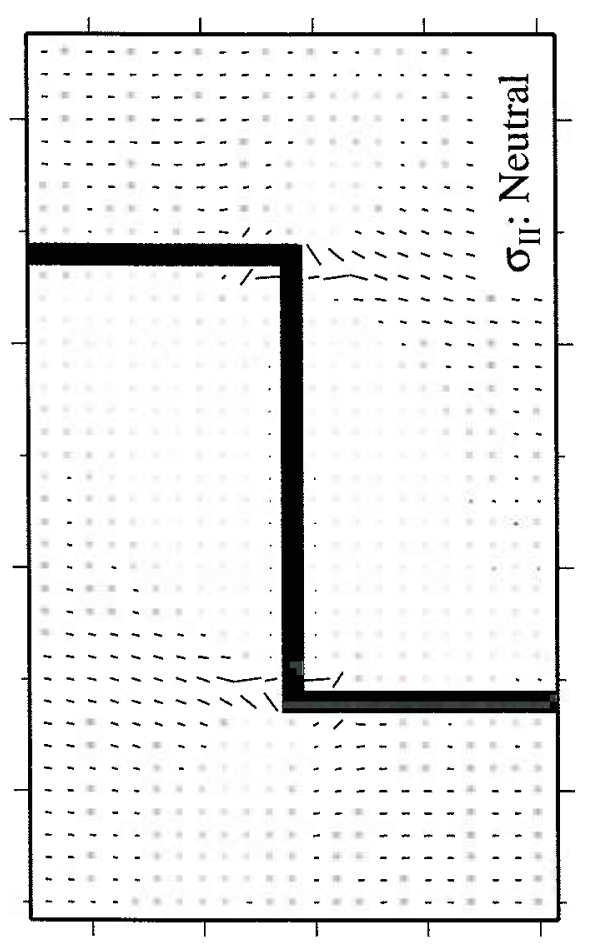

0

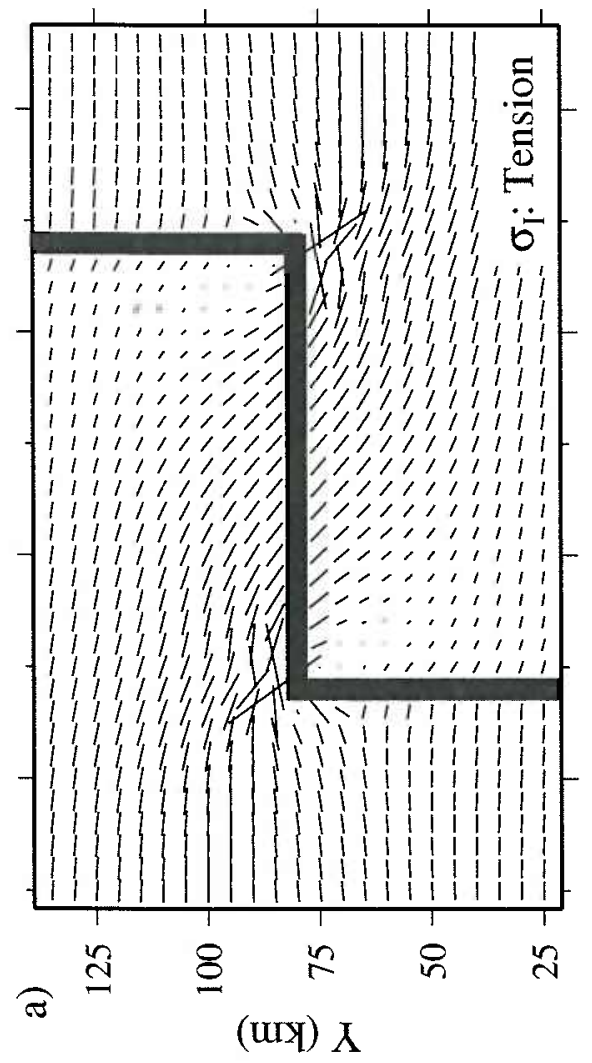

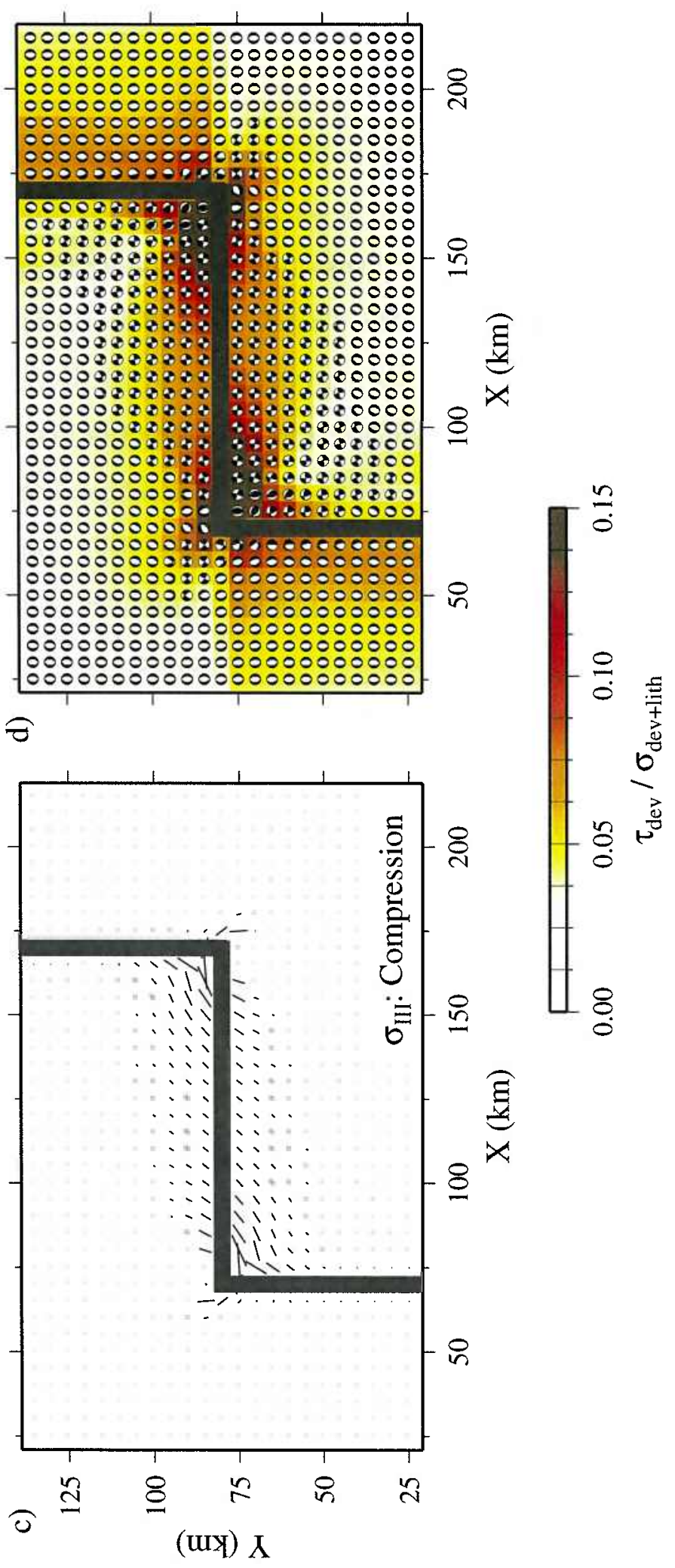




$$
\theta=\frac{1}{2} \tan ^{-1}\left(\frac{1}{\mu}\right)
$$

Seismic observations of focal mechanisms at slow-spreading ridges show that most normal faulting events occur at dip angles of $45^{\circ}$ and most strike-slip events are oriented parallel to the trend of the transform fault [Huang et al., 1986; Jemsek et al., 1986; Huang and Solomon, 1987, 1988; Thatcher and Hill, 1995]. Following Anderson's theory, these observations imply that the coefficient of friction in oceanic lithosphere is very low, and we use $\mu=0$ to calculate the orientation of failure planes throughout the model space. Note that had a higher coefficient of friction been used it would result in steeper dip angles for the predicted normal faults (Equation 5.1). Because we are considering a linear elastic solid, the directions and relative magnitudes of the principal deviatoric stresses will remain constant for all values of $U_{0}$. Therefore, the orientation of the optimal fault planes is independent of the magnitude of the far-field displacement. The reason for this is that both $\varepsilon_{\text {f.f }}$ and $D_{s}$ scale linearly with the far-field displacement. Thus, varying $U_{o}$ does not change the relative components of tensile to shear stress throughout the model space.

In order to assess the relative potential for failure on the predicted fault planes, we calculate the ratio of shear to normal stress resolved on the predicted planes using

$$
\frac{\tau_{\mathrm{dev}}}{\sigma_{\mathrm{dev}+\mathrm{lith}}}=\frac{\frac{\left(\sigma_{\mathrm{I}}-\sigma_{\mathrm{III}}\right)}{2}}{\frac{\left(\sigma_{\mathrm{I}}+\sigma_{\mathrm{III}}\right)}{2}+\sigma_{\mathrm{lith}}},
$$

where $\sigma_{\mathrm{I}}$ and $\sigma_{\mathrm{III}}$ are the maximum tensile and compressive deviatoric stresses and $\sigma_{\text {lith }}$ is the lithostatic pressure. Because the average depth of seismicity is observed to scale with the thickness of the oceanic lithosphere [Chen and Molnar, 1983], we approximate $\sigma_{\text {lith }}$ throughout the model space as a function of depth to the $600^{\circ} \mathrm{C}$ isotherm,

$$
\sigma_{\text {lith }}=\int_{0}^{y_{\text {lith }}} \rho \mathrm{g} \mathrm{dz}
$$

where $\rho$ is density and $\mathrm{g}$ is the acceleration due to gravity. $\mathrm{y}_{\text {lith }}$ represents the thickness of the lithosphere at $600^{\circ} \mathrm{C}$, 


$$
\mathrm{y}_{\text {lith }}=2 \sqrt{\frac{\kappa \mathrm{x}}{v}} \operatorname{erf}^{-1}\left(\frac{600^{\circ} \mathrm{C}}{1300^{\circ} \mathrm{C}}\right)
$$

where $\kappa$ is the thermal diffusivity and $v$ is the spreading rate. Because the lithosphere is observed to have a finite thickness at the ridge axis, the minimum value of $y_{\text {lith }}$ is set to be $6 \mathrm{~km}$. Note that while the orientations of the optimal fault planes were independent of the far-field displacement, $\tau_{\mathrm{dev}} / \sigma_{\mathrm{dev}+\text { lith }}$ increases with increasing values of $\mathrm{U}_{\mathrm{o}}$ due to the larger magnitude of the deviatoric stresses.

\section{Transform Coupling}

We now examine the effect of transform coupling on the predicted deviatoric stress field. In the case of complete decoupling $(\chi=0)$, no shear stresses are generated along the transform and the only source of deviatoric stress is ridge-normal tension generated from the far-field strain (Figure 5-5a). The calculated optimal fault planes are uniformly normal faulting mechanisms with ridge parallel orientations, and the magnitude of $\tau_{\mathrm{dev}} / \sigma_{\mathrm{dev}+\mathrm{lith}}$ is greatest near the ridge axes, where the lithosphere is thinnest (Figure 5-5a).

Increasing $\chi$ to 0.05 generates shear stresses along the transform, resulting in a number of changes in the predicted pattern of stress (Figure 5-5b). First, the orientation of the predicted normal faulting mechanisms at the IC and along the transform are rotated clockwise with respect to the spreading ridge. Second, a zone of strike-slip faulting forms near the ends of the transform, extending $\sim 20 \mathrm{~km}$ along the transform and $\sim 10 \mathrm{~km}$ along the IC side of the ridge axis. Finally, the magnitude of $\tau_{\operatorname{dev}} / \sigma_{\mathrm{dev}+\mathrm{lith}}$ on the predicted normal fault planes becomes asymmetrical across the ends of the ridge segments, with higher values predicted on the OCs. This somewhat surprising effect is caused by the induced shear along the transform resisting the far-field extensional stresses. Immediately adjacent to the ridge-transform intersection these shear stresses are sufficient to generate a reverse faulting mechanism, although the value of $\tau_{\mathrm{dev}} / \sigma_{\mathrm{dev}+\text { lith }}$ on the predicted fault plane is quite low.

As the coupling parameter, $\chi$, is increased to values of 0.10 and 0.15 , strike-slip deformation is predicted to dominate near the transform (Figures 5-5c and 5-5d). For $\chi=0.15$, 
Figure 5-5: (facing page) Effect of the coupling parameter, $\chi$, on the style of faulting near an oceanic transform fault. Orientations of calculated optimal fault planes for (a) $\chi=0$, (b) $\chi=0.05$, (c) $\chi=0.10$, and (d) $\chi=0.15$ after a far-field displacement $U_{0}=100 \mathrm{~m}$. The model dimensions are $X_{0}=240 \mathrm{~km}, Y_{0}=160 \mathrm{~km}$, and $Z_{o}=15 \mathrm{~km}$. Thick grey lines show location of ridge segments and transform fault. Color shading indicates the relative magnitude of $\tau_{\mathrm{dev}} / \sigma_{\mathrm{dev}+\text { lith }}$. A coupling parameter of 0.05 appears to most closely match the observed fault orientations and focal mechanisms at slow-spreading mid-ocean ridges.

strike-slip faulting is predicted in a $\sim 70-\mathrm{km}$ wide zone around the center of the transform ( $\mathrm{x}=120 \mathrm{~km}$ ) and to extend $\sim 50 \mathrm{~km}$ down the IC side of the ridge axis. Reverse faulting is predicted over a larger portion of the IC, with several of the fault planes associated with high values of $\tau_{\mathrm{dev}} / \sigma_{\mathrm{dev}+\text { lith }}$. The dominant style of deformation on the OC remains ridgeparallel normal faulting, with a slight counter-clockwise rotation in fault orientation near the segment end.

These results suggest that over time periods greater than a typical earthquake cycle ( $>100 \mathrm{yr}$ ), oceanic transform faults are characterized by small coupling parameters. Seismicity at oceanic spreading centers indicates that strike-slip faulting is confined to a narrow zone (20-25 km wide) around an active transform and is rarely observed along the axis of an adjacent spreading center [Engeln et al., 1986; Bergman and Solomon, 1988; Boettcher and Jordan, 2001]. Furthermore, the presence of large rotated normal faults at many ICs (e.g., Figure 5-1) indicates that significant extensional strain must be accommodated on these faults. These observations are most consistent with the numerical predictions for coupling parameters less than 0.10 .

On time scales shorter than a typical earthquake cycle (10-100 yr), coupling across oceanic transforms may experience significant temporal variability. Before a major earthquake we hypothesize that a transform will exhibit a larger coupling parameter. If correct, such "locked" periods could explain the presence of the anomalous reverse faulting mechanisms observed at a number of ICs [Engeln et al., 1986; Wolfe et al., 1993]. On such time scales, stresses may also vary as a function of individual magmatic events. The predicted pattern of stress is a function of the relative ratio of $\chi$ to the magnitude of the far-field 


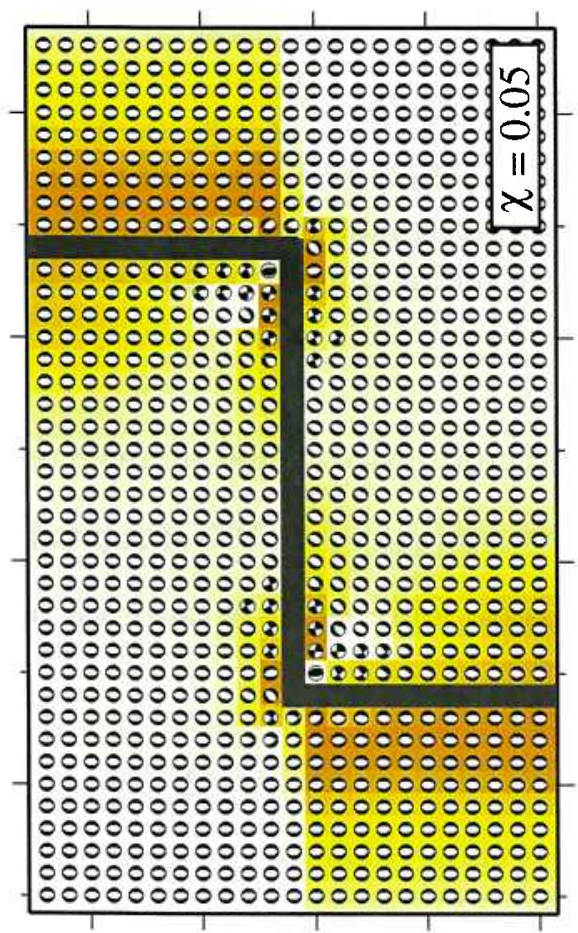

อ

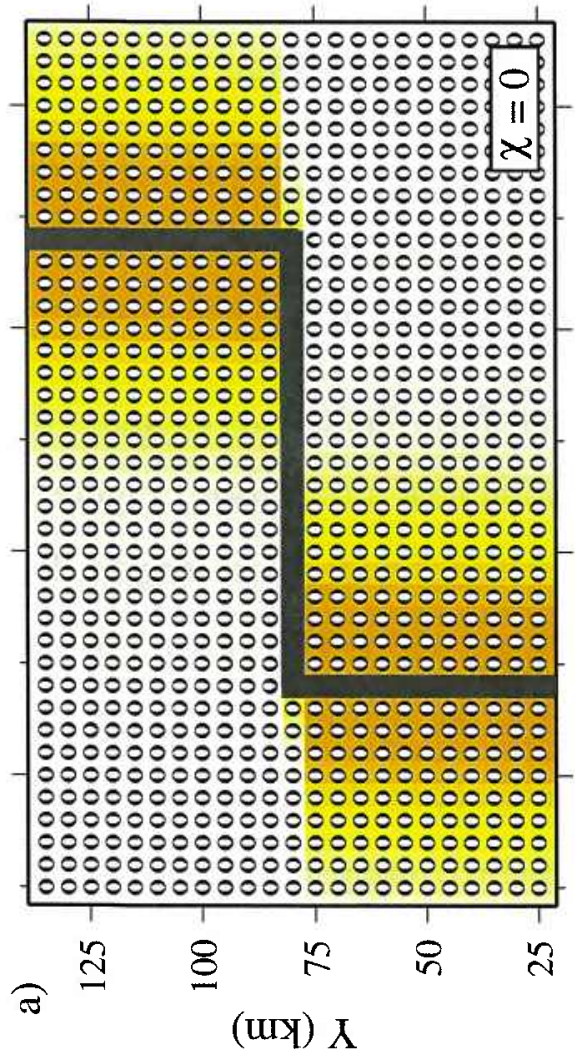

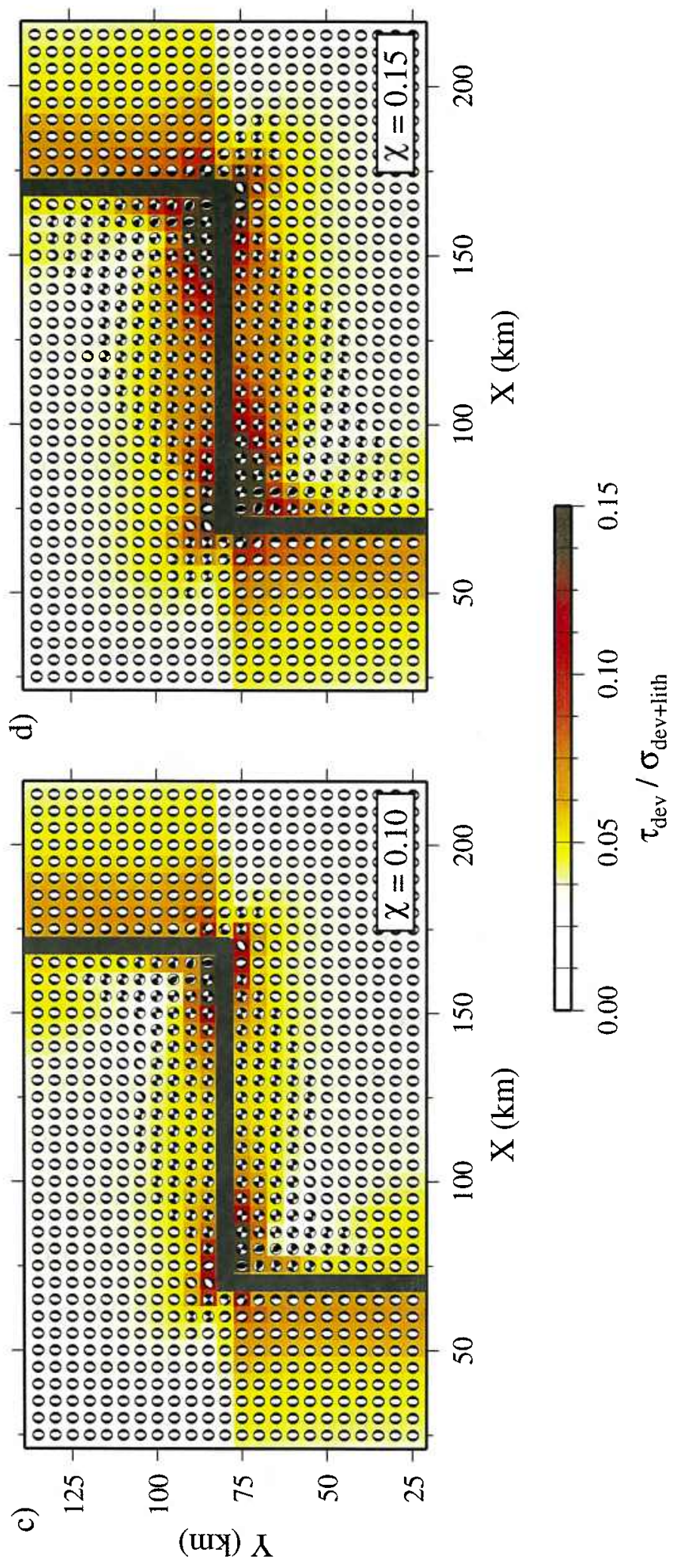

139 
Figure 5-6: (facing page) Effect of transform length on the style of faulting near an oceanic transform fault. Orientations of optimal fault planes for offset lengths of (a) $150 \mathrm{~km}$, (b) $100 \mathrm{~km}$, and (c) $50 \mathrm{~km}$, with $\chi=0.05$ and a far-field displacement $\mathrm{U}_{\mathrm{o}}=100 \mathrm{~m}$. Model dimensions are $X_{o}=240 \mathrm{~km}, Y_{o}=160 \mathrm{~km}$, and $Z_{o}=15 \mathrm{~km}$. Thick grey lines show location of ridge segments and transform fault. Color shading indicates the relative magnitude of $\tau_{\mathrm{dev}} / \sigma_{\mathrm{dev}+\mathrm{lith}}$. Note that the $50 \mathrm{~km}$ offset shows enhanced interaction of the stress fields along the transform fault.

extensional strain, $\varepsilon_{\text {f.f. }}$. For example, increasing $\varepsilon_{\text {f.f. }}$ by a factor of two would produce the same effect on fault style as decreasing $\chi$ by one-half. However, even for $50 \%$ amagmatic strain $\left(\varepsilon_{\text {f.f. }}=0.5\left(2 \mathrm{U}_{\mathrm{o}} / \mathrm{X}_{\mathrm{o}}\right)\right)$, the maximum coupling consistent with the observations is on the order of $\chi=0.10$.

We have compared our results with the numerical predictions of Phipps Morgan and Parmentier [1984], who found that the ratio of normal stress at the ridge axis to shear stress along the transform $\left(\sigma_{\mathrm{R}} / \sigma_{\mathrm{T}}\right)$ is in the range of 3 to 5 . For $\chi=0.05$, the ratio of $\sigma_{\mathrm{T}}$ at the center of the transform to $\sigma_{\mathrm{R}}$ at the ridge axis is $\sim 3.2$, while for $\chi=0.15$ this ratio is $\sim 1.1$. Thus, our estimates of transform coupling are consistent with those of Phipps Morgan and Parmentier [1984], showing that $\sigma_{\mathrm{R}} / \sigma_{\mathrm{T}}$ ratios of 3-5 correspond to mechanical coupling across a transform of $\leq 5 \%$ (i.e., $\chi \leq 0.05$ in our calculations).

\section{Transform Length}

Assuming $\chi=0.05$ is most appropriate for oceanic transform faults, we examine the effect of offset length on the stress field and style of faulting near a transform. Figure 56 illustrates the predicted pattern of faulting for three transforms with offset lengths, $L_{T}$, of 50, 100, and $150 \mathrm{~km}$, respectively. For offsets of 150 and $100 \mathrm{~km}$ (Figures 5-6a and 5-6b) there is little difference in the predicted orientation of the optimal fault planes and the magnitude of $\tau_{\mathrm{dev}} / \sigma_{\mathrm{dev}+\mathrm{lith}}$ near the ridge-transform intersection. In contrast, the $50 \mathrm{~km}$ offset is characterized by a larger region of predicted strike-slip faulting and elevated values of $\tau_{\mathrm{dev}} / \sigma_{\mathrm{dev}+\text { lith }}$ relative to the longer offsets (Figure 5-6c). A comparison of the predicted regions of strike-slip and reverse faulting for $\mathrm{L}_{\mathrm{T}}=50,100$, and $150 \mathrm{~km}$ is presented in Figure 5-7a. These results suggest that for offsets $\leq 50 \mathrm{~km}$ there is significant interaction 

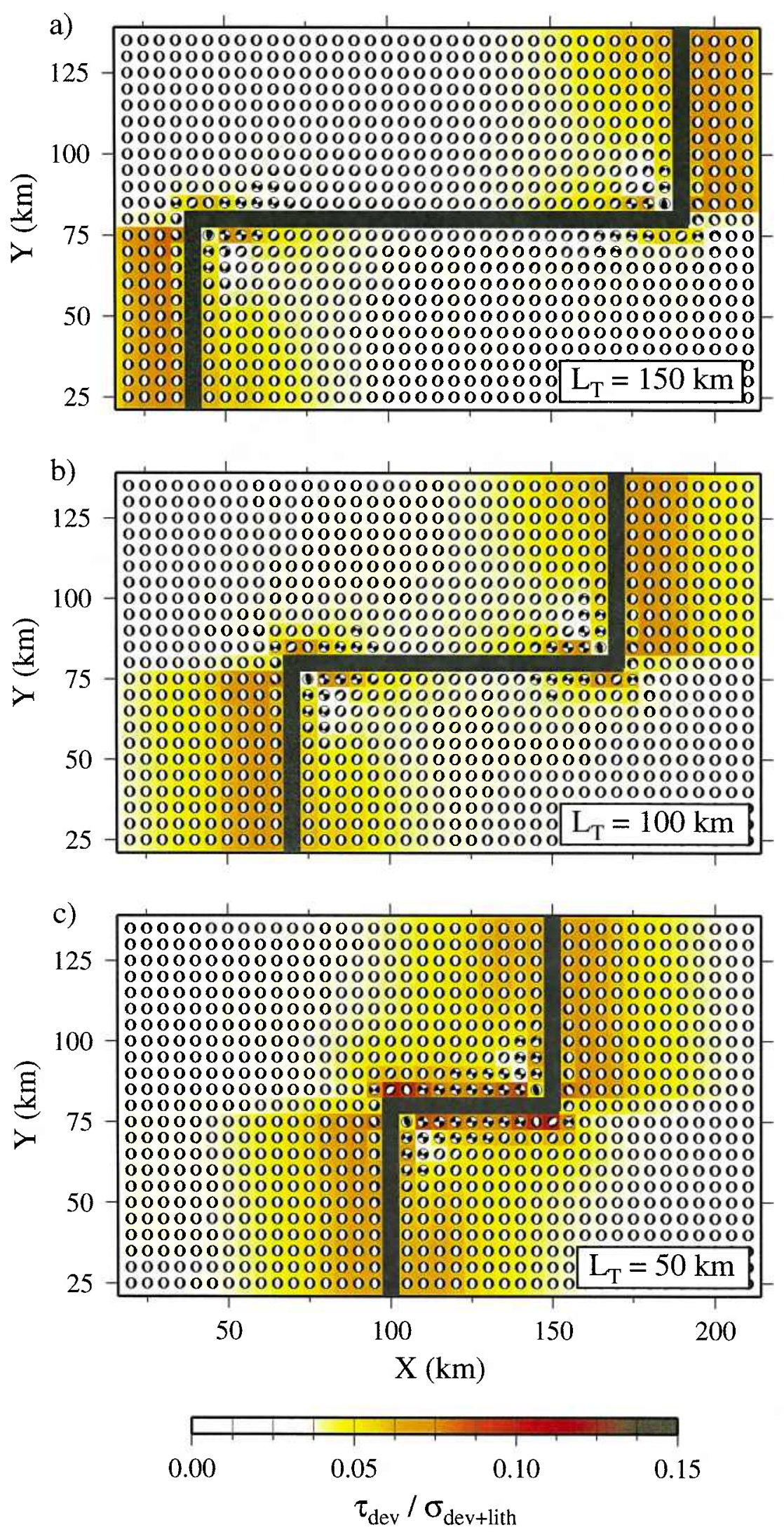

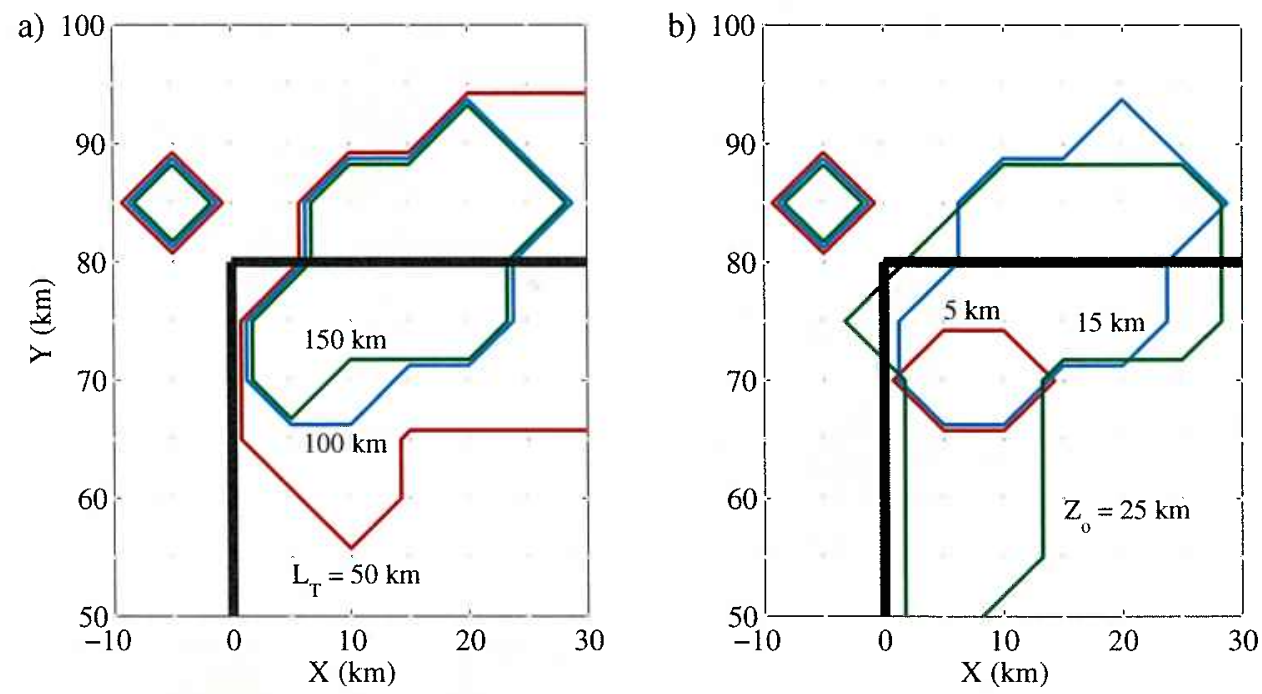

Figure 5-7: (a) Comparison of regions of predicted strike-slip and reverse faulting as a function of transform offset length. Red, blue, and green contours enclose areas of predicted strike-slip and reverse faulting for $\mathrm{L}_{\mathrm{T}}=50,100$, and $150 \mathrm{~km}$, respectively. Grey dots illustrate boundary element grid points where stresses were calculated. Model dimensions are $X_{0}=240 \mathrm{~km}, Y_{o}=160 \mathrm{~km}$, and $Z_{0}=15 \mathrm{~km}$, with $\chi=0.05$ and a far-field displacement $U_{0}=100 \mathrm{~m}$. Note that all ridge axes have been shifted to $X=0 \mathrm{~km}$. (b) Comparison of regions of strike-slip and reverse faulting as a function of plate thickness. Red, blue, and green contours illustrate the cases with plate thickness of 5,15 , and $25 \mathrm{~km}$, respectively. Transform length is $100 \mathrm{~km}$, all other model parameters are the same as (a).

between the stress fields surrounding the ridge-transform intersections at either end of the transform. However, for transform with offsets $\geq 100 \mathrm{~km}$ there is little interaction and the pattern of faulting at the end of each segment remains relatively constant with increasing offset lengths.

\section{Plate Thickness}

In order to assess the sensitivity of our numerical results to plate thickness, we examine the predicted pattern of faulting for vertical model dimensions, $Z_{0}$, of 5,15 , and $25 \mathrm{~km}$ (Figure 5-8). In this context, the thickness of the plate is defined by the depth to the zero shear stress condition at the base of the model domain. As plate thickness increases, the area adjacent to the ridge-transform intersection characterized by strike-slip and reverse faulting is predicted to grow, and the calculated asymmetry in $\tau_{\mathrm{dev}} / \sigma_{\mathrm{dev}+\text { lith }}$ across the segment ends 
is enhanced (Figure 5-7b). These changes are caused by the greater coupling area, and thus increased shear stresses, associated with a thicker plate. Note that the changes in fault style due to variations in plate thickness (Figure 5-8) are significantly smaller than those associated with variations in the coupling parameter (Figure 5-5). The results in Figure 5-8 show that even for $Z_{0}=5 \mathrm{~km}, \chi$ is unlikely to be significantly greater than 0.05 .

In reality, the zero shear stress region (which can approximated by the brittle/ductile transition zone) is likely to increase with crustal age. Thus, solutions for a thin plate (Figure 5-8a) are more applicable to the ridge-axis region, while those for a thicker plate (Figures $5-8 b$ and $5-8 c)$ are more appropriate for off-axis regions. Due to technical complexities, we have not yet obtained solutions in which the brittle/ductile transition depth increases with crustal age. This issue will be addressed in future numerical experiments.

\subsubsection{Transform Strength and Stress State at Inside Corners}

As illustrated by Phipps Morgan and Parmentier [1984] and Grindlay and Fox [1993], the predicted pattern of faulting depends primarily on the relative magnitude of the far-field extensional stress to the shear stress along the transform. Increasing the coupling parameter or the thickness of the plate tends to enhance the shear stresses along the transform and promote strike-slip faulting throughout the model space. In contrast, increasing the far-field stress through mechanisms such as enhanced amagmatic spreading tends to promote ridgeparallel normal faulting. Thus, by comparing our model predictions with observations of faulting and seismicity from mid-oceans ridges, we can constrain the relative magnitude of these competing effects.

Specifically, our numerical results clearly show that oceanic transform faults are zones of significant weakness. Observed patterns of faulting and seismicity are consistent with only $\sim 5 \%$ of the total shear strain being coupled across the transform (i.e., $\chi=0.05$ ). This implies that the additional $95 \%$ of the shear strain must dissipate rapidly, either through aseismic creep or slow earthquakes. Calculations of seismic deficits along oceanic transform faults also suggest a significant amount of aseismic slip. For example, Okal and 

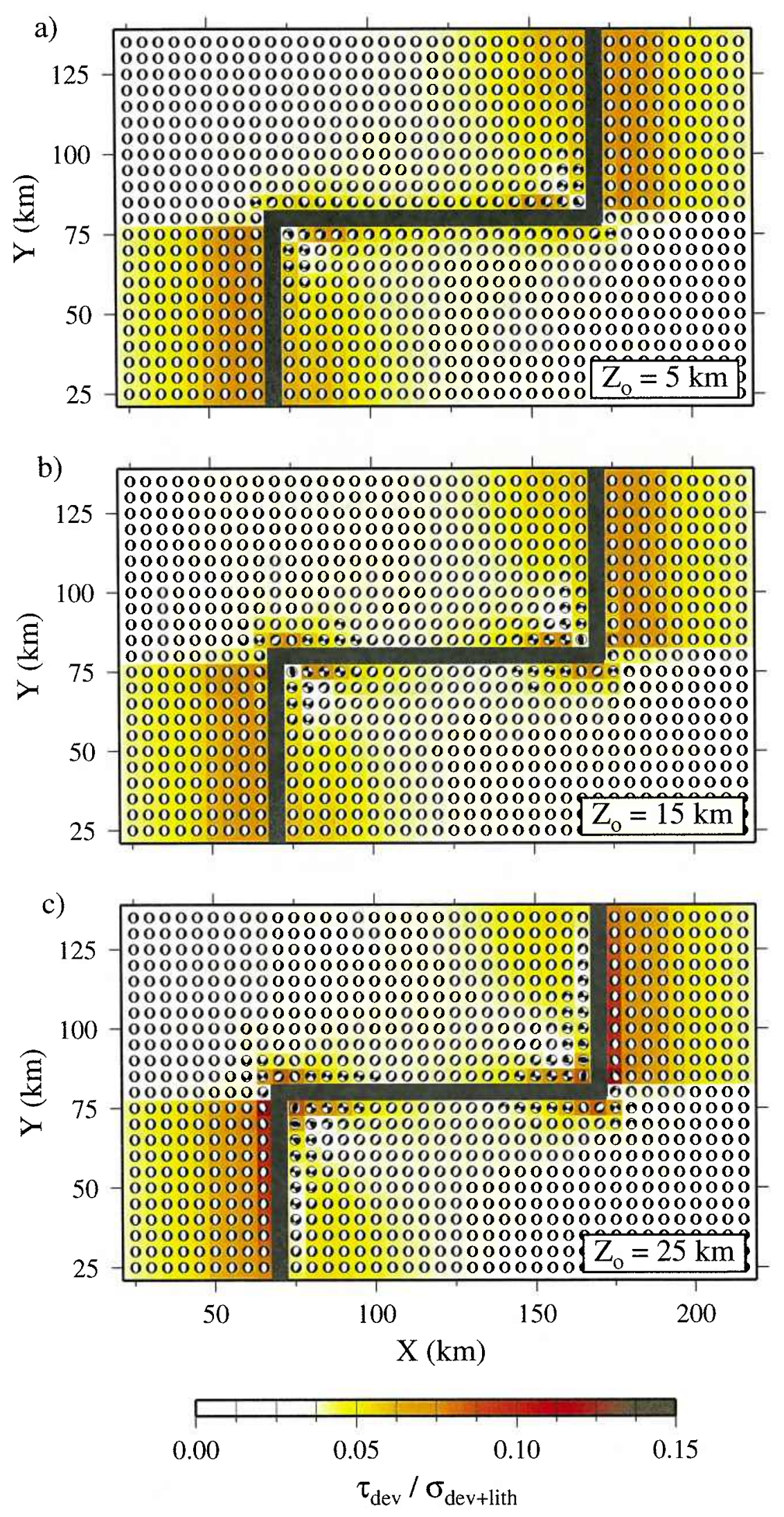

144 
Figure 5-8: (facing page) Effect of plate thickness on the style of faulting near an oceanic transform fault. Orientations of optimal fault planes for plate thickness of (a) $5 \mathrm{~km}$, (b) 15 $\mathrm{km}$, and (c) $25 \mathrm{~km}$, with $\chi=0.05$ and a far-field displacement $\mathrm{U}_{\mathrm{o}}=100 \mathrm{~m}$. Horizontal model dimensions are $X_{0}=240 \mathrm{~km}$ and $Y_{0}=160 \mathrm{~km}$. Thick grey lines show location of ridge segments and transform fault. Color shading indicates the relative magnitude of $\tau_{\mathrm{dev}} / \sigma_{\mathrm{dev}+\text { lith }}$. Note that fault style changes only slightly with increasing plate thickness.

Langenhorst [2000] and Boettcher and Jordan [2001] found that seismic moment release can account for only $10-15 \%$ of the total slip predicted from kinematic models on most oceanic transforms. These observations are in contrast to continental environments, where studies have found little evidence for seismic deficits on major stike-slip faults [Brune, 1968; Kiratzi, 1993; Stein and Hanks, 1998]. Thus, it appears that oceanic transform faults are characterized by greater amounts of aseismic slip than continental strike-slip faults.

Another important, and somewhat surprising, prediction of our calculations is that ICs are not characterized by elevated extensional stresses relative to OCs. Instead, the opposite effect is predicted, with shear along the transform suppressing ridge-normal extension at ICs. This prediction indicates that the large throw commonly observed on IC faults and the enhanced microseismicity at ICs may not be caused by elevated extensional stresses in these regions. In the following section we propose an alternative mechanism to explain the growth of large normal faults at ICs based on the geometry of a ridge-transform intersection.

\subsection{Development of Normal Faults and Axial Topography at a Ridge-Transform Intersection}

A number of different mechanisms have been proposed for the development of asymmetric axial topography near ridge-transform intersections. Chen [1989] suggested that IC uplift is caused by a "twisting moment" generated by increasing frictional stresses with depth along an active transform fault. Bercovici et al. [1992] showed that in a non-linear visco-elastic rheology purely strike-slip motion can result in uplifted transverse ridges adjacent to the 
transform. Alternatively, Chen and Lin [1999] suggested that the flexural response of the lithosphere to the unloading of deep axial and transform valleys may result in preferential uplift at ICs. A final class of models that have been proposed to explain the asymmetric ICOC topography deal with the kinematic differences between the active and inactive sections of a transform fault [Kuo et al., 1984; Severinghaus and Macdonald, 1988; Escartín and Lin, 1995]. These models hypothesize that the active, and thus weak, portion of a transform acts as a free boundary, resulting in preferential uplift and faulting in the decoupled IC crust. However, to date no quantitative studies have been conducted to test the effect of a "weak" transform on the growth of neighboring normal faults.

\subsubsection{Model Setup}

We investigate normal fault development at the end of a spreading segment using a simple 3-D boundary element model of a graben adjacent to a transform fault. The model consists of two inward dipping normal faults with a surface separation distance $S$, placed at a distance D from a transform (Figure 5-9). The normal faults are characterized by an along-strike length, $\mathrm{L}_{\mathrm{F}}$, a down-dip width, $\mathrm{W}_{\mathrm{F}}$, and a dip angle, $\theta$. The transform extends outward a distance of $100 \mathrm{~km}$ from the center of the graben and down-dip to a depth of $2 \mathrm{~W}_{\mathrm{F}}$. Deformation is driven by a uniform far-field strain, $\varepsilon_{\mathrm{f} . f .}$, acting in the $\mathrm{x}$-direction. The magnitude of $\varepsilon_{\text {f.f. }}$ is set to $10^{-5}$ throughout this study, resulting in a far-field tensile stress of $\sim 0.8 \mathrm{MPa}$ for a homogenous elastic halfspace with Young's modulus $\mathrm{E}=70 \mathrm{GPa}$ and Poisson's ratio $\nu=0.25$. We note that the magnitude of the deformation predicted by our model scales linearly with the far-field strain. Calculations are performed for both an infinite elastic halfspace and a plate thickness of $10 \mathrm{~km}$. For the $10-\mathrm{km}$ thick plate, the basal boundary is set to be shear stress free with zero vertical displacement.

The normal and transform fault planes are discretized into $1 \mathrm{~km} \times 1 \mathrm{~km}$ subelements, defined to have zero shear stress and no normal displacement. This formulation assumes the faults behave as frictionless surfaces, resulting in the maximum predicted slip for a given 


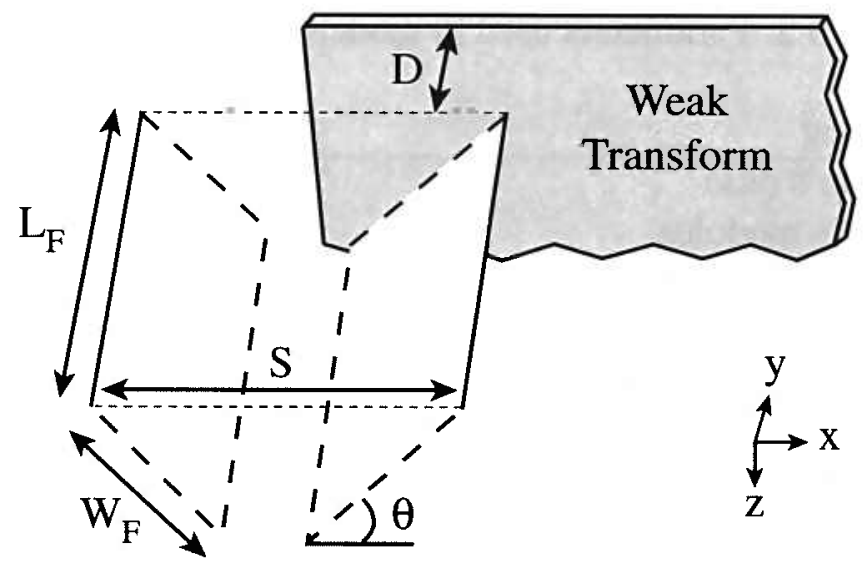

Figure 5-9: Model setup for boundary element analysis of normal fault development near a transform. Two inward dipping normal faults with a surface separation, $\mathrm{S}=15 \mathrm{~km}$, are placed a distance $\mathrm{D}$ from a transform. Normal faults are characterized by along-strike length, $\mathrm{L}_{\mathrm{F}}=20 \mathrm{~km}$, a down-dip width, $\mathrm{W}_{\mathrm{F}}=10 \mathrm{~km}$, and a dip angle, $\theta=45^{\circ}$. Boundary conditions on all fault planes are defined as zero shear stress with no normal displacement. Deformation is driven by a far-field strain, $\varepsilon_{\text {f.f. }}$, in the $\mathrm{x}$-direction. All calculations were performed with a Young's modulus $\mathrm{E}=70 \mathrm{GPa}$ and a Poisson's ratio $\nu=0.25$.

far-field strain. While this assumption is clearly an over-simplification, it results in normal faults with dip angles of $45^{\circ}$, consistent with observations of focal mechanisms at oceanic ridges [Thatcher and Hill, 1995]. A frictionless fault also represents an end-member type of fault behavoir that has been used to highlight the interaction of normal faults in previous numerical studies [e.g., Crider and Pollard, 1998]. The far-field tensile stresses generate a component of opening across the faults. However, except at very shallow depths these deviatoric stresses will not be sufficient to overcome the lithostatic pressure. Thus, because gravitational forces are not modeled explicitly in our numerical simulations, the condition of no normal displacement is imposed to simulated the lithostatic pressure acting on the fault planes [e.g., Rudnicki and $W u, 1995]$.

\subsubsection{Numerical Results}

Using the numerical approach described above, we compare the surface deformation associated with a graben adjacent to a transform to the deformation predicted when no transform 
Table 5.2: Parameters used in models of fault development.

\begin{tabular}{llll} 
Symbol & Meaning & Value & Units \\
\hline$\nu$ & Poisson's ratio & 0.25 & \\
$\mathrm{E}$ & Young's modulus & 70 & $\mathrm{GPa}$ \\
$\mathrm{L}_{\mathrm{F}}$ & along-strike length of normal fault & 20 & $\mathrm{~km}$ \\
$\mathrm{~W}_{\mathrm{F}}$ & down-dip width of normal fault & 10 & $\mathrm{~km}$ \\
$\theta$ & dip angle of normal faults & $45^{\circ}$ & \\
$\mathrm{S}$ & surface separation distance between normal faults & 15 & $\mathrm{~km}$ \\
$\mathrm{R}$ & x-offset distance between grabens & $0-22.5$ & $\mathrm{~km}$ \\
$\mathrm{D}$ & y-offset distance between graben and transform & $0-5$ & $\mathrm{~km}$ \\
$\varepsilon_{\mathrm{f.f}}$ & far-field strain & $10^{-5}$ & \\
$\mathrm{Z}_{\mathrm{o}}$ & plate thickness & $10, \infty$ & $\mathrm{km}$ \\
\hline
\end{tabular}

is present. For the case of no tranform fault, the surface deformation is symmetric across the graben, with maximum flank uplift at the center of both faults (Figure 5-10a). In contrast, the presence of a transform fault generates a highly asymmetric pattern of surface deformation, with enhanced uplift on the IC fault adjacent to the transform (Figure 5-10b). This preferential uplift is associated with the formation of a neighboring topographic low at the intersection of the IC fault and the transform. There is also slightly enhanced uplift at the center of the OC fault, although the magnitude is significantly smaller than at the IC.

The distribution of slip on the IC and OC fault planes is shown in Figure 5-11. The OC fault is characterized by a symmetric distribution of dip-slip motion, with the maximum slip occurring near the surface at the center of the fault. In contrast, dip-slip motion on the IC fault is skewed, with greater slip towards the end of the fault adjacent to the transform. Both the IC and OC faults are characterized by a small component of strike-slip motion. This prediction is consistent with models by Crider and Pollard [1998], who observed that normal faults under uni-axial tension will experience strike-slip displacement due to their shape and interaction with adjacent faults.

The enhanced slip on the IC fault near the transform generates an asymmetric distribution of throw along the fault (Figure 5-12). Fault throw is a useful parameter because it can be easily measured in the field using a combination of bathymetry and side-scan sonar data. 

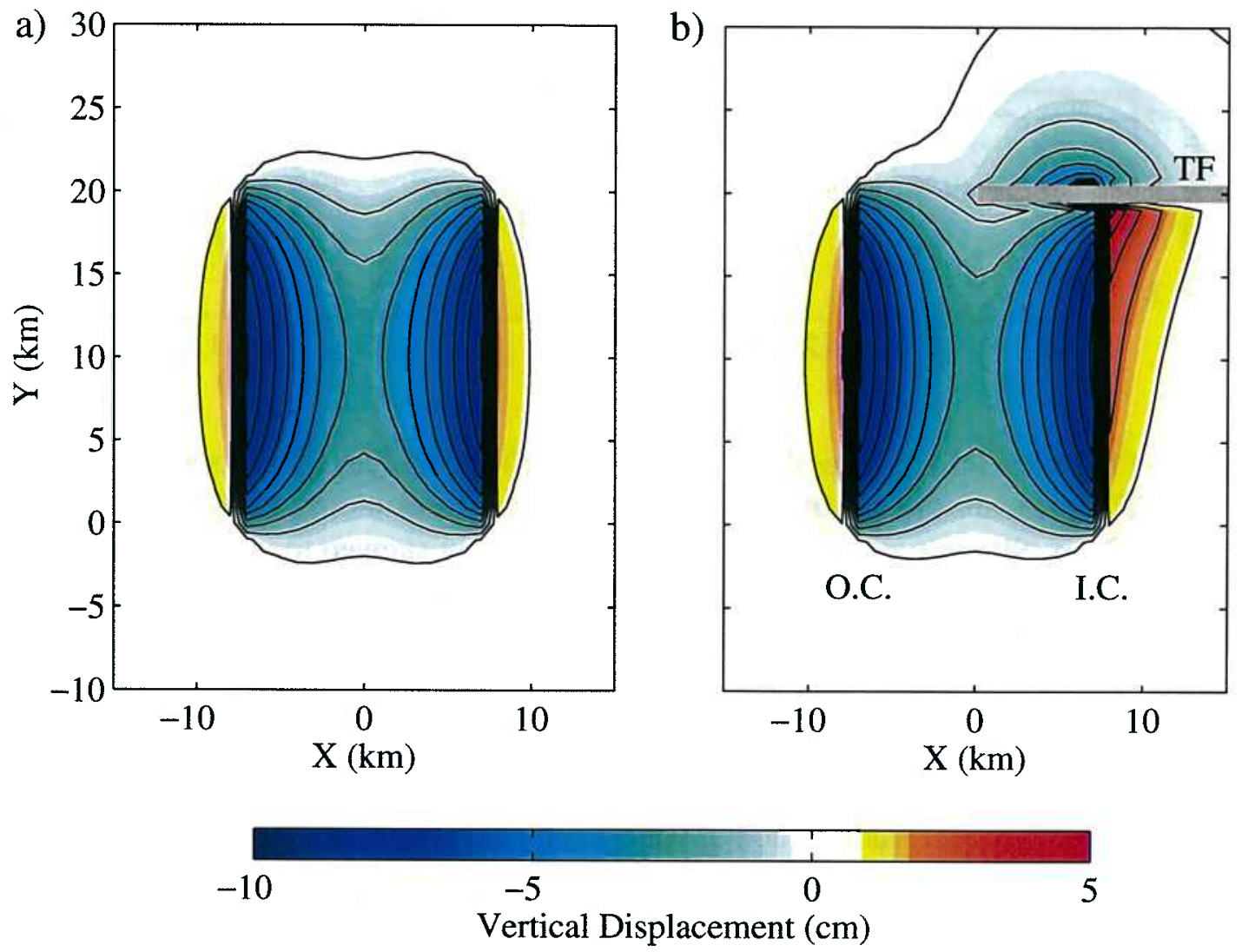

Figure 5-10: (a) Vertical displacement at the surface of an elastic halfspace for two inward dipping normal faults with no transform present. Red and blue shades represent positive (upward), and negative (downward) displacements, respectively. Faults have dimensions $\mathrm{L}_{\mathrm{F}}=20 \mathrm{~km}$ and $\mathrm{W}_{\mathrm{F}}=10 \mathrm{~km}$, and a dip angle, $\theta=45^{\circ}$. Deformation is driven by a farfield strain, $\varepsilon_{\text {f.f. }}=10^{-5}$. (b) Vertical surface displacement for two inward dipping normal faults adjacent to a transform. Graben is offset a distance, $\mathrm{D}=0 \mathrm{~km}$, from the transform. Thick grey line illustrates the location of the transform fault (TF), and inside- (I.C.) and outside-corner (O.C.) faults are marked. Note the enhanced uplift on the IC fault adjacent to the transform. 

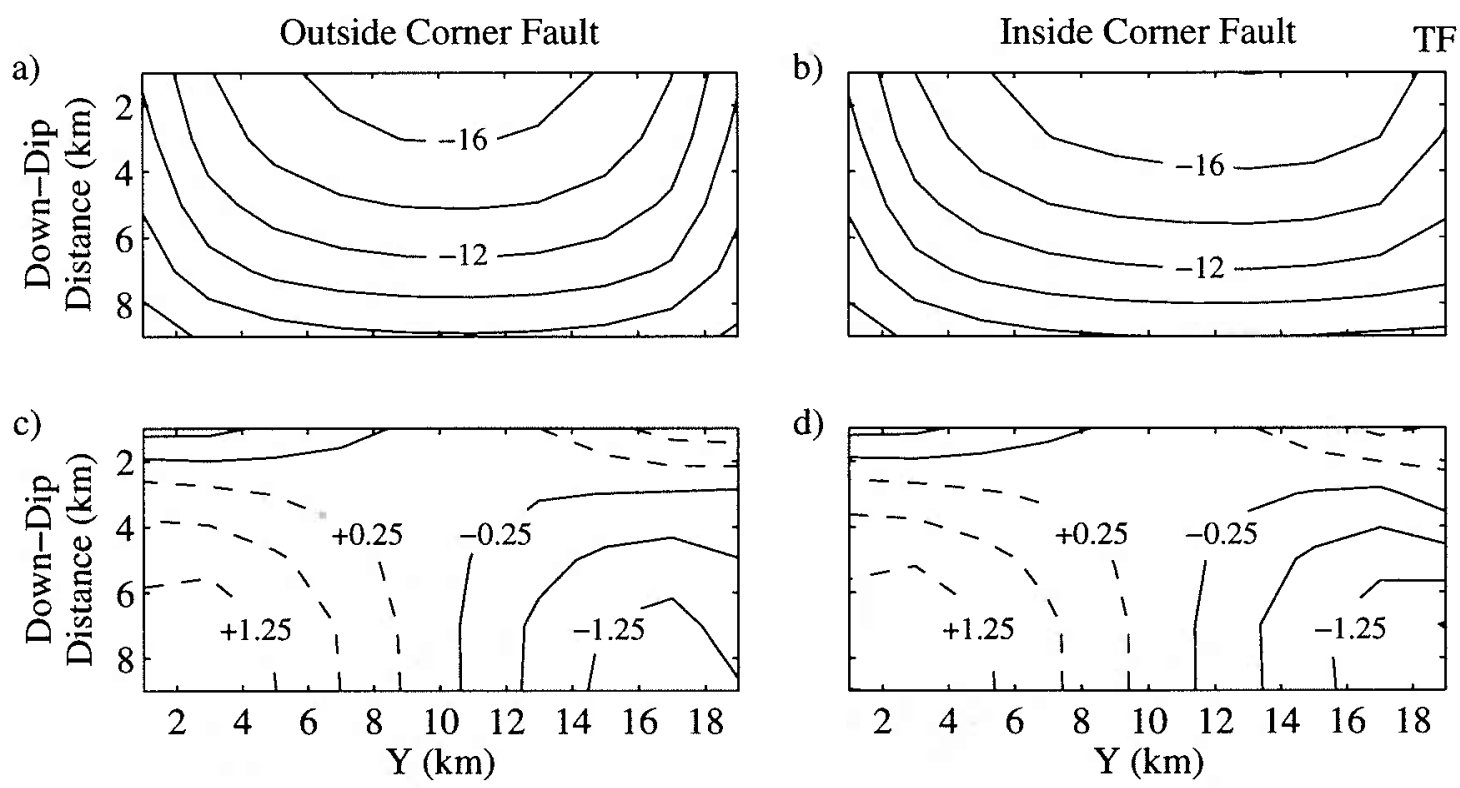

d)

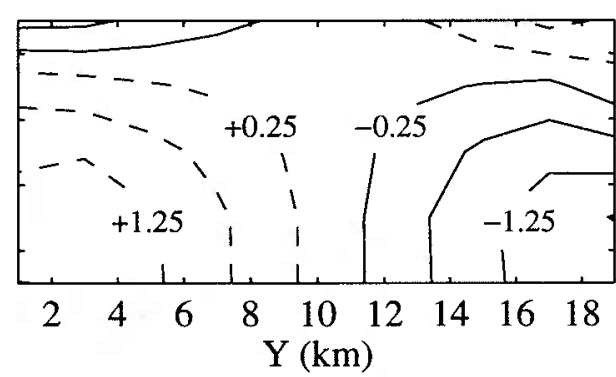

Figure 5-11: Distribution of dip-slip motion on the (a) OC and (b) IC faults shown in Figure $5-10 \mathrm{~b}$. TF represents the relative location of the transform along the IC fault. Contour interval is $2 \mathrm{~cm}$. Slip is symmetric along the $\mathrm{OC}$ fault, but skewed to larger values near the transform on the IC fault. (c,d) Distribution of strike-slip motion along OC and IC faults. Contour interval is $0.5 \mathrm{~cm}$.

Furthermore, fault throw is related to only the behavior of the fault and the effects of slope failure, while other observables such as topography are the integrated result of many different processes (e.g., fault growth, thermal subsidence, isostatic compensation of crustal thickness variations, and slope failure). Because the magnitude of the fault throw scales linear with the far-field strain, we simplify our analysis through normalizing the calculated throw by the maximum throw in the absence of any transform influence.

In Figure 5-12 we evaluate the sensitivity of the throw on the IC and OC faults to the offset distance, D, separating the transform from the graben. In all cases, throw on the IC fault is greatest at a distance of $2-3 \mathrm{~km}$ from the transform $(\mathrm{Y}=17-18 \mathrm{~km})$. Maximum throw is predicted for $\mathrm{D}=0 \mathrm{~km}$, in which case the throw on the IC fault is enhanced more than $20 \%$ relative to the case with no transform. As offset distance increases, the effect of the transform diminishes. The calculated throw on the OC fault is only slightly enhanced for all offsets, with a maximum increase of only $\sim 3 \%$ for $D=0 \mathrm{~km}$. 

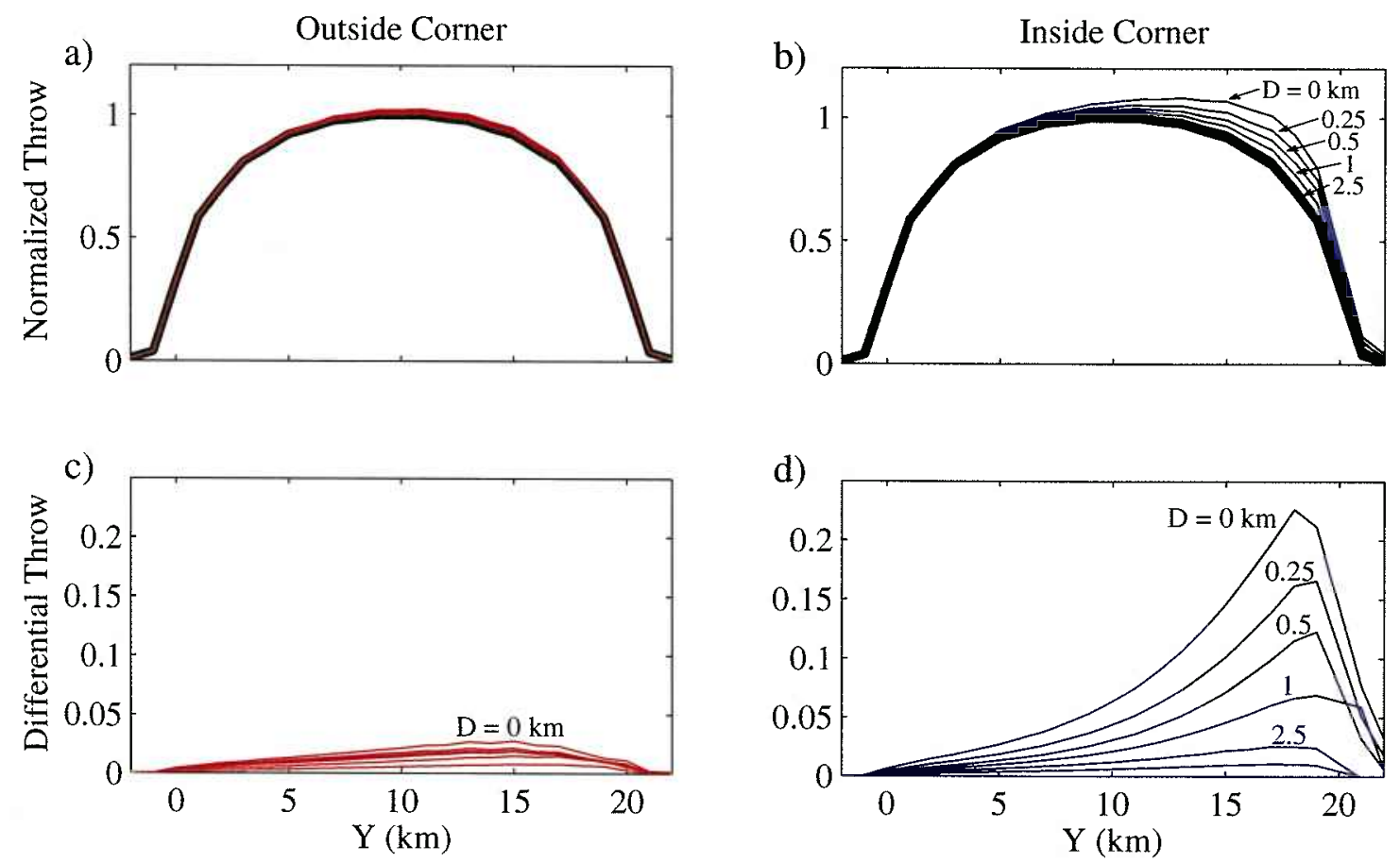

Figure 5-12: Normalized throw along the (a) OC and (b) IC faults. Throw is normalized to the maximum value for the case with no transform (thick black curve). Red (OC) and blue (IC) curves illustrate throw for offsets, D, ranging from 0-5 km. (c,d) Differential throw along the $\mathrm{OC}$ and IC faults, respectively. Differential throw is defined as the difference in throw between the case with a transform present and the case without a transform fault. Calculations are performed in an elastic halfspace. Note that throw on the IC fault is preferentially enhanced in the $5 \mathrm{~km}$ nearest the transform. 

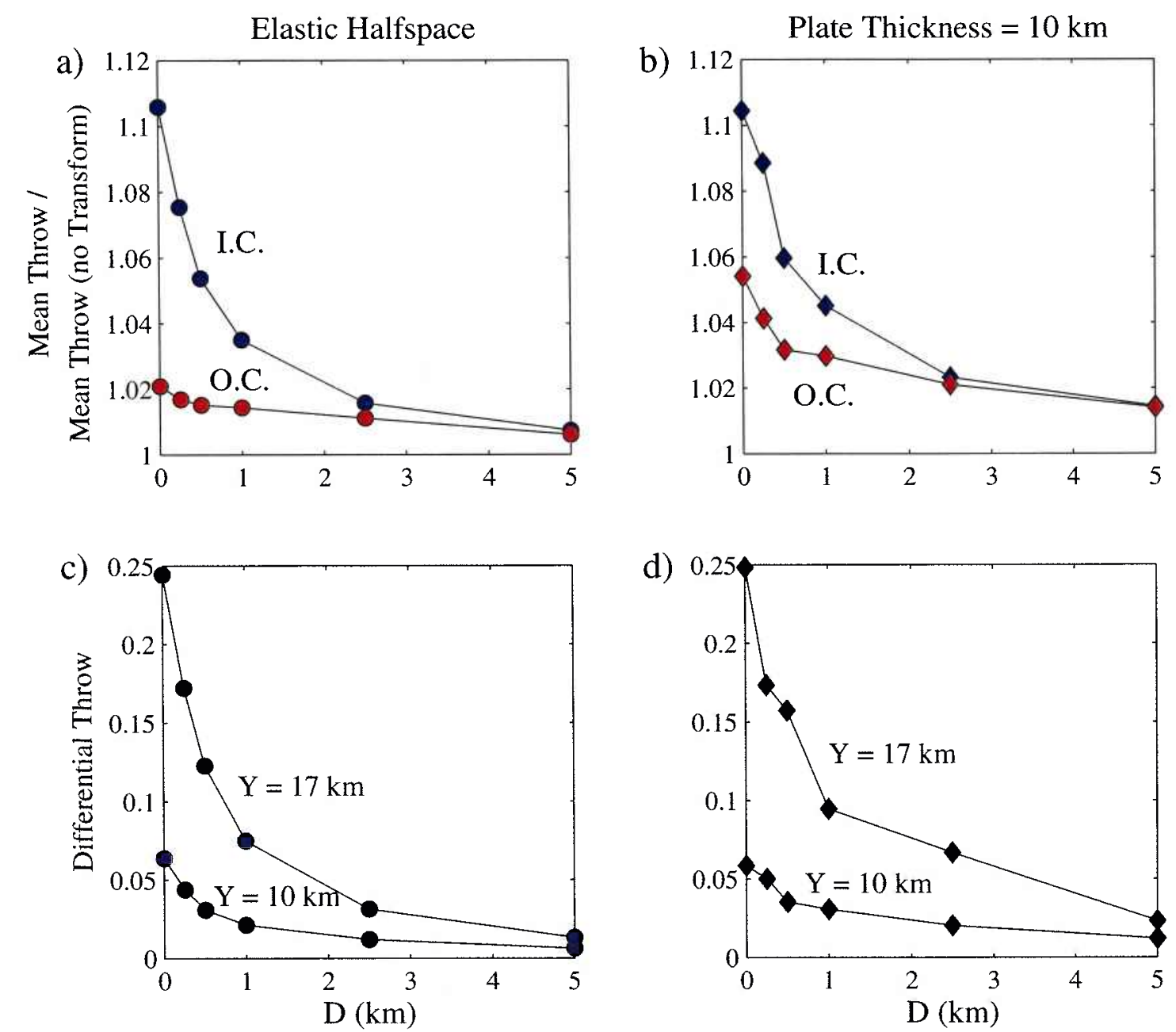

Figure 5-13: Mean fault throw as a function of the offset, D, between the transform and graben for (a) an elastic halfspace and (b) a plate thickness of $10 \mathrm{~km}$. Red and blue symbols illustrate the results of individual numerical experiments for the OC and IC faults, respectively. Mean throw is normalized to throw in the absence of a transform fault. $(c, d)$ Differential throw measured at $Y=10$ and $17 \mathrm{~km}$ as a function of offset for the elastic halfspace and $10-\mathrm{km}$ plate, respectively. Note that results of the elastic halfspace and the $10-\mathrm{km}$ plate are similar, though the $10-\mathrm{km}$ plate tends to generate slightly elevated values of mean throw. 
The effect of a weak transform on the development of neighboring normal faults is summarized in Figure 5-13. As illustrated in Figures 5-12b and 5-12d, both the mean and differential throw on the IC fault are calculated to decrease rapidly with increasing offset distance in an elastic halfspace. Similar variations are predicted for a plate thickness of $10 \mathrm{~km}$ (Figure 5-13b), though the $10-\mathrm{km}$ plate tends to generate slightly elevated values of mean throw relative to the elastic halfspace. Figures 5-13c and 5-13d illustrate the asymmetry in the distribution of throw along the IC fault. For example, with $\mathrm{D}=0 \mathrm{~km}$, throw at $\mathrm{Y}=17 \mathrm{~km}$ is increased $\sim 25 \%$ relative to the case with no transform, while at the center of the fault $(\mathrm{Y}=10 \mathrm{~km})$ throw increases by only $\sim 5 \%$. In summary, these results indicate that a normal fault forming at the IC of a ridge transform intersection will experience preferential uplift relative to normal fault forming on the OC. Moreover, a specific IC fault will be characterized by an asymmetric along-strike distribution of throw, with enhanced uplift adjacent to the transform.

Throughout these calculations, we have characterized the transform fault as a weak vertical plane sustaining zero shear stress. In reality, it is unlikely that the transform will behave in a stress-free manner on the time scale of an individual earthquake. Rather, we hypothesize that enhanced uplift at an inside corner will be accommodated in one of two ways. In the first senario, vertical stresses that accumulate along the normal fault after a slip event will be preferentially released through aseismic slip along the transform. This possibility is supported by the calculations presented in Section 5.2 and seismic observations [Okal and Langenhorst, 2000; Boettcher and Jordan, 2001], which indicate that oceanic transform faults are characterized by significant amounts of aseismic slip. Alternatively, over geologic time, stresses may be preferentially released at the segment end through larger and/or more frequent slip events. This model could provide an explanation for the elevated levels of microseismicity at ICs [Rowlett and Forsyth, 1984; Wolfe et al., 1995; Barclay et al., 2001] even though the results of Section 5.2 show these areas are not predicted to have elevated extensional stresses. Thus, while individual normal faulting events may have symmetric distributions of throw, the long term development of the IC fault may behave in the manner predicted by our simple model. 


\subsubsection{A Model for Enhanced Fault Growth at Inside-Corners}

Observations from slow-spreading ridges have shown that normal faults forming at the IC of a ridge-transform intersection are characterized by greater throw than faults forming on the OC [Severinghaus and Macdonald, 1988; Tucholke and Lin, 1994; Allerton et al., 1995; Escartín et al., 1999]. Moreover, many of IC faults are observed to have highly asymmetric scarp shapes, with greatest throw near the end of the segment. Based on the numerical results presented in this study we propose a simple model for enhanced normal fault development at the IC of a ridge-transform intersection. Our model assumes that over time scales longer than a typical earthquake cycle, transform faults will act as zones of significant weakness. Normal faults that develop at the IC will feel this weak surface, and slip will preferentially accumulate toward the end of the fault approaching the transform. Over time, this will result in an additional feedback, as the portion of the fault with a larger surface area will tend to accumulate more slip for each increment of far-field strain. In constrast, on the $\mathrm{OC}$, where we assume the inactive transform to be locked, fault growth will not be characterized by enhanced slip toward the segment end.

This model does not require that each individual earthquake be characterized by an asymmetrical distribution of throw. Rather over geologic time, faults on the IC will preferentially release stress near the transform. This stress release may be accommodated by more frequent slip events near the segment end, possibly explaining the elevated levels of microseismicity in these regions [e.g., Rowlett and Forsyth, 1984; Wolfe et al., 1995; Barclay et al., 2001]. Alternatively, vertical stresses that accumulated near the segment end after a slip event may be released slowly through aseismic creep on the transform. While this simple model cannot explain all of the complexities observed at ICs, it does provide a plausible mechanism to explain the enhanced growth of normal faults, which contribute to the formation of elevated topography at a ridge-transform intersection.

\subsection{Conclusions}

In this study we have used 3-D boundary element calculations to quantify the effects of transform faults on stress state, fault development, and morphology at oceanic spreading 
centers. We show that the predicted pattern of stress and fault formation near a oceanic transform fault is controlled by the relative magnitude of the far-field extensional stresses to the shear stresses along the transform. Our numerical results suggest that over time scales longer than a typical earthquake cycle (i.e., $\sim 100$ years), oceanic transform faults behave as zones of significant weakness. Observations of faulting and seismicity near ridge-transform intersections are most consistent with $\sim 5 \%$ mechanical coupling across the transform (i.e., coupling coefficient $\chi=0.05$ ). On time scales shorter than a typical earthquake cycle, coupling along transforms may experience significant temporal variations. Specifically, we hypothesize that transient "locked" periods may explain the presence of anomalous reverse faulting mechanisms observed at inside-corners. Another important prediction of our modeling is that inside-corners are not characterized by enhanced extensional stresses relative to outside-corners. Instead, the opposite effect occurs, with shear stresses along the transform suppressing ridge-normal extension at inside-corners. This indicates that an alternative mechanism is necessary to explain the enhanced normal fault growth and microseismicity observed in these regions.

We propose that such a mechanism involves the interaction of normal faults near the end of a spreading-segment with the neighboring transform fault. Model results show that normal faults that develop at an inside-corner, adjacent to a weak transform, experience preferential growth relative to faults forming on the outside corner away from the transform. We note that individual earthquakes may not be characterized by asymmetric patterns of throw. Rather, we hypothesize that enhanced fault growth may be manifest in either stress relaxation through aseismic uplift along a transform, or more frequent slip events toward the end of the fault adjacent to the transform. Finally, we note that while this simple model cannot explain all the complexities of fault dynamics at a ridge-transform intersection, it does present a plausible model for enhanced fault growth and fault-related topography at inside-corners. 


\title{
Chapter 6
}

\section{Relationship between Seismic Velocity}

\section{and Igneous Rock Composition}

\begin{abstract}
This study presents a new method to quantitatively assess the relationship between the composition and seismic velocity of igneous rocks. We perform thermodynamic calculations of the equilibrating phase assemblages predicted for all igneous composition space at various pressure and temperature conditions. Seismic velocities for each assemblage then are estimated from mixing theory using laboratory measurements of the elastic parameters for pure mineral phases. The resultant velocities are used to derive a direct relationship between $V_{p}$ and major element composition valid to $\pm 0.12 \mathrm{~km} / \mathrm{s}$ for pressure and temperature conditions along a normal crustal geotherm in the depth range of 5-50 km. Finally, we use the calculated velocities to invert for major element chemistry as a function of seismic velocity assuming only the in situ temperature and pressure conditions are known. Compiling typical velocity-depth profiles for the lower continental and oceanic crust, we calculate compositional bounds for each of these geologic environments. We find that the acceptable compositional range for the lower continental crust is extremely large, ranging from basaltic to granitic compositions, and conclude that seismic velocity measurements alone are insufficient to provide fundamental constraints on the composition of the lower continental crust. In the case of oceanic crust, observed lower crustal velocities are $0.2-0.3$ $\mathrm{km} / \mathrm{s}$ lower than velocities calculated based on the average bulk composition of gabbros in drill cores and exposed ophiolite sequences. We attribute this discrepancy to residual porosity at crustal depths less than $\sim 10 \mathrm{~km}$, and suggest caution when inferring mantle melting parameters from observed velocities in the lower oceanic crust.
\end{abstract}




\subsection{Introduction}

Knowledge of the composition of the lower continental and oceanic crust is critical to our understanding of crustal formation and evolution. The composition of the crust also affects crustal rheology and plays an important role in controlling active tectonic processes in continental and oceanic environments. However, to date the composition of the lower crust remains poorly constrained. Unlike the upper crust, which is easily accessible to geologic sampling, direct sampling of the lower crust is limited to xenoliths, high-grade metamorphic terranes, and, in the case of oceanic crust, ophiolites. Although these samples have provided valuable insights into the composition of the lower crust, their heterogeneity and possible sampling bias make it difficult to assess how representative they are of the overall composition of the lower crust.

An alternate approach to studying lower crustal rocks involves seismic measurements, which allow large sections of the lower crust to be characterized in situ. Unfortunately, this method has been limited by the difficulty in determining rock types from estimates of average seismic velocity. One method that is frequently used to extrapolate composition from seismic data is forward modeling of seismic velocities for rocks with known seismic properties [e.g., Pakiser and Robinson, 1966; Holbrook et al., 1992; Christensen and Mooney, 1995; Rudnick and Fountain, 1995]. In this approach, the elastic properties of a wide range of crustal compositions are first measured in the laboratory at different temperatures and pressures [e.g., Birch, 1960, 1961; Christensen, 1965, and numerous studies thereafter]. These results are then extrapolated to geologic conditions and compared with seismic refraction data to determine which rock types are consistent with the observed seismic data. While this method can be used to qualitatively assess the composition of the lower crust, forward modeling cannot quantitatively evaluate the full range of compositions that are consistent with a specific set of seismic observations.

Kelemen and Holbrook [1995] conducted an early attempt to directly relate the chemical compositional of igneous rocks to their seismic velocity. Compiling laboratory data 
on samples for which both seismic P-wave velocity, $V_{p}$, and composition data were available, Kelemen and Holbrook [1995] used multiple linear regression to relate $V_{p}$ to $\mathrm{SiO}_{2}$ and $\mathrm{MgO}$. The utility of the resulting empirical relationship, however, is limited by a number of factors. First, the samples for which both $V_{p}$ and compositional data are available do not encompass the full spectrum of compositions that may be present in the lower crust. Second, laboratory measurements are hampered by microcracks and secondary alteration that may not be present in situ. Finally, the pressure and temperature conditions at which a bulk composition equilibrates will govern its crystallizing mineral assemblage. Thus, for any single bulk composition, a range of seismic velocities will be predicted as a function of the equilibrium conditions [Sobolev and Babeyko, 1994]. The presence of garnet is of particular importance because its high seismic velocity implies that even in small quantities it can significantly increase the overall seismic velocity of a crystallizing assemblage. Because Kelemen and Holbrook [1995] were interested in studying igneous crust outside the garnet stability field, they eliminated samples containing garnet from their analysis. However, a comprehensive estimate of the composition of the lower crust from seismic data must incorporate garnet and other high pressure phases.

In this study we present a new method to quantitatively assess the relationship between the composition and seismic velocity of igneous rocks. Specifically, we assess the full range of igneous compositions that are consistent with a particular seismic velocity, assuming that only the in situ temperature and pressure conditions are known. We perform thermodynamic calculations to determine the crystallizing mineral assemblages at various equilibrium conditions for all igneous composition space. Seismic velocities for each assemblage are estimated from mixing theory using laboratory measurements of the elastic parameters for pure mineral phases. The resultant velocities are then used to derive a direct relationship between $V_{p}$ and major element oxide composition, and the implications of this relationship for mantle melting systematics are explored. Finally, we use the calculated velocities to invert directly for major element chemistry as a function of $V_{p}$ and discuss the implications for the lower continental and oceanic crust. 
Table 6.1: Variance-covariance matrix for 7 major element oxides in IGBA database. Only the lower half of the symmetric matrix is shown. Note that the units of variance are $\mathrm{wt} \%{ }^{2}$ and the total variance of the system is defined by the trace of the variance-covariance matrix (total variance $=155.51)$

\begin{tabular}{lccccccc} 
& \multicolumn{7}{c}{ Oxide } \\
\cline { 2 - 8 } Oxide & $\mathrm{SiO}_{2}$ & $\mathrm{Al}_{2} \mathrm{O}_{3}$ & $\mathrm{MgO}$ & $\mathrm{FeO}^{*}$ & $\mathrm{CaO}$ & $\mathrm{Na} 2 \mathrm{O}$ & $\mathrm{K}_{2} \mathrm{O}$ \\
\hline $\mathrm{SiO}_{2}$ & 82.09 & & & & & & \\
$\mathrm{Al}_{2} \mathrm{O}_{3}$ & 2.67 & 10.77 & & & & & \\
$\mathrm{MgO}$ & -30.03 & -10.74 & 30.59 & & & & \\
$\mathrm{FeO}^{*}$ & -25.69 & -2.58 & 8.53 & 12.89 & & & \\
$\mathrm{CaO}$ & -25.66 & 0.60 & 6.76 & 8.51 & 13.95 & & \\
$\mathrm{Na}_{2} \mathrm{O}$ & 4.44 & 2.02 & -4.50 & -2.15 & -2.61 & 2.42 & \\
$\mathrm{~K}_{2} \mathrm{O}$ & 7.64 & 0.42 & -4.17 & -3.34 & -3.94 & 1.07 & 2.80 \\
\hline
\end{tabular}

\subsection{Igneous Composition Space}

We characterize the composition space of naturally occurring igneous rocks using data from the IGBA database [Chayes, 1985; Brandle and Nagy, 1995]. IGBA contains $~ 19,000$ volcanic and plutonic rock samples compiled from over 1,300 sources across a broad spectrum of geologic environments. Of these samples, 18,637 include analyses on the 7 major element oxides, $\mathrm{SiO}_{2}, \mathrm{Al}_{2} \mathrm{O}_{3}, \mathrm{MgO}, \mathrm{FeO}^{*}\left(\mathrm{FeO}+0.9 \mathrm{Fe}_{2} \mathrm{O}_{3}\right), \mathrm{CaO}, \mathrm{Na}_{2} \mathrm{O}$, and $\mathrm{K}_{2} \mathrm{O}$. The variance-covariance matrix for the 7 major oxides is calculated in Table 6.1. The total variance of the oxide space is defined by the trace of the matrix, and the relative proportion explained by each oxide can be calculated by dividing the individual variance by the total variance. For example, $\mathrm{SiO}_{2}$ and $\mathrm{MgO}$ account for the largest share of the total variance, explaining $53 \%$ and $20 \%$, respectively.

To further examine the structure of igneous composition space, we perform a principal component analysis on the 7 major element oxides in the IGBA database. The principal components of a system correspond to the eigenvectors of the variance-covariance matrix, and represent the coordinate axes that most efficiently explain the total variance of the system. The associated eigenvalues give the portion of the total variance explained by each eigenvector. The principal components of the IGBA oxide data are given in Table 6.2 and 

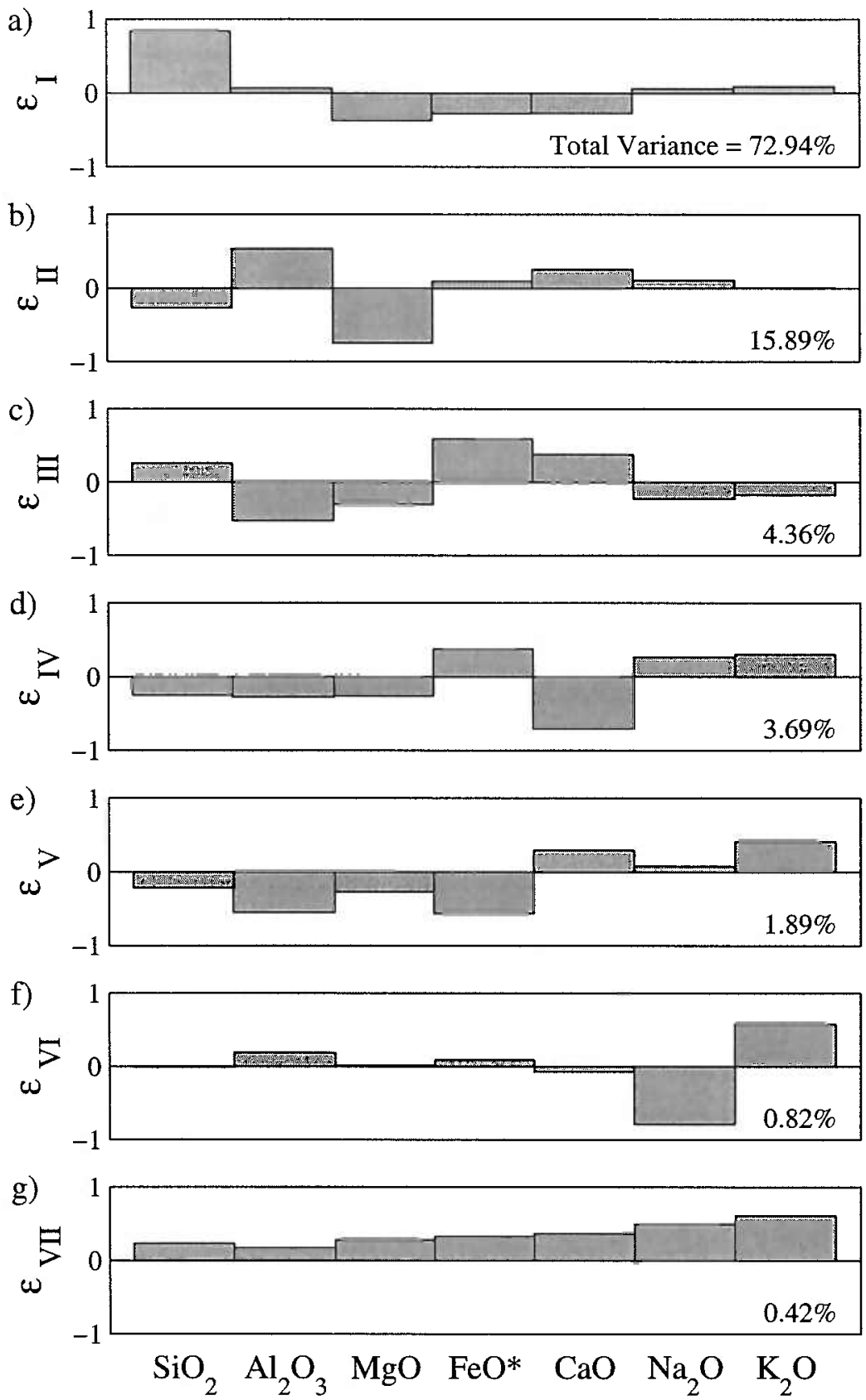

Figure 6-1: a-g) Bar plots of principal components given in Table 6.2. Percentage of total variance described by each component is shown. Linear combinations of components, $\epsilon_{\mathrm{I}}, \epsilon_{\mathrm{II}}, \epsilon_{\mathrm{III}}, \epsilon_{\mathrm{IV}}$, and $\epsilon_{\mathrm{V}}$ are used to construct the synthetic composition space from which crystallizing phase assemblages are calculated. 
Table 6.2: Eigenvectors and eigenvalues of the variance-covariance matrix in Table 6.1.

\begin{tabular}{lccccccc} 
& \multicolumn{7}{c}{ Eigenvector } \\
\cline { 2 - 8 } Oxide & $\epsilon_{\mathrm{I}}$ & $\epsilon_{\mathrm{II}}$ & $\epsilon_{\mathrm{III}}$ & $\epsilon_{\mathrm{IV}}$ & $\epsilon_{\mathrm{V}}$ & $\epsilon_{\mathrm{VI}}$ & $\epsilon_{\mathrm{VII}}$ \\
\hline $\mathrm{SiO}_{2}$ & 0.836 & -0.271 & 0.259 & -0.250 & -0.216 & -0.008 & 0.227 \\
$\mathrm{Al}_{2} \mathrm{O}_{3}$ & 0.067 & 0.533 & -0.522 & -0.276 & -0.547 & 0.187 & 0.168 \\
$\mathrm{MgO}$ & -0.370 & -0.748 & -0.299 & -0.259 & -0.270 & 0.013 & 0.273 \\
$\mathrm{FeO}$ & -0.274 & 0.093 & 0.590 & 0.372 & -0.564 & 0.088 & 0.322 \\
$\mathrm{CaO}$ & -0.269 & 0.252 & 0.377 & -0.706 & 0.295 & -0.073 & 0.362 \\
$\mathrm{Na}_{2} \mathrm{O}$ & 0.062 & 0.107 & -0.226 & 0.260 & 0.079 & -0.786 & 0.491 \\
$\mathrm{~K}_{2} \mathrm{O}$ & 0.090 & 0.004 & -0.172 & 0.299 & 0.412 & 0.577 & 0.608 \\
\hline Eigenvalues & 113.42 & 24.71 & 6.78 & 5.74 & 2.93 & 1.27 & 0.65 \\
\% Variance & 72.94 & 15.89 & 4.36 & 3.69 & 1.89 & 0.82 & 0.42 \\
\hline
\end{tabular}

are illustrated as bar diagrams in Figure 6-1. Note that eigenvectors $\epsilon_{\mathrm{I}}$ and $\epsilon_{\mathrm{II}}$ explain $\sim 89 \%$ of the total variance in the oxide space, as compared to only $\sim 73 \%$ by $\mathrm{SiO}_{2}$ and $\mathrm{MgO}$.

The applicability of the principal components to natural igneous systems can be interpreted in terms of the relationships between the major element oxides in each eigenvector. For example, the anti-correlation of $\mathrm{SiO}_{2}$ to $\mathrm{MgO}, \mathrm{FeO}^{*}$, and $\mathrm{CaO}$ in $\epsilon_{\mathrm{I}}$ nicely describes the transition from felsic to mafic compositions, which accounts for $~ 73 \%$ of the variance in the IGBA composition space. Likewise, the strong anti-correlation between $\mathrm{Al}_{2} \mathrm{O}_{3}$ and $\mathrm{MgO}$ in $\epsilon_{\mathrm{II}}$ represents the progression from lavas to cumulates, and the anti-correlation of $\mathrm{MgO}$ and $\mathrm{FeO}$ in $\epsilon_{\mathrm{IV}}$ separates primitive from relatively evolved, Fe-rich, basaltic lavas and cumulate gabbros.

\subsubsection{Generation of Synthetic Bulk Compositions}

Using the principal components calculated above, we now construct a set of synthetic bulk compositions that spans the full range of igneous composition space. Synthetic compositions are calculated by taking linear combinations of $\epsilon_{\mathrm{I}}, \epsilon_{\mathrm{II}}, \epsilon_{\mathrm{III}}, \epsilon_{\mathrm{IV}}$, and $\epsilon_{\mathrm{V}}$. We chose to sample only the first five principal components because these account for $\sim 99 \%$ of the total variance of the system. To determine the range over which to sample each component, we construct histograms of the principal component scores of the original oxide data 
a)

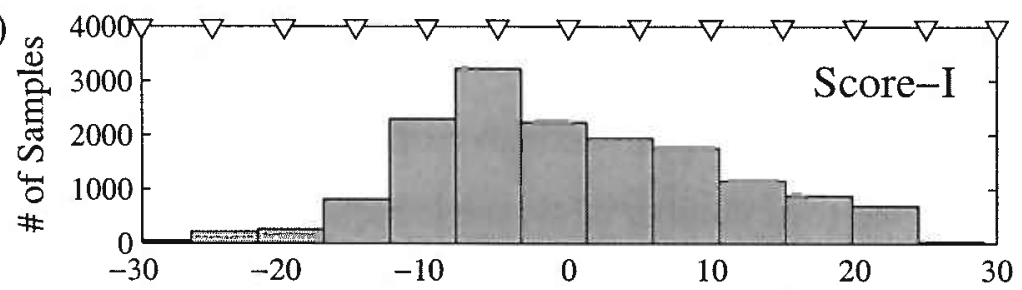

b)

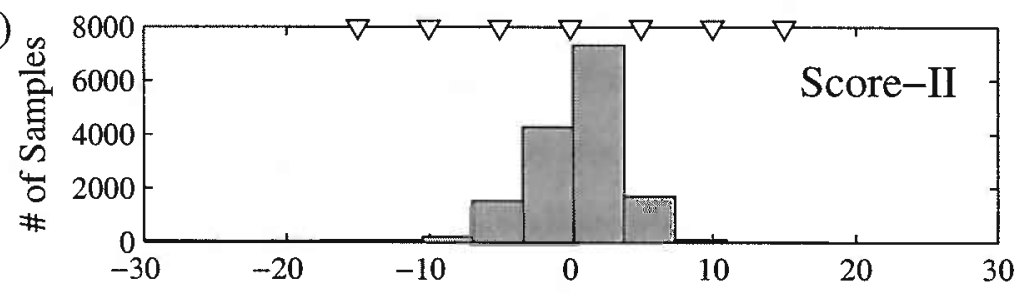

c)

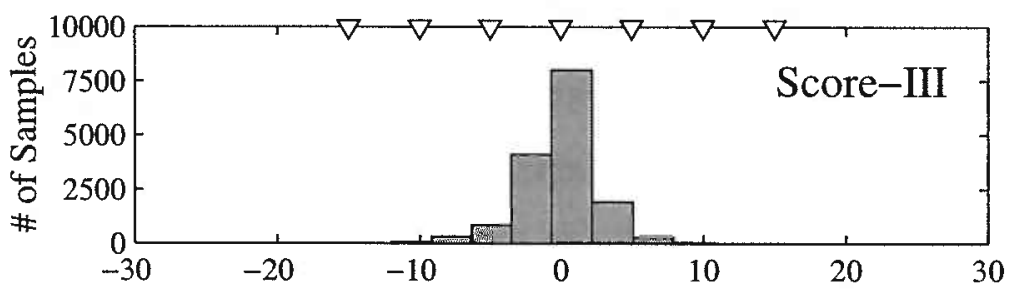

d)

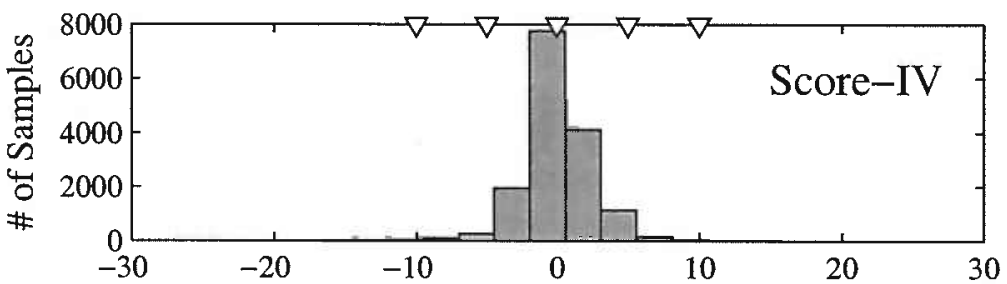

e)

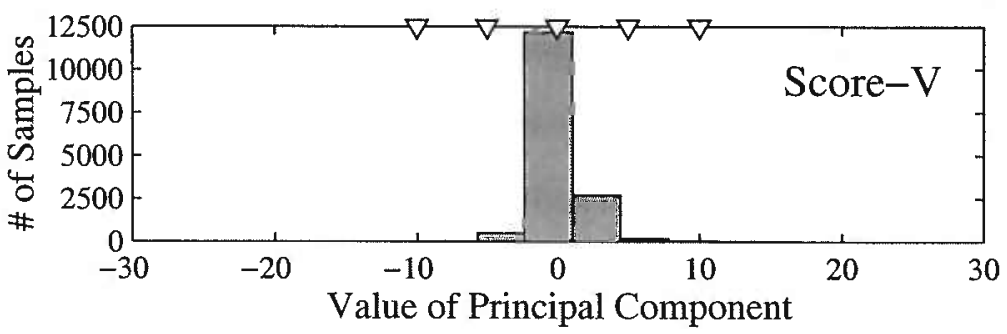

Figure 6-2: Histograms of principal component scores for a) $\left.\left.\epsilon_{\mathrm{I}}, \mathrm{b}\right) \epsilon_{\mathrm{II}}, \mathrm{c}\right) \epsilon_{\mathrm{III}}$, d) $\epsilon_{\mathrm{IV}}$, and e) $\epsilon_{\mathrm{V}}$. Triangles represent sampling points used to generate synthetic compositions. Principal component scores are referenced to the mean composition of the IGBA oxide data: 54.92 wt $\% \mathrm{SiO}_{2}, 15.66 \mathrm{wt} \% \mathrm{Al}_{2} \mathrm{O}_{3}, 5.16$ wt $\% \mathrm{MgO}, 7.83$ wt\% $\mathrm{FeO}^{*}, 7.09$ wt $\% \mathrm{CaO}, 3.47$ wt $\%$ $\mathrm{Na}_{2} \mathrm{O}$, and $2.01 \mathrm{wt} \% \mathrm{~K}_{2} \mathrm{O}$. 
(Figure 6-2). The principal component scores are calculated by projecting the original data onto each of the five eigenvectors. A uniform sampling scheme (see Figure 6-2) is chosen to insure that sampling biases in the IGBA database do not cause us to under-represent certain regions of igneous composition space. All linear combinations that result in either a negative value for one of the major element oxides or a total weight percent $<95 \%$ are eliminated, reducing the total number of synthetic bulk compositions from 15,925 to 479 .

Figure 6-3 shows a comparison of the major element oxide distributions from the IGBA database and the synthetic compositions. The linear sampling scheme results in oxide distributions for the synthetic data that are broader than those for the original IGBA data. Our goal, however, is not to create a dataset that perfectly reproduces the major element chemistry of all igneous rocks, but rather to generate a set of bulk compositions that fully encompasses all possible igneous compositions. The synthetic distributions shown in Figure 6-3, therefore, satisfy our requirement, as the naturally occurring range of each major oxide is represented by the synthetic compositions.

\subsection{Calculation of $V_{p}$ for Igneous Composition Space}

To estimate seismic velocities for the bulk compositions in our synthetic data space, we calculate equilibrium mineral assemblages at various equilibrium conditions and use the elastic moduli of the stable phases to place bounds on the seismic velocities. A number of previous workers have used a norm-based approach to calculate hypothetical solid velocities for primary and residual liquids of mantle melts [e.g., White and McKenzie, 1989; Korenaga et al., 2002]. However, using CIPW norms to estimate equilibrium mineral assemblages rather than performing thermodynamic calculations, ignores the possible presence

of high pressure minerals such as garnet. Because of its high seismic velocity $\left(V_{p}>8.5\right.$ $\mathrm{km} / \mathrm{s}$ ), even small amounts of garnet may significantly influence the overall velocity of a mineral assemblage. Thus, to assess the full range of igneous compositions that are consistent with a given seismic velocity, it is necessary to use a thermodynamic approach when estimating the equilibrium mineral assemblages [e.g., Sobolev and Babeyko, 1994]. 

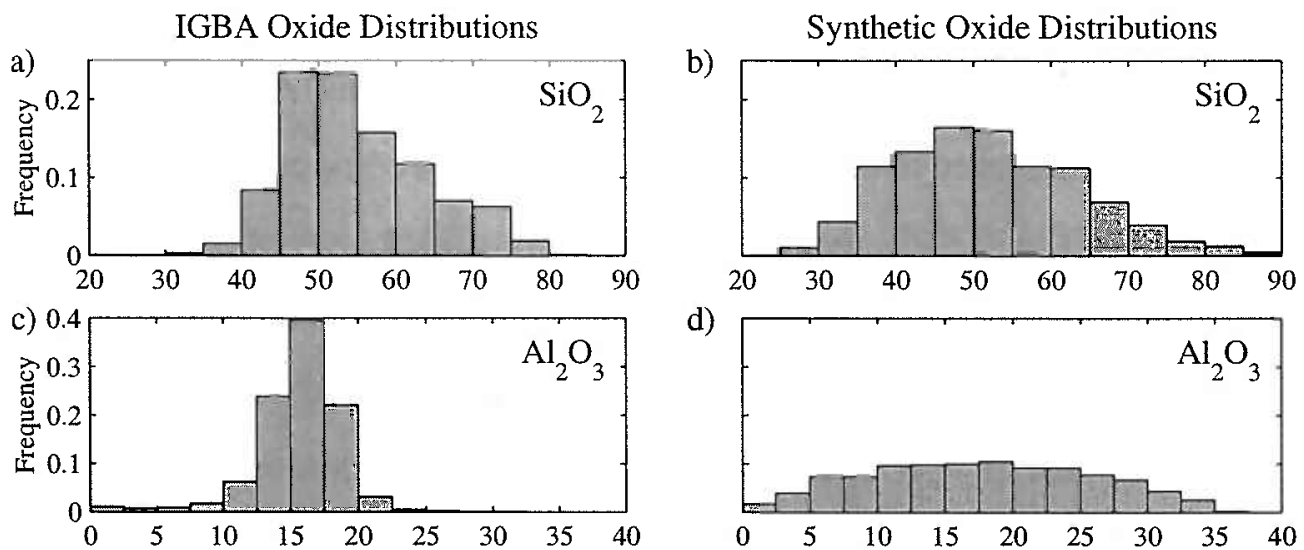

d)
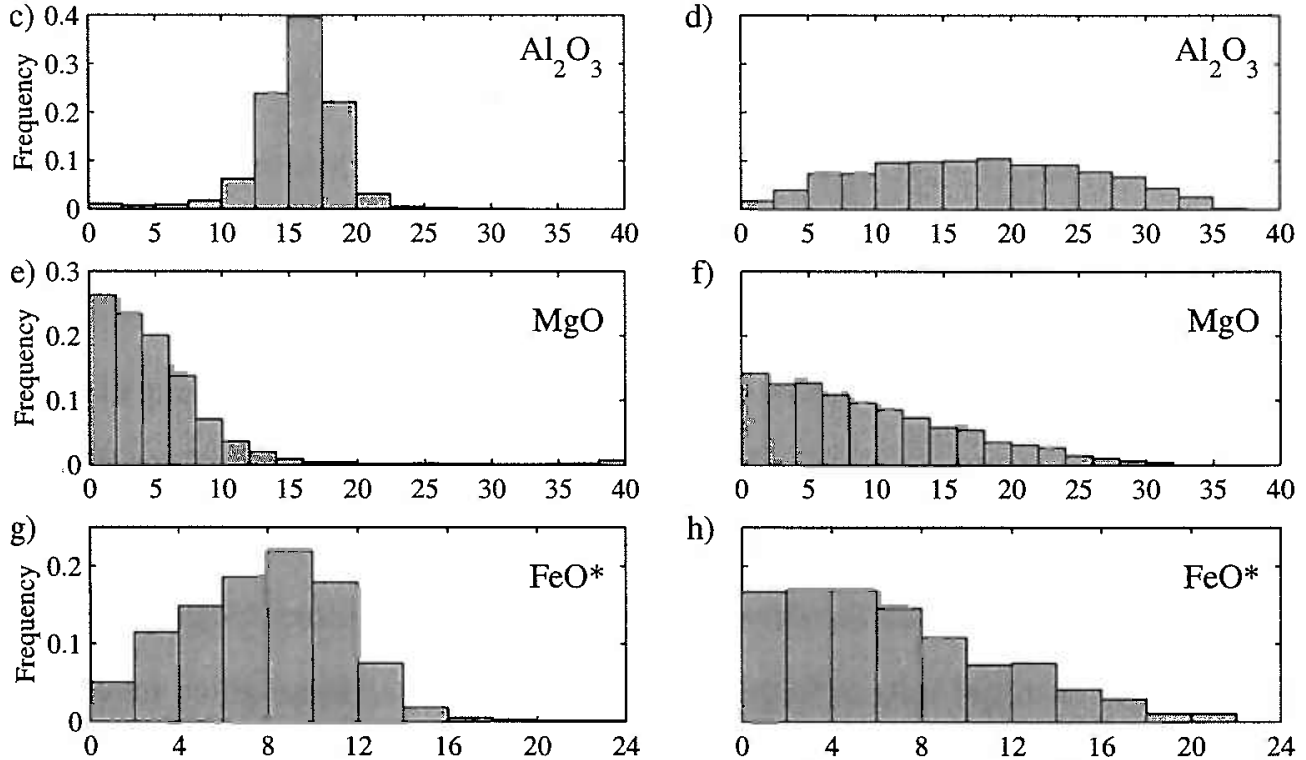

h)
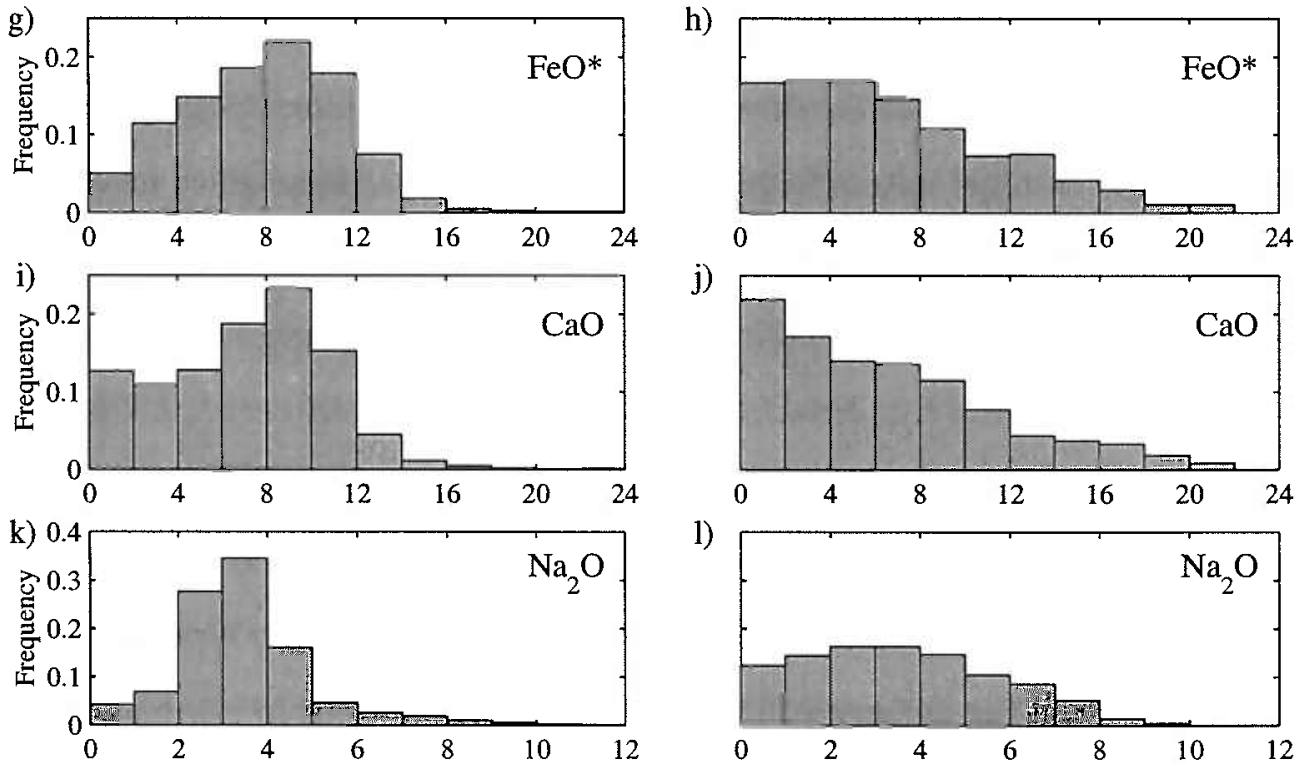

1)
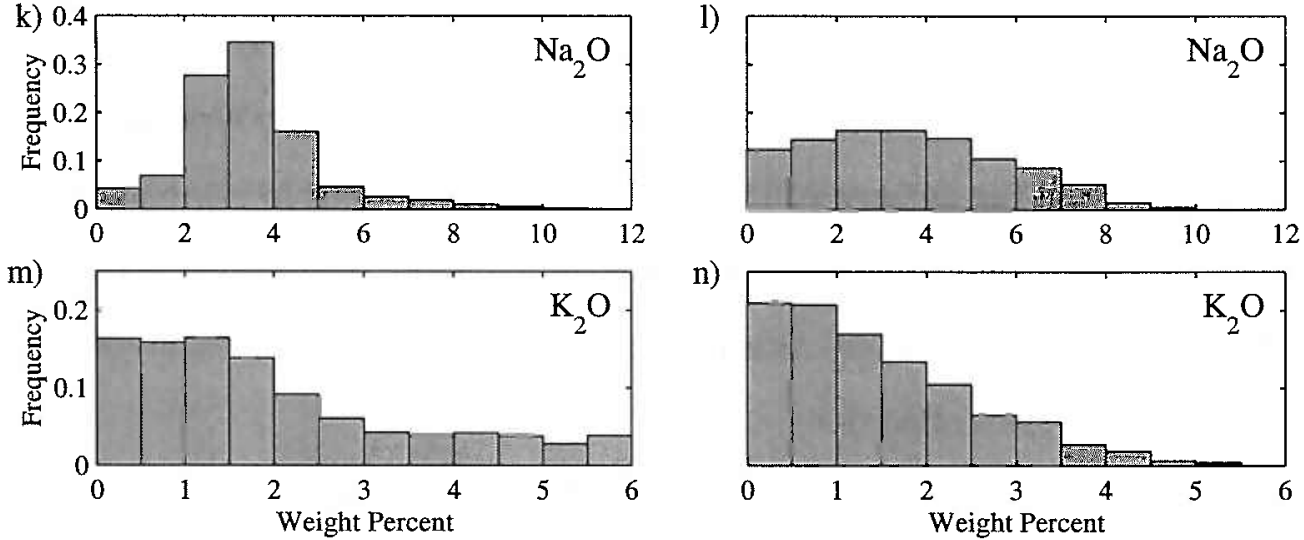

n)

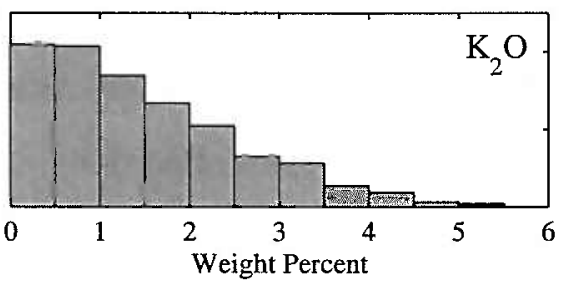

Figure 6-3: Observed frequency distributions for major oxides in IGBA database, a) $\mathrm{SiO}_{2}$, c) $\mathrm{Al}_{2} \mathrm{O}_{3}$, e) $\mathrm{MgO}$, g) $\mathrm{FeO}^{*}$, i) $\mathrm{CaO}$, k) $\mathrm{Na}_{2} \mathrm{O}$, m) $\mathrm{K}_{2} \mathrm{O}$, and calculated frequency distributions for synthetic compositions, b) $\mathrm{SiO}_{2}$, d) $\mathrm{Al}_{2} \mathrm{O}_{3}$, f) $\mathrm{MgO}$, h) $\mathrm{FeO}^{*}$, j) $\mathrm{CaO}$, 1) $\mathrm{Na}_{2} \mathrm{O}$, n) $\mathrm{K}_{2} \mathrm{O}$. Note that synthetic oxide distributions are much broader than IGBA distributions. 
Subsolidus phase equilibrium calculations are performed using a program developed by Connolly [1990] and the thermodynamic database of Holland and Powell [1998]. Only the 7 major element oxides used to construct the synthetic bulk compositions were included in the calculations. $\mathrm{TiO}_{2}$ and $\mathrm{P}_{2} \mathrm{O}_{5}$ were excluded for simplicity, as they are unlikely to affect the calculated subsolidus modes. For each synthetic composition, crystallizing mineral assemblages were calculated for an equilibration temperature, $T_{e q}$, of $800^{\circ} \mathrm{C}$ and equilibration pressures, $P_{e q}$, of $0.5,1,2,4,6,8,10,12,15$, and 20 kbar. We choose a single equilibration temperature of $800^{\circ} \mathrm{C}$ because this is a likely, lower bound for net-transfer reactions (nucleation and growth of new mineral phases) under anhydrous, lower crustal conditions [e.g., Austrheim, 1998; Hacker et al., 2000, B. Hacker, pers. comm. 2001]. Equilibrium solutions were not found at one or more of the $P_{e q}, T_{e q}$ conditions for 56 of the 479 synthetic compositions. In general, these compositions lie near the limits of the synthetic data space and are probably not representative of naturally occurring igneous rock compositions.

The calculated mineral assemblages are then used to estimate seismic velocities for each synthetic composition at the 10 equilibrium conditions. The weight proportions of the equilibrium phases are first converted into volume proportions using the mineral densities. Hashin-Shtrikman bounds [e.g., Hashin and Shtrikman, 1963; Watt et al., 1976; Berryman, 1995] are then calculated for $V_{p}$ and $V_{s}$ of each multi-phase assemblage using the compilation of densities and elastic moduli from Sobolev and Babeyko [1994] and Bass [1995]. For the bulk compositions in the synthetic data space the bounds are typically tighter than $0.12 \mathrm{~km} / \mathrm{s}$, and we use the average of the upper and lower bounds to estimate the velocity. Figure 6-4 illustrates a sample calculation for one of the compositions in the synthetic data space. We note that the velocities calculated in this manner assume an anhydrous mineral assemblage with no residual porosity.

Because pressure and temperature can strongly affect the seismic velocities of crustal and mantle rocks [e.g., Christensen, 1979], it is necessary to use laboratory derived pressure and temperature derivatives to correct $V_{p}$ and $V_{s}$ to a reference pressure, $P_{\text {ref }}$, and temperature, $T_{r e f}$. Note that $P_{r e f}$ and $T_{r e f}$ are independent of $P_{e q}$ and $T_{e q}$, with the former representing the pressure and temperature conditions at which the seismic velocity is mea- 

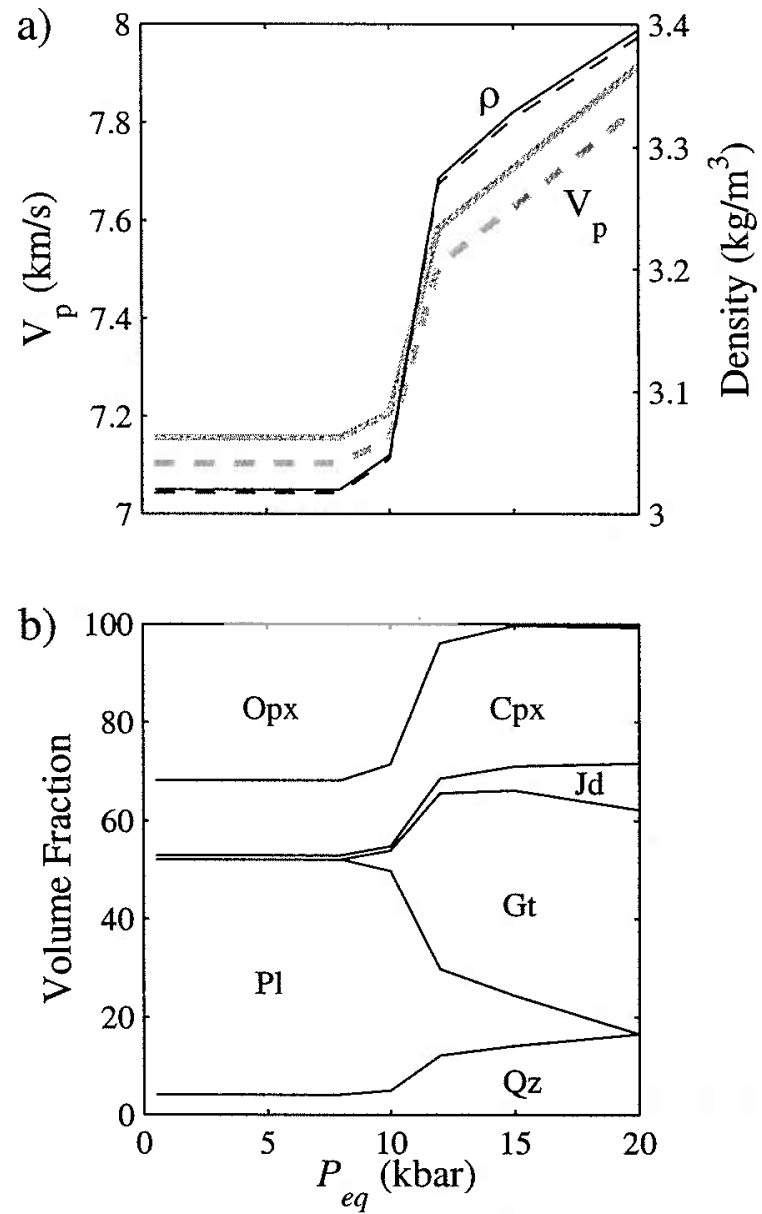

Figure 6-4: Sample calculation of the equilibrium mineral assemblage and corresponding seismic velocities for the synthetic bulk composition with $54.24 \mathrm{wt} \% \mathrm{SiO}_{2}, 14.27 \mathrm{wt} \%$ $\mathrm{Al}_{2} \mathrm{O}_{3}, 12.24$ wt $\% \mathrm{MgO}, 6.98 \mathrm{wt} \% \mathrm{FeO}^{*}, 10.88 \mathrm{wt} \% \mathrm{CaO}, 1.25 \mathrm{wt} \% \mathrm{Na}_{2} \mathrm{O}$, and $0.05 \mathrm{wt} \%$ $\mathrm{K}_{2} \mathrm{O}$. a) Calculated density (black) and $V_{p}$ (gray) for the crystallizing assemblage at 1) $P_{\text {ref }}=200^{\circ} \mathrm{C}$ and $T_{r e f}=3 \mathrm{kbar}$ (solid curves) and 2) $P_{\text {ref }}=600^{\circ} \mathrm{C}$ and $T_{r e f}=10 \mathrm{kbar}$ (dashed curves). b) Volume proportions of the crystallizing phases as a function of $P_{e q}$ $T_{e q}=800^{\circ} \mathrm{C}$ for all calculations. Mineral abbreviations are (Opx) orthopyroxene, $(\mathrm{Cpx})$ clinopyroxene, $(\mathrm{Pl})$ plagioclase, $(\mathrm{Gt})$ garnet, $(\mathrm{Qz})$ quartz, and $(\mathrm{Jd})$ jadite. 
sured and the latter referring to the conditions under which the sample equilibrated. We calculate values of $P_{\text {ref }}$ and $T_{\text {ref }}$ along a crustal geotherm for a depth range of 5-50 km, assuming [Pollack and Chapman, 1977]

$$
P_{r e f}=\rho g z
$$

and

$$
T_{r e f}=T_{\mathrm{o}}+\frac{\left(q_{s}-q_{m}\right) d}{K}\left(1-\exp \left(\frac{-z}{d}\right)\right)+\frac{q_{m}}{K} z
$$

where $\rho$ is the average crustal density, $g$ is the gravitational acceleration, $z$ is depth, $T_{\mathrm{o}}$ is the surface temperature, $q_{s}$ is the surface heat flow, $q_{m}$ is the mantle (or reduced) heat flow, $d$ is a characteristic depth scale, and $K$ is the thermal conductivity. We assume $\rho=2900 \mathrm{~kg} / \mathrm{m}^{3}$, $T_{\mathrm{o}}=10^{\circ} \mathrm{C}, d=10 \mathrm{~km}, K=3.35 \mathrm{~W} \mathrm{~m}^{-1}{ }^{\circ} \mathrm{K}^{-1}$, and $q_{m}$ to be $60 \%$ of $q_{s}$ for all calculations. Values of $q_{s}=35,56$, and $90 \mathrm{~mW} \mathrm{~m}^{-2}$ are used to simulate cold, normal, and warm crustal geotherms, respectively. Thus, for each synthetic bulk composition, $V_{p}$ is calculated at 100 different combinations of $P_{e q}, T_{e q}$ and $P_{r e f}, T_{r e f}$ along a particular geotherm.

\subsubsection{Comparison to Laboratory Data}

Before analyzing the relationship between bulk composition and seismic velocity using the synthetic data space, it is necessary to evaluate the ability of our approach to reproduce seismic velocities for known igneous compositions. We compile high-quality laboratory data on 139 igneous and meta-igneous rocks for which both $V_{p}$ and major element composition are measured [Manghnani et al., 1974; Kern and Richter, 1981; Kern and Schenk, 1985; Christensen and Szymanski, 1988; Kern and Schenk, 1988; Christensen, 1989; Burke

and Fountain, 1990; Fountain et al., 1990; Kern and Tubia, 1993; Miller and Christensen, 1994]. We limit our compilation to measurements of $V_{p}$ at $\geq 6 \mathrm{kbar}$ to simulate confining pressures appropriate for the lower crust. For samples in which velocities were measured in three orthogonal directions, the average value is used in the comparison. Equilibrium 
phase assemblages are calculated for the reported major element compositions over the range: $T_{e q}=500-1000^{\circ} \mathrm{C}$ and $P_{e q}=2-12 \mathrm{kbar} . V_{p}, V_{s}$, and density are calculated for each equilibrium mode using the Hashin-Shtrikman bounds and the best-fit $V_{p}$ for each composition is determined. We choose to fit only $V_{p}$ because most density measurements were made at ambient pressure and temperature, increasing the risk of residual porosity, and $V_{s}$ data were available for only a small subset of samples. However, both the densities and the $V_{s}$ values calculated from the mineral assemblages fit for $V_{p}$ compare well to the laboratory data (Figure 6-5). The values of $P_{e q}$ and $T_{e q}$ corresponding to the best-fit $V_{p}$ for each sample are illustrated in Figure 6-5b. Based on our approach, the data for samples analyzed in the laboratory appear to be characterized by two principal styles of equilibration. The first occurs at high pressure and high temperature, while the second is characterized by equilibration at low pressure, but over a wide range of temperatures.

We also compared the calculated equilibrium modes fit for $V_{p}$ with the observed modes for samples on which point-counts were performed (Figure 6-5e-j). Overall the calculated modes compare well with the observed modes. The most important discrepancy is found in the prediction of olivine, where our equilibrium calculations often predict up to $20 \mathrm{wt} \%$ olivine even though none is observed in the laboratory sample. The cause of this systematic overprediction is probably related to the fact that we ignore both $\mathrm{Fe}^{3+}$ and $\mathrm{Cr}$ in our equilibrium calculations. The absence of these components suppresses reactions in which olivine reacts to form either spinel or magnetite, and thus leaves an excess of olivine in the calculated mineral assemblages.

A comparison of the best-fit $V_{p}$ data to the laboratory measurements at confining pressures $\geq 6$ kbar shows the calculated velocities systematically overpredict the experimental data by $0.05 \pm 0.12 \mathrm{~km} / \mathrm{s}$ (Figure 6-5). This systematic overprediction may be caused by residual porosity or the presence of alteration phases in the laboratory samples [e.g., Jackson et al., 1990]. To isolate the effect of porosity, we compare the best-fit $V_{p}$ to laboratory data for confining pressures ranging from 0.1 to 10 kbars (Figure 6-6). In general, the residual between the best-fit and experimental velocities decreases with increasing confining pressure. At pressures $\leq 3 \mathrm{kbars}$, the calculated velocities overpredict the experimental 
Figure 6-5: (facing page) Comparison of calculated and laboratory determined $V_{p}$ in 139 igneous and meta-igneous rocks at $T_{r e f}=25^{\circ} \mathrm{C}$ and $P_{r e f} \geq 6 \mathrm{kbar}$ [Manghnani et al., 1974; Kern and Richter, 1981; Kern and Schenk, 1985; Christensen and Szymanski, 1988; Kern and Schenk, 1988; Christensen, 1989; Burke and Fountain, 1990; Fountain et al., 1990; Kern and Tubia, 1993; Miller and Christensen, 1994]. $V_{p}, V_{s}$, and density are calculated for each bulk composition from the equilibrium mode over a range of $T_{e q}=500-1000^{\circ} \mathrm{C}$ and $P_{e q}=2-12 \mathrm{kbar}$. The best-fit $V_{p}$ is then determined for each sample over the range of equilibrium conditions. a) Best-fit $V_{p}$ versus experimentally determined $V_{p}$. For experimental data in which velocities were measured in multiple directions the average value is used in the comparison. Gray symbols represent samples containing garnet, black symbols represent samples that are garnet-free. The residuals of the best-fit data are calculated relative to a one-to-one correlation (black line), and have a mean $=0.047$, median $=0.015$, and standard deviation $=0.125 \mathrm{~km} / \mathrm{s}$. b) 2-D histogram of best-fitting $T_{e q}$ and $P_{e q}$. Italic numbers indicate number of samples in each bin. c) Calculated versus laboratory measured density using the equilibrium modes fit for $V_{p}$ (mean $=0.038$, median $=0.032$, and standard deviation $\left.=0.080 \mathrm{~g} / \mathrm{cm}^{3}\right)$. d) Calculated versus laboratory $V_{s}$ using equilibrium modes fit for $V_{p}$ (mean $=0.037$, median $=0.036$, and standard deviation $=0.114 \mathrm{~km} / \mathrm{s}$, $\mathrm{N}=66$ ). e-j) Comparison of major minerals in calculated and observed modes for samples on which point-count analyses were performed $(\mathrm{N}=50)$.

data by $>0.15 \mathrm{~km} / \mathrm{s}$. However, for pressures $\geq 4 \mathrm{kbar}$ the best-fit and experimental velocities are found to agree within error. This implies that microcracks are not completely closed below pressures of $\sim 3 \mathrm{kbar}$ or corresponding crustal depths shallower than $\sim 10 \mathrm{~km}$. Similar conclusions have been drawn from experimental studies that observe an increase in $V_{p}$ with confining pressure from $0-2 \mathrm{kbar}$ that is significantly more rapid than that predicted from the mineral compressibilities alone [e.g., Kern and Richter, 1981; Kern and Schenk, $1985,1988]$

The scatter in the residuals is probably related to anisotropy in the laboratory data. Christensen and Mooney [1995] found the average $V_{p}$ anisotropy for igneous rocks to be $\sim 1-2 \%$ (or $0.07-0.14 \mathrm{~km} / \mathrm{s}$ for $V_{p}=6.8 \mathrm{~km} / \mathrm{s}$ ). Of the 105 samples in our compilation for which $V_{p}$ was measured in multiple orthogonal directions, the mean standard deviation within each sample was $\pm 0.16 \mathrm{~km} / \mathrm{s}$. Thus, our approach for estimating $V_{p}$ from bulk composition is accurate within the precision of the experimental data, and we conclude that $V_{p}$ calculated in this manner will be representative of the true seismic velocity of an equilibrium mineral assemblage in the absence of residual porosity. 

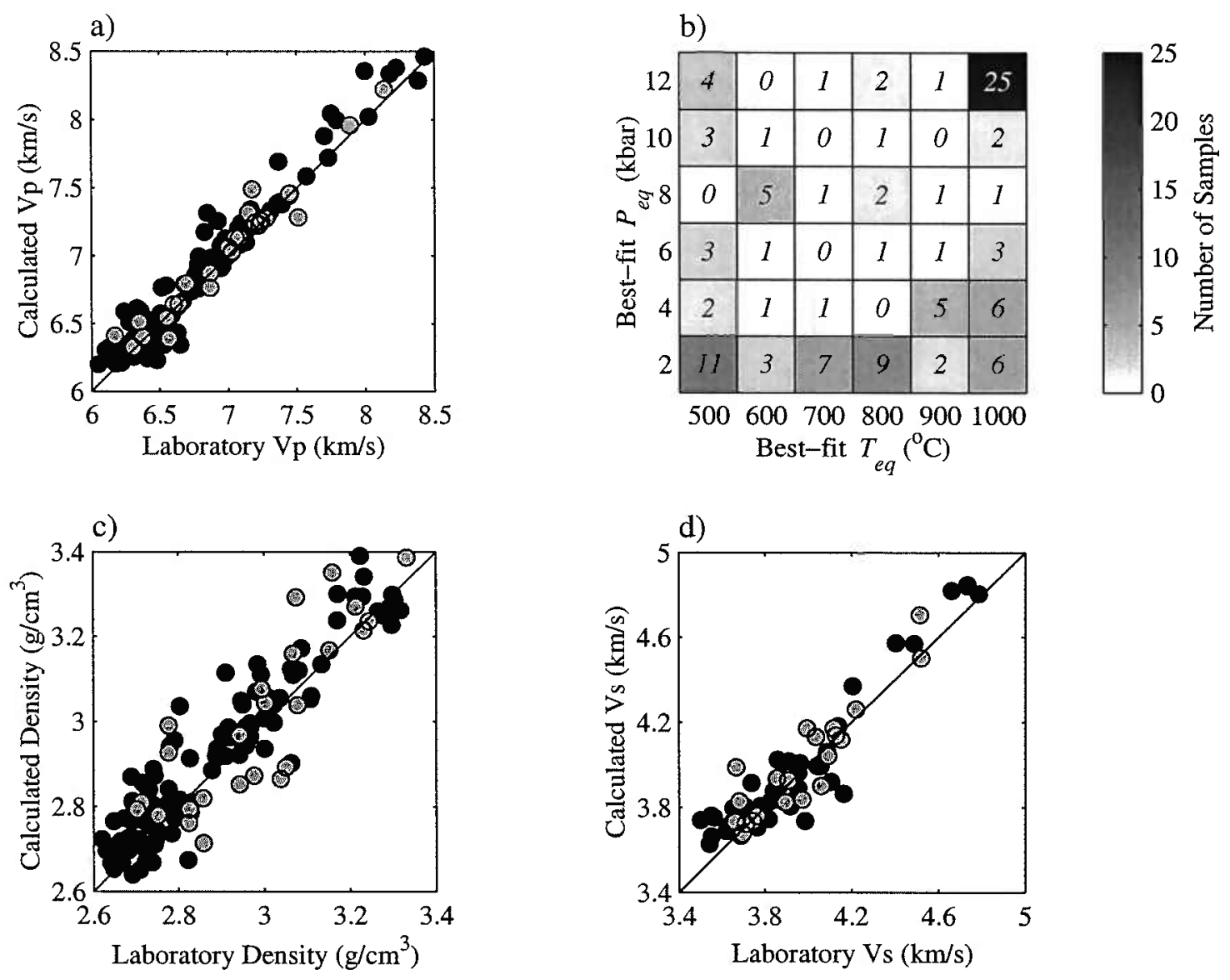

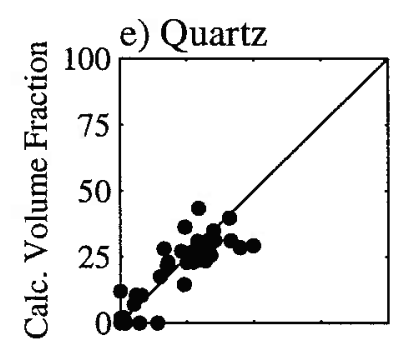

f) Plagioclase
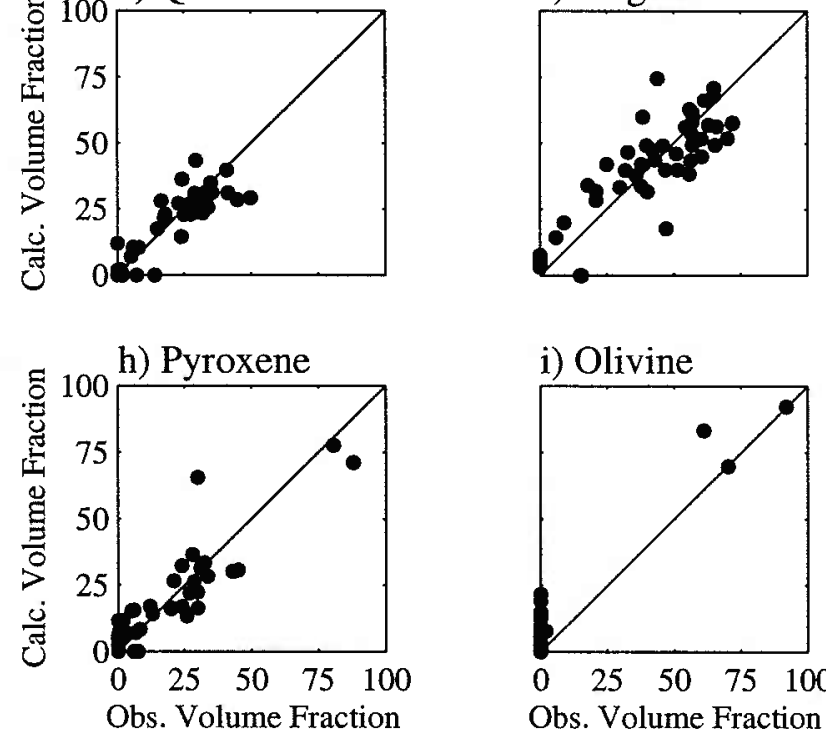

i) Olivine

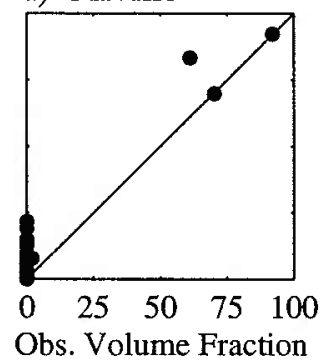

g) K-Spar

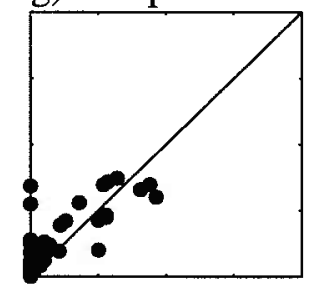

j) Garnet

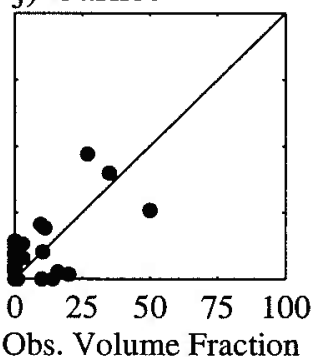




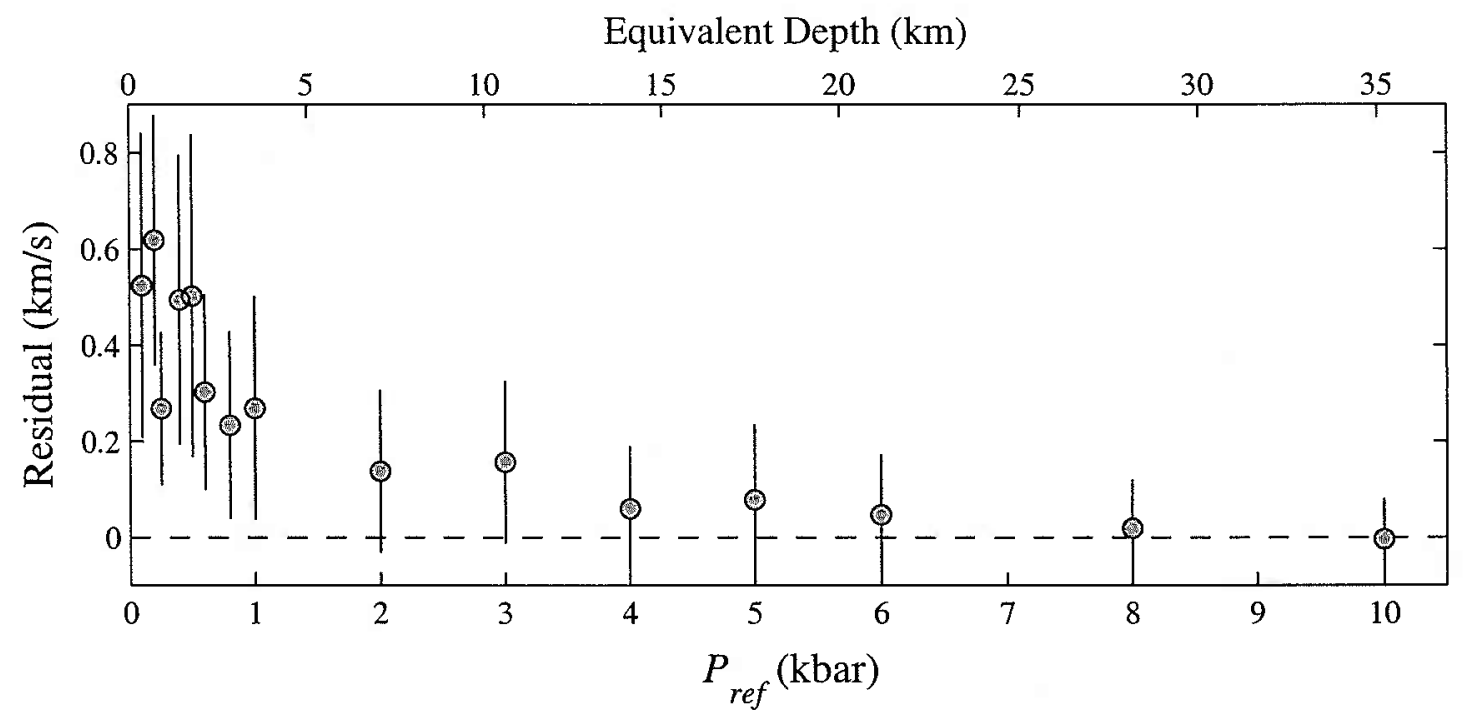

Figure 6-6: Comparison of the average residual between the best-fit $V_{p}$ and the laboratory data for confining pressures ranging from 0.1 to $10 \mathrm{kbars}$. Error bars represent the standard deviation in the residuals for each pressure. At confining pressures $\geq 4 \mathrm{kbars}$, the calculated and experimental velocities agree within error. However, for pressures $\leq 3 \mathrm{kbar}$, the best-fit $V_{p}$ overpredicts the experimental data by $>0.15 \mathrm{~km} / \mathrm{s}$.

\subsection{Relating $V_{p}$ to Igneous Rock Composition}

The seismic velocities calculated from the synthetic data are now used to develop a number of useful tools to infer compositional information from measurements of seismic velocity. Multiple linear regression is used to construct a direct relationship between $V_{p}$ and major element oxide composition over the full spectrum of igneous composition space. The resulting relationship can be used to predict $V_{p}$ from bulk composition and further to constrain mantle melting parameters (i.e., pressure and melt fraction) in conjunction with seismic observations [e.g., Kelemen and Holbrook, 1995; Korenaga et al., 2002]. Finally, by inverting directly for major element oxide chemistry as a function of $V_{p}$, we address the fundamental problem facing all seismic inferences of crustal composition; namely if only $V_{p}$ is known, what is full range of compositions that are consistent with the seismic observations? 


\subsubsection{Direct Relationship between $V_{p}$ and Major Element Oxide Com- position}

Kelemen and Holbrook [1995] used experimental data to derive an empirical relationship between $V_{p}$ and major element oxide composition for garnet-free igneous and meta-igneous rocks at a reference state of $600 \mathrm{MPa}$ and $400^{\circ} \mathrm{C}$,

$$
V_{p}=7.854-0.024 \mathrm{wt} \% \mathrm{SiO}_{2}+0.029 \mathrm{wt} \% \mathrm{MgO}
$$

This function has an accuracy of $\pm 0.19 \mathrm{~km} / \mathrm{s}(1 \sigma)$ and was not improved by adding additional oxides to the regression. A significant source of error in Equation 6.3 is the difficulty in making seismic velocity measurements on laboratory samples discussed above. Further, the applicability of this relationship is limited by the range of compositions for which experimental data were available.

Following a similar approach to Kelemen and Holbrook [1995], we use the seismic velocities calculated from the synthetic data to construct a linear relationship between $V_{p}$ and composition that is valid for all igneous compositions. Multiple linear regression results in the following function relating $V_{p}$ to $\mathrm{SiO}_{2}, \mathrm{MgO}$, and $\mathrm{CaO}$ for all $P_{r e f}$ and $T_{r e f}$ conditions along a normal crustal geotherm in the depth range from 5-50 km (Figure 6-7),

$$
V_{p}=6.841-0.010 \mathrm{wt} \% \mathrm{SiO}_{2}+0.039 \mathrm{wt} \% \mathrm{MgO}+0.041 \mathrm{wt} \% \mathrm{CaO}
$$

This function has an accuracy of $\pm 0.12 \mathrm{~km} / \mathrm{s}(1 \sigma)$. We choose to include only $\mathrm{SiO}_{2}, \mathrm{MgO}$, and $\mathrm{CaO}$ in the analysis as the addition of other oxides does not significantly improve the fit, and regression with only $\mathrm{SiO}_{2}$ and $\mathrm{MgO}$ is insufficient to explain the variation in the $V_{p}$ data (Table 6.3). Equilibrium modes with $>25 \mathrm{wt} \%$ garnet were eliminated from the regression because garnet in these abundances is unlikely in crustal rocks and substantially degrades the quality of the fit in Equation 6.4 (with garnet, $1 \sigma= \pm 0.34 \mathrm{~km} / \mathrm{s}$ ). Specifically, $\sim 70 \%$ of all modes with $>25 \mathrm{wt} \%$ garnet equilibrated at $P_{e q} \geq 15 \mathrm{kbars}$, and thus these high garnet modes are only likely to be stable in regions where crustal thickness exceeds $50 \mathrm{~km}$. 

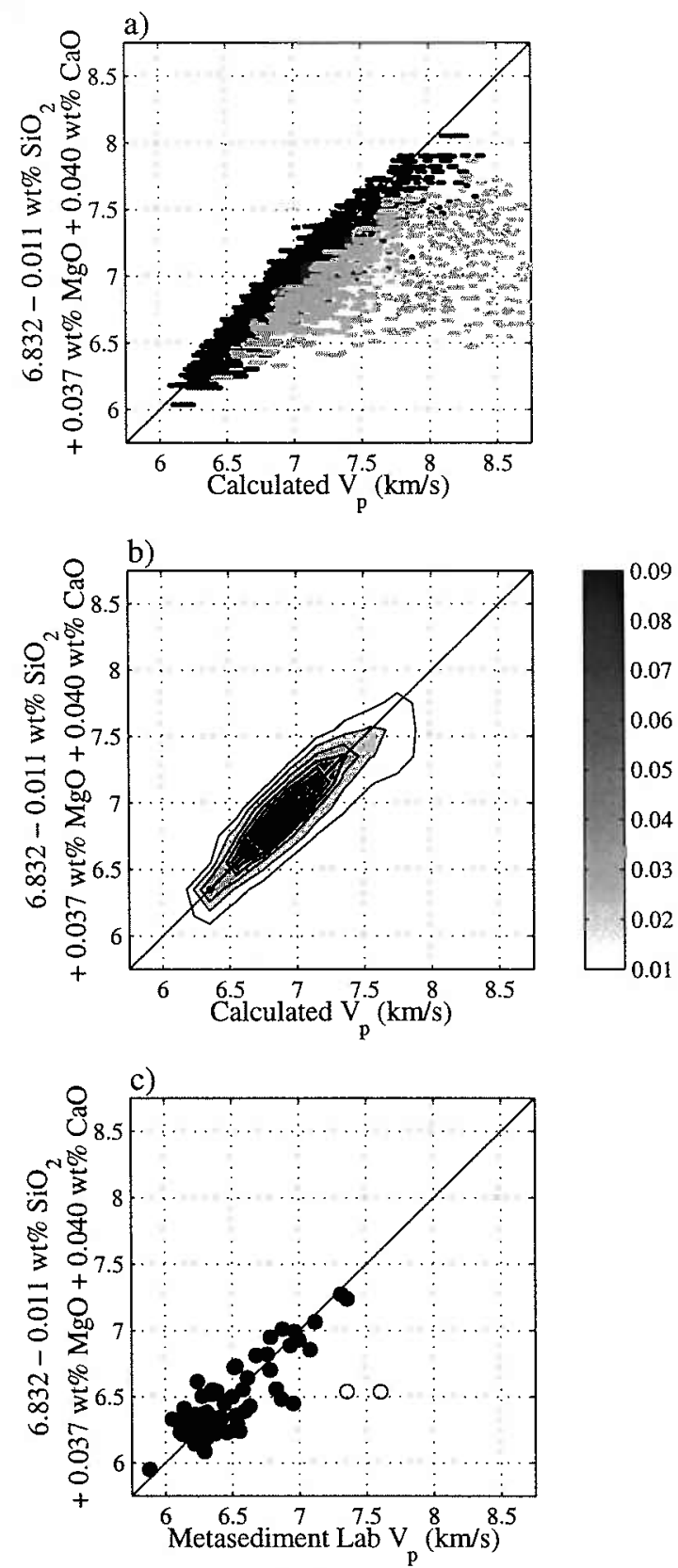

Figure 6-7: a) Results of multiple linear regression fit of major element oxide composition to $V_{p}$ calculated from the synthetic data along a normal crustal geotherm. Gray symbols represent synthetic compositions containing $>25 \mathrm{wt} \%$ garnet $(\mathrm{N}=8,140)$, which were not included in the regression. The resulting function is characterized by $N=30,520, \mathrm{r}^{2}=0.91$, and $1 \sigma= \pm 0.12$ $\mathrm{km} / \mathrm{s}$. The addition of other oxides, such as $\mathrm{Al}_{2} \mathrm{O}_{3}, \mathrm{FeO}, \mathrm{Na}_{2} \mathrm{O}$, and $\mathrm{K}_{2} \mathrm{O}$ did not significantly improve the fit (see Table 6.3). b) Data density plot, calculated by contouring the normalized number of observations in $0.1 \times 0.1 \mathrm{~km} / \mathrm{s}$ bins. Note that the garnet bearing samples do not represent a signficant fraction of the data space. c) Comparison of laboratory data with $V_{p}$ calculated from Equation 6.4 for 69 metasedimentary rocks. After eliminating two outliers (open symbols) the residuals have a mean $=0.03 \mathrm{~km} / \mathrm{s}$ and a stardard deviation $=0.17 \mathrm{~km} / \mathrm{s}$. 
Table 6.3: Results of multiple linear regression to relate $V_{p}$ to major element oxide composition for the synthetic data space. Results are shown for $V_{p}$ calculated from the synthetic bulk compositions at all $P_{r e f}$ and $T_{r e f}$ conditions in the depth range from 5-50 km along a cold, normal, and warm crustal geotherm. KH95 illustrates the empirical relation derived by Kelemen and Holbrook [1995] from laboratory data at a reference state of $600 \mathrm{MPa}$ and $400^{\circ} \mathrm{C}$.

\begin{tabular}{lcccccc} 
& \multicolumn{7}{c}{ Regression Coefficients } \\
\cline { 2 - 7 } Oxide & \multicolumn{7}{c}{$q_{s}=56 \mathrm{~mW} \mathrm{~m}^{-2}$} & \multicolumn{3}{c}{$35 \mathrm{~m} \mathrm{~m}^{-2}$} & $90 \mathrm{~mW} \mathrm{~m}^{-2}$ & $\mathrm{KH} 95$ \\
\hline $\mathrm{SiO}_{2}$ & -0.010 & -0.842 & -0.014 & -0.009 & -0.011 & -0.024 \\
$\mathrm{Al}_{2} \mathrm{O}_{3}$ & - & -0.714 & - & - & - & - \\
$\mathrm{MgO}$ & 0.039 & -0.784 & 0.034 & 0.040 & 0.037 & 0.029 \\
$\mathrm{FeO} *$ & - & -0.778 & - & - & - & - \\
$\mathrm{CaO}$ & 0.041 & -0.837 & - & 0.041 & 0.040 & - \\
$\mathrm{Na}_{2} \mathrm{O}$ & - & -1.309 & - & - & - & - \\
$\mathrm{K}_{2} \mathrm{O}$ & - & -0.527 & - & - & - & - \\
$\mathrm{Constant}$ & 6.841 & 89.037 & 7.368 & 6.846 & 6.832 & 7.854 \\
\hline $1 \sigma$ & 0.122 & 0.117 & 0.224 & 0.128 & 0.120 & 0.19 \\
$\mathrm{r}^{2}$ & 0.914 & 0.921 & 0.708 & 0.906 & 0.915 & 0.89 \\
\hline
\end{tabular}

We also tested the sensitivity of the regression coefficients to the crustal geotherm by recalculating the regression using $V_{p}$ data corrected for $P_{r e f}$ and $T_{r e f}$ along both a cold and warm geotherm (Table 6.3). Calculating $V_{p}$ for each synthetic bulk composition using the regression functions derived for the cold and warm geotherms results in average velocity differences of 0.08 and $-0.08 \mathrm{~km} / \mathrm{s}$, respectively, from the values calculated using Equation 6.4. These differences are consistent with the predicted variations in $V_{p}$ calculated along each geotherm using typical pressure $\left(\sim 2 \times 10^{-4} \mathrm{~km} \mathrm{~s}^{-1} \mathrm{MPa}^{-1}\right)$ and temperature derivatives $\left(\sim 4 \times 10^{-4} \mathrm{~km} \mathrm{~s}^{-1}{ }^{\circ} \mathrm{C}^{-1}\right)$ from the literature [e.g., Christensen, 1979; Kern, 1982; Fountain and Christensen, 1989; Jackson, 1991; Christensen and Mooney, 1995].

The relationship in Equation 6.4 was derived from the suite of synthetic compositions generated to span igneous composition space. However, while oceanic crust is igneous in origin, high-grade metamorphic assemblages may constitute a major component of the lower continental crust [e.g., Rudnick and Fountain, 1995]. Therefore, to insure that the derived relationship is not specific to igneous compositions it is desirable to test the ability of Equation 6.4 to reproduce seismic velocities for metamorphic rocks. Compiling labo- 
ratory data on 69 metasedimentary samples for which both composition and velocity data are available [Kern and Richter, 1981; Christensen and Szymanski, 1988; Kern and Schenk, 1988; Christensen, 1989; Burke and Fountain, 1990; Fountain et al., 1990; Kern and Tubia, 1993; Miller and Christensen, 1994], we use Equation 6.4 to predict $V_{p}$ from the reported major element oxides and compare the predicted values to laboratory measurements at $\geq 6$ kbar (Figure 6-7c). The resulting residuals were found to have a mean value of $0.03 \mathrm{~km} / \mathrm{s}$ and a stardard deviation of $0.17 \mathrm{~km} / \mathrm{s}$. We note that the standard deviation is slightly higher than the $1 \sigma$ error bounds for the regression in Equation 6.4. However, this discrepancy can be attributed to the high average anisotropy in metasediments, for which the mean standard deviation of orthogonal velocity measurements on a single sample is $\pm 0.28 \mathrm{~km} / \mathrm{s}$. Thus, it appears that Equation 6.4 can be used to predict $V_{p}$ from bulk composition for both igneous and metamorphic rocks.

\subsubsection{Relating $V_{p}$ to the Pressure and Fraction of Melting}

Using the relationship derived in Equation 6.4 we now investigate the connection between mantle melting systematics and the velocity structure of the resultant igneous crust [e.g., Kelemen and Holbrook, 1995; Korenaga et al., 2002]. Compiling high-quality data from anhydrous melting experiments of mantle peridotites [Kinzler and Grove, 1992; Kinlzer and Grove, 1993; Hirose and Kushiro, 1993; Baker and Stolper, 1994; Baker et al., 1995; Kinzler, 1997; Walter, 1998], we follow the approach of Kelemen and Holbrook [1995] to relate $V_{p}$ to the pressure and fraction of melting. $V_{p}$ is calculated for the experimental liquids using Equation 6.4, and multiple linear regression results in the following relationship valid for all $P_{\text {ref }}$ and $T_{r e f}$ conditions along a normal crustal geotherm in the depth range from 5-50 km (Figure 6-8),

$$
V_{p}=7.02+0.12 \mathrm{P}+1.04 \mathrm{~F}-0.002 \mathrm{P}^{2}-0.18 \mathrm{PF}+0.40 \mathrm{~F}^{2}
$$

where $\mathrm{P}$ is the pressure of melting in GPa and $\mathrm{F}$ is the melt fraction. The regression has a standard deviation of $\sim 0.04 \mathrm{~km} / \mathrm{s}$. However the true error associated with Equation 6.5 is 

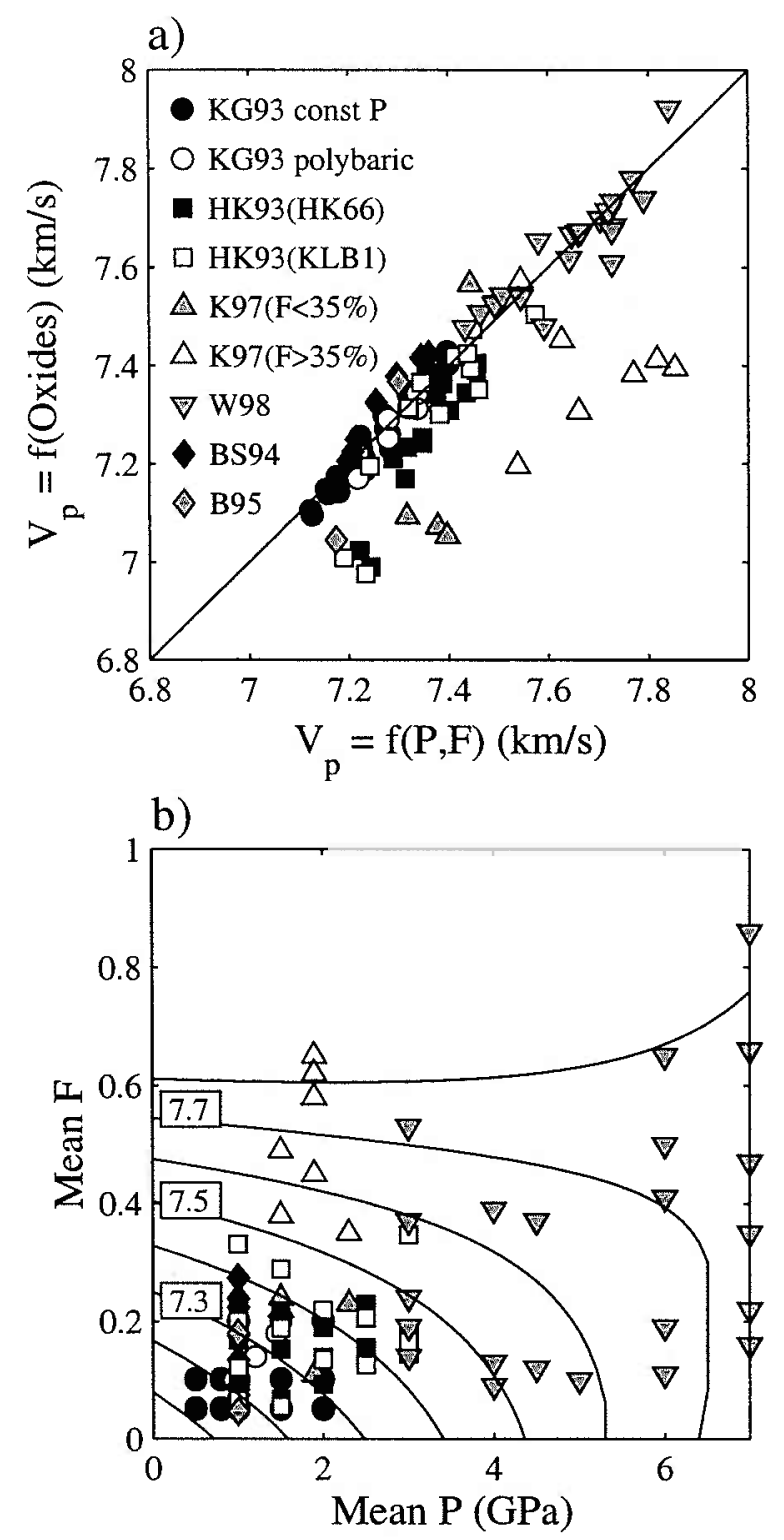

Figure 6-8: a) Relationship of $V_{p}$, calculated from Equation 6.4, to the pressure and fraction of melting for experimental and calculated anhydrous liquids in equilibrium with mantle lherzolite [Kinzler and Grove, 1992; Kinlzer and Grove, 1993; Hirose and Kushiro, 1993; Baker and Stolper, 1994; Baker et al., 1995; Kinzler, 1997; Walter, 1998]. Melting data of Baker and Stolper [1994] and Baker et al. [1995] were corrected after Hirschmann et al. [1998]. The resulting regression function is given in Equation 6.5 and is characterized by $\mathrm{N}=64, \mathrm{r}^{2}=0.96$, and $1 \sigma= \pm 0.04 \mathrm{~km} / \mathrm{s}$. Note that the mantle melts of Hirose and Kushiro [1993] and Kinzler [1997] were not included in the regression (see text for discussion). b) Mantle melt data as a function of the pressure and fraction of melting. Contours illustrate predicted seismic velocities calculated from Equation 6.5. 
higher, due to the uncertainty in estimating $V_{p}$ from the composition of the mantle melts. The data of Hirose and Kushiro [1993] and Kinzler [1997] were not included in the regression. Hirose and Kushiro [1993] calculated melt fraction from the $\mathrm{Na}_{2} \mathrm{O}$ concentration in the melt, which has been shown to overestimate the true melt fraction [e.g., Kogiso et al., 1998]. Further, half of the Hirose and Kushiro [1993] melting data are on pyroxenite, which may not represent a typical mantle source composition. The Kinzler [1997] data were not included due to the high melt fractions produced at relatively low average pressures $(F>35 \%$ in 7 of 11 experiments where $\mathrm{P} \leq 2.3 \mathrm{GPa})$. We note that using likely values of mean $\mathrm{P}$ and $\mathrm{F}$ for melting beneath mid-ocean ridges (i.e., $\overline{\mathrm{P}} \sim 1-2 \mathrm{GPa}$ and $\overline{\mathrm{F}} \sim 7-10 \%$ ) in Equation 6.5 gives $\mathrm{V}_{\mathrm{p}}>7.2 \mathrm{~km} / \mathrm{s}$, whereas seismic measurements of $V_{p}$ in average lower oceanic crust typically range from 6.9 to $7.0 \mathrm{~km} / \mathrm{s}$ [Raitt, 1963; Shor et al., 1970; Christensen and Salisbury, 1975; White et al., 1992]. We will discuss this discrepancy in more detail in Section 6.5.2.

\subsubsection{Inverting Bulk Composition from Measurements of $V_{p}$}

Previous workers have constrained the range of bulk compositions that are consisent with a particular seismic velocity by extrapolating laboratory measurements on a wide range of rock types to typical geologic conditions [e.g., Birch, 1958; Pakiser and Robinson, 1966; Fountain and Christensen, 1989; Holbrook et al., 1992; Christensen and Mooney, 1995; Rudnick and Fountain, 1995]. However, this approach does not predict formal bounds on bulk composition and is limited by the range of rock types for which laboratory data are available. In contrast, we use the seismic velocities calculated from the synthetic data to directly assess the range of igneous compositions that are consisent with a particular seismic velocity, assuming only a reference geotherm.

For seismic velocities ranging from $6.2-7.6 \mathrm{~km} / \mathrm{s}$, we search the synthetic data space for all bulk compositions that result in a $V_{p}$ within $\pm 0.1 \mathrm{~km} / \mathrm{s}$ of the desired velocity at $P_{\text {ref }}$ and $T_{\text {ref }}$ along a particular geotherm. A bin size of $0.2 \mathrm{~km} / \mathrm{s}$ was chosen to represent the minimum error associated with a well constrained seismic experiment [e.g., Korenaga 
et al., 2000], although in reality the error associated with many lower crustal velocity measurements may be significantly higher due to poor ray coverage in the lower crust and the tradeoff between velocity and crustal thickness. Because $V_{p}$ is a function of $P_{r e f}$ and $T_{r e f}$ it is necessary to assess the bulk compositions consistent with each velocity at specific depth intervals along a geotherm. Tables A.1, A.2, and A.3 give the mean and standard deviation in weight percent of the major element oxides for each velocity bin along a normal, cold, and warm crustal geotherm, respectively. Because our synthetic composition space is not weighted by the frequency of naturally occurring bulk compositions it is important to remember that the values in Tables A.1-A.3 represent compositional bounds only, and the mean values do not necessarily reflect the average bulk composition associated with a specific velocity in the lower crust.

The first observation that can be made from Tables A.1-A.3 is that the range of bulk compositions consistent with a given $V_{p}$ is quite large. This implies that without additional information, seismic measurements alone will seldom be sufficient to distinguish between competing models for lower crustal composition. However, while this observation may be discouraging for those hoping to infer compositional information from seismic data, the results compiled in Tables A.1-A.3 indicate that $V_{p}$ can be used to place some useful constraints on bulk composition. For example, Figure 6-9 illustrates the variations in major element oxide composition as a function of $V_{p}$ at a depth of $35 \mathrm{~km}$ along a typical crustal geotherm. With the exception of FeO, all the major element oxides vary as a function of $V_{p}$. The transition from low-velocity, felsic compositions to high-velocity, mafic compositions is clearly seen in the negative correlation of $V_{p}$ to mean $\mathrm{SiO}_{2}$ content and the positve correlation of $V_{p}$ to mean $\mathrm{MgO}$ and $\mathrm{CaO}$ content. Moreover, the variability in $\mathrm{MgO}$ and $\mathrm{CaO}$ content increases steadily with increasing $V_{p}$, due to the trade-off in $\mathrm{MgO}$ and $\mathrm{CaO}$ content in many high velocity mineral phases (e.g., clinopyroxene and orthopyroxene have similar seismic velocities but vary in their $\mathrm{MgO}$ and $\mathrm{CaO}$ content, as do olivine and garnet).

Another important change in major element chemistry occurs at low velocities $(6.3<$ $V_{p}<6.6 \mathrm{~km} / \mathrm{s}$ ), where mean $\mathrm{SiO}_{2}$ and $\mathrm{K}_{2} \mathrm{O}$ content decrease and $\mathrm{Al}_{2} \mathrm{O}_{3}$ and $\mathrm{Na}_{2} \mathrm{O}$ content increase with higher velocity. These variations are associated with the transition from 

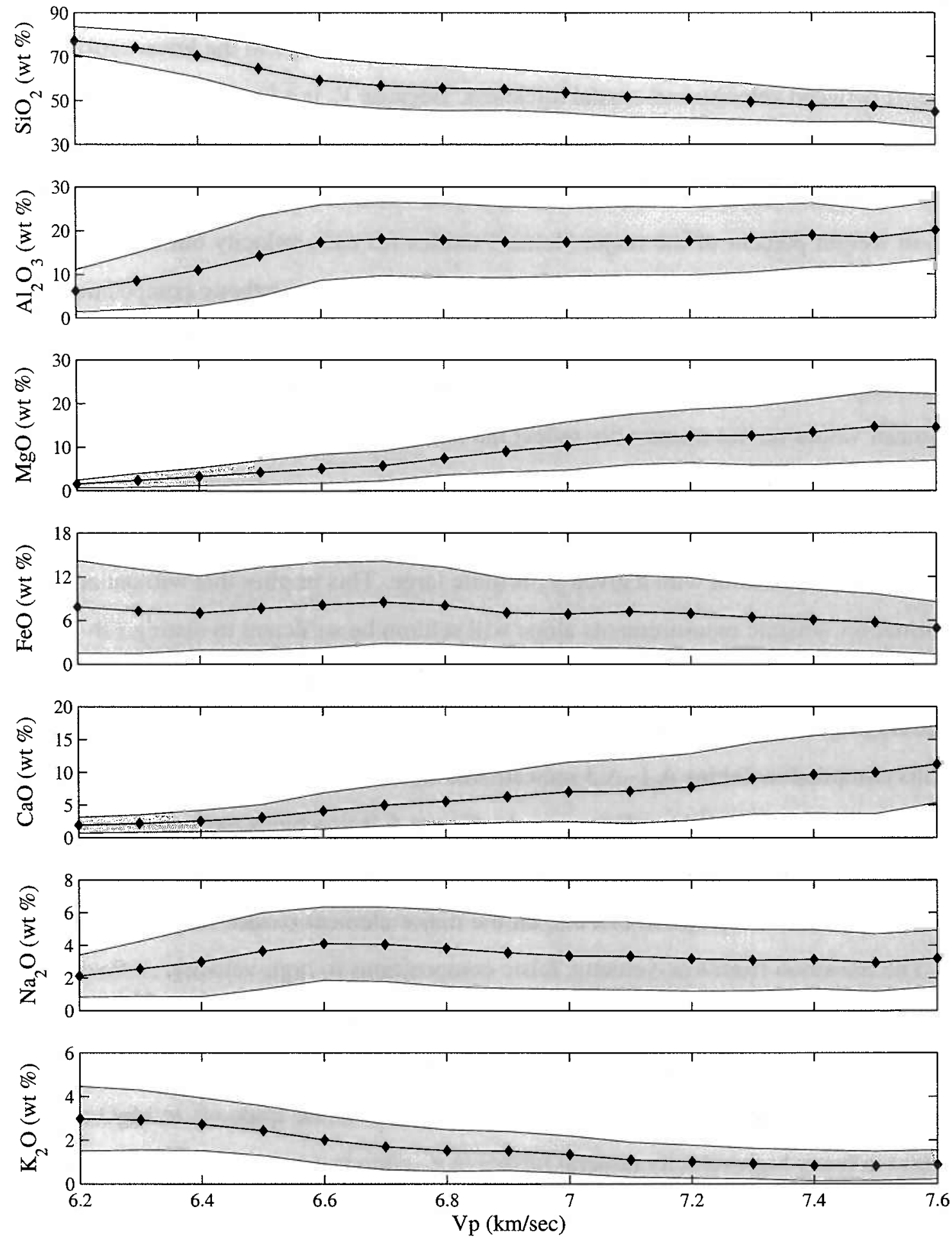

Figure 6-9: Composition bounds for major element oxides as a function of $V_{p}$ for a depth of $35 \mathrm{~km}$ along a normal crustal geotherm. Mean oxide values are denoted by solid diamonds and grey fields represent $1 \sigma$ variation around the mean. Values are taken from Table A.1. 

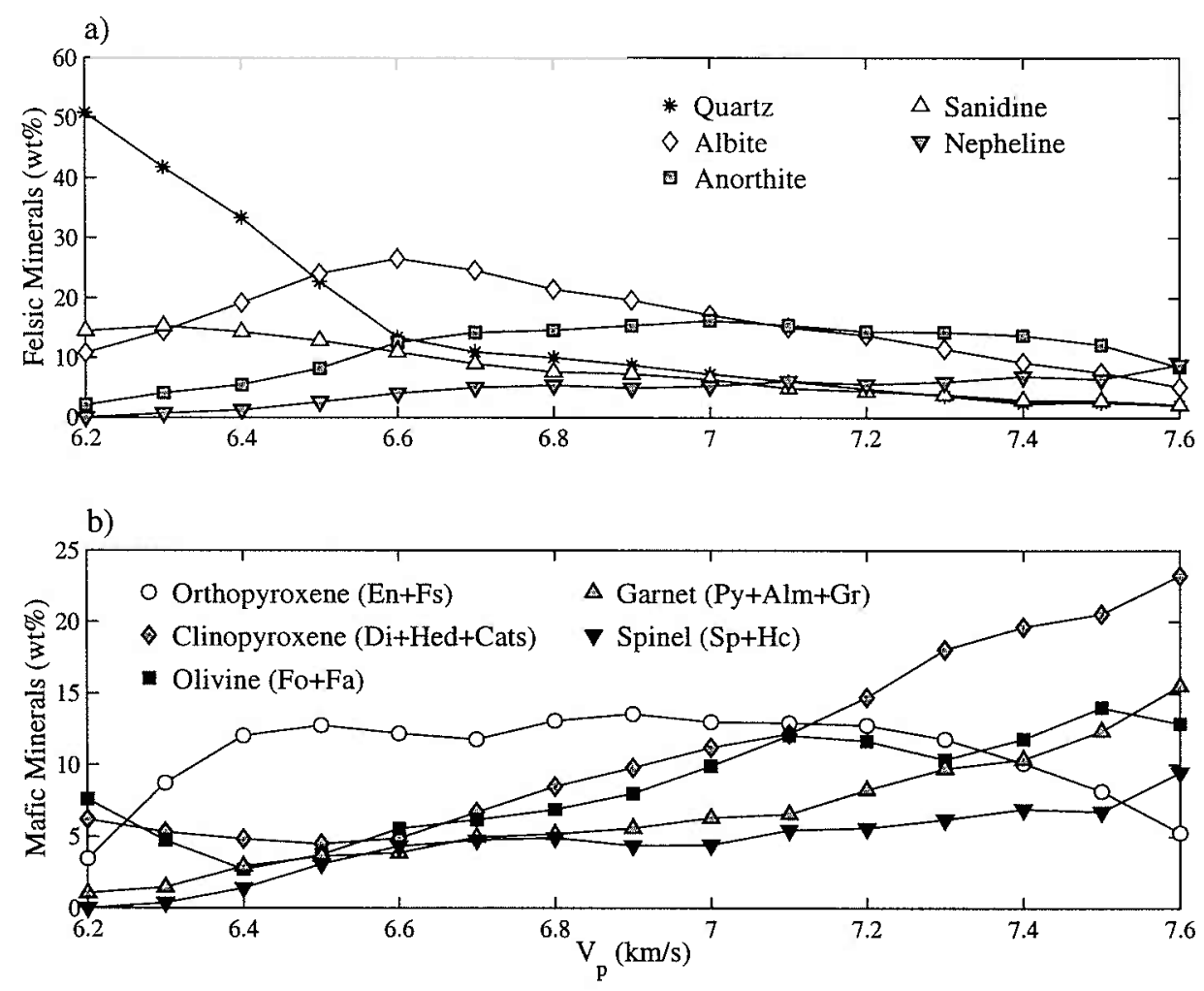

Figure 6-10: Mean weight percent of major a) felsic and b) mafic mineral phases as a function of $V_{p}$ for a depth of $35 \mathrm{~km}$ along a normal crustal geotherm.

granite- to diorite-dominated lithologies, in which quartz $\left(V_{p} \approx 6.0 \mathrm{~km} / \mathrm{s}\right)$ and alkali feldspar $\left(\mathrm{Or}_{54}, V_{p} \approx 5.9 \mathrm{~km} / \mathrm{s}\right)$ are replaced by plagioclase $\left(\mathrm{An}_{53}, V_{p} \approx 6.6 \mathrm{~km} / \mathrm{s}\right)(\mathrm{see}$ Figure 6-10). The abruptness of these compositional changes is significant because it indicates that for velocities between 6.3 to $6.6 \mathrm{~km} / \mathrm{s}$, seismic observations can be used to place distinctive bounds on bulk composition.

\subsection{Discussion}

Applying the relationships developed in the previous section to seismic data from a number of tectonic regimes, we now discuss the implications for the composition of the lower continental and oceanic crust. 


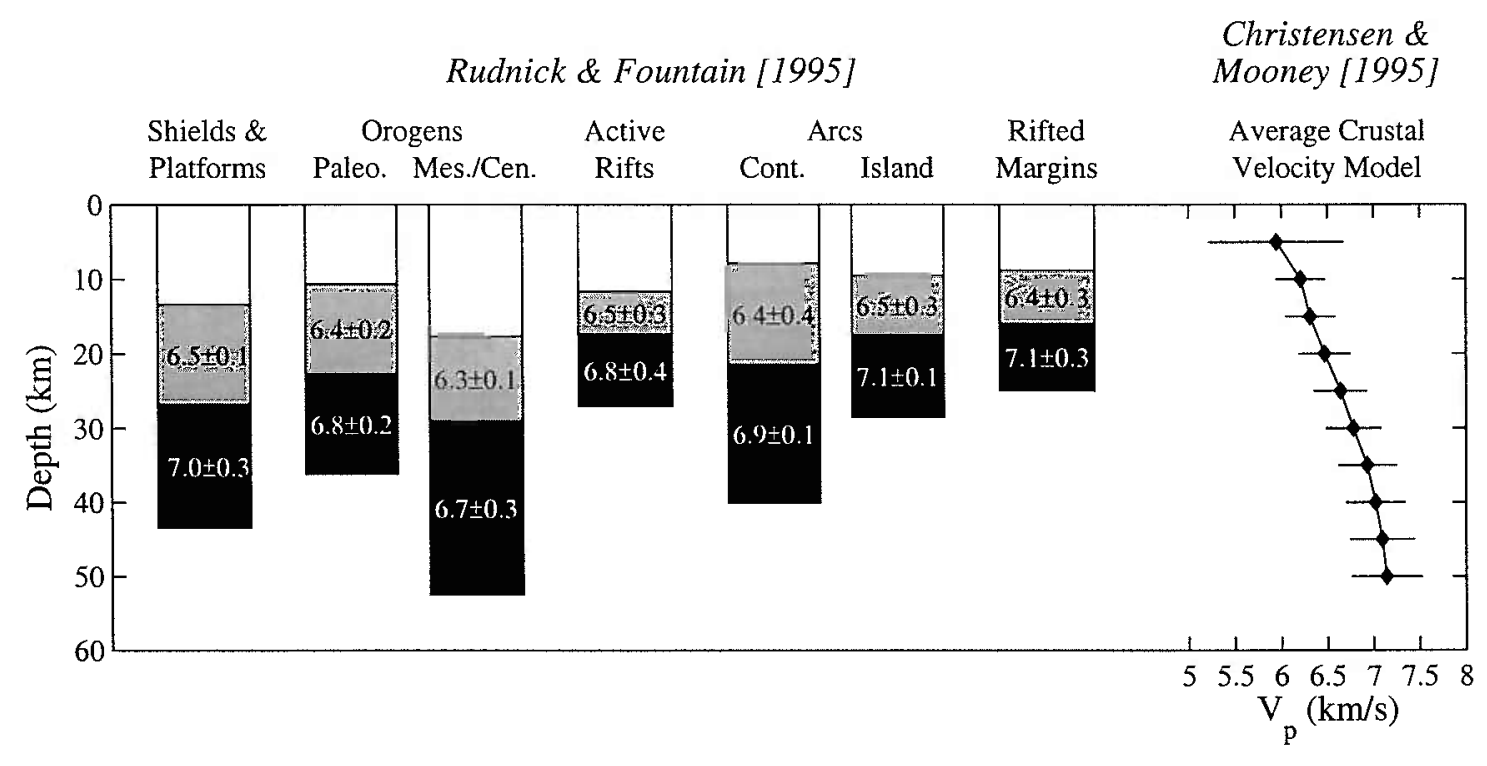

Figure 6-11: Typical crustal velocity sections for a number of continental environments based on the compilation of Rudnick and Fountain [1995] and supplemented with several recent studies from arcs [Suyehiro et al., 1996; Morozov et al., 1998; Fliedner and Klemperer, 1999; Holbrook et al., 1999; Fliedner and Klemperer, 2000] and rifted margins [Korenaga et al., 2000; Holbrook et al., 2001]. Variability represents the standard deviation of all studies in each environment. Also shown is the average crustal velocity model of Christensen and Mooney [1995].

\subsubsection{Lower continental crust}

Figure 6-11 illustrates average crustal velocity sections for a number of continental environments, including Precambrian shields and platforms, orogenic belts, active rifts, continental and island arcs, and rifted margins. These type sections are based on the compilation of Rudnick and Fountain [1995] and are supplemented with several recent studies from arcs [Suyehiro et al., 1996; Morozov et al., 1998; Fliedner and Klemperer, 1999; Holbrook et al., 1999; Fliedner and Klemperer, 2000] and rifted margins [Korenaga et al., 2000; Holbrook et al., 2001]. Also shown for comparison is the average crustal velocity model of Christensen and Mooney [1995], which represents a global average of all continental environments. In general, velocities in the mid-crust average $\sim 6.4 \mathrm{~km} / \mathrm{s}$, while lower crustal velocities range from 6.7 to $7.1 \mathrm{~km} / \mathrm{s}$. Using these models we now calculate constraints on the composition space of the lower continental crust and compare these bounds to previous estimates of lower crustal composition. 
Table 6.4: Compositional estimates for the lower continental, arc, and rifted margin crust. $V_{p}$ is calculated from the major element oxides using Equation 6.4 (valid for $P_{r e f}$ and $T_{\text {ref }}$ conditions along a normal crustal geotherm in the depth range $5-50 \mathrm{~km}$ ). Note that the volcanic margin composition, $B 94$, is an estimated cumulate gabbro composition. Abbreviations are D79 [Dupuy et al., 1979], WT84 [Weaver and Tarney, 1984], TM85 [Taylor and McLennan, 1985], S86 [Shaw et al., 1986], RP90at [Archean terranes; Rudnick and Presper, 1990], RP90x [Xenoliths; Rudnick and Presper, 1990], W90 [Correct reference from Peter], RF95 [Rudnick and Fountain, 1995], B83 [Burns, 1983], DS91 [DeBari and Sleep, 1991], and B94 [Bernstein, 1994].

\begin{tabular}{|l|ccccccccccc|c|}
\multicolumn{10}{c|}{} & \multicolumn{10}{c|}{ Lower Continental } & \multicolumn{1}{c|}{ Volcanic } \\
\cline { 2 - 12 } \multicolumn{1}{c|}{} & $D 79$ & WT84 & $T M 85$ & $S 86$ & RP90at & $R P 90 x$ & W90 & $R F 95$ & $B 83$ & $D S 91$ & $B 94$ \\
\hline $\mathrm{SiO}_{2}$ & 56.3 & 61.2 & 54.4 & 61.5 & 63.3 & 50.5 & 60.0 & 52.3 & 45.7 & 43.8 & 48.49 \\
$\mathrm{TiO}_{2}$ & 1.1 & 0.5 & 1.0 & 0.8 & 0.6 & 1.0 & 0.8 & 0.8 & 0.9 & 0.7 & 0.61 \\
$\mathrm{Al}_{2} \mathrm{O}_{3}$ & 17.1 & 15.6 & 16.1 & 14.9 & 14.6 & 16.5 & 15.3 & 16.6 & 19.3 & 18.7 & 15.87 \\
$\mathrm{FeO}$ & 7.9 & 5.3 & 10.6 & 7.2 & 5.6 & 9.0 & 7.0 & 8.4 & 11.5 & 10.2 & 5.12 \\
$\mathrm{MnO}$ & 0.1 & 0.1 & - & 0.1 & 0.1 & 0.2 & 0.1 & 0.1 & 0.2 & 0.2 & 0.13 \\
$\mathrm{MgO}$ & 5.0 & 3.4 & 6.3 & 4.1 & 3.5 & 7.7 & 4.6 & 7.1 & 7.7 & 10.6 & 12.52 \\
$\mathrm{CaO}$ & 5.5 & 5.6 & 8.5 & 5.2 & 5.1 & 9.8 & 6.1 & 9.4 & 12.9 & 14.0 & 16.64 \\
$\mathrm{Na}_{2} \mathrm{O}$ & 2.1 & 4.4 & 2.8 & 3.1 & 3.4 & 2.4 & 3.0 & 2.6 & 1.7 & 1.7 & 0.50 \\
$\mathrm{~K}_{2} \mathrm{O}$ & 1.4 & 1.0 & 0.3 & 1.6 & 2.2 & 0.8 & 1.8 & 0.6 & 0.1 & 0.1 & 0.10 \\
$\mathrm{P}_{2} \mathrm{O}_{5}$ & 0.2 & 0.2 & - & 0.1 & 0.2 & 0.2 & 0.2 & 0.1 & 7.2 & 0.1 & - \\
\hline$V_{p}(\mathrm{~km} / \mathrm{s})$ & 6.7 & 6.6 & 6.9 & 6.6 & 6.6 & 7.0 & 6.7 & 7.0 & 7.2 & 7.4 & 7.5 \\
\hline
\end{tabular}

The average bulk composition of the lower continental crust has been estimated using a variety of techniques, including rare earth element patterns [Taylor and McLennan, 1981, 1985], the chemical composition of exposed granulite terranes [Shaw et al., 1986; Rudnick and Presper, 1990], xenoliths from volcanic and kimberlite eruptions [Dupuy et al., 1979; Kay and Kay, 1981; Rudnick and Presper, 1990], heat flow [Taylor and McLennan, 1985; McLennan and Taylor, 1996] and seismic data [Rudnick and Fountain, 1995]. Several of these estimates are listed Table 6.4, along with corresponding $V_{p}$ values calculated from the major element oxides using Equation 6.4. The calculated velocities range from $6.6-7.0 \mathrm{~km} / \mathrm{s}$ and are in good agreement with the observed velocities for the lower continental crust (Figure 6-11).

Using the average crustal velocity model of Christensen and Mooney [1995] we calculated compositional bounds for each major element oxide as a function of depth (Table 6.5), following a similar procedure as that used to generate Tables A.1-A.3. A normal 


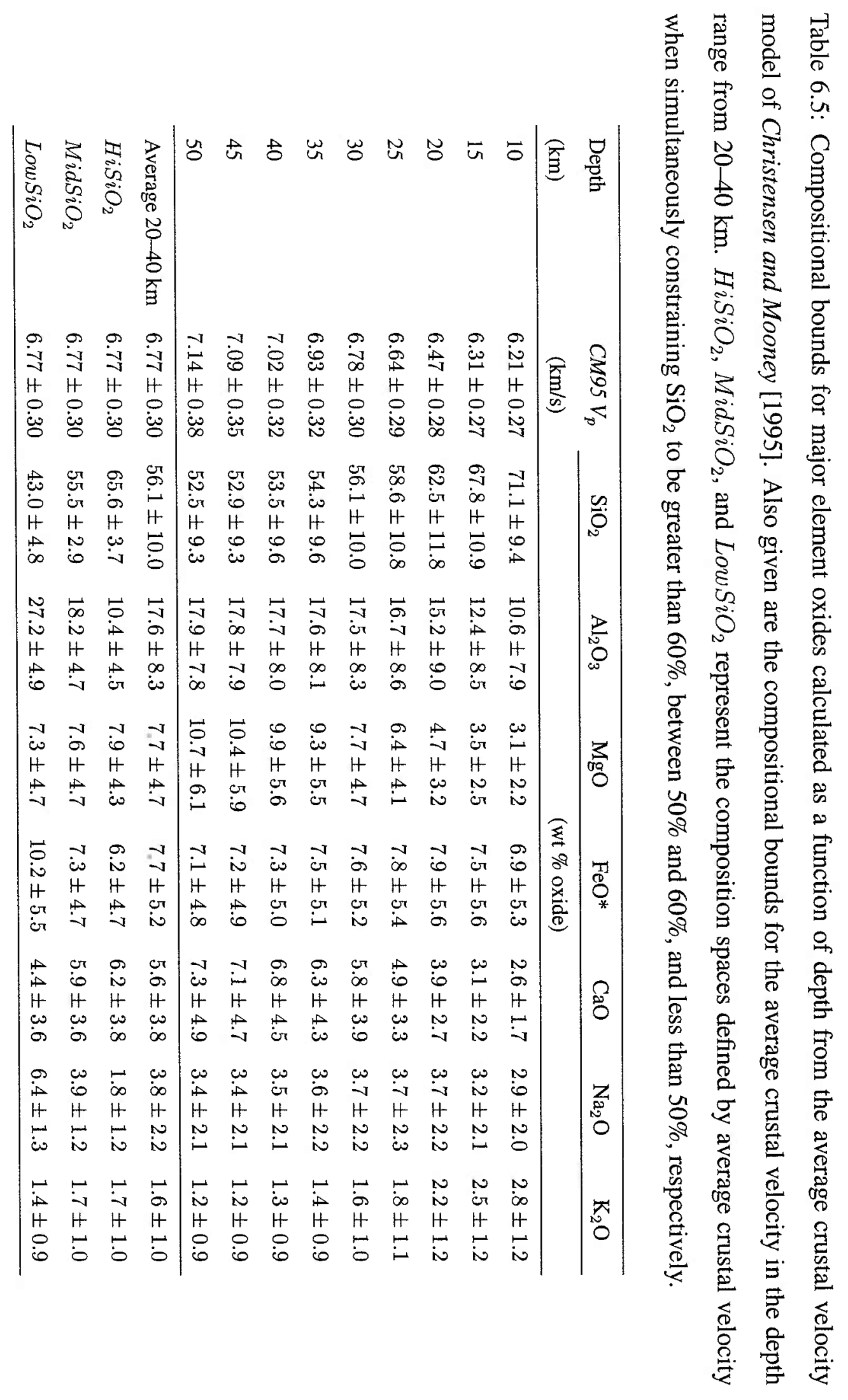


geotherm is assumed and the synthetic composition space is searched at each depth interval in a $V_{p}$ bin defined by the Christensen and Mooney [1995] model. The resulting composition space for the lower continental crust is large, and most of the compositional estimates presented in Table 6.4 fall well within the permissible limits. This is important because several recent studies suggest that only a basaltic lower crustal composition (e.g., $R P 90 x$ in Table 6.4) is consistent with the "high" lower crustal $V_{p}$ in the Christensen and Mooney [1995] average crustal velocity model. However, the results of this analysis clearly show that a more granitic composition is not excluded based on seismic velocities alone.

In reality, the large range of acceptable crustal compositions should not be particularly surprising. The Christensen and Mooney [1995] model is constructed from a global compilation of seismic data encompassing all continental environments. Regional hetrogeneity in lower crustal velocities is large within individual tectonic regimes and is even greater between different tectonic environments. At a depth of $35 \mathrm{~km}$, the Christensen and Mooney [1995] model is characterized by velocities ranging from 6.61 to $7.25 \mathrm{~km} / \mathrm{s}$. Thus, it is striking that several of the compositional estimates in Table 6.4 fall outside the calculated limits. For example, at $35 \mathrm{~km}$ the Weaver and Tarney [1984] and Rudnick and Presper [1990] (Archian terrane) estimates for $\mathrm{MgO}$ content in lower crustal rocks are lower than the calculated bounds, and the $\mathrm{K}_{2} \mathrm{O}$ estimate of Taylor and McLennan [1985] is also below the acceptable limits. Nevertheless, the permissible compositional space for the lower continental crust based on average crustal velocities is too broad to provide any fundamental insights into bulk crustal composition.

On the other hand, seismic inferences of composition can provide more meaningful results when seismic data are used to evaluate compositional variations between individual locations or differences relative to a specific reference model. For example, lower crustal velocities along the western North Atlantic margin are observed to vary from $6.7 \mathrm{~km} / \mathrm{s}$ near the Grand Banks, Newfoundland [Reid, 1994] to $7.5 \mathrm{~km} / \mathrm{s}$ offshore of Virginia [Holbrook et al., 1994a]. These variations have been interpreted to reflect spatial variations in the composition of the lower margin crust and the evolution of the continental margin. To the north, the lower crust is assumed to consist primarily of remnant continental material of 
granitic origin [e.g., Tucholke et al., 1989]. In contrast, the higher velocities observed along the mid-Atlantic margin are typically associated with basaltic and gabbroic compositions formed by syn-rift volcanism and igneous "underplating" [e.g., Holbrook and Kelemen, 1993].

Using the compositional bounds in Table A.1 we assess the differences in composition associated with a change in $V_{p}$ from 6.7 to $7.5 \mathrm{~km} / \mathrm{s}$ at a depth of $20 \mathrm{~km}$. Although the $1 \sigma$ bounds are sufficiently wide that the compositional range for each major element oxide cannot be formally distinguished, the calculated composition spaces are quite different. For example, the range in $\mathrm{SiO}_{2}$ content calculated for $\mathrm{V}_{\mathrm{p}}=6.7 \mathrm{~km} / \mathrm{s}$ is $57 \pm 10 \%$, while for $\mathrm{V}_{\mathrm{p}}=7.5 \mathrm{~km} / \mathrm{s}$ the range is $47 \pm 7 \%$. Similiarly, the range in $\mathrm{MgO}$ content is found to be $6 \pm 4 \%$ for $V_{p}=6.7 \mathrm{~km} / \mathrm{s}$ and $15 \pm 8 \%$ for $V_{p}=7.5 \mathrm{~km} / \mathrm{s}$. Comparing these compositional bounds to the estimates of lower crustal composition presented in Table 6.4, we see that a $V_{p}$ of $7.5 \mathrm{~km} / \mathrm{s}$ is inconsistent with most estimates for the lower continental crust (except $R P 90 x$ and $R F 95$ ) and is most consistent with a cumulate gabbro composition (B94). In contrast, the calculated $\mathrm{MgO}, \mathrm{CaO}$, and $\mathrm{Na}_{2} \mathrm{O}$ content for $\mathrm{V}_{\mathrm{p}}=6.7 \mathrm{~km} / \mathrm{s}$ are not consistent with a cumulate gabbro and resemble more felsic compositions.

It is also important to note that while the compositional bounds associated with a specific $V_{p}$ are large, there is significant covariation within the major element oxides. Thus, in situations where compositional as well as seismic constraints can be placed on the synthetic data space, the range of permissible bulk compositions can be greatly reduced. To illustrate this point, we take the average $V_{p}$ between 20 and $40 \mathrm{~km}$ from the Christensen and Mooney [1995] model and calculate three cases in which $\mathrm{SiO}_{2}$ content is limited to be greater than $60 \%\left(\mathrm{HiSiO}_{2}\right)$, between $50 \%$ and $60 \%\left(\mathrm{MidSiO}_{2}\right)$, and less than $50 \%$ $\left(\mathrm{LowSiO}_{2}\right)$. The resulting compositional bounds are given at the bottom of Table 6.5 and frequency distributions of the major element oxides for the $\mathrm{HiSiO}_{2}$ and $\mathrm{LowSiO}_{2}$ cases are shown in Figure 6-12. In addition to $\mathrm{SiO}_{2}$, significant differences are observed in the compositional bounds for both $\mathrm{Al}_{2} \mathrm{O}_{3}$ and $\mathrm{Na}_{2} \mathrm{O}$ between the three examples. The $\mathrm{HiSiO}_{2}$ composition is a high $\mathrm{Mg}$-number andesite to dacite composition such as is common in calc-alkaline arc lavas and compositionally similar intermediate plutonic rocks character- 
Example: $\mathrm{HiSiO}_{2}$
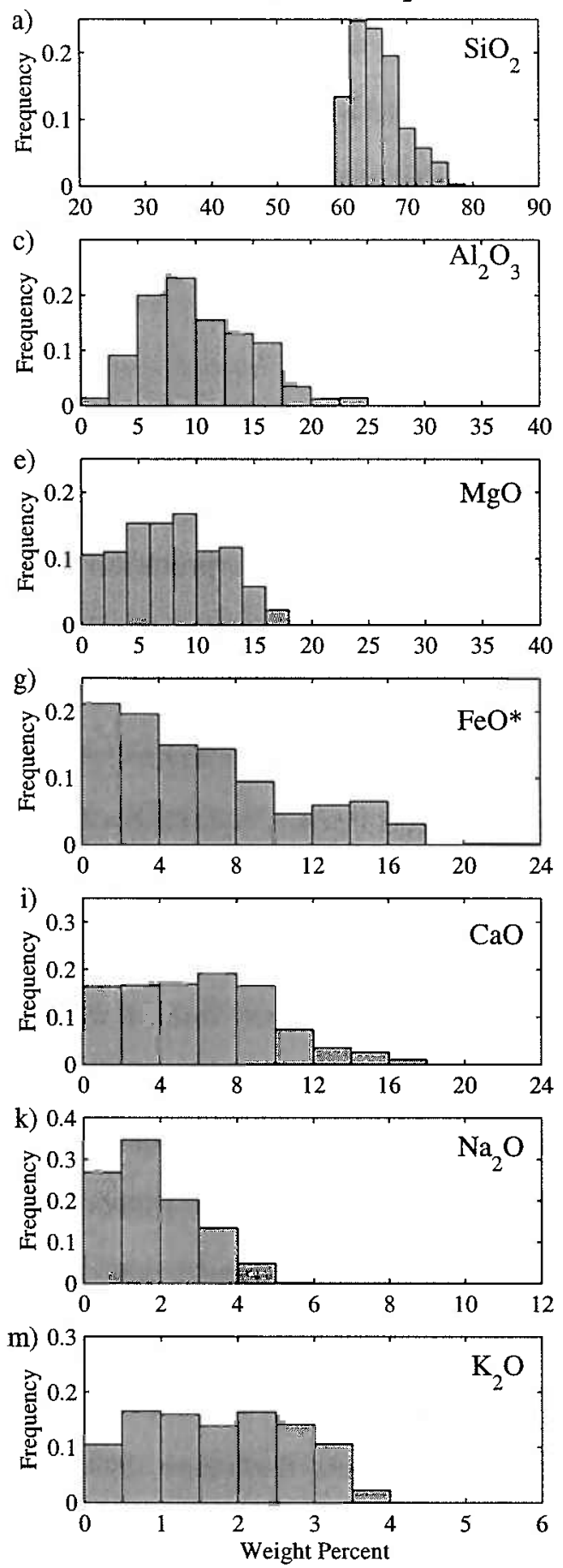

Example: $\mathrm{LowSiO}_{2}$

b)

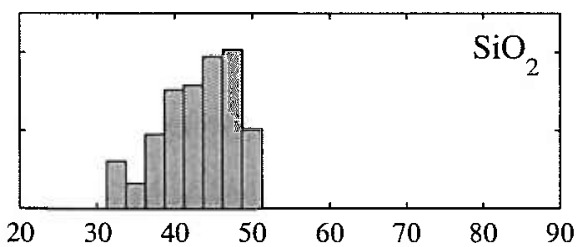

d)

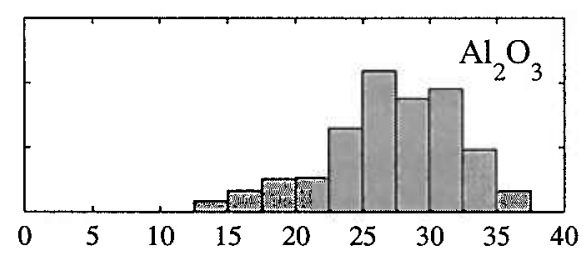

f)

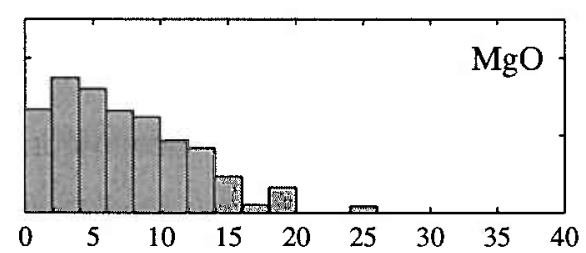

h)

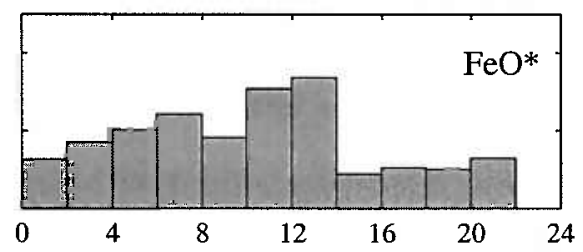

j)

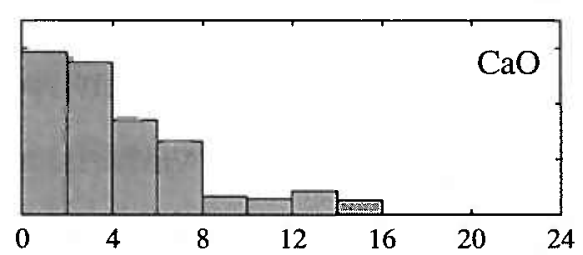

1)

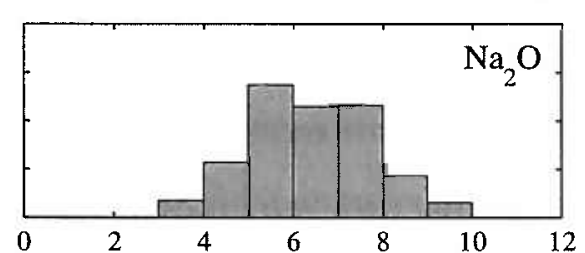

n)

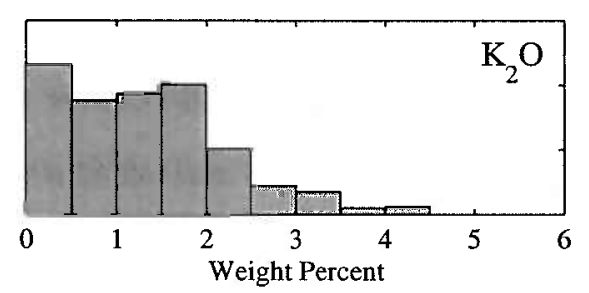

Figure 6-12: Frequency distributions of the major element oxides for examples, $\mathrm{HiSiO}_{2}$ and $\mathrm{LowSiO}_{2}$. Distributions are calculated from the synthetic data by simultaneously constraining $V_{p}$ and $\mathrm{SiO}_{2}$ content. In both cases, $V_{p}$ is constrained using the average crustal velocity between 20 and $40 \mathrm{~km}$ from Christensen and Mooney [1995], while $\mathrm{SiO}_{2}$ content is limited to be $>60 \%$ for $\mathrm{HiSiO}_{2}$ and $<50 \%$ for $\mathrm{LowSiO}_{2}$. 
istic of batholiths. The $\mathrm{MidSiO}_{2}$ example is very similar to the composition predicted for the average $V_{p}$ with no $\mathrm{SiO}_{2}$ constraint. Finally, the $\mathrm{LowSiO}_{2}$ composition, while somewhat similar to observed lower crustal compositions in island arc sections (B83, DS9l in Table 6.4), is a very unlikely natural composition due to its extremely high $\mathrm{Al}_{2} \mathrm{O}_{3}$ content and low $\mathrm{CaO}$ content.

\subsubsection{Lower oceanic crust}

We now examine the implications of this study for the lower oceanic crust. The classic model for the composition of oceanic crust is based on the joint interpretation of ophiolite sequences and seismic observations at modern oceanic spreading centers. In this model the lower oceanic crust is composed of a 4-5.5 km thick section of massive and cumulate gabbros. This section, often referred to in seismic studies as oceanic layer 3, is characterized by a low velocity gradient with depth and an average $V_{p}$ of $6.9-7.0 \mathrm{~km} / \mathrm{s}$ [Raitt, 1963; Shor et al., 1970; Christensen and Salisbury, 1975; White et al., 1992]. Deviations from this mean value have typically been interpreted to reflect anomalous crustal compositions formed in the presence of elevated mantle temperatures [e.g., White and McKenzie, 1989; Kelemen and Holbrook, 1995]. Using the melting model of McKenzie and Bickle [1988], White and McKenzie [1989] found that if normal oceanic crust is assumed to form from a mantle potential temperature of $1280^{\circ} \mathrm{C}$, then a potential temperature of $1580^{\circ} \mathrm{C}$ is required to generate crust with velocities of $7.2 \mathrm{~km} / \mathrm{s}$. This result has subsequently been used to infer that the thick sections of high velocity lower crust $\left(V_{p} \geq 7.2 \mathrm{~km} / \mathrm{s}\right)$ observed at a number of volcanic rifted margins and oceanic plateaus must have formed in the presence of a hot mantle plume [e.g. White and McKenzie, 1989; Kelemen and Holbrook, 1995; Holbrook et al., 2001].

Estimates for the average bulk composition of the lower oceanic crust have been made from gabbros in dredged samples [Hyndman, 1985; Hekinian et al., 1993], drill cores [Pederson et al., 1996; Casey, 1997; Dick et al., 2000], and exposed ophiolite sequences [Browning, 1982; Pallister, 1984] (Table 6.6). Based on these estimates we calculate pre- 
Table 6.6: Compositional estimates for the lower oceanic crust from gabbros in exposed ophiolite sequences, drill cores, and dredged samples. $V_{p}$ is calculated from the major element oxides using Equation 6.4 and the regression coefficients derived for a warm geotherm (Table 6.3). Abbreviations are B82 [Browning, 1982], P84 [Pallister, 1984], KGunpub [Kelemen and Garrido, unpublished data from Wadi Tayin massif, Oman ophiolite], MARK [ODP Leg 153, Casey, 1997], Hess [ODP Leg 147, Pederson et al., 1996; Miller et al., 1996, plus 3 dredge samples from Hekinian et al., 1993], 735B [ODP Leg 176, Dick et al., 2000], and H85 [Hyndman, 1985].

\begin{tabular}{|l|ccc|ccc|c|}
\multicolumn{1}{c}{} & \multicolumn{3}{c}{ Ophiolite } & \multicolumn{3}{c}{ Drill Core } & Dredge \\
\cline { 2 - 8 } \multicolumn{1}{c|}{} & $B 82$ & P84 & KGunpub & MARK & Hess & $735 B$ & H85 \\
\hline $\mathrm{SiO}_{2}$ & 52.0 & 51.1 & 48.4 & 49.5 & 50.2 & 50.6 & 50.2 \\
$\mathrm{TiO}_{2}$ & 0.6 & 0.6 & 0.3 & 0.7 & 0.7 & 0.9 & 0.3 \\
$\mathrm{Al}_{2} \mathrm{O}_{3}$ & 13.5 & 16.6 & 18.4 & 17.8 & 15.2 & 16.1 & 16.2 \\
$\mathrm{FeO}$ & 7.2 & 7.2 & 5.2 & 6.3 & 9.1 & 7.3 & 5.7 \\
$\mathrm{MnO}$ & 0.1 & 0.1 & 0.1 & 0.1 & 0.2 & 0.1 & 0.1 \\
$\mathrm{MgO}$ & 13.5 & 9.2 & 10.3 & 9.8 & 9.2 & 9.2 & 10.0 \\
$\mathrm{CaO}$ & 10.9 & 12.8 & 15.8 & 12.3 & 12.1 & 12.5 & 14.0 \\
$\mathrm{Na}_{2} \mathrm{O}$ & 2.2 & 2.3 & 1.4 & 2.5 & 2.3 & 2.8 & 1.7 \\
$\mathrm{~K}_{2} \mathrm{O}$ & 0.1 & 0.1 & 0.0 & 0.0 & 0.1 & 0.1 & 0.1 \\
$\mathrm{P}_{2} \mathrm{O}_{5}$ & 0.0 & 0.1 & 0.0 & 0.1 & 0.1 & 0.1 & 0.0 \\
\hline$V_{p}(\mathrm{~km} / \mathrm{s})($ Eq. 6.4) & 7.3 & 7.2 & 7.4 & 7.2 & 7.2 & 7.2 & 7.3 \\
$V_{p}(\mathrm{~km} / \mathrm{s})($ Warm) & 7.2 & 7.1 & 7.3 & 7.1 & 7.1 & 7.1 & 7.2 \\
\hline
\end{tabular}

dicted velocities of $7.1-7.3 \mathrm{~km} / \mathrm{s}$ for the lower oceanic crust-significantly higher than the average observed value for oceanic layer 3 and very similar to lower crustal $V_{p}$ in volcanic rifted margins. Because most seismic velocity measurements in oceanic crust are made at or near the ridge crest, the regression coefficients derived for the warm geotherm (Table 6.3) were used to simulate thermal conditions in 5-10 Ma oceanic lithosphere [Stein and Stein, 1992]. Had the coefficients for the normal geotherm in Equation 6.4 been used, the predicted velocities would be higher by $\sim 0.1 \mathrm{~km} / \mathrm{s}$ (Table 6.6 ).

These results are significant because they indicate that in situ measurements of $V_{p}$ at oceanic spreading centers are $0.2-0.3 \mathrm{~km} / \mathrm{s}$ slow compared to the velocities predicted for gabbro. Korenaga et al. [2002] also observed this discrepancy and suggested two possible mechanisms for the slow velocities in oceanic layer 3: 1) alteration associated with hydrothermal circulation, and 2) residual crack porosity. Assuming that the effect of hy- 
drothermal alteration can be approximated by replacement of clinopyroxene with hornblende, Korenaga et al. [2002] calculated that even 100\% alteration is unable to reduce $V_{p}$ to $6.9 \mathrm{~km} / \mathrm{s}$ in typical oceanic gabbros. Thus, they favored a model in which the slow velocities in the lower crust are the result of residual porosity. Specifically, Korenaga et al. [2002] noted that if the aspect ratio of pores is low (i.e., crack-shaped), porosities of $<1 \%$ are necessary to reduce velocities to $6.9 \mathrm{~km} / \mathrm{s}$. These estimates of bulk porosity are in agreement with measurements from ODP Hole 735B [Iturrino et al., 1991] and the Continental Deep Drilling Project (KTB) [Huenges et al., 1997], which show porosities of 0.5-1\% at depths approaching $4 \mathrm{~km}$.

Experimental data for $V_{p}$ in gabbros also support the hypothesis that residual porosity may be the cause of the apparently low velocities in oceanic layer 3 compared to calculated velocities for likely lower crustal gabbro compositions. Figure 6-13 illustrates $V_{p}$ versus pressure in gabbro samples taken from ODP drill cores at Hess Deep [Iturrino et al., 1996] and the MARK Area [Miller and Christensen, 1997] and ophiolites in the western United States [Christensen, 1978]. For pressures greater than $2 \mathrm{kbar}$, the change in $V_{p}$ with pressure follows closely the predicted value based on the compressibilities of typical mafic minerals. However, from $0-2 \mathrm{kbar}$ the change in $V_{p}$ with pressure is significantly greater, suggesting that the complete closure of cracks is not achieved the at ambient pressures present in the lower oceanic crust. This conclusion is consistent with earlier experimental studies by Kern and Richter [1981] and Kern and Schenk [1985, 1988] and also with the comparison of the best-fit $V_{p}$ to the laboratory data presented in Figure 6-6.

A final explanation for the discrepancy between the measured layer 3 velocities and those calculated for likely lower crustal gabbro compositions is that the lower crust is composed of partially serpentinized mantle peridotites. This model for the lower oceanic crust was originally proposed by Hess [1962, 1965] and Dietz [1963]. However, a recent study by Carlson and Miller [1997] found that the observed S-wave velocities in the lower crust are more compatible with gabbroic compositions than partially serpentinized peridotites. Nonetheless, serpentinized peridotites are commonly found in slow-spreading environments and their presence in small quantities can strongly effect $V_{p}$ [Carlson, 2001]. 


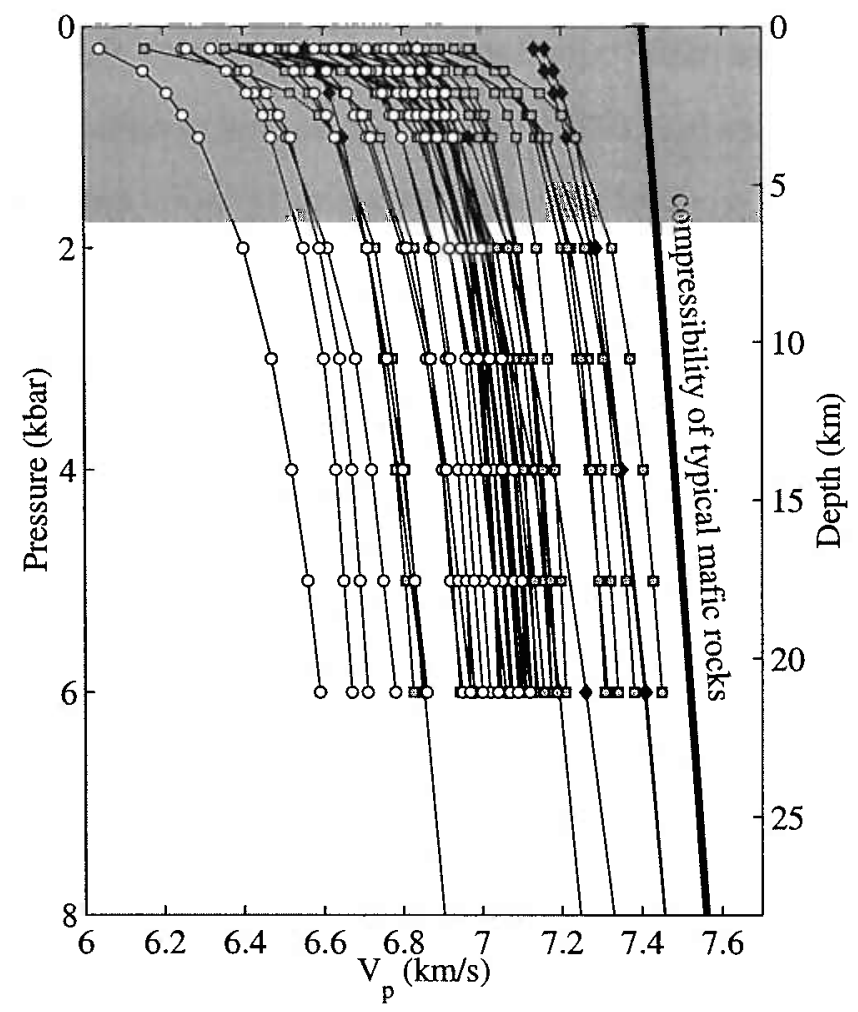

Figure 6-13: Laboratory measurements of $V_{p}$ as a function of confining pressure in gabbros taken from ODP Leg 147, Hess Deep (circles) [Iturrino et al., 1996], ODP Leg 153, MARK Area (squares) [Miller and Christensen, 1997], and ophiolite exposures in the western United States (diamonds) [Christensen, 1978]. All measurements were made at room temperature. Thick solid line illustrates a typical pressure derivative for mafic rocks due to the effect of pressure on the elastic moduli of major mineral phases. Note that at confining pressures appropriate for the oceanic crust (grey region) the change in $V_{p}$ with pressure is significantly greater than that predicted from the mineral compressibilities alone. 
Thus, they may be at least partially responsible for the slow observed velocities in the lower oceanic crust.

The observation that the lower oceanic crust is slow relative to the velocity predicted for the bulk composition of gabbro has several important implications. First, it suggests that the observed velocity gradients in layer 3 are associated with changes in pressure and not a transition toward more mafic cumulate compositions [e.g., Salisbury and Christensen, 1984; Christensen and Smewing, 1981]. Second, if normal oceanic gabbros have velocities of $7.1-7.3 \mathrm{~km} / \mathrm{s}$, then the sections of "high" velocity igneous crust observed in the thick lower crust at many volcanic margins and oceanic plateaus may not reflect anomalous lower crustal composition. Rather, it may be that the increase in ambient pressure at the base of the thickened crust at these margins is sufficient to close all cracks. This would imply that elevated mantle temperatures are not required for the formation of these thick igneous sequences, and that alternative models in which large amounts of melt are fluxed through the solidus in the absence of a thermal anomaly [e.g., Mutter et al., 1988] must be reevaluated.

\subsection{Conclusions}

In this study we have presented a new method to quantitatively assess the relationship between the composition and seismic velocity of igneous rocks. Using seismic velocities calculated from equilibrium mineral assemblages for all igneous composition space, we derived a linear function relating $V_{p}$ to major element chemistry that is valid to $\pm 0.12 \mathrm{~km} / \mathrm{s}$ for all pressure and temperature conditions along a normal crustal geotherm in the depth range of $5-50 \mathrm{~km}$. We note that the equilibration calculations performed in this study ignore the presence of both $\mathrm{Fe}^{3+}$ and $\mathrm{H}_{2} \mathrm{O}$ and future work is needed to assess the influence of these variables on the relationship between $V_{p}$ and composition.

The calculated mineral assemblages were also used to address the inverse problem, namely what is the full range of igneous compositions that are consistent with a particular seismic velocity. The resulting compositional bounds for a specific velocity were found 
to be extremely large. For example, in the case of continental crust, we find that average velocity-depth profiles cannot be used to distinguish between basaltic and granitic lower crustal compositions. Thus, we conclude that while composition can be used to provide a robust estimate of $V_{p}$, seismic velocities alone cannot provide a robust estimate of bulk crustal composition.

Finally, based on the forward relationship between $V_{p}$ and major element chemistry derived in this study, we find the observed velocities in the lower oceanic crust to be $0.2-0.3$ $\mathrm{km} / \mathrm{s}$ slower than the velocities predicted based on the average bulk composition of gabbros in drill cores and exposed ophiolite sequences. We hypothesize that this discrepancy is caused by microcracks that are not fully closed at ambient pressures in the lower oceanic crust. If correct, this implies that future studies should use caution when inferring mantle melting parameters from lower crustal velocities at depths shallower than $\sim 10 \mathrm{~km}$. 


\section{Appendix A}

\section{Compositional Tables}


Table A.1: Mean and standard deviation of major element oxides as a function of $V_{p}$ for a normal continental geotherm $\left(q_{s}=56 \mathrm{mWm}^{-2}\right) . N$ denotes number of synthetic compositions in each $V_{p}$ bin.

\begin{tabular}{|c|c|c|c|c|c|c|c|c|c|c|}
\hline Depth $(\mathrm{km})$ & 5 & 10 & 15 & 20 & 25 & 30 & 35 & 40 & 45 & 50 \\
\hline $\mathrm{T}(\mathrm{C})$ & 90.90 & 154.56 & 210.78 & 262.50 & 311.49 & 358.82 & 405.15 & 450.86 & 496.21 & 541.33 \\
\hline $\mathrm{P}$ (kbar) & 1.62 & 3.23 & 4.85 & 6.47 & 8.09 & 9.70 & 11.32 & 12.94 & 14.55 & 16.17 \\
\hline \multicolumn{11}{|c|}{$V_{p}=6.2 \mathrm{~km} / \mathrm{s}$} \\
\hline Mean & & & & & & & & & & \\
\hline $\mathrm{SiO}_{2}$ & 75.88 & 76.34 & 76.33 & 76.75 & 76.97 & 77.46 & 77.29 & 75.94 & 76.62 & 77.05 \\
\hline $\mathrm{Al}_{2} \mathrm{O}_{3}$ & 7.47 & 7.11 & 7.11 & 6.85 & 6.68 & 6.04 & 6.26 & 7.45 & 7.09 & 6.91 \\
\hline $\mathrm{MgO}$ & 2.16 & 2.10 & 2.10 & 2.06 & 2.06 & 1.89 & 1.54 & 1.19 & 0.97 & 0.94 \\
\hline $\mathrm{FeO}$ & 7.23 & 7.32 & 7.32 & 7.38 & 7.40 & 7.80 & 7.88 & 9.39 & 9.29 & 9.11 \\
\hline $\mathrm{CaO}$ & 2.01 & 2.00 & 1.99 & 1.88 & 1.86 & 1.88 & 1.93 & 1.40 & 1.49 & 1.51 \\
\hline $\mathrm{Na}_{2} \mathrm{O}$ & 2.32 & 2.22 & 2.23 & 2.18 & 2.13 & 2.02 & 2.12 & 2.22 & 2.12 & 2.05 \\
\hline $\mathrm{K}_{2} \mathrm{O}$ & 2.94 & 2.92 & 2.93 & 2.92 & 2.91 & 2.92 & 2.98 & 2.42 & 2.43 & 2.43 \\
\hline \multicolumn{11}{|l|}{ Std. Dev. } \\
\hline $\mathrm{SiO}_{2}$ & 6.32 & 6.52 & 6.55 & 6.24 & 6.22 & 5.88 & 6.37 & 6.64 & 7.70 & 7.67 \\
\hline $\mathrm{Al}_{2} \mathrm{O}_{3}$ & 5.30 & 5.28 & 5.30 & 5.06 & 5.01 & 4.64 & 4.86 & 5.40 & 5.57 & 5.59 \\
\hline $\mathrm{MgO}$ & 1.14 & 1.11 & 1.12 & 1.13 & 1.14 & 1.04 & 0.91 & 0.75 & 0.54 & 0.54 \\
\hline $\mathrm{FeO}$ & 5.50 & 5.49 & 5.51 & 5.59 & 5.50 & 5.83 & 6.34 & 6.86 & 7.20 & 6.92 \\
\hline $\mathrm{CaO}$ & 1.13 & 1.14 & 1.14 & 1.14 & 1.14 & 1.10 & 1.23 & 0.85 & 0.83 & 0.84 \\
\hline $\mathrm{Na}_{2} \mathrm{O}$ & 1.38 & 1.40 & 1.40 & 1.36 & 1.36 & 1.29 & 1.29 & 1.51 & 1.55 & 1.56 \\
\hline $\mathrm{K}_{2} \mathrm{O}$ & 1.30 & 1.31 & 1.31 & 1.32 & 1.31 & 1.37 & 1.48 & 1.14 & 1.01 & 0.97 \\
\hline$N$ & 150 & 147 & 146 & 141 & 135 & 107 & 74 & 47 & 34 & 32 \\
\hline \multicolumn{11}{|c|}{$V_{p}=6.3 \mathrm{~km} / \mathrm{s}$} \\
\hline Mean & & & & & & & & & & \\
\hline $\mathrm{SiO}_{2}$ & 73.54 & 73.65 & 73.55 & 73.79 & 73.94 & 73.94 & 74.13 & 74.37 & 75.26 & 75.43 \\
\hline $\mathrm{Al}_{2} \mathrm{O}_{3}$ & 8.71 & 8.80 & 8.67 & 8.39 & 8.36 & 8.59 & 8.50 & 8.45 & 7.98 & 7.92 \\
\hline $\mathrm{MgO}$ & 3.02 & 2.97 & 2.86 & 2.89 & 2.83 & 2.56 & 2.40 & 2.03 & 2.08 & 2.04 \\
\hline $\mathrm{FeO}$ & 6.76 & 6.57 & 7.00 & 7.23 & 7.08 & 7.05 & 7.30 & 7.73 & 7.34 & 7.31 \\
\hline $\mathrm{CaO}$ & 2.61 & 2.54 & 2.51 & 2.30 & 2.33 & 2.28 & 2.20 & 2.07 & 1.97 & 1.90 \\
\hline $\mathrm{Na}_{2} \mathrm{O}$ & 2.49 & 2.54 & 2.53 & 2.51 & 2.52 & 2.61 & 2.56 & 2.53 & 2.45 & 2.46 \\
\hline $\mathrm{K}_{2} \mathrm{O}$ & 2.87 & 2.94 & 2.89 & 2.90 & 2.95 & 3.00 & 2.92 & 2.82 & 2.93 & 2.95 \\
\hline \multicolumn{11}{|l|}{ Std. Dev. } \\
\hline $\mathrm{SiO}_{2}$ & 8.14 & 8.15 & 8.18 & 8.00 & 8.03 & 8.04 & 7.78 & 8.13 & 8.18 & 8.26 \\
\hline $\mathrm{Al}_{2} \mathrm{O}_{3}$ & 7.02 & 7.07 & 7.09 & 7.02 & 7.07 & 7.08 & 6.49 & 6.61 & 6.71 & 6.82 \\
\hline $\mathrm{MgO}$ & 1.97 & 1.96 & 1.90 & 1.86 & 1.79 & 1.71 & 1.59 & 1.18 & 1.14 & 1.14 \\
\hline $\mathrm{FeO}$ & 5.23 & 5.25 & 5.66 & 5.76 & 5.80 & 5.71 & 5.79 & 5.73 & 5.51 & 5.60 \\
\hline $\mathrm{CaO}$ & 1.63 & 1.63 & 1.58 & 1.39 & 1.40 & 1.33 & 1.34 & 1.21 & 1.11 & 1.12 \\
\hline $\mathrm{Na}_{2} \mathrm{O}$ & 1.80 & 1.80 & 1.80 & 1.77 & 1.78 & 1.74 & 1.68 & 1.73 & 1.79 & 1.83 \\
\hline $\mathrm{K}_{2} \mathrm{O}$ & 1.33 & 1.31 & 1.34 & 1.38 & 1.38 & 1.38 & 1.37 & 1.37 & 1.29 & 1.30 \\
\hline$N$ & 268 & 264 & 264 & 238 & 225 & 207 & 187 & 167 & 157 & 151 \\
\hline
\end{tabular}


Table A.1: continued

\begin{tabular}{|c|c|c|c|c|c|c|c|c|c|c|}
\hline Depth $(\mathrm{km})$ & 5 & 10 & 15 & 20 & 25 & 30 & 35 & 40 & 45 & 50 \\
\hline $\mathrm{T}(\mathrm{C})$ & 90.90 & 154.56 & 210.78 & 262.50 & 311.49 & 358.82 & 405.15 & 450.86 & 496.21 & 541.33 \\
\hline $\mathrm{P}$ (kbar) & 1.62 & 3.23 & 4.85 & 6.47 & 8.09 & 9.70 & 11.32 & 12.94 & 14.55 & 16.17 \\
\hline \multicolumn{11}{|c|}{$V_{p}=6.4 \mathrm{~km} / \mathrm{s}$} \\
\hline \multicolumn{11}{|l|}{ Mean } \\
\hline $\mathrm{SiO}_{2}$ & 67.45 & 67.41 & 67.35 & 67.71 & 68.15 & 69.42 & 70.45 & 71.65 & 72.34 & 72.74 \\
\hline $\mathrm{Al}_{2} \mathrm{O}_{3}$ & 12.57 & 12.90 & 13.01 & 12.88 & 12.61 & 11.72 & 10.96 & 10.16 & 9.67 & 9.39 \\
\hline $\mathrm{MgO}$ & 3.85 & 3.67 & 3.63 & 3.60 & 3.51 & 3.35 & 3.25 & 3.15 & 2.99 & 3.02 \\
\hline $\mathrm{FeO}$ & 7.05 & 6.86 & 6.90 & 6.76 & 6.76 & 6.95 & 7.05 & 6.75 & 6.89 & 6.75 \\
\hline $\mathrm{CaO}$ & 3.16 & 3.19 & 3.07 & 2.96 & 2.90 & 2.69 & 2.59 & 2.57 & 2.52 & 2.45 \\
\hline $\mathrm{Na}_{2} \mathrm{O}$ & 3.29 & 3.35 & 3.40 & 3.40 & 3.35 & 3.16 & 2.99 & 2.86 & 2.74 & 2.72 \\
\hline $\mathrm{K}_{2} \mathrm{O}$ & 2.63 & 2.64 & 2.64 & 2.70 & 2.73 & 2.72 & 2.73 & 2.87 & 2.85 & 2.94 \\
\hline \multicolumn{11}{|l|}{ Std. Dev. } \\
\hline $\mathrm{SiO}_{2}$ & 9.60 & 9.68 & 9.79 & 9.74 & 9.48 & 9.58 & 9.61 & 9.56 & 9.21 & 9.17 \\
\hline $\mathrm{Al}_{2} \mathrm{O}_{3}$ & 8.62 & 8.48 & 8.57 & 8.56 & 8.41 & 8.26 & 8.20 & 8.12 & 7.96 & 7.78 \\
\hline $\mathrm{MgO}$ & 2.60 & 2.49 & 2.51 & 2.48 & 2.30 & 2.10 & 2.01 & 1.99 & 1.95 & 1.92 \\
\hline $\mathrm{FeO}$ & 5.20 & 5.19 & 5.12 & 5.16 & 5.30 & 5.13 & 5.07 & 5.02 & 5.14 & 5.21 \\
\hline $\mathrm{CaO}$ & 2.04 & 2.03 & 1.93 & 1.80 & 1.76 & 1.65 & 1.61 & 1.57 & 1.61 & 1.60 \\
\hline $\mathrm{Na}_{2} \mathrm{O}$ & 2.18 & 2.18 & 2.19 & 2.20 & 2.17 & 2.16 & 2.14 & 2.08 & 2.03 & 2.02 \\
\hline $\mathrm{K}_{2} \mathrm{O}$ & 1.15 & 1.17 & 1.16 & 1.18 & 1.22 & 1.21 & 1.20 & 1.27 & 1.31 & 1.30 \\
\hline$N$ & 290 & 286 & 278 & 269 & 257 & 265 & 291 & 302 & 297 & 274 \\
\hline \multicolumn{11}{|c|}{$V_{p}=6.5 \mathrm{~km} / \mathrm{s}$} \\
\hline Mean & & & & & & & & & & \\
\hline $\mathrm{SiO}_{2}$ & 60.87 & 61.37 & 61.66 & 62.31 & 62.90 & 63.75 & 64.64 & 65.36 & 65.58 & 65.69 \\
\hline $\mathrm{Al}_{2} \mathrm{O}_{3}$ & 16.64 & 16.31 & 16.16 & 15.87 & 15.46 & 14.86 & 14.27 & 13.79 & 13.59 & 13.70 \\
\hline $\mathrm{MgO}$ & 4.52 & 4.55 & 4.44 & 4.23 & 4.17 & 4.23 & 4.25 & 4.23 & 4.12 & 3.85 \\
\hline $\mathrm{FeO}$ & 7.76 & 7.76 & 7.80 & 7.73 & 7.78 & 7.76 & 7.65 & 7.46 & 7.65 & 7.62 \\
\hline $\mathrm{CaO}$ & 4.03 & 3.90 & 3.78 & 3.72 & 3.60 & 3.39 & 3.11 & 3.04 & 2.99 & 3.02 \\
\hline $\mathrm{Na}_{2} \mathrm{O}$ & 4.00 & 3.94 & 3.94 & 3.89 & 3.82 & 3.71 & 3.66 & 3.59 & 3.56 & 3.59 \\
\hline $\mathrm{K}_{2} \mathrm{O}$ & 2.18 & 2.19 & 2.23 & 2.27 & 2.29 & 2.32 & 2.43 & 2.54 & 2.52 & 2.54 \\
\hline \multicolumn{11}{|l|}{ Std. Dev. } \\
\hline $\mathrm{SiO}_{2}$ & 9.53 & 9.54 & 9.79 & 10.03 & 10.29 & 10.52 & 11.09 & 11.33 & 11.26 & 11.38 \\
\hline $\mathrm{Al}_{2} \mathrm{O}_{3}$ & 8.30 & 8.37 & 8.49 & 8.44 & 8.56 & 8.63 & 9.22 & 9.31 & 9.15 & 9.09 \\
\hline $\mathrm{MgO}$ & 2.96 & 2.93 & 2.81 & 2.79 & 2.77 & 2.74 & 2.72 & 2.71 & 2.75 & 2.63 \\
\hline $\mathrm{FeO}$ & 5.71 & 5.64 & 5.65 & 5.57 & 5.60 & 5.73 & 5.65 & 5.68 & 5.78 & 5.85 \\
\hline $\mathrm{CaO}$ & 2.75 & 2.65 & 2.55 & 2.44 & 2.45 & 2.31 & 2.00 & 2.00 & 1.96 & 1.98 \\
\hline $\mathrm{Na}_{2} \mathrm{O}$ & 2.17 & 2.20 & 2.23 & 2.22 & 2.25 & 2.25 & 2.34 & 2.35 & 2.33 & 2.31 \\
\hline $\mathrm{K}_{2} \mathrm{O}$ & 1.09 & 1.08 & 1.08 & 1.09 & 1.09 & 1.09 & 1.13 & 1.17 & 1.20 & 1.22 \\
\hline$N$ & 377 & 379 & 369 & 372 & 358 & 361 & 345 & 346 & 347 & 330 \\
\hline
\end{tabular}


Table A.1: continued

\begin{tabular}{|c|c|c|c|c|c|c|c|c|c|c|}
\hline Depth $(\mathrm{km})$ & 5 & 10 & 15 & 20 & 25 & 30 & 35 & 40 & 45 & 50 \\
\hline $\mathrm{T}(\mathrm{C})$ & 90.90 & 154.56 & 210.78 & 262.50 & 311.49 & 358.82 & 405.15 & 450.86 & 496.21 & 541.33 \\
\hline $\mathrm{P}$ (kbar) & 1.62 & 3.23 & 4.85 & 6.47 & 8.09 & 9.70 & 11.32 & 12.94 & 14.55 & 16.17 \\
\hline \multicolumn{11}{|c|}{$V_{p}=6.6 \mathrm{~km} / \mathrm{s}$} \\
\hline Mean & & & & & & & & & & \\
\hline $\mathrm{SiO}_{2}$ & 58.79 & 58.89 & 59.22 & 59.38 & 59.40 & 59.21 & 59.17 & 59.03 & 59.12 & 59.54 \\
\hline $\mathrm{Al}_{2} \mathrm{O}_{3}$ & 17.25 & 17.13 & 16.85 & 16.76 & 16.87 & 17.21 & 17.34 & 17.48 & 17.55 & 17.36 \\
\hline $\mathrm{MgO}$ & 5.48 & 5.49 & 5.54 & 5.49 & 5.26 & 5.12 & 5.11 & 5.02 & 4.92 & 4.66 \\
\hline $\mathrm{FeO}$ & 7.94 & 8.04 & 8.09 & 8.06 & 8.18 & 8.03 & 8.15 & 8.23 & 8.18 & 8.39 \\
\hline $\mathrm{CaO}$ & 4.77 & 4.62 & 4.51 & 4.47 & 4.43 & 4.37 & 4.12 & 4.02 & 3.90 & 3.77 \\
\hline $\mathrm{Na}_{2} \mathrm{O}$ & 3.91 & 3.93 & 3.89 & 3.89 & 3.92 & 4.04 & 4.11 & 4.18 & 4.24 & 4.21 \\
\hline $\mathrm{K}_{2} \mathrm{O}$ & 1.87 & 1.91 & 1.92 & 1.96 & 1.95 & 2.02 & 2.01 & 2.05 & 2.10 & 2.08 \\
\hline \multicolumn{11}{|l|}{ Std. Dev. } \\
\hline $\mathrm{SiO}_{2}$ & 9.74 & 10.02 & 10.11 & 10.16 & 10.48 & 10.33 & 10.44 & 10.54 & 10.59 & 10.91 \\
\hline $\mathrm{Al}_{2} \mathrm{O}_{3}$ & 8.32 & 8.56 & 8.56 & 8.52 & 8.73 & 8.57 & 8.64 & 8.67 & 8.64 & 8.87 \\
\hline $\mathrm{MgO}$ & 3.42 & 3.42 & 3.42 & 3.39 & 3.27 & 3.25 & 3.30 & 3.27 & 3.16 & 3.06 \\
\hline $\mathrm{FeO}$ & 5.61 & 5.63 & 5.70 & 5.63 & 5.66 & 5.80 & 5.85 & 5.80 & 5.86 & 5.90 \\
\hline $\mathrm{CaO}$ & 2.97 & 2.96 & 2.91 & 2.90 & 2.90 & 2.89 & 2.75 & 2.71 & 2.63 & 2.55 \\
\hline $\mathrm{Na}_{2} \mathrm{O}$ & 2.22 & 2.24 & 2.25 & 2.25 & 2.30 & 2.24 & 2.24 & 2.24 & 2.24 & 2.29 \\
\hline $\mathrm{K}_{2} \mathrm{O}$ & 1.08 & 1.07 & 1.08 & 1.07 & 1.06 & 1.08 & 1.10 & 1.10 & 1.11 & 1.12 \\
\hline$N$ & 516 & 514 & 493 & 487 & 478 & 477 & 456 & 447 & 438 & 429 \\
\hline \multicolumn{11}{|c|}{$V_{p}=6.7 \mathrm{~km} / \mathrm{s}$} \\
\hline Mean & & & & & & & & & & \\
\hline $\mathrm{SiO}_{2}$ & 56.64 & 56.49 & 56.52 & 56.77 & 56.87 & 56.86 & 56.83 & 56.89 & 56.90 & 57.69 \\
\hline $\mathrm{Al}_{2} \mathrm{O}_{3}$ & 17.71 & 17.83 & 17.88 & 17.74 & 17.82 & 17.91 & 18.14 & 18.24 & 18.26 & 17.75 \\
\hline $\mathrm{MgO}$ & 6.69 & 6.63 & 6.50 & 6.45 & 6.21 & 5.99 & 5.75 & 5.75 & 5.70 & 5.70 \\
\hline $\mathrm{FeO}$ & 8.29 & 8.34 & 8.42 & 8.43 & 8.43 & 8.48 & 8.52 & 8.51 & 8.60 & 8.41 \\
\hline $\mathrm{CaO}$ & 5.24 & 5.25 & 5.20 & 5.08 & 5.08 & 5.15 & 5.01 & 4.78 & 4.64 & 4.58 \\
\hline $\mathrm{Na}_{2} \mathrm{O}$ & 3.85 & 3.88 & 3.91 & 3.91 & 3.94 & 3.96 & 4.06 & 4.12 & 4.17 & 4.06 \\
\hline $\mathrm{K}_{2} \mathrm{O}$ & 1.59 & 1.59 & 1.59 & 1.63 & 1.65 & 1.66 & 1.70 & 1.73 & 1.75 & 1.80 \\
\hline \multicolumn{11}{|l|}{ Std. Dev. } \\
\hline $\mathrm{SiO}_{2}$ & 10.02 & 10.02 & 10.07 & 10.27 & 10.16 & 10.30 & 10.23 & 10.28 & 10.34 & 10.36 \\
\hline $\mathrm{Al}_{2} \mathrm{O}_{3}$ & 8.46 & 8.41 & 8.44 & 8.56 & 8.45 & 8.55 & 8.37 & 8.24 & 8.34 & 8.44 \\
\hline $\mathrm{MgO}$ & 3.57 & 3.61 & 3.62 & 3.63 & 3.48 & 3.40 & 3.40 & 3.37 & 3.37 & 3.35 \\
\hline $\mathrm{FeO}$ & 5.40 & 5.44 & 5.43 & 5.53 & 5.54 & 5.51 & 5.62 & 5.69 & 5.67 & 5.56 \\
\hline $\mathrm{CaO}$ & 3.35 & 3.35 & 3.33 & 3.20 & 3.18 & 3.21 & 3.13 & 2.95 & 2.89 & 2.87 \\
\hline $\mathrm{Na}_{2} \mathrm{O}$ & 2.33 & 2.32 & 2.30 & 2.31 & 2.30 & 2.34 & 2.28 & 2.22 & 2.23 & 2.24 \\
\hline $\mathrm{K}_{2} \mathrm{O}$ & 0.98 & 0.99 & 0.98 & 1.00 & 1.01 & 1.02 & 1.05 & 1.05 & 1.05 & 1.05 \\
\hline$N$ & 591 & 596 & 594 & 583 & 586 & 582 & 583 & 578 & 559 & 563 \\
\hline
\end{tabular}


Table A.1 continued

\begin{tabular}{|c|c|c|c|c|c|c|c|c|c|c|}
\hline Depth $(\mathrm{km})$ & 5 & 10 & 15 & 20 & 25 & 30 & 35 & 40 & 45 & 50 \\
\hline $\mathrm{T}(\mathrm{C})$ & 90.90 & 154.56 & 210.78 & 262.50 & 311.49 & 358.82 & 405.15 & 450.86 & 496.21 & 541.33 \\
\hline $\mathrm{P}(\mathrm{kbar})$ & 1.62 & 3.23 & 4.85 & 6.47 & 8.09 & 9.70 & 11.32 & 12.94 & 14.55 & 16.17 \\
\hline \multicolumn{11}{|c|}{$V_{p}=6.8 \mathrm{~km} / \mathrm{s}$} \\
\hline Mean & & & & & & & & & & \\
\hline $\mathrm{SiO}_{2}$ & 54.90 & 55.13 & 55.08 & 55.04 & 55.21 & 55.49 & 55.69 & 55.92 & 55.95 & 56.06 \\
\hline $\mathrm{Al}_{2} \mathrm{O}_{3}$ & 18.17 & 17.98 & 18.25 & 18.38 & 18.28 & 18.05 & 17.90 & 17.80 & 17.94 & 17.96 \\
\hline $\mathrm{MgO}$ & 7.99 & 8.00 & 7.73 & 7.51 & 7.59 & 7.58 & 7.48 & 7.34 & 7.24 & 7.03 \\
\hline $\mathrm{FeO}$ & 7.85 & 7.86 & 7.84 & 8.05 & 7.94 & 8.03 & 8.06 & 8.12 & 8.10 & 8.13 \\
\hline $\mathrm{CaO}$ & 5.84 & 5.83 & 5.82 & 5.71 & 5.60 & 5.54 & 5.56 & 5.52 & 5.39 & 5.35 \\
\hline $\mathrm{Na}_{2} \mathrm{O}$ & 3.80 & 3.76 & 3.82 & 3.87 & 3.88 & 3.83 & 3.81 & 3.79 & 3.85 & 3.89 \\
\hline $\mathrm{K}_{2} \mathrm{O}$ & 1.47 & 1.47 & 1.47 & 1.45 & 1.51 & 1.50 & 1.53 & 1.52 & 1.55 & 1.59 \\
\hline \multicolumn{11}{|l|}{ Std. Dev. } \\
\hline $\mathrm{SiO}_{2}$ & 9.79 & 9.72 & 9.80 & 9.89 & 9.86 & 10.01 & 10.09 & 10.30 & 10.24 & 10.19 \\
\hline $\mathrm{Al}_{2} \mathrm{O}_{3}$ & 8.32 & 8.26 & 8.27 & 8.36 & 8.33 & 8.43 & 8.45 & 8.53 & 8.46 & 8.42 \\
\hline $\mathrm{MgO}$ & 4.15 & 4.11 & 4.04 & 3.92 & 3.94 & 3.86 & 3.78 & 3.74 & 3.73 & 3.64 \\
\hline $\mathrm{FeO}$ & 5.28 & 5.24 & 5.27 & 5.33 & 5.33 & 5.27 & 5.26 & 5.31 & 5.26 & 5.20 \\
\hline $\mathrm{CaO}$ & 3.84 & 3.77 & 3.75 & 3.64 & 3.60 & 3.50 & 3.48 & 3.44 & 3.43 & 3.39 \\
\hline $\mathrm{Na}_{2} \mathrm{O}$ & 2.33 & 2.31 & 2.32 & 2.33 & 2.31 & 2.33 & 2.34 & 2.36 & 2.32 & 2.30 \\
\hline $\mathrm{K}_{2} \mathrm{O}$ & 0.91 & 0.90 & 0.91 & 0.90 & 0.93 & 0.93 & 0.95 & 0.94 & 0.94 & 0.95 \\
\hline$N$ & 674 & 684 & 685 & 674 & 683 & 683 & 683 & 668 & 661 & 650 \\
\hline \multicolumn{11}{|c|}{$V_{p}=6.9 \mathrm{~km} / \mathrm{s}$} \\
\hline Mean & & & & & & & & & & \\
\hline $\mathrm{SiO}_{2}$ & 54.21 & 54.32 & 54.59 & 54.48 & 54.59 & 54.78 & 55.11 & 55.31 & 55.64 & 55.35 \\
\hline $\mathrm{Al}_{2} \mathrm{O}_{3}$ & 17.82 & 17.74 & 17.55 & 17.77 & 17.68 & 17.64 & 17.44 & 17.36 & 17.25 & 17.53 \\
\hline $\mathrm{MgO}$ & 9.28 & 9.33 & 9.36 & 9.17 & 9.25 & 9.16 & 9.05 & 8.82 & 8.72 & 8.67 \\
\hline $\mathrm{FeO}$ & 7.06 & 7.11 & 7.03 & 7.09 & 7.17 & 7.10 & 7.06 & 7.17 & 7.09 & 7.23 \\
\hline $\mathrm{CaO}$ & 6.65 & 6.53 & 6.52 & 6.49 & 6.24 & 6.25 & 6.29 & 6.33 & 6.27 & 6.09 \\
\hline $\mathrm{Na}_{2} \mathrm{O}$ & 3.55 & 3.55 & 3.51 & 3.57 & 3.60 & 3.59 & 3.54 & 3.52 & 3.51 & 3.61 \\
\hline $\mathrm{K}_{2} \mathrm{O}$ & 1.43 & 1.44 & 1.45 & 1.45 & 1.48 & 1.49 & 1.51 & 1.50 & 1.53 & 1.52 \\
\hline \multicolumn{11}{|l|}{ Std. Dev. } \\
\hline $\mathrm{SiO}_{2}$ & 9.26 & 9.30 & 9.23 & 9.21 & 9.34 & 9.33 & 9.32 & 9.44 & 9.40 & 9.56 \\
\hline $\mathrm{Al}_{2} \mathrm{O}_{3}$ & 8.14 & 8.18 & 8.09 & 8.05 & 8.18 & 8.15 & 8.13 & 8.26 & 8.18 & 8.29 \\
\hline $\mathrm{MgO}$ & 4.90 & 4.90 & 4.85 & 4.79 & 4.81 & 4.75 & 4.66 & 4.61 & 4.57 & 4.51 \\
\hline $\mathrm{FeO}$ & 4.83 & 4.81 & 4.83 & 4.79 & 4.82 & 4.80 & 4.72 & 4.67 & 4.65 & 4.81 \\
\hline $\mathrm{CaO}$ & 4.29 & 4.17 & 4.15 & 4.15 & 4.09 & 4.02 & 3.93 & 3.96 & 3.80 & 3.71 \\
\hline $\mathrm{Na}_{2} \mathrm{O}$ & 2.19 & 2.19 & 2.18 & 2.18 & 2.20 & 2.18 & 2.18 & 2.23 & 2.21 & 2.24 \\
\hline $\mathrm{K}_{2} \mathrm{O}$ & 0.89 & 0.89 & 0.90 & 0.89 & 0.89 & 0.89 & 0.89 & 0.90 & 0.90 & 0.90 \\
\hline$N$ & 688 & 681 & 697 & 709 & 703 & 709 & 722 & 709 & 697 & 694 \\
\hline
\end{tabular}


Table A.1: continued

\begin{tabular}{|c|c|c|c|c|c|c|c|c|c|c|}
\hline Depth $(\mathrm{km})$ & 5 & 10 & 15 & 20 & 25 & 30 & 35 & 40 & 45 & 50 \\
\hline $\mathrm{T}(\mathrm{C})$ & 90.90 & 154.56 & 210.78 & 262.50 & 311.49 & 358.82 & 405.15 & 450.86 & 496.21 & 541.33 \\
\hline$P$ (kbar) & 1.62 & 3.23 & 4.85 & 6.47 & 8.09 & 9.70 & 11.32 & 12.94 & 14.55 & 16.17 \\
\hline \multicolumn{11}{|c|}{$V_{p}=7.0 \mathrm{~km} / \mathrm{s}$} \\
\hline Mean & & & & & & & & & & \\
\hline $\mathrm{SiO}_{2}$ & 52.93 & 52.85 & 53.09 & 53.37 & 53.45 & 53.50 & 53.60 & 53.77 & 54.13 & 54.25 \\
\hline $\mathrm{Al}_{2} \mathrm{O}_{3}$ & 17.57 & 17.64 & 17.41 & 17.27 & 17.29 & 17.34 & 17.41 & 17.38 & 17.21 & 17.32 \\
\hline $\mathrm{MgO}$ & 10.80 & 10.78 & 10.77 & 10.72 & 10.57 & 10.52 & 10.35 & 10.14 & 10.04 & 9.99 \\
\hline $\mathrm{FeO}$ & 7.00 & 7.05 & 7.08 & 6.97 & 7.01 & 6.96 & 6.91 & 7.03 & 7.00 & 6.98 \\
\hline $\mathrm{CaO}$ & 7.15 & 7.11 & 7.09 & 7.11 & 7.07 & 7.04 & 7.05 & 6.94 & 6.92 & 6.69 \\
\hline $\mathrm{Na}_{2} \mathrm{O}$ & 3.33 & 3.35 & 3.32 & 3.29 & 3.31 & 3.33 & 3.36 & 3.39 & 3.34 & 3.40 \\
\hline $\mathrm{K}_{2} \mathrm{O}$ & 1.23 & 1.22 & 1.25 & 1.28 & 1.30 & 1.32 & 1.34 & 1.37 & 1.37 & 1.38 \\
\hline \multicolumn{11}{|l|}{ Std. Dev. } \\
\hline $\mathrm{SiO}_{2}$ & 8.92 & 8.94 & 8.92 & 8.88 & 8.93 & 8.90 & 8.97 & 8.98 & 9.11 & 9.24 \\
\hline $\mathrm{Al}_{2} \mathrm{O}_{3}$ & 7.81 & 7.86 & 7.86 & 7.80 & 7.82 & 7.79 & 7.77 & 7.81 & 7.96 & 8.00 \\
\hline $\mathrm{MgO}$ & 5.40 & 5.45 & 5.44 & 5.45 & 5.52 & 5.54 & 5.53 & 5.42 & 5.31 & 5.26 \\
\hline $\mathrm{FeO}$ & 4.76 & 4.73 & 4.70 & 4.68 & 4.69 & 4.67 & 4.58 & 4.62 & 4.56 & 4.65 \\
\hline $\mathrm{CaO}$ & 4.71 & 4.69 & 4.62 & 4.61 & 4.61 & 4.61 & 4.54 & 4.40 & 4.28 & 4.14 \\
\hline $\mathrm{Na}_{2} \mathrm{O}$ & 2.09 & 2.08 & 2.07 & 2.04 & 2.04 & 2.04 & 2.04 & 2.03 & 2.07 & 2.07 \\
\hline $\mathrm{K}_{2} \mathrm{O}$ & 0.83 & 0.83 & 0.84 & 0.86 & 0.86 & 0.86 & 0.85 & 0.87 & 0.88 & 0.89 \\
\hline$N$ & 613 & 607 & 632 & 653 & 649 & 663 & 664 & 663 & 674 & 688 \\
\hline \multicolumn{11}{|c|}{$V_{p}=7.1 \mathrm{~km} / \mathrm{s}$} \\
\hline Mean & & & & & & & & & & \\
\hline $\mathrm{SiO}_{2}$ & 51.30 & 51.34 & 51.28 & 51.50 & 51.59 & 51.72 & 51.65 & 51.90 & 51.88 & 52.16 \\
\hline $\mathrm{Al}_{2} \mathrm{O}_{3}$ & 17.64 & 17.59 & 17.76 & 17.65 & 17.70 & 17.61 & 17.74 & 17.76 & 17.93 & 17.80 \\
\hline $\mathrm{MgO}$ & 12.31 & 12.24 & 12.12 & 12.04 & 11.96 & 12.03 & 11.82 & 11.62 & 11.29 & 11.11 \\
\hline $\mathrm{FeO}$ & 7.16 & 7.15 & 7.11 & 7.05 & 6.98 & 7.04 & 7.23 & 7.09 & 7.21 & 7.21 \\
\hline $\mathrm{CaO}$ & 7.23 & 7.34 & 7.37 & 7.40 & 7.42 & 7.21 & 7.12 & 7.16 & 7.22 & 7.28 \\
\hline $\mathrm{Na}_{2} \mathrm{O}$ & 3.29 & 3.26 & 3.29 & 3.27 & 3.27 & 3.28 & 3.34 & 3.34 & 3.37 & 3.33 \\
\hline $\mathrm{K}_{2} \mathrm{O}$ & 1.08 & 1.08 & 1.08 & 1.10 & 1.10 & 1.11 & 1.11 & 1.13 & 1.12 & 1.12 \\
\hline \multicolumn{11}{|l|}{ Std. Dev. } \\
\hline $\mathrm{SiO}_{2}$ & 8.71 & 8.65 & 8.73 & 8.82 & 8.78 & 8.84 & 9.08 & 9.13 & 9.24 & 9.22 \\
\hline $\mathrm{Al}_{2} \mathrm{O}_{3}$ & 7.70 & 7.69 & 7.72 & 7.74 & 7.70 & 7.73 & 7.83 & 7.85 & 7.94 & 7.88 \\
\hline $\mathrm{MgO}$ & 5.88 & 5.93 & 5.93 & 5.90 & 5.83 & 5.80 & 5.77 & 5.67 & 5.61 & 5.56 \\
\hline $\mathrm{FeO}$ & 5.02 & 4.98 & 4.98 & 5.00 & 4.96 & 4.97 & 4.97 & 4.96 & 4.98 & 4.97 \\
\hline $\mathrm{CaO}$ & 5.01 & 5.05 & 5.06 & 5.06 & 4.96 & 4.89 & 4.92 & 4.79 & 4.79 & 4.82 \\
\hline $\mathrm{Na}_{2} \mathrm{O}$ & 2.06 & 2.06 & 2.07 & 2.06 & 2.03 & 2.03 & 2.05 & 2.04 & 2.07 & 2.06 \\
\hline $\mathrm{K}_{2} \mathrm{O}$ & 0.79 & 0.78 & 0.79 & 0.79 & 0.78 & 0.78 & 0.80 & 0.78 & 0.79 & 0.79 \\
\hline$N$ & 550 & 567 & 566 & 575 & 588 & 598 & 588 & 612 & 636 & 652 \\
\hline
\end{tabular}


Table A.1: continued

\begin{tabular}{|c|c|c|c|c|c|c|c|c|c|c|}
\hline Depth $(\mathrm{km})$ & 5 & 10 & 15 & 20 & 25 & 30 & 35 & 40 & 45 & 50 \\
\hline $\mathrm{T}(\mathrm{C})$ & 90.90 & 154.56 & 210.78 & 262.50 & 311.49 & 358.82 & 405.15 & 450.86 & 496.21 & 541.33 \\
\hline $\mathrm{P}$ (kbar) & 1.62 & 3.23 & 4.85 & 6.47 & 8.09 & 9.70 & 11.32 & 12.94 & 14.55 & 16.17 \\
\hline \multicolumn{11}{|c|}{$V_{p}=7.2 \mathrm{~km} / \mathrm{s}$} \\
\hline Mean & & & & & & & & & & \\
\hline $\mathrm{SiO}_{2}$ & 50.37 & 50.47 & 50.24 & 50.30 & 50.52 & 50.58 & 50.81 & 51.06 & 50.92 & 51.15 \\
\hline $\mathrm{Al}_{2} \mathrm{O}_{3}$ & 17.65 & 17.65 & 17.88 & 17.89 & 17.75 & 17.74 & 17.66 & 17.59 & 17.61 & 17.43 \\
\hline $\mathrm{MgO}$ & 12.81 & 12.72 & 12.68 & 12.64 & 12.63 & 12.66 & 12.61 & 12.62 & 12.55 & 12.57 \\
\hline $\mathrm{FeO}$ & 7.24 & 7.14 & 7.10 & 7.06 & 7.04 & 7.01 & 6.96 & 6.86 & 6.99 & 7.00 \\
\hline $\mathrm{CaO}$ & 7.69 & 7.80 & 7.80 & 7.82 & 7.84 & 7.79 & 7.78 & 7.70 & 7.73 & 7.66 \\
\hline $\mathrm{Na}_{2} \mathrm{O}$ & 3.23 & 3.21 & 3.27 & 3.26 & 3.21 & 3.21 & 3.18 & 3.16 & 3.18 & 3.16 \\
\hline $\mathrm{K}_{2} \mathrm{O}$ & 1.03 & 1.03 & 1.04 & 1.04 & 1.02 & 1.02 & 1.02 & 1.02 & 1.03 & 1.05 \\
\hline \multicolumn{11}{|l|}{ Std. Dev. } \\
\hline $\mathrm{SiO}_{2}$ & 8.51 & 8.53 & 8.64 & 8.64 & 8.55 & 8.63 & 8.54 & 8.47 & 8.39 & 8.51 \\
\hline $\mathrm{Al}_{2} \mathrm{O}_{3}$ & 7.86 & 7.85 & 7.93 & 7.87 & 7.75 & 7.79 & 7.75 & 7.62 & 7.53 & 7.57 \\
\hline $\mathrm{MgO}$ & 6.35 & 6.32 & 6.29 & 6.24 & 6.13 & 6.10 & 6.10 & 6.10 & 6.14 & 6.06 \\
\hline $\mathrm{FeO}$ & 4.85 & 4.88 & 4.91 & 4.88 & 4.82 & 4.85 & 4.93 & 4.88 & 5.01 & 4.97 \\
\hline $\mathrm{CaO}$ & 5.14 & 5.19 & 5.19 & 5.20 & 5.12 & 5.08 & 5.09 & 5.11 & 5.14 & 5.01 \\
\hline $\mathrm{Na}_{2} \mathrm{O}$ & 2.00 & 2.01 & 2.02 & 2.01 & 1.99 & 2.00 & 1.98 & 1.98 & 1.95 & 1.95 \\
\hline $\mathrm{K}_{2} \mathrm{O}$ & 0.75 & 0.76 & 0.75 & 0.75 & 0.74 & 0.75 & 0.75 & 0.74 & 0.73 & 0.73 \\
\hline$N$ & 471 & 481 & 481 & 488 & 506 & 499 & 524 & 555 & 568 & 583 \\
\hline \multicolumn{11}{|c|}{$V_{p}=7.3 \mathrm{~km} / \mathrm{s}$} \\
\hline Mean & & & & & & & & & & \\
\hline $\mathrm{SiO}_{2}$ & 48.51 & 48.46 & 48.53 & 48.83 & 48.98 & 49.00 & 49.50 & 49.59 & 49.71 & 49.54 \\
\hline $\mathrm{Al}_{2} \mathrm{O}_{3}$ & 18.91 & 19.06 & 18.89 & 18.70 & 18.62 & 18.64 & 18.28 & 18.07 & 17.97 & 18.22 \\
\hline $\mathrm{MgO}$ & 12.70 & 12.60 & 12.71 & 12.71 & 12.63 & 12.53 & 12.69 & 12.83 & 12.98 & 12.94 \\
\hline $\mathrm{FeO}$ & 6.66 & 6.58 & 6.62 & 6.58 & 6.65 & 6.64 & 6.44 & 6.54 & 6.51 & 6.54 \\
\hline $\mathrm{CaO}$ & 9.03 & 9.09 & 9.08 & 9.05 & 8.98 & 9.09 & 9.07 & 8.99 & 8.87 & 8.67 \\
\hline $\mathrm{Na}_{2} \mathrm{O}$ & 3.28 & 3.30 & 3.26 & 3.22 & 3.21 & 3.19 & 3.10 & 3.06 & 3.05 & 3.15 \\
\hline $\mathrm{K}_{2} \mathrm{O}$ & 0.93 & 0.92 & 0.92 & 0.93 & 0.92 & 0.91 & 0.93 & 0.92 & 0.93 & 0.95 \\
\hline \multicolumn{11}{|l|}{ Std. Dev. } \\
\hline $\mathrm{SiO}_{2}$ & 7.84 & 7.85 & 7.82 & 8.00 & 8.01 & 8.02 & 8.07 & 8.06 & 8.04 & 8.13 \\
\hline $\mathrm{Al}_{2} \mathrm{O}_{3}$ & 7.70 & 7.64 & 7.67 & 7.74 & 7.75 & 7.74 & 7.71 & 7.69 & 7.65 & 7.67 \\
\hline $\mathrm{MgO}$ & 6.86 & 6.88 & 6.84 & 6.72 & 6.78 & 6.74 & 6.69 & 6.74 & 6.74 & 6.84 \\
\hline $\mathrm{FeO}$ & 4.29 & 4.32 & 4.31 & 4.30 & 4.35 & 4.31 & 4.37 & 4.44 & 4.48 & 4.42 \\
\hline $\mathrm{CaO}$ & 5.57 & 5.59 & 5.53 & 5.47 & 5.39 & 5.46 & 5.40 & 5.48 & 5.50 & 5.51 \\
\hline $\mathrm{Na}_{2} \mathrm{O}$ & 1.87 & 1.87 & 1.84 & 1.86 & 1.88 & 1.88 & 1.87 & 1.85 & 1.83 & 1.81 \\
\hline $\mathrm{K}_{2} \mathrm{O}$ & 0.72 & 0.71 & 0.70 & 0.71 & 0.72 & 0.72 & 0.70 & 0.70 & 0.69 & 0.70 \\
\hline$N$ & 413 & 406 & 413 & 421 & 427 & 434 & 462 & 471 & 484 & 471 \\
\hline
\end{tabular}


Table A.1: $\quad$ continued

\begin{tabular}{|c|c|c|c|c|c|c|c|c|c|c|}
\hline Depth $(\mathrm{km})$ & 5 & 10 & 15 & 20 & 25 & 30 & 35 & 40 & 45 & 50 \\
\hline $\mathrm{T}(\mathrm{C})$ & 90.90 & 154.56 & 210.78 & 262.50 & 311.49 & 358.82 & 405.15 & 450.86 & 496.21 & 541.33 \\
\hline $\mathrm{P}(\mathrm{kbar})$ & 1.62 & 3.23 & 4.85 & 6.47 & 8.09 & 9.70 & 11.32 & 12.94 & 14.55 & 16.17 \\
\hline \multicolumn{11}{|c|}{$V_{p}=7.4 \mathrm{~km} / \mathrm{s}$} \\
\hline \multicolumn{11}{|l|}{ Mean } \\
\hline $\mathrm{SiO}_{2}$ & 47.43 & 47.45 & 47.78 & 47.86 & 47.80 & 47.82 & 47.72 & 47.74 & 47.88 & 48.04 \\
\hline $\mathrm{Al}_{2} \mathrm{O}_{3}$ & 19.05 & 19.04 & 18.78 & 18.74 & 18.84 & 18.96 & 19.02 & 19.13 & 19.11 & 19.05 \\
\hline $\mathrm{MgO}$ & 13.91 & 13.92 & 13.92 & 13.92 & 13.86 & 13.48 & 13.48 & 13.31 & 13.25 & 13.04 \\
\hline $\mathrm{FeO}$ & 6.05 & 6.03 & 5.99 & 5.99 & 6.01 & 6.07 & 6.12 & 6.02 & 6.11 & 6.05 \\
\hline $\mathrm{CaO}$ & 9.57 & 9.56 & 9.64 & 9.60 & 9.58 & 9.72 & 9.68 & 9.81 & 9.63 & 9.85 \\
\hline $\mathrm{Na}_{2} \mathrm{O}$ & 3.16 & 3.16 & 3.08 & 3.07 & 3.10 & 3.12 & 3.14 & 3.15 & 3.18 & 3.13 \\
\hline $\mathrm{K}_{2} \mathrm{O}$ & 0.84 & 0.84 & 0.83 & 0.84 & 0.83 & 0.84 & 0.84 & 0.85 & 0.85 & 0.85 \\
\hline \multicolumn{11}{|l|}{ Std. Dev. } \\
\hline $\mathrm{SiO}_{2}$ & 7.30 & 7.28 & 7.23 & 7.23 & 7.27 & 7.29 & 7.31 & 7.30 & 7.48 & 7.44 \\
\hline $\mathrm{Al}_{2} \mathrm{O}_{3}$ & 7.40 & 7.35 & 7.25 & 7.25 & 7.30 & 7.26 & 7.29 & 7.20 & 7.27 & 7.20 \\
\hline $\mathrm{MgO}$ & 7.50 & 7.51 & 7.47 & 7.48 & 7.49 & 7.47 & 7.44 & 7.42 & 7.40 & 7.45 \\
\hline $\mathrm{FeO}$ & 4.16 & 4.14 & 4.10 & 4.10 & 4.09 & 4.09 & 4.15 & 4.13 & 4.15 & 4.12 \\
\hline $\mathrm{CaO}$ & 5.90 & 5.87 & 5.87 & 5.86 & 5.85 & 5.91 & 5.91 & 5.89 & 5.84 & 5.92 \\
\hline $\mathrm{Na}_{2} \mathrm{O}$ & 1.82 & 1.82 & 1.80 & 1.81 & 1.80 & 1.79 & 1.81 & 1.79 & 1.80 & 1.80 \\
\hline $\mathrm{K}_{2} \mathrm{O}$ & 0.68 & 0.68 & 0.69 & 0.69 & 0.69 & 0.70 & 0.70 & 0.70 & 0.71 & 0.70 \\
\hline$N$ & 344 & 345 & 354 & 361 & 361 & 365 & 364 & 370 & 367 & 378 \\
\hline \multicolumn{11}{|c|}{$V_{p}=7.5 \mathrm{~km} / \mathrm{s}$} \\
\hline Mean & & & & & & & & & & \\
\hline $\mathrm{SiO}_{2}$ & 47.57 & 47.36 & 47.36 & 47.31 & 47.37 & 47.42 & 47.41 & 47.56 & 47.63 & 47.83 \\
\hline $\mathrm{Al}_{2} \mathrm{O}_{3}$ & 18.18 & 18.28 & 18.32 & 18.35 & 18.41 & 18.37 & 18.42 & 18.44 & 18.45 & 18.11 \\
\hline $\mathrm{MgO}$ & 14.98 & 15.01 & 14.99 & 15.03 & 14.73 & 14.70 & 14.70 & 14.61 & 14.50 & 14.52 \\
\hline $\mathrm{FeO}$ & 5.83 & 5.79 & 5.80 & 5.81 & 5.76 & 5.79 & 5.74 & 5.69 & 5.71 & 5.76 \\
\hline $\mathrm{CaO}$ & 9.72 & 9.84 & 9.76 & 9.74 & 10.00 & 9.98 & 9.97 & 9.93 & 9.92 & 10.12 \\
\hline $\mathrm{Na}_{2} \mathrm{O}$ & 2.92 & 2.92 & 2.95 & 2.95 & 2.93 & 2.93 & 2.94 & 2.95 & 2.96 & 2.84 \\
\hline $\mathrm{K}_{2} \mathrm{O}$ & 0.82 & 0.80 & 0.82 & 0.81 & 0.81 & 0.82 & 0.82 & 0.83 & 0.84 & 0.81 \\
\hline \multicolumn{11}{|l|}{ Std. Dev. } \\
\hline $\mathrm{SiO}_{2}$ & 7.22 & 7.23 & 7.23 & 7.20 & 7.15 & 7.08 & 7.01 & 7.04 & 7.13 & 7.07 \\
\hline $\mathrm{Al}_{2} \mathrm{O}_{3}$ & 6.65 & 6.53 & 6.61 & 6.56 & 6.45 & 6.38 & 6.36 & 6.33 & 6.28 & 6.40 \\
\hline $\mathrm{MgO}$ & 8.00 & 7.87 & 7.92 & 7.97 & 7.96 & 8.04 & 8.05 & 8.04 & 8.04 & 7.83 \\
\hline $\mathrm{FeO}$ & 3.98 & 3.92 & 3.93 & 3.91 & 3.85 & 3.94 & 3.92 & 3.90 & 3.89 & 3.93 \\
\hline $\mathrm{CaO}$ & 6.27 & 6.17 & 6.24 & 6.23 & 6.30 & 6.28 & 6.27 & 6.23 & 6.20 & 6.05 \\
\hline $\mathrm{Na}_{2} \mathrm{O}$ & 1.81 & 1.78 & 1.82 & 1.80 & 1.78 & 1.76 & 1.75 & 1.75 & 1.74 & 1.75 \\
\hline $\mathrm{K}_{2} \mathrm{O}$ & 0.64 & 0.64 & 0.66 & 0.66 & 0.67 & 0.67 & 0.66 & 0.67 & 0.70 & 0.66 \\
\hline$N$ & 246 & 258 & 256 & 263 & 274 & 273 & 275 & 282 & 289 & 312 \\
\hline
\end{tabular}


Table A.1: continued

\begin{tabular}{|c|c|c|c|c|c|c|c|c|c|c|}
\hline Depth $(\mathrm{km})$ & 5 & 10 & 15 & 20 & 25 & 30 & 35 & 40 & 45 & 50 \\
\hline $\mathrm{T}(\mathrm{C})$ & 90.90 & 154.56 & 210.78 & 262.50 & 311.49 & 358.82 & 405.15 & 450.86 & 496.21 & 541.33 \\
\hline P (kbar) & 1.62 & 3.23 & 4.85 & 6.47 & 8.09 & 9.70 & 11.32 & 12.94 & 14.55 & 16.17 \\
\hline \multicolumn{11}{|c|}{$V_{p}=7.6 \mathrm{~km} / \mathrm{s}$} \\
\hline Mean & & & & & & & & & & \\
\hline $\mathrm{SiO}_{2}$ & 44.87 & 44.85 & 44.89 & 44.82 & 44.79 & 45.03 & 45.05 & 44.95 & 44.44 & 44.52 \\
\hline $\mathrm{Al}_{2} \mathrm{O}_{3}$ & 20.49 & 20.44 & 20.30 & 20.30 & 20.35 & 20.05 & 20.08 & 20.14 & 20.49 & 20.50 \\
\hline $\mathrm{MgO}$ & 14.13 & 14.18 & 14.27 & 14.27 & 14.26 & 14.58 & 14.62 & 14.62 & 14.55 & 14.42 \\
\hline $\mathrm{FeO}$ & 4.87 & 4.92 & 5.15 & 5.19 & 5.12 & 5.09 & 4.97 & 5.02 & 5.19 & 5.20 \\
\hline $\mathrm{CaO}$ & 11.44 & 11.40 & 11.22 & 11.26 & 11.33 & 11.19 & 11.22 & 11.18 & 11.23 & 11.24 \\
\hline $\mathrm{Na}_{2} \mathrm{O}$ & 3.29 & 3.28 & 3.28 & 3.27 & 3.27 & 3.20 & 3.19 & 3.22 & 3.28 & 3.29 \\
\hline $\mathrm{K}_{2} \mathrm{O}$ & 0.93 & 0.93 & 0.91 & 0.90 & 0.90 & 0.88 & 0.88 & 0.88 & 0.83 & 0.84 \\
\hline \multicolumn{11}{|l|}{ Std. Dev. } \\
\hline $\mathrm{SiO}_{2}$ & 7.31 & 7.27 & 7.35 & 7.32 & 7.46 & 7.49 & 7.52 & 7.64 & 8.08 & 8.13 \\
\hline $\mathrm{Al}_{2} \mathrm{O}_{3}$ & 6.01 & 6.00 & 6.34 & 6.40 & 6.47 & 6.53 & 6.50 & 6.72 & 6.93 & 6.92 \\
\hline $\mathrm{MgO}$ & 7.65 & 7.65 & 7.66 & 7.65 & 7.62 & 7.62 & 7.62 & 7.56 & 7.35 & 7.30 \\
\hline $\mathrm{FeO}$ & 3.61 & 3.64 & 3.80 & 3.81 & 3.80 & 3.77 & 3.57 & 3.60 & 3.59 & 3.61 \\
\hline $\mathrm{CaO}$ & 6.02 & 6.02 & 6.00 & 6.00 & 5.96 & 5.91 & 5.84 & 5.83 & 5.72 & 5.73 \\
\hline $\mathrm{Na}_{2} \mathrm{O}$ & 1.70 & 1.69 & 1.75 & 1.74 & 1.77 & 1.76 & 1.73 & 1.76 & 1.81 & 1.81 \\
\hline $\mathrm{K}_{2} \mathrm{O}$ & 0.73 & 0.73 & 0.74 & 0.71 & 0.70 & 0.67 & 0.66 & 0.66 & 0.61 & 0.61 \\
\hline$N$ & 215 & 217 & 217 & 219 & 223 & 231 & 234 & 241 & 265 & 270 \\
\hline
\end{tabular}


Table A.2: Mean and standard deviation of major element oxides as a function of $V_{p}$ for a cold continental geotherm $\left(q_{s}=35 \mathrm{mWm}^{-2}\right) . N$ denotes number of synthetic compositions in each $V_{p}$ bin.

\begin{tabular}{|c|c|c|c|c|c|c|c|c|c|c|}
\hline Depth $(\mathrm{km})$ & 5 & 10 & 15 & 20 & 25 & 30 & 35 & 40 & 45 & 50 \\
\hline $\mathrm{T}(\mathrm{C})$ & 61.99 & 101.36 & 136.10 & 168.04 & 198.27 & 227.47 & 256.05 & 284.25 & 312.21 & 340.04 \\
\hline $\mathrm{P}$ (kbar) & 1.62 & 3.23 & 4.85 & 6.47 & 8.09 & 9.70 & 11.32 & 12.94 & 14.55 & 16.17 \\
\hline \multicolumn{11}{|c|}{$V_{p}=6.2 \mathrm{~km} / \mathrm{s}$} \\
\hline Mean & & & & & & & & & & \\
\hline $\mathrm{SiO}_{2}$ & 76.34 & 76.79 & 77.40 & 77.49 & 76.25 & 77.05 & 79.84 & 80.77 & 83.28 & 81.13 \\
\hline $\mathrm{Al}_{2} \mathrm{O}_{3}$ & 7.11 & 6.89 & 6.39 & 6.03 & 7.20 & 6.91 & 8.24 & 7.36 & 5.44 & 7.45 \\
\hline $\mathrm{MgO}$ & 2.10 & 2.07 & 2.07 & 1.60 & 1.25 & 0.94 & 0.75 & 0.69 & 0.99 & 1.10 \\
\hline $\mathrm{FeO}$ & 7.32 & 7.26 & 7.29 & 7.79 & 9.35 & 9.11 & 4.97 & 5.34 & 5.54 & 4.73 \\
\hline $\mathrm{CaO}$ & 2.00 & 1.89 & 1.84 & 2.01 & 1.44 & 1.51 & 1.20 & 1.23 & 0.76 & 0.69 \\
\hline $\mathrm{Na}_{2} \mathrm{O}$ & 2.22 & 2.18 & 2.07 & 2.07 & 2.13 & 2.05 & 2.20 & 1.93 & 1.44 & 2.06 \\
\hline $\mathrm{K}_{2} \mathrm{O}$ & 2.92 & 2.93 & 2.94 & 3.02 & 2.39 & 2.43 & 2.82 & 2.69 & 2.57 & 2.85 \\
\hline \multicolumn{11}{|l|}{ Std. Dev. } \\
\hline $\mathrm{SiO}_{2}$ & 6.52 & 6.19 & 5.77 & 6.09 & 6.68 & 7.67 & 6.98 & 6.54 & 6.32 & 7.11 \\
\hline $\mathrm{Al}_{2} \mathrm{O}_{3}$ & 5.28 & 5.08 & 4.79 & 4.72 & 5.42 & 5.59 & 6.22 & 5.75 & 5.93 & 6.66 \\
\hline $\mathrm{MgO}$ & 1.11 & 1.13 & 1.15 & 0.91 & 0.79 & 0.54 & 0.55 & 0.54 & 0.34 & 0.38 \\
\hline $\mathrm{FeO}$ & 5.49 & 5.44 & 5.46 & 6.16 & 6.72 & 6.92 & 2.24 & 2.00 & 2.39 & 2.68 \\
\hline $\mathrm{CaO}$ & 1.14 & 1.16 & 1.15 & 1.17 & 0.85 & 0.84 & 0.75 & 0.77 & 0.20 & 0.23 \\
\hline $\mathrm{Na}_{2} \mathrm{O}$ & 1.40 & 1.36 & 1.32 & 1.25 & 1.54 & 1.56 & 1.85 & 1.69 & 1.82 & 2.05 \\
\hline $\mathrm{K}_{2} \mathrm{O}$ & 1.31 & 1.31 & 1.30 & 1.47 & 1.13 & 0.97 & 0.79 & 0.71 & 0.83 & 0.93 \\
\hline$N$ & 147 & 141 & 131 & 85 & 49 & 32 & 22 & 20 & 14 & 9 \\
\hline \multicolumn{11}{|c|}{$V_{p}=6.3 \mathrm{~km} / \mathrm{s}$} \\
\hline Mean & & & & & & & & & & \\
\hline $\mathrm{SiO}_{2}$ & 73.59 & 73.79 & 74.24 & 74.11 & 74.12 & 75.47 & 76.07 & 76.28 & 77.47 & 76.25 \\
\hline $\mathrm{Al}_{2} \mathrm{O}_{3}$ & 8.67 & 8.37 & 8.25 & 8.45 & 8.55 & 7.83 & 7.41 & 7.11 & 6.01 & 7.20 \\
\hline $\mathrm{MgO}$ & 2.87 & 2.89 & 2.72 & 2.50 & 2.05 & 2.05 & 2.03 & 1.89 & 1.57 & 1.25 \\
\hline $\mathrm{FeO}$ & 6.93 & 7.25 & 7.02 & 7.20 & 7.79 & 7.34 & 7.36 & 7.61 & 7.95 & 9.35 \\
\hline $\mathrm{CaO}$ & 2.53 & 2.30 & 2.32 & 2.18 & 2.10 & 1.98 & 1.87 & 1.90 & 1.97 & 1.44 \\
\hline $\mathrm{Na}_{2} \mathrm{O}$ & 2.53 & 2.51 & 2.49 & 2.58 & 2.57 & 2.42 & 2.33 & 2.28 & 2.06 & 2.13 \\
\hline $\mathrm{K}_{2} \mathrm{O}$ & 2.90 & 2.90 & 2.96 & 2.98 & 2.83 & 2.93 & 2.94 & 2.94 & 2.99 & 2.39 \\
\hline \multicolumn{11}{|l|}{ Std. Dev. } \\
\hline $\mathrm{SiO}_{2}$ & 8.14 & 8.01 & 8.08 & 7.64 & 7.96 & 8.23 & 7.73 & 8.00 & 6.24 & 6.68 \\
\hline $\mathrm{Al}_{2} \mathrm{O}_{3}$ & 7.04 & 7.02 & 7.04 & 6.44 & 6.58 & 6.74 & 6.33 & 6.45 & 4.83 & 5.42 \\
\hline $\mathrm{MgO}$ & 1.94 & 1.86 & 1.73 & 1.67 & 1.18 & 1.14 & 1.14 & 1.10 & 0.92 & 0.79 \\
\hline $\mathrm{FeO}$ & 5.64 & 5.76 & 5.73 & 5.82 & 5.81 & 5.42 & 5.53 & 5.64 & 6.24 & 6.72 \\
\hline $\mathrm{CaO}$ & 1.61 & 1.39 & 1.41 & 1.33 & 1.21 & 1.13 & 1.13 & 1.13 & 1.18 & 0.85 \\
\hline $\mathrm{Na}_{2} \mathrm{O}$ & 1.79 & 1.77 & 1.77 & 1.65 & 1.71 & 1.80 & 1.70 & 1.74 & 1.27 & 1.54 \\
\hline $\mathrm{K}_{2} \mathrm{O}$ & 1.33 & 1.39 & 1.38 & 1.39 & 1.39 & 1.30 & 1.31 & 1.36 & 1.45 & 1.13 \\
\hline$N$ & 269 & 237 & 214 & 190 & 162 & 152 & 144 & 124 & 81 & 49 \\
\hline
\end{tabular}


Table A.2: continued

\begin{tabular}{|c|c|c|c|c|c|c|c|c|c|c|}
\hline Depth $(\mathrm{km})$ & 5 & 10 & 15 & 20 & 25 & 30 & 35 & 40 & 45 & 50 \\
\hline $\mathrm{T}(\mathrm{C})$ & 61.99 & 101.36 & 136.10 & 168.04 & 198.27 & 227.47 & 256.05 & 284.25 & 312.21 & 340.04 \\
\hline $\mathrm{P}(\mathrm{kbar})$ & 1.62 & 3.23 & 4.85 & 6.47 & 8.09 & 9.70 & 11.32 & 12.94 & 14.55 & 16.17 \\
\hline \multicolumn{11}{|c|}{$V_{p}=6.4 \mathrm{~km} / \mathrm{s}$} \\
\hline Mean & & & & & & & & & & \\
\hline $\mathrm{SiO}_{2}$ & 67.43 & 67.99 & 68.48 & 70.21 & 72.03 & 72.77 & 73.51 & 73.60 & 73.90 & 74.42 \\
\hline $\mathrm{Al}_{2} \mathrm{O}_{3}$ & 12.92 & 12.71 & 12.36 & 11.19 & 9.93 & 9.46 & 8.73 & 8.79 & 8.66 & 8.38 \\
\hline $\mathrm{MgO}$ & 3.67 & 3.59 & 3.33 & 3.29 & 3.10 & 2.97 & 2.83 & 2.68 & 2.42 & 2.03 \\
\hline $\mathrm{FeO}$ & 6.79 & 6.70 & 6.98 & 6.98 & 6.67 & 6.69 & 7.04 & 7.01 & 7.27 & 7.79 \\
\hline $\mathrm{CaO}$ & 3.19 & 2.96 & 2.91 & 2.59 & 2.57 & 2.48 & 2.41 & 2.30 & 2.22 & 2.06 \\
\hline $\mathrm{Na}_{2} \mathrm{O}$ & 3.36 & 3.35 & 3.27 & 3.04 & 2.81 & 2.72 & 2.58 & 2.65 & 2.61 & 2.52 \\
\hline $\mathrm{K}_{2} \mathrm{O}$ & 2.65 & 2.71 & 2.68 & 2.72 & 2.90 & 2.93 & 2.92 & 3.00 & 2.94 & 2.82 \\
\hline \multicolumn{11}{|l|}{ Std. Dev. } \\
\hline $\mathrm{SiO}_{2}$ & 9.71 & 9.47 & 9.51 & 9.58 & 9.43 & 9.19 & 8.76 & 8.95 & 7.78 & 8.06 \\
\hline $\mathrm{Al}_{2} \mathrm{O}_{3}$ & 8.51 & 8.49 & 8.49 & 8.24 & 8.10 & 7.76 & 7.50 & 7.66 & 6.57 & 6.57 \\
\hline $\mathrm{MgO}$ & 2.50 & 2.44 & 2.18 & 2.03 & 2.01 & 1.94 & 1.86 & 1.67 & 1.59 & 1.18 \\
\hline $\mathrm{FeO}$ & 5.14 & 5.26 & 5.32 & 5.04 & 5.09 & 5.21 & 5.74 & 5.74 & 5.82 & 5.77 \\
\hline $\mathrm{CaO}$ & 2.04 & 1.83 & 1.76 & 1.64 & 1.61 & 1.63 & 1.58 & 1.43 & 1.34 & 1.20 \\
\hline $\mathrm{Na}_{2} \mathrm{O}$ & 2.19 & 2.18 & 2.18 & 2.16 & 2.07 & 2.02 & 1.95 & 1.95 & 1.70 & 1.72 \\
\hline $\mathrm{K}_{2} \mathrm{O}$ & 1.15 & 1.19 & 1.22 & 1.18 & 1.30 & 1.31 & 1.36 & 1.38 & 1.37 & 1.38 \\
\hline$N$ & 284 & 263 & 247 & 279 & 286 & 276 & 252 & 213 & 186 & 164 \\
\hline \multicolumn{11}{|c|}{$V_{p}=6.5 \mathrm{~km} / \mathrm{s}$} \\
\hline Mean & & & & & & & & & & \\
\hline $\mathrm{SiO}_{2}$ & 61.51 & 62.43 & 63.29 & 64.52 & 65.45 & 66.30 & 67.31 & 68.15 & 69.86 & 71.32 \\
\hline $\mathrm{Al}_{2} \mathrm{O}_{3}$ & 16.31 & 15.87 & 15.16 & 14.44 & 13.76 & 13.56 & 13.12 & 12.61 & 11.45 & 10.43 \\
\hline $\mathrm{MgO}$ & 4.42 & 4.12 & 4.19 & 4.22 & 4.22 & 3.70 & 3.56 & 3.39 & 3.25 & 3.10 \\
\hline $\mathrm{FeO}$ & 7.71 & 7.72 & 7.81 & 7.59 & 7.37 & 7.24 & 6.92 & 6.98 & 7.05 & 6.79 \\
\hline $\mathrm{CaO}$ & 3.90 & 3.72 & 3.51 & 3.16 & 3.08 & 3.14 & 2.97 & 2.87 & 2.56 & 2.55 \\
\hline $\mathrm{Na}_{2} \mathrm{O}$ & 3.95 & 3.89 & 3.76 & 3.67 & 3.58 & 3.51 & 3.45 & 3.34 & 3.12 & 2.94 \\
\hline $\mathrm{K}_{2} \mathrm{O}$ & 2.22 & 2.27 & 2.30 & 2.41 & 2.55 & 2.57 & 2.67 & 2.68 & 2.73 & 2.88 \\
\hline \multicolumn{11}{|l|}{ Std. Dev. } \\
\hline $\mathrm{SiO}_{2}$ & 9.55 & 10.19 & 10.09 & 11.00 & 11.24 & 10.72 & 10.17 & 10.14 & 10.21 & 10.17 \\
\hline $\mathrm{Al}_{2} \mathrm{O}_{3}$ & 8.44 & 8.55 & 8.43 & 9.22 & 9.22 & 8.78 & 8.66 & 8.54 & 8.54 & 8.49 \\
\hline $\mathrm{MgO}$ & 2.79 & 2.76 & 2.76 & 2.73 & 2.71 & 2.44 & 2.41 & 2.24 & 2.02 & 1.98 \\
\hline $\mathrm{FeO}$ & 5.63 & 5.60 & 5.71 & 5.66 & 5.70 & 5.60 & 5.29 & 5.31 & 5.15 & 5.08 \\
\hline $\mathrm{CaO}$ & 2.64 & 2.48 & 2.42 & 2.02 & 2.00 & 2.00 & 1.81 & 1.78 & 1.61 & 1.59 \\
\hline $\mathrm{Na}_{2} \mathrm{O}$ & 2.20 & 2.24 & 2.20 & 2.35 & 2.32 & 2.24 & 2.21 & 2.19 & 2.24 & 2.18 \\
\hline $\mathrm{K}_{2} \mathrm{O}$ & 1.07 & 1.09 & 1.11 & 1.09 & 1.17 & 1.20 & 1.19 & 1.20 & 1.21 & 1.28 \\
\hline$N$ & 371 & 354 & 354 & 337 & 343 & 301 & 272 & 271 & 284 & 295 \\
\hline
\end{tabular}


Table A.2: continued

\begin{tabular}{|c|c|c|c|c|c|c|c|c|c|c|}
\hline Depth $(\mathrm{km})$ & 5 & 10 & 15 & 20 & 25 & 30 & 35 & 40 & 45 & 50 \\
\hline $\mathrm{T}(\mathrm{C})$ & 61.99 & 101.36 & 136.10 & 168.04 & 198.27 & 227.47 & 256.05 & 284.25 & 312.21 & 340.04 \\
\hline $\mathrm{P}$ (kbar) & 1.62 & 3.23 & 4.85 & 6.47 & 8.09 & 9.70 & 11.32 & 12.94 & 14.55 & 16.17 \\
\hline \multicolumn{11}{|c|}{$V_{p}=6.6 \mathrm{~km} / \mathrm{s}$} \\
\hline Mean & & & & & & & & & & \\
\hline $\mathrm{SiO}_{2}$ & 59.03 & 59.36 & 59.54 & 59.42 & 59.38 & 60.26 & 60.79 & 62.73 & 63.94 & 64.87 \\
\hline $\mathrm{Al}_{2} \mathrm{O}_{3}$ & 17.06 & 16.87 & 16.94 & 17.30 & 17.48 & 16.92 & 16.84 & 15.57 & 14.81 & 14.20 \\
\hline $\mathrm{MgO}$ & 5.45 & 5.26 & 5.17 & 5.00 & 4.72 & 4.62 & 4.34 & 4.23 & 4.21 & 4.17 \\
\hline $\mathrm{FeO}$ & 8.08 & 8.18 & 8.03 & 7.97 & 8.09 & 8.06 & 8.06 & 7.85 & 7.78 & 7.51 \\
\hline $\mathrm{CaO}$ & 4.58 & 4.51 & 4.37 & 4.23 & 4.09 & 3.89 & 3.66 & 3.47 & 3.07 & 3.02 \\
\hline $\mathrm{Na}_{2} \mathrm{O}$ & 3.92 & 3.90 & 3.96 & 4.08 & 4.17 & 4.10 & 4.13 & 3.87 & 3.79 & 3.70 \\
\hline $\mathrm{K}_{2} \mathrm{O}$ & 1.90 & 1.93 & 1.99 & 2.03 & 2.08 & 2.15 & 2.19 & 2.29 & 2.41 & 2.54 \\
\hline \multicolumn{11}{|l|}{ Std. Dev. } \\
\hline $\mathrm{SiO}_{2}$ & 9.91 & 10.24 & 10.27 & 10.18 & 10.36 & 10.28 & 10.27 & 10.71 & 11.57 & 11.70 \\
\hline $\mathrm{Al}_{2} \mathrm{O}_{3}$ & 8.42 & 8.60 & 8.55 & 8.59 & 8.64 & 8.68 & 8.67 & 8.76 & 9.55 & 9.55 \\
\hline $\mathrm{MgO}$ & 3.40 & 3.28 & 3.24 & 3.28 & 3.10 & 3.01 & 2.81 & 2.78 & 2.69 & 2.71 \\
\hline $\mathrm{FeO}$ & 5.63 & 5.63 & 5.77 & 5.79 & 5.73 & 5.75 & 5.68 & 5.65 & 5.65 & 5.67 \\
\hline $\mathrm{CaO}$ & 2.93 & 2.91 & 2.92 & 2.76 & 2.69 & 2.58 & 2.43 & 2.42 & 2.00 & 1.96 \\
\hline $\mathrm{Na}_{2} \mathrm{O}$ & 2.20 & 2.27 & 2.25 & 2.22 & 2.22 & 2.24 & 2.23 & 2.28 & 2.41 & 2.41 \\
\hline $\mathrm{K}_{2} \mathrm{O}$ & 1.08 & 1.06 & 1.10 & 1.10 & 1.09 & 1.10 & 1.09 & 1.09 & 1.13 & 1.17 \\
\hline$N$ & 489 & 476 & 468 & 438 & 426 & 395 & 387 & 377 & 358 & 353 \\
\hline \multicolumn{11}{|c|}{$V_{p}=6.7 \mathrm{~km} / \mathrm{s}$} \\
\hline Mean & & & & & & & & & & \\
\hline $\mathrm{SiO}_{2}$ & 56.54 & 56.89 & 56.92 & 56.79 & 57.29 & 58.11 & 58.39 & 58.37 & 58.22 & 58.14 \\
\hline $\mathrm{Al}_{2} \mathrm{O}_{3}$ & 17.89 & 17.76 & 17.92 & 18.32 & 18.20 & 17.61 & 17.48 & 17.66 & 17.93 & 18.08 \\
\hline $\mathrm{MgO}$ & 6.53 & 6.28 & 6.00 & 5.69 & 5.53 & 5.54 & 5.43 & 5.25 & 5.06 & 4.89 \\
\hline $\mathrm{FeO}$ & 8.38 & 8.39 & 8.35 & 8.42 & 8.18 & 8.23 & 8.24 & 8.25 & 8.44 & 8.52 \\
\hline $\mathrm{CaO}$ & 5.19 & 5.13 & 5.17 & 4.96 & 4.92 & 4.60 & 4.43 & 4.31 & 4.06 & 3.95 \\
\hline $\mathrm{Na}_{2} \mathrm{O}$ & 3.90 & 3.92 & 3.96 & 4.11 & 4.10 & 4.05 & 4.09 & 4.17 & 4.29 & 4.38 \\
\hline $\mathrm{K}_{2} \mathrm{O}$ & 1.58 & 1.65 & 1.68 & 1.72 & 1.79 & 1.88 & 1.95 & 2.00 & 2.02 & 2.06 \\
\hline \multicolumn{11}{|l|}{ Std. Dev. } \\
\hline $\mathrm{SiO}_{2}$ & 10.09 & 10.17 & 10.21 & 10.11 & 10.18 & 10.62 & 10.93 & 11.13 & 11.14 & 11.34 \\
\hline $\mathrm{Al}_{2} \mathrm{O}_{3}$ & 8.41 & 8.44 & 8.62 & 8.28 & 8.37 & 8.77 & 8.86 & 8.92 & 8.87 & 8.90 \\
\hline $\mathrm{MgO}$ & 3.61 & 3.46 & 3.39 & 3.42 & 3.38 & 3.36 & 3.35 & 3.19 & 3.25 & 3.09 \\
\hline $\mathrm{FeO}$ & 5.43 & 5.53 & 5.41 & 5.56 & 5.44 & 5.62 & 5.69 & 5.84 & 5.93 & 5.95 \\
\hline $\mathrm{CaO}$ & 3.34 & 3.20 & 3.18 & 3.07 & 3.02 & 2.92 & 2.86 & 2.88 & 2.72 & 2.61 \\
\hline $\mathrm{Na}_{2} \mathrm{O}$ & 2.31 & 2.30 & 2.35 & 2.24 & 2.25 & 2.30 & 2.36 & 2.36 & 2.33 & 2.33 \\
\hline $\mathrm{K}_{2} \mathrm{O}$ & 0.99 & 1.01 & 1.02 & 1.05 & 1.04 & 1.06 & 1.06 & 1.07 & 1.09 & 1.10 \\
\hline$N$ & 583 & 578 & 568 & 558 & 529 & 543 & 521 & 489 & 469 & 454 \\
\hline
\end{tabular}


Table A.2: continued

\begin{tabular}{|c|c|c|c|c|c|c|c|c|c|c|}
\hline Depth $(\mathrm{km})$ & 5 & 10 & 15 & 20 & 25 & 30 & 35 & 40 & 45 & 50 \\
\hline $\mathrm{T}(\mathrm{C})$ & 61.99 & 101.36 & 136.10 & 168.04 & 198.27 & 227.47 & 256.05 & 284.25 & 312.21 & 340.04 \\
\hline $\mathrm{P}$ (kbar) & 1.62 & 3.23 & 4.85 & 6.47 & 8.09 & 9.70 & 11.32 & 12.94 & 14.55 & 16.17 \\
\hline \multicolumn{11}{|c|}{$V_{p}=6.8 \mathrm{~km} / \mathrm{s}$} \\
\hline Mean & & & & & & & & & & \\
\hline $\mathrm{SiO}_{2}$ & 55.02 & 55.13 & 55.32 & 55.60 & 55.96 & 55.94 & 56.21 & 56.16 & 56.24 & 56.16 \\
\hline $\mathrm{Al}_{2} \mathrm{O}_{3}$ & 18.31 & 18.29 & 18.27 & 18.13 & 17.96 & 18.22 & 18.06 & 18.29 & 18.45 & 18.77 \\
\hline $\mathrm{MgO}$ & 7.64 & 7.57 & 7.43 & 7.26 & 7.16 & 6.76 & 6.62 & 6.20 & 5.83 & 5.69 \\
\hline $\mathrm{FeO}$ & 7.98 & 8.00 & 7.98 & 8.04 & 8.15 & 8.21 & 8.42 & 8.60 & 8.65 & 8.62 \\
\hline $\mathrm{CaO}$ & 5.78 & 5.67 & 5.62 & 5.59 & 5.34 & 5.35 & 5.06 & 5.05 & 5.01 & 4.76 \\
\hline $\mathrm{Na}_{2} \mathrm{O}$ & 3.83 & 3.87 & 3.88 & 3.86 & 3.88 & 3.95 & 4.00 & 4.07 & 4.14 & 4.28 \\
\hline $\mathrm{K}_{2} \mathrm{O}$ & 1.45 & 1.49 & 1.50 & 1.53 & 1.56 & 1.58 & 1.64 & 1.63 & 1.69 & 1.74 \\
\hline \multicolumn{11}{|l|}{ Std. Dev. } \\
\hline $\mathrm{SiO}_{2}$ & 9.98 & 9.93 & 10.07 & 10.37 & 10.33 & 10.36 & 10.68 & 10.65 & 10.61 & 10.77 \\
\hline $\mathrm{Al}_{2} \mathrm{O}_{3}$ & 8.42 & 8.37 & 8.47 & 8.56 & 8.44 & 8.51 & 8.62 & 8.65 & 8.39 & 8.42 \\
\hline $\mathrm{MgO}$ & 3.91 & 3.88 & 3.81 & 3.65 & 3.63 & 3.57 & 3.61 & 3.41 & 3.39 & 3.35 \\
\hline $\mathrm{FeO}$ & 5.32 & 5.34 & 5.34 & 5.31 & 5.37 & 5.40 & 5.49 & 5.58 & 5.71 & 5.73 \\
\hline $\mathrm{CaO}$ & 3.75 & 3.63 & 3.57 & 3.53 & 3.45 & 3.44 & 3.20 & 3.15 & 3.16 & 3.02 \\
\hline $\mathrm{Na}_{2} \mathrm{O}$ & 2.37 & 2.33 & 2.35 & 2.39 & 2.34 & 2.33 & 2.34 & 2.36 & 2.30 & 2.27 \\
\hline $\mathrm{K}_{2} \mathrm{O}$ & 0.90 & 0.92 & 0.93 & 0.94 & 0.95 & 0.96 & 0.99 & 1.01 & 1.05 & 1.04 \\
\hline$N$ & 666 & 674 & 671 & 663 & 638 & 645 & 600 & 602 & 593 & 576 \\
\hline \multicolumn{11}{|c|}{$V_{p}=6.9 \mathrm{~km} / \mathrm{s}$} \\
\hline Mean & & & & & & & & & & \\
\hline $\mathrm{SiO}_{2}$ & 54.59 & 54.81 & 54.88 & 55.41 & 55.30 & 55.22 & 55.34 & 55.68 & 55.97 & 55.94 \\
\hline $\mathrm{Al}_{2} \mathrm{O}_{3}$ & 17.62 & 17.69 & 17.79 & 17.41 & 17.62 & 17.82 & 18.08 & 17.91 & 17.94 & 17.98 \\
\hline $\mathrm{MgO}$ & 9.20 & 8.99 & 8.89 & 8.70 & 8.50 & 8.32 & 7.73 & 7.58 & 7.40 & 7.23 \\
\hline $\mathrm{FeO}$ & 7.09 & 6.95 & 6.97 & 7.05 & 7.29 & 7.52 & 7.76 & 7.92 & 7.67 & 7.93 \\
\hline $\mathrm{CaO}$ & 6.53 & 6.58 & 6.36 & 6.41 & 6.20 & 5.97 & 5.91 & 5.71 & 5.75 & 5.55 \\
\hline $\mathrm{Na}_{2} \mathrm{O}$ & 3.53 & 3.53 & 3.61 & 3.52 & 3.60 & 3.68 & 3.74 & 3.75 & 3.76 & 3.83 \\
\hline $\mathrm{K}_{2} \mathrm{O}$ & 1.45 & 1.46 & 1.51 & 1.51 & 1.50 & 1.48 & 1.45 & 1.47 & 1.53 & 1.55 \\
\hline \multicolumn{11}{|l|}{ Std. Dev. } \\
\hline $\mathrm{SiO}_{2}$ & 9.22 & 9.12 & 9.20 & 9.44 & 9.56 & 9.47 & 9.55 & 9.75 & 9.96 & 10.03 \\
\hline $\mathrm{Al}_{2} \mathrm{O}_{3}$ & 8.13 & 8.09 & 8.07 & 8.28 & 8.30 & 8.15 & 8.16 & 8.30 & 8.35 & 8.35 \\
\hline $\mathrm{MgO}$ & 4.83 & 4.73 & 4.65 & 4.56 & 4.46 & 4.26 & 3.89 & 3.88 & 3.77 & 3.72 \\
\hline $\mathrm{FeO}$ & 4.83 & 4.79 & 4.85 & 4.72 & 4.98 & 5.02 & 5.19 & 5.13 & 5.06 & 5.10 \\
\hline $\mathrm{CaO}$ & 4.15 & 4.12 & 4.03 & 4.01 & 3.92 & 3.78 & 3.70 & 3.59 & 3.52 & 3.47 \\
\hline $\mathrm{Na}_{2} \mathrm{O}$ & 2.19 & 2.17 & 2.18 & 2.22 & 2.23 & 2.21 & 2.22 & 2.25 & 2.26 & 2.23 \\
\hline $\mathrm{K}_{2} \mathrm{O}$ & 0.90 & 0.90 & 0.90 & 0.90 & 0.91 & 0.90 & 0.90 & 0.92 & 0.93 & 0.94 \\
\hline$N$ & 676 & 699 & 671 & 690 & 697 & 686 & 677 & 676 & 679 & 649 \\
\hline
\end{tabular}


Table A.2: continued

\begin{tabular}{|c|c|c|c|c|c|c|c|c|c|c|}
\hline Depth $(\mathrm{km})$ & 5 & 10 & 15 & 20 & 25 & 30 & 35 & 40 & 45 & 50 \\
\hline $\mathrm{T}(\mathrm{C})$ & 61.99 & 101.36 & 136.10 & 168.04 & 198.27 & 227.47 & 256.05 & 284.25 & 312.21 & 340.04 \\
\hline $\mathrm{P}$ (kbar) & 1.62 & 3.23 & 4.85 & 6.47 & 8.09 & 9.70 & 11.32 & 12.94 & 14.55 & 16.17 \\
\hline \multicolumn{11}{|c|}{$V_{p}=7.0 \mathrm{~km} / \mathrm{s}$} \\
\hline Mean & & & & & & & & & & \\
\hline $\mathrm{SiO}_{2}$ & 53.54 & 53.59 & 53.84 & 54.13 & 54.17 & 54.30 & 54.65 & 54.70 & 55.02 & 55.30 \\
\hline $\mathrm{Al}_{2} \mathrm{O}_{3}$ & 17.10 & 17.30 & 17.26 & 17.22 & 17.39 & 17.44 & 17.49 & 17.57 & 17.51 & 17.38 \\
\hline $\mathrm{MgO}$ & 10.75 & 10.43 & 10.28 & 10.03 & 9.84 & 9.77 & 9.41 & 9.40 & 9.07 & 8.86 \\
\hline $\mathrm{FeO}$ & 6.93 & 6.84 & 6.81 & 6.86 & 6.97 & 7.04 & 6.88 & 6.94 & 6.98 & 7.08 \\
\hline $\mathrm{CaO}$ & 7.17 & 7.23 & 7.18 & 7.09 & 6.88 & 6.61 & 6.70 & 6.37 & 6.39 & 6.39 \\
\hline $\mathrm{Na}_{2} \mathrm{O}$ & 3.24 & 3.29 & 3.30 & 3.32 & 3.39 & 3.45 & 3.45 & 3.54 & 3.54 & 3.50 \\
\hline $\mathrm{K}_{2} \mathrm{O}$ & 1.29 & 1.32 & 1.35 & 1.37 & 1.38 & 1.40 & 1.43 & 1.48 & 1.50 & 1.49 \\
\hline \multicolumn{11}{|l|}{ Std. Dev. } \\
\hline $\mathrm{SiO}_{2}$ & 8.71 & 8.79 & 8.71 & 8.80 & 9.04 & 9.18 & 9.15 & 9.27 & 9.41 & 9.47 \\
\hline $\mathrm{Al}_{2} \mathrm{O}_{3}$ & 7.81 & 7.81 & 7.78 & 7.81 & 7.98 & 7.99 & 7.96 & 8.02 & 8.16 & 8.14 \\
\hline $\mathrm{MgO}$ & 5.43 & 5.42 & 5.42 & 5.40 & 5.26 & 5.11 & 5.01 & 4.95 & 4.74 & 4.56 \\
\hline $\mathrm{FeO}$ & 4.72 & 4.63 & 4.54 & 4.63 & 4.62 & 4.67 & 4.63 & 4.64 & 4.55 & 4.59 \\
\hline $\mathrm{CaO}$ & 4.64 & 4.60 & 4.57 & 4.58 & 4.30 & 4.18 & 4.21 & 4.06 & 3.95 & 3.92 \\
\hline $\mathrm{Na}_{2} \mathrm{O}$ & 2.03 & 2.04 & 2.03 & 2.04 & 2.07 & 2.09 & 2.09 & 2.11 & 2.15 & 2.14 \\
\hline $\mathrm{K}_{2} \mathrm{O}$ & 0.87 & 0.86 & 0.87 & 0.88 & 0.89 & 0.89 & 0.89 & 0.90 & 0.90 & 0.89 \\
\hline$N$ & 627 & 626 & 635 & 642 & 664 & 669 & 719 & 709 & 715 & 724 \\
\hline \multicolumn{11}{|c|}{$V_{p}=7.1 \mathrm{~km} / \mathrm{s}$} \\
\hline Mean & & & & & & & & & & \\
\hline $\mathrm{SiO}_{2}$ & 51.39 & 51.52 & 51.82 & 52.03 & 52.22 & 52.43 & 52.84 & 53.12 & 53.36 & 54.01 \\
\hline $\mathrm{Al}_{2} \mathrm{O}_{3}$ & 17.68 & 17.69 & 17.70 & 17.76 & 17.72 & 17.77 & 17.55 & 17.58 & 17.40 & 17.13 \\
\hline $\mathrm{MgO}$ & 12.04 & 12.06 & 11.57 & 11.32 & 11.21 & 10.99 & 10.95 & 10.67 & 10.39 & 10.25 \\
\hline $\mathrm{FeO}$ & 7.10 & 7.14 & 7.27 & 7.22 & 7.15 & 7.03 & 6.98 & 6.84 & 7.12 & 7.08 \\
\hline $\mathrm{CaO}$ & 7.38 & 7.16 & 7.19 & 7.20 & 7.20 & 7.22 & 7.09 & 7.13 & 7.09 & 6.89 \\
\hline $\mathrm{Na}_{2} \mathrm{O}$ & 3.30 & 3.33 & 3.33 & 3.35 & 3.34 & 3.37 & 3.35 & 3.37 & 3.35 & 3.32 \\
\hline $\mathrm{K}_{2} \mathrm{O}$ & 1.12 & 1.13 & 1.13 & 1.14 & 1.16 & 1.21 & 1.26 & 1.31 & 1.31 & 1.34 \\
\hline \multicolumn{11}{|l|}{ Std. Dev. } \\
\hline $\mathrm{SiO}_{2}$ & 8.68 & 8.92 & 9.06 & 9.05 & 9.06 & 9.21 & 9.10 & 9.17 & 9.15 & 9.38 \\
\hline $\mathrm{Al}_{2} \mathrm{O}_{3}$ & 7.71 & 7.80 & 7.88 & 7.82 & 7.76 & 7.92 & 7.85 & 7.82 & 7.84 & 7.91 \\
\hline $\mathrm{MgO}$ & 5.85 & 5.77 & 5.54 & 5.37 & 5.36 & 5.46 & 5.50 & 5.48 & 5.42 & 5.29 \\
\hline $\mathrm{FeO}$ & 4.99 & 5.02 & 5.00 & 4.96 & 4.85 & 4.81 & 4.74 & 4.73 & 4.80 & 4.79 \\
\hline $\mathrm{CaO}$ & 5.07 & 5.04 & 4.86 & 4.75 & 4.70 & 4.67 & 4.67 & 4.63 & 4.57 & 4.35 \\
\hline $\mathrm{Na}_{2} \mathrm{O}$ & 2.05 & 2.09 & 2.08 & 2.07 & 2.06 & 2.07 & 2.04 & 2.03 & 2.03 & 2.04 \\
\hline $\mathrm{K}_{2} \mathrm{O}$ & 0.80 & 0.79 & 0.79 & 0.78 & 0.78 & 0.82 & 0.85 & 0.84 & 0.86 & 0.88 \\
\hline$N$ & 567 & 569 & 582 & 582 & 606 & 628 & 674 & 676 & 680 & 690 \\
\hline
\end{tabular}


Table A.2: continued

\begin{tabular}{|c|c|c|c|c|c|c|c|c|c|c|}
\hline Depth $(\mathrm{km})$ & 5 & 10 & 15 & 20 & 25 & 30 & 35 & 40 & 45 & 50 \\
\hline $\mathrm{T}(\mathrm{C})$ & 61.99 & 101.36 & 136.10 & 168.04 & 198.27 & 227.47 & 256.05 & 284.25 & 312.21 & 340.04 \\
\hline $\mathrm{P}$ (kbar) & 1.62 & 3.23 & 4.85 & 6.47 & 8.09 & 9.70 & 11.32 & 12.94 & 14.55 & 16.17 \\
\hline \multicolumn{11}{|c|}{$V_{p}=7.2 \mathrm{~km} / \mathrm{s}$} \\
\hline Mean & & & & & & & & & & \\
\hline $\mathrm{SiO}_{2}$ & 50.18 & 50.52 & 50.56 & 50.77 & 51.11 & 51.32 & 51.32 & 51.63 & 51.63 & 51.95 \\
\hline $\mathrm{Al}_{2} \mathrm{O}_{3}$ & 17.91 & 17.67 & 17.61 & 17.54 & 17.49 & 17.53 & 17.56 & 17.64 & 17.79 & 17.76 \\
\hline $\mathrm{MgO}$ & 12.75 & 12.80 & 12.82 & 12.82 & 12.60 & 12.30 & 12.13 & 11.82 & 11.69 & 11.43 \\
\hline $\mathrm{FeO}$ & 7.25 & 7.27 & 7.35 & 7.25 & 7.01 & 7.02 & 7.22 & 7.07 & 7.19 & 7.09 \\
\hline $\mathrm{CaO}$ & 7.54 & 7.45 & 7.39 & 7.36 & 7.55 & 7.56 & 7.46 & 7.52 & 7.32 & 7.36 \\
\hline $\mathrm{Na}_{2} \mathrm{O}$ & 3.33 & 3.26 & 3.25 & 3.24 & 3.19 & 3.21 & 3.25 & 3.24 & 3.31 & 3.30 \\
\hline $\mathrm{K}_{2} \mathrm{O}$ & 1.05 & 1.03 & 1.02 & 1.04 & 1.06 & 1.08 & 1.08 & 1.08 & 1.08 & 1.12 \\
\hline \multicolumn{11}{|l|}{ Std. Dev. } \\
\hline $\mathrm{SiO}_{2}$ & 8.63 & 8.62 & 8.63 & 8.49 & 8.60 & 8.82 & 8.93 & 9.02 & 9.21 & 9.38 \\
\hline $\mathrm{Al}_{2} \mathrm{O}_{3}$ & 7.81 & 7.75 & 7.72 & 7.59 & 7.49 & 7.66 & 7.81 & 7.81 & 7.89 & 7.96 \\
\hline $\mathrm{MgO}$ & 6.22 & 6.16 & 6.04 & 5.96 & 5.97 & 5.98 & 5.87 & 5.78 & 5.63 & 5.57 \\
\hline $\mathrm{FeO}$ & 4.87 & 4.94 & 4.94 & 5.00 & 4.99 & 4.99 & 5.07 & 5.04 & 4.94 & 4.93 \\
\hline $\mathrm{CaO}$ & 5.20 & 5.20 & 5.11 & 5.03 & 5.10 & 5.06 & 5.01 & 4.97 & 4.90 & 4.78 \\
\hline $\mathrm{Na}_{2} \mathrm{O}$ & 2.04 & 2.02 & 2.02 & 1.97 & 1.99 & 2.01 & 2.03 & 2.01 & 2.01 & 2.06 \\
\hline $\mathrm{K}_{2} \mathrm{O}$ & 0.76 & 0.75 & 0.74 & 0.74 & 0.75 & 0.77 & 0.74 & 0.76 & 0.75 & 0.78 \\
\hline$N$ & 483 & 508 & 521 & 550 & 570 & 575 & 573 & 591 & 592 & 597 \\
\hline \multicolumn{11}{|c|}{$V_{p}=7.3 \mathrm{~km} / \mathrm{s}$} \\
\hline Mean & & & & & & & & & & \\
\hline $\mathrm{SiO}_{2}$ & 48.73 & 48.98 & 49.31 & 49.49 & 49.66 & 50.09 & 50.17 & 50.53 & 50.82 & 50.98 \\
\hline $\mathrm{Al}_{2} \mathrm{O}_{3}$ & 18.69 & 18.45 & 18.25 & 18.08 & 18.13 & 17.80 & 17.89 & 17.74 & 17.64 & 17.70 \\
\hline $\mathrm{MgO}$ & 12.76 & 12.88 & 13.11 & 13.10 & 12.81 & 12.80 & 12.48 & 12.51 & 12.68 & 12.53 \\
\hline $\mathrm{FeO}$ & 6.83 & 6.87 & 6.72 & 6.80 & 6.92 & 7.01 & 6.99 & 7.02 & 6.83 & 6.66 \\
\hline $\mathrm{CaO}$ & 8.84 & 8.69 & 8.50 & 8.47 & 8.34 & 8.23 & 8.32 & 8.10 & 7.90 & 7.98 \\
\hline $\mathrm{Na}_{2} \mathrm{O}$ & 3.25 & 3.22 & 3.19 & 3.15 & 3.20 & 3.14 & 3.17 & 3.14 & 3.14 & 3.14 \\
\hline $\mathrm{K}_{2} \mathrm{O}$ & 0.91 & 0.92 & 0.94 & 0.92 & 0.94 & 0.95 & 0.99 & 0.97 & 1.01 & 1.02 \\
\hline \multicolumn{11}{|l|}{ Std. Dev. } \\
\hline $\mathrm{SiO}_{2}$ & 8.06 & 8.11 & 8.18 & 8.22 & 8.45 & 8.41 & 8.69 & 8.55 & 8.58 & 8.49 \\
\hline $\mathrm{Al}_{2} \mathrm{O}_{3}$ & 7.82 & 7.85 & 7.71 & 7.68 & 7.78 & 7.72 & 7.93 & 7.80 & 7.74 & 7.69 \\
\hline $\mathrm{MgO}$ & 6.72 & 6.67 & 6.78 & 6.79 & 6.72 & 6.64 & 6.33 & 6.16 & 6.02 & 6.07 \\
\hline $\mathrm{FeO}$ & 4.45 & 4.36 & 4.52 & 4.67 & 4.76 & 4.79 & 4.84 & 4.77 & 4.78 & 4.76 \\
\hline $\mathrm{CaO}$ & 5.55 & 5.46 & 5.64 & 5.65 & 5.66 & 5.53 & 5.40 & 5.20 & 5.14 & 5.21 \\
\hline $\mathrm{Na}_{2} \mathrm{O}$ & 1.91 & 1.91 & 1.90 & 1.88 & 1.88 & 1.90 & 1.93 & 1.93 & 1.92 & 1.92 \\
\hline $\mathrm{K}_{2} \mathrm{O}$ & 0.71 & 0.72 & 0.72 & 0.70 & 0.72 & 0.71 & 0.73 & 0.73 & 0.73 & 0.72 \\
\hline$N$ & 420 & 435 & 461 & 480 & 469 & 490 & 477 & 510 & 533 & 573 \\
\hline
\end{tabular}


Table A.2: continued

\begin{tabular}{|c|c|c|c|c|c|c|c|c|c|c|}
\hline Depth $(\mathrm{km})$ & 5 & 10 & 15 & 20 & 25 & 30 & 35 & 40 & 45 & 50 \\
\hline $\mathrm{T}(\mathrm{C})$ & 61.99 & 101.36 & 136.10 & 168.04 & 198.27 & 227.47 & 256.05 & 284.25 & 312.21 & 340.04 \\
\hline$P$ (kbar) & 1.62 & 3.23 & 4.85 & 6.47 & 8.09 & 9.70 & 11.32 & 12.94 & 14.55 & 16.17 \\
\hline \multicolumn{11}{|c|}{$V_{p}=7.4 \mathrm{~km} / \mathrm{s}$} \\
\hline Mean & & & & & & & & & & \\
\hline $\mathrm{SiO}_{2}$ & 47.33 & 47.34 & 47.49 & 47.66 & 47.83 & 48.27 & 48.86 & 49.30 & 49.95 & 50.07 \\
\hline $\mathrm{Al}_{2} \mathrm{O}_{3}$ & 19.27 & 19.46 & 19.39 & 19.47 & 19.34 & 18.87 & 18.61 & 18.19 & 17.94 & 17.78 \\
\hline $\mathrm{MgO}$ & 13.71 & 13.39 & 13.26 & 13.00 & 12.95 & 13.25 & 12.99 & 12.98 & 13.07 & 13.07 \\
\hline $\mathrm{FeO}$ & 5.96 & 6.01 & 6.06 & 6.04 & 6.24 & 6.27 & 6.32 & 6.41 & 6.17 & 6.40 \\
\hline $\mathrm{CaO}$ & 9.72 & 9.71 & 9.69 & 9.73 & 9.53 & 9.30 & 9.25 & 9.18 & 8.90 & 8.72 \\
\hline $\mathrm{Na}_{2} \mathrm{O}$ & 3.19 & 3.25 & 3.24 & 3.24 & 3.26 & 3.18 & 3.12 & 3.05 & 3.03 & 3.02 \\
\hline $\mathrm{K}_{2} \mathrm{O}$ & 0.84 & 0.85 & 0.86 & 0.86 & 0.87 & 0.88 & 0.87 & 0.91 & 0.96 & 0.95 \\
\hline \multicolumn{11}{|l|}{ Std. Dev. } \\
\hline $\mathrm{SiO}_{2}$ & 7.32 & 7.42 & 7.40 & 7.47 & 7.59 & 7.51 & 7.67 & 7.63 & 7.78 & 7.77 \\
\hline $\mathrm{Al}_{2} \mathrm{O}_{3}$ & 7.35 & 7.31 & 7.23 & 7.23 & 7.42 & 7.32 & 7.35 & 7.37 & 7.27 & 7.28 \\
\hline $\mathrm{MgO}$ & 7.47 & 7.41 & 7.45 & 7.35 & 7.28 & 7.12 & 6.81 & 6.79 & 6.76 & 6.75 \\
\hline $\mathrm{FeO}$ & 4.09 & 4.06 & 4.12 & 4.07 & 4.12 & 4.14 & 4.09 & 4.16 & 4.33 & 4.45 \\
\hline $\mathrm{CaO}$ & 5.81 & 5.82 & 5.87 & 5.84 & 5.79 & 5.73 & 5.54 & 5.54 & 5.47 & 5.58 \\
\hline $\mathrm{Na}_{2} \mathrm{O}$ & 1.82 & 1.81 & 1.79 & 1.81 & 1.78 & 1.74 & 1.78 & 1.76 & 1.76 & 1.75 \\
\hline $\mathrm{K}_{2} \mathrm{O}$ & 0.69 & 0.70 & 0.72 & 0.72 & 0.70 & 0.68 & 0.70 & 0.71 & 0.73 & 0.73 \\
\hline$N$ & 349 & 347 & 352 & 352 & 354 & 383 & 400 & 438 & 463 & 477 \\
\hline \multicolumn{11}{|c|}{$V_{p}=7.5 \mathrm{~km} / \mathrm{s}$} \\
\hline Mean & & & & & & & & & & \\
\hline $\mathrm{SiO}_{2}$ & 47.40 & 47.46 & 47.90 & 48.22 & 48.47 & 48.35 & 48.37 & 48.14 & 48.07 & 48.17 \\
\hline $\mathrm{Al}_{2} \mathrm{O}_{3}$ & 18.37 & 18.49 & 18.26 & 18.08 & 17.77 & 17.95 & 17.98 & 18.35 & 18.66 & 18.63 \\
\hline $\mathrm{MgO}$ & 15.02 & 14.62 & 14.15 & 14.28 & 14.36 & 14.23 & 14.54 & 14.26 & 13.79 & 13.59 \\
\hline $\mathrm{FeO}$ & 5.82 & 5.84 & 6.00 & 6.02 & 6.00 & 5.98 & 5.97 & 5.97 & 5.99 & 6.08 \\
\hline $\mathrm{CaO}$ & 9.61 & 9.79 & 9.97 & 9.68 & 9.74 & 9.82 & 9.43 & 9.43 & 9.57 & 9.60 \\
\hline $\mathrm{Na}_{2} \mathrm{O}$ & 2.97 & 2.99 & 2.91 & 2.91 & 2.83 & 2.85 & 2.90 & 3.01 & 3.07 & 3.07 \\
\hline $\mathrm{K}_{2} \mathrm{O}$ & 0.82 & 0.83 & 0.82 & 0.83 & 0.83 & 0.82 & 0.82 & 0.86 & 0.86 & 0.87 \\
\hline \multicolumn{11}{|l|}{ Std. Dev. } \\
\hline $\mathrm{SiO}_{2}$ & 7.23 & 7.09 & 7.07 & 7.26 & 7.13 & 7.18 & 7.04 & 7.13 & 7.01 & 7.05 \\
\hline $\mathrm{Al}_{2} \mathrm{O}_{3}$ & 6.63 & 6.54 & 6.65 & 6.66 & 6.68 & 6.83 & 6.82 & 6.82 & 6.70 & 6.71 \\
\hline $\mathrm{MgO}$ & 8.03 & 8.03 & 7.70 & 7.52 & 7.38 & 7.33 & 7.45 & 7.47 & 7.64 & 7.62 \\
\hline $\mathrm{FeO}$ & 3.90 & 3.99 & 4.10 & 4.07 & 4.05 & 4.06 & 3.93 & 4.02 & 4.01 & 4.04 \\
\hline $\mathrm{CaO}$ & 6.25 & 6.31 & 6.15 & 6.01 & 5.94 & 5.90 & 5.88 & 5.99 & 6.02 & 5.97 \\
\hline $\mathrm{Na}_{2} \mathrm{O}$ & 1.82 & 1.80 & 1.80 & 1.82 & 1.81 & 1.80 & 1.76 & 1.75 & 1.73 & 1.74 \\
\hline $\mathrm{K}_{2} \mathrm{O}$ & 0.66 & 0.67 & 0.68 & 0.68 & 0.67 & 0.67 & 0.67 & 0.70 & 0.71 & 0.72 \\
\hline$N$ & 255 & 267 & 278 & 288 & 311 & 316 & 334 & 336 & 338 & 343 \\
\hline
\end{tabular}


Table A.2: continued

\begin{tabular}{|c|c|c|c|c|c|c|c|c|c|c|}
\hline Depth $(\mathrm{km})$ & 5 & 10 & 15 & 20 & 25 & 30 & 35 & 40 & 45 & 50 \\
\hline $\mathrm{T}(\mathrm{C})$ & 61.99 & 101.36 & 136.10 & 168.04 & 198.27 & 227.47 & 256.05 & 284.25 & 312.21 & 340.04 \\
\hline P (kbar) & 1.62 & 3.23 & 4.85 & 6.47 & 8.09 & 9.70 & 11.32 & 12.94 & 14.55 & 16.17 \\
\hline \multicolumn{11}{|c|}{$V_{p}=7.6 \mathrm{~km} / \mathrm{s}$} \\
\hline Mean & & & & & & & & & & \\
\hline $\mathrm{SiO}_{2}$ & 45.58 & 45.74 & 45.86 & 46.03 & 46.49 & 46.64 & 46.48 & 46.35 & 46.60 & 46.85 \\
\hline $\mathrm{Al}_{2} \mathrm{O}_{3}$ & 19.83 & 19.55 & 19.49 & 19.18 & 18.84 & 18.83 & 18.91 & 18.98 & 18.80 & 18.68 \\
\hline $\mathrm{MgO}$ & 14.39 & 14.67 & 14.70 & 14.95 & 14.97 & 14.71 & 14.97 & 14.96 & 14.77 & 14.77 \\
\hline $\mathrm{FeO}$ & 5.05 & 5.07 & 5.11 & 5.35 & 5.33 & 5.37 & 5.36 & 5.39 & 5.53 & 5.48 \\
\hline $\mathrm{CaO}$ & 11.10 & 10.99 & 10.86 & 10.55 & 10.54 & 10.68 & 10.44 & 10.46 & 10.49 & 10.42 \\
\hline $\mathrm{Na}_{2} \mathrm{O}$ & 3.16 & 3.10 & 3.11 & 3.08 & 2.98 & 2.95 & 3.00 & 3.02 & 2.98 & 2.96 \\
\hline $\mathrm{K}_{2} \mathrm{O}$ & 0.91 & 0.89 & 0.89 & 0.88 & 0.86 & 0.84 & 0.85 & 0.85 & 0.84 & 0.86 \\
\hline \multicolumn{11}{|l|}{ Std. Dev. } \\
\hline $\mathrm{SiO}_{2}$ & 7.32 & 7.40 & 7.45 & 7.54 & 7.68 & 7.66 & 7.53 & 7.56 & 7.47 & 7.48 \\
\hline $\mathrm{Al}_{2} \mathrm{O}_{3}$ & 6.27 & 6.49 & 6.52 & 6.61 & 6.70 & 6.68 & 6.63 & 6.66 & 6.71 & 6.74 \\
\hline $\mathrm{MgO}$ & 7.71 & 7.59 & 7.50 & 7.49 & 7.51 & 7.60 & 7.71 & 7.77 & 7.82 & 7.82 \\
\hline $\mathrm{FeO}$ & 3.72 & 3.76 & 3.74 & 3.91 & 3.90 & 3.87 & 3.81 & 3.78 & 3.87 & 3.87 \\
\hline $\mathrm{CaO}$ & 6.15 & 6.06 & 5.97 & 5.90 & 5.87 & 5.92 & 6.07 & 6.07 & 6.12 & 6.05 \\
\hline $\mathrm{Na}_{2} \mathrm{O}$ & 1.71 & 1.73 & 1.73 & 1.71 & 1.73 & 1.74 & 1.71 & 1.71 & 1.70 & 1.69 \\
\hline $\mathrm{K}_{2} \mathrm{O}$ & 0.73 & 0.69 & 0.68 & 0.65 & 0.63 & 0.63 & 0.63 & 0.63 & 0.64 & 0.66 \\
\hline$N$ & 222 & 235 & 240 & 249 & 263 & 273 & 290 & 284 & 305 & 320 \\
\hline
\end{tabular}


Table A.3: Mean and standard deviation of major element oxides as a function of $V_{p}$ for a warm continental geotherm $\left(q_{s}=90 \mathrm{mWm}^{-2}\right) . N$ denotes number of synthetic compositions in each $V_{p}$ bin.

\begin{tabular}{|c|c|c|c|c|c|c|c|c|c|c|}
\hline Depth $(\mathrm{km})$ & 5 & 10 & 15 & 20 & 25 & 30 & 35 & 40 & 45 & 50 \\
\hline $\mathrm{T}(\mathrm{C})$ & 135.97 & 237.53 & 327.32 & 409.97 & 488.29 & 563.98 & 638.08 & 711.21 & 783.76 & 855.95 \\
\hline$P$ (kbar) & 1.62 & 3.23 & 4.85 & 6.47 & 8.09 & 9.70 & 11.32 & 12.94 & 14.55 & 16.17 \\
\hline \multicolumn{11}{|c|}{$V_{p}=6.2 \mathrm{~km} / \mathrm{s}$} \\
\hline Mean & & & & & & & & & & \\
\hline $\mathrm{SiO}_{2}$ & 75.09 & 74.18 & 74.33 & 74.29 & 74.49 & 74.96 & 74.67 & 74.75 & 75.21 & 75.46 \\
\hline $\mathrm{Al}_{2} \mathrm{O}_{3}$ & 7.80 & 8.54 & 8.47 & 8.37 & 8.26 & 7.97 & 8.17 & 8.11 & 8.03 & 7.82 \\
\hline $\mathrm{MgO}$ & 2.30 & 2.41 & 2.39 & 2.41 & 2.38 & 2.35 & 2.18 & 2.20 & 2.23 & 2.09 \\
\hline $\mathrm{FeO}$ & 7.34 & 7.17 & 7.17 & 7.31 & 7.30 & 7.33 & 7.69 & 7.67 & 7.25 & 7.34 \\
\hline $\mathrm{CaO}$ & 2.18 & 2.18 & 2.17 & 2.20 & 2.18 & 2.10 & 2.04 & 2.03 & 1.92 & 1.97 \\
\hline $\mathrm{Na}_{2} \mathrm{O}$ & 2.38 & 2.58 & 2.55 & 2.53 & 2.49 & 2.41 & 2.45 & 2.44 & 2.46 & 2.41 \\
\hline $\mathrm{K}_{2} \mathrm{O}$ & 2.91 & 2.94 & 2.93 & 2.92 & 2.90 & 2.89 & 2.80 & 2.81 & 2.92 & 2.92 \\
\hline \multicolumn{11}{|l|}{ Std. Dev. } \\
\hline $\mathrm{SiO}_{2}$ & 6.52 & 7.46 & 7.54 & 7.49 & 7.60 & 7.74 & 7.78 & 7.79 & 7.72 & 7.81 \\
\hline $\mathrm{Al}_{2} \mathrm{O}_{3}$ & 5.31 & 6.22 & 6.23 & 6.23 & 6.24 & 6.21 & 6.27 & 6.27 & 6.34 & 6.40 \\
\hline $\mathrm{MgO}$ & 1.40 & 1.60 & 1.60 & 1.59 & 1.59 & 1.60 & 1.44 & 1.44 & 1.43 & 1.14 \\
\hline $\mathrm{FeO}$ & 5.89 & 5.78 & 5.75 & 5.80 & 5.76 & 5.73 & 5.70 & 5.71 & 5.51 & 5.53 \\
\hline $\mathrm{CaO}$ & 1.30 & 1.33 & 1.33 & 1.34 & 1.34 & 1.32 & 1.24 & 1.23 & 1.13 & 1.12 \\
\hline $\mathrm{Na}_{2} \mathrm{O}$ & 1.35 & 1.60 & 1.61 & 1.61 & 1.62 & 1.63 & 1.65 & 1.65 & 1.68 & 1.70 \\
\hline $\mathrm{K}_{2} \mathrm{O}$ & 1.41 & 1.38 & 1.38 & 1.37 & 1.36 & 1.37 & 1.34 & 1.34 & 1.29 & 1.30 \\
\hline$N$ & 171 & 181 & 183 & 186 & 189 & 190 & 179 & 177 & 164 & 156 \\
\hline \multicolumn{11}{|c|}{$V_{p}=6.3 \mathrm{~km} / \mathrm{s}$} \\
\hline Mean & & & & & & & & & & \\
\hline $\mathrm{SiO}_{2}$ & 71.88 & 71.36 & 71.15 & 71.40 & 71.51 & 71.69 & 71.70 & 71.81 & 71.84 & 71.82 \\
\hline $\mathrm{Al}_{2} \mathrm{O}_{3}$ & 9.93 & 10.35 & 10.46 & 10.30 & 10.24 & 10.13 & 10.11 & 10.00 & 10.01 & 10.07 \\
\hline $\mathrm{MgO}$ & 3.19 & 3.26 & 3.27 & 3.26 & 3.26 & 3.18 & 3.18 & 3.15 & 3.15 & 3.14 \\
\hline $\mathrm{FeO}$ & 6.97 & 6.87 & 6.91 & 6.82 & 6.74 & 6.72 & 6.73 & 6.83 & 6.80 & 6.79 \\
\hline $\mathrm{CaO}$ & 2.60 & 2.56 & 2.61 & 2.58 & 2.57 & 2.58 & 2.57 & 2.55 & 2.55 & 2.48 \\
\hline $\mathrm{Na}_{2} \mathrm{O}$ & 2.72 & 2.85 & 2.86 & 2.85 & 2.85 & 2.85 & 2.85 & 2.81 & 2.81 & 2.85 \\
\hline $\mathrm{K}_{2} \mathrm{O}$ & 2.72 & 2.76 & 2.74 & 2.80 & 2.83 & 2.87 & 2.87 & 2.84 & 2.85 & 2.87 \\
\hline \multicolumn{11}{|l|}{ Std. Dev. } \\
\hline $\mathrm{SiO}_{2}$ & 8.51 & 9.08 & 9.09 & 9.32 & 9.34 & 9.46 & 9.44 & 9.28 & 9.28 & 9.17 \\
\hline $\mathrm{Al}_{2} \mathrm{O}_{3}$ & 7.55 & 7.96 & 7.92 & 7.99 & 7.96 & 8.03 & 8.03 & 7.92 & 7.94 & 7.89 \\
\hline $\mathrm{MgO}$ & 2.05 & 2.06 & 2.03 & 1.99 & 1.98 & 2.00 & 1.99 & 2.00 & 2.00 & 1.98 \\
\hline $\mathrm{FeO}$ & 5.17 & 5.14 & 5.07 & 5.04 & 5.02 & 4.99 & 4.99 & 5.06 & 5.04 & 5.03 \\
\hline $\mathrm{CaO}$ & 1.60 & 1.60 & 1.63 & 1.61 & 1.58 & 1.57 & 1.57 & 1.57 & 1.57 & 1.55 \\
\hline $\mathrm{Na}_{2} \mathrm{O}$ & 1.92 & 2.06 & 2.05 & 2.05 & 2.04 & 2.05 & 2.05 & 2.03 & 2.03 & 2.01 \\
\hline $\mathrm{K}_{2} \mathrm{O}$ & 1.22 & 1.22 & 1.20 & 1.23 & 1.25 & 1.28 & 1.28 & 1.30 & 1.29 & 1.29 \\
\hline$N$ & 263 & 271 & 279 & 289 & 298 & 302 & 303 & 307 & 306 & 311 \\
\hline
\end{tabular}


Table A.3: continued

\begin{tabular}{|c|c|c|c|c|c|c|c|c|c|c|}
\hline Depth $(\mathrm{km})$ & 5 & 10 & 15 & 20 & 25 & 30 & 35 & 40 & 45 & 50 \\
\hline $\mathrm{T}(\mathrm{C})$ & 135.97 & 237.53 & 327.32 & 409.97 & 488.29 & 563.98 & 638.08 & 711.21 & 783.76 & 855.95 \\
\hline $\mathrm{P}$ (kbar) & 1.62 & 3.23 & 4.85 & 6.47 & 8.09 & 9.70 & 11.32 & 12.94 & 14.55 & 16.17 \\
\hline \multicolumn{11}{|c|}{$V_{p}=6.4 \mathrm{~km} / \mathrm{s}$} \\
\hline Mean & & & & & & & & & & \\
\hline $\mathrm{SiO}_{2}$ & 66.75 & 66.23 & 65.88 & 65.76 & 65.73 & 65.72 & 66.16 & 66.15 & 65.98 & 66.07 \\
\hline $\mathrm{Al}_{2} \mathrm{O}_{3}$ & 13.03 & 13.14 & 13.46 & 13.56 & 13.58 & 13.61 & 13.32 & 13.31 & 13.21 & 13.20 \\
\hline $\mathrm{MgO}$ & 4.28 & 4.34 & 4.33 & 4.36 & 4.41 & 4.38 & 4.40 & 4.39 & 4.27 & 4.29 \\
\hline $\mathrm{FeO}$ & 6.81 & 7.25 & 7.26 & 7.25 & 7.21 & 7.19 & 7.01 & 7.08 & 7.57 & 7.50 \\
\hline $\mathrm{CaO}$ & 3.18 & 3.19 & 3.17 & 3.13 & 3.12 & 3.13 & 3.13 & 3.12 & 3.08 & 3.02 \\
\hline $\mathrm{Na}_{2} \mathrm{O}$ & 3.37 & 3.37 & 3.44 & 3.47 & 3.48 & 3.49 & 3.43 & 3.43 & 3.42 & 3.43 \\
\hline $\mathrm{K}_{2} \mathrm{O}$ & 2.60 & 2.51 & 2.48 & 2.48 & 2.50 & 2.50 & 2.56 & 2.55 & 2.49 & 2.51 \\
\hline \multicolumn{11}{|l|}{ Std. Dev. } \\
\hline $\mathrm{SiO}_{2}$ & 9.34 & 9.73 & 9.89 & 10.04 & 9.99 & 9.95 & 10.13 & 10.20 & 10.59 & 10.53 \\
\hline $\mathrm{Al}_{2} \mathrm{O}_{3}$ & 8.74 & 8.68 & 8.85 & 8.87 & 8.82 & 8.78 & 8.81 & 8.80 & 8.86 & 8.79 \\
\hline $\mathrm{MgO}$ & 2.79 & 2.77 & 2.74 & 2.76 & 2.79 & 2.79 & 2.77 & 2.78 & 2.80 & 2.80 \\
\hline $\mathrm{FeO}$ & 4.97 & 5.37 & 5.40 & 5.37 & 5.34 & 5.32 & 5.34 & 5.36 & 5.65 & 5.63 \\
\hline $\mathrm{CaO}$ & 2.07 & 2.04 & 2.03 & 2.03 & 2.01 & 2.01 & 2.00 & 2.01 & 1.96 & 1.97 \\
\hline $\mathrm{Na}_{2} \mathrm{O}$ & 2.20 & 2.18 & 2.22 & 2.24 & 2.23 & 2.22 & 2.21 & 2.21 & 2.25 & 2.23 \\
\hline $\mathrm{K}_{2} \mathrm{O}$ & 1.06 & 1.11 & 1.11 & 1.11 & 1.11 & 1.11 & 1.15 & 1.15 & 1.19 & 1.19 \\
\hline$N$ & 309 & 317 & 330 & 328 & 332 & 335 & 346 & 347 & 358 & 364 \\
\hline \multicolumn{11}{|c|}{$V_{p}=6.5 \mathrm{~km} / \mathrm{s}$} \\
\hline Mean & & & & & & & & & & \\
\hline $\mathrm{SiO}_{2}$ & 60.33 & 60.57 & 60.11 & 60.17 & 59.91 & 59.83 & 59.66 & 59.75 & 59.77 & 59.69 \\
\hline $\mathrm{Al}_{2} \mathrm{O}_{3}$ & 16.91 & 16.58 & 16.82 & 16.73 & 16.88 & 16.88 & 16.96 & 16.81 & 16.79 & 16.82 \\
\hline $\mathrm{MgO}$ & 4.78 & 4.95 & 5.06 & 5.06 & 5.07 & 5.08 & 5.07 & 5.16 & 5.15 & 5.13 \\
\hline $\mathrm{FeO}$ & 7.67 & 7.66 & 7.76 & 7.83 & 7.95 & 8.07 & 8.16 & 8.22 & 8.24 & 8.32 \\
\hline $\mathrm{CaO}$ & 4.19 & 4.24 & 4.20 & 4.20 & 4.18 & 4.14 & 4.08 & 3.98 & 3.98 & 3.97 \\
\hline $\mathrm{Na}_{2} \mathrm{O}$ & 4.01 & 3.91 & 3.98 & 3.95 & 3.98 & 4.00 & 4.04 & 4.03 & 4.03 & 4.04 \\
\hline $\mathrm{K}_{2} \mathrm{O}$ & 2.12 & 2.10 & 2.09 & 2.07 & 2.04 & 2.04 & 2.05 & 2.07 & 2.06 & 2.05 \\
\hline \multicolumn{11}{|l|}{ Std. Dev. } \\
\hline $\mathrm{SiO}_{2}$ & 9.64 & 9.43 & 9.67 & 9.64 & 9.80 & 9.99 & 10.09 & 10.05 & 10.05 & 10.28 \\
\hline $\mathrm{Al}_{2} \mathrm{O}_{3}$ & 8.29 & 8.17 & 8.27 & 8.27 & 8.37 & 8.39 & 8.48 & 8.37 & 8.38 & 8.41 \\
\hline $\mathrm{MgO}$ & 3.10 & 3.28 & 3.34 & 3.35 & 3.35 & 3.31 & 3.32 & 3.34 & 3.34 & 3.33 \\
\hline $\mathrm{FeO}$ & 5.72 & 5.67 & 5.65 & 5.72 & 5.72 & 5.75 & 5.75 & 5.77 & 5.78 & 5.87 \\
\hline $\mathrm{CaO}$ & 2.77 & 2.76 & 2.78 & 2.77 & 2.75 & 2.73 & 2.71 & 2.66 & 2.66 & 2.65 \\
\hline $\mathrm{Na}_{2} \mathrm{O}$ & 2.18 & 2.12 & 2.16 & 2.16 & 2.17 & 2.18 & 2.20 & 2.18 & 2.19 & 2.20 \\
\hline $\mathrm{K}_{2} \mathrm{O}$ & 1.10 & 1.08 & 1.08 & 1.09 & 1.09 & 1.09 & 1.10 & 1.10 & 1.10 & 1.11 \\
\hline$N$ & 406 & 417 & 429 & 432 & 442 & 452 & 446 & 440 & 440 & 446 \\
\hline
\end{tabular}


Table A.3: continued

\begin{tabular}{|c|c|c|c|c|c|c|c|c|c|c|}
\hline Depth $(\mathrm{km})$ & 5 & 10 & 15 & 20 & 25 & 30 & 35 & 40 & 45 & 50 \\
\hline $\mathrm{T}(\mathrm{C})$ & 135.97 & 237.53 & 327.32 & 409.97 & 488.29 & 563.98 & 638.08 & 711.21 & 783.76 & 855.95 \\
\hline $\mathrm{P}$ (kbar) & 1.62 & 3.23 & 4.85 & 6.47 & 8.09 & 9.70 & 11.32 & 12.94 & 14.55 & 16.17 \\
\hline \multicolumn{11}{|c|}{$V_{p}=6.6 \mathrm{~km} / \mathrm{s}$} \\
\hline Mean & & & & & & & & & & \\
\hline $\mathrm{SiO}_{2}$ & 57.94 & 57.38 & 57.20 & 57.11 & 57.09 & 57.26 & 57.31 & 57.24 & 57.45 & 57.42 \\
\hline $\mathrm{Al}_{2} \mathrm{O}_{3}$ & 17.76 & 18.08 & 18.04 & 18.03 & 17.92 & 17.71 & 17.64 & 17.65 & 17.55 & 17.52 \\
\hline $\mathrm{MgO}$ & 5.44 & 5.53 & 5.58 & 5.64 & 5.70 & 5.84 & 5.89 & 5.96 & 6.00 & 6.01 \\
\hline $\mathrm{FeO}$ & 8.20 & 8.31 & 8.47 & 8.53 & 8.62 & 8.55 & 8.60 & 8.62 & 8.48 & 8.56 \\
\hline $\mathrm{CaO}$ & 4.89 & 4.89 & 4.96 & 4.93 & 4.97 & 4.93 & 4.89 & 4.83 & 4.84 & 4.81 \\
\hline $\mathrm{Na}_{2} \mathrm{O}$ & 4.00 & 4.06 & 4.05 & 4.05 & 4.02 & 3.98 & 3.97 & 3.99 & 3.95 & 3.96 \\
\hline $\mathrm{K}_{2} \mathrm{O}$ & 1.79 & 1.75 & 1.72 & 1.72 & 1.70 & 1.72 & 1.72 & 1.73 & 1.73 & 1.73 \\
\hline \multicolumn{11}{|l|}{ Std. Dev. } \\
\hline $\mathrm{SiO}_{2}$ & 9.79 & 9.89 & 9.95 & 10.05 & 10.06 & 10.17 & 10.18 & 10.20 & 10.04 & 10.09 \\
\hline $\mathrm{Al}_{2} \mathrm{O}_{3}$ & 8.18 & 8.25 & 8.25 & 8.27 & 8.26 & 8.37 & 8.39 & 8.37 & 8.33 & 8.34 \\
\hline $\mathrm{MgO}$ & 3.45 & 3.44 & 3.42 & 3.43 & 3.40 & 3.52 & 3.52 & 3.56 & 3.56 & 3.57 \\
\hline $\mathrm{FeO}$ & 5.61 & 5.60 & 5.58 & 5.62 & 5.64 & 5.65 & 5.67 & 5.70 & 5.66 & 5.68 \\
\hline $\mathrm{CaO}$ & 2.96 & 3.01 & 3.02 & 3.01 & 3.06 & 3.03 & 3.01 & 3.01 & 3.00 & 2.99 \\
\hline $\mathrm{Na}_{2} \mathrm{O}$ & 2.23 & 2.26 & 2.26 & 2.28 & 2.28 & 2.30 & 2.31 & 2.30 & 2.27 & 2.28 \\
\hline $\mathrm{K}_{2} \mathrm{O}$ & 1.05 & 1.06 & 1.06 & 1.06 & 1.04 & 1.04 & 1.04 & 1.04 & 1.04 & 1.04 \\
\hline$N$ & 514 & 539 & 549 & 559 & 575 & 586 & 586 & 594 & 603 & 608 \\
\hline \multicolumn{11}{|c|}{$V_{p}=6.7 \mathrm{~km} / \mathrm{s}$} \\
\hline Mean & & & & & & & & & & \\
\hline $\mathrm{SiO}_{2}$ & 56.63 & 56.18 & 56.13 & 56.00 & 56.04 & 56.15 & 56.25 & 56.24 & 56.21 & 56.26 \\
\hline $\mathrm{Al}_{2} \mathrm{O}_{3}$ & 17.51 & 17.67 & 17.63 & 17.69 & 17.63 & 17.55 & 17.48 & 17.51 & 17.48 & 17.44 \\
\hline $\mathrm{MgO}$ & 6.91 & 7.14 & 7.23 & 7.33 & 7.38 & 7.43 & 7.47 & 7.44 & 7.52 & 7.59 \\
\hline $\mathrm{FeO}$ & 8.22 & 8.35 & 8.34 & 8.30 & 8.25 & 8.18 & 8.17 & 8.16 & 8.16 & 8.09 \\
\hline $\mathrm{CaO}$ & 5.43 & 5.40 & 5.45 & 5.41 & 5.43 & 5.45 & 5.42 & 5.42 & 5.38 & 5.37 \\
\hline $\mathrm{Na}_{2} \mathrm{O}$ & 3.75 & 3.77 & 3.75 & 3.78 & 3.77 & 3.74 & 3.72 & 3.73 & 3.74 & 3.72 \\
\hline $\mathrm{K}_{2} \mathrm{O}$ & 1.55 & 1.49 & 1.48 & 1.50 & 1.51 & 1.52 & 1.51 & 1.51 & 1.52 & 1.53 \\
\hline \multicolumn{11}{|l|}{ Std. Dev. } \\
\hline $\mathrm{SiO}_{2}$ & 9.93 & 10.05 & 10.04 & 10.06 & 10.06 & 10.00 & 9.98 & 9.99 & 9.99 & 9.92 \\
\hline $\mathrm{Al}_{2} \mathrm{O}_{3}$ & 8.42 & 8.46 & 8.44 & 8.47 & 8.45 & 8.40 & 8.32 & 8.39 & 8.39 & 8.37 \\
\hline $\mathrm{MgO}$ & 3.63 & 3.68 & 3.66 & 3.68 & 3.67 & 3.72 & 3.74 & 3.87 & 3.94 & 3.97 \\
\hline $\mathrm{FeO}$ & 5.30 & 5.43 & 5.45 & 5.39 & 5.38 & 5.34 & 5.33 & 5.28 & 5.30 & 5.22 \\
\hline $\mathrm{CaO}$ & 3.43 & 3.42 & 3.44 & 3.42 & 3.42 & 3.42 & 3.40 & 3.41 & 3.40 & 3.40 \\
\hline $\mathrm{Na}_{2} \mathrm{O}$ & 2.34 & 2.38 & 2.36 & 2.37 & 2.38 & 2.36 & 2.35 & 2.36 & 2.35 & 2.34 \\
\hline $\mathrm{K}_{2} \mathrm{O}$ & 0.96 & 0.96 & 0.96 & 0.96 & 0.96 & 0.97 & 0.96 & 0.96 & 0.96 & 0.96 \\
\hline$N$ & 603 & 642 & 651 & 665 & 656 & 659 & 669 & 677 & 682 & 684 \\
\hline
\end{tabular}


Table A.3: continued

\begin{tabular}{|c|c|c|c|c|c|c|c|c|c|c|}
\hline Depth $(\mathrm{km})$ & 5 & 10 & 15 & 20 & 25 & 30 & 35 & 40 & 45 & 50 \\
\hline $\mathrm{T}(\mathrm{C})$ & 135.97 & 237.53 & 327.32 & 409.97 & 488.29 & 563.98 & 638.08 & 711.21 & 783.76 & 855.95 \\
\hline $\mathrm{P}(\mathrm{kbar})$ & 1.62 & 3.23 & 4.85 & 6.47 & 8.09 & 9.70 & 11.32 & 12.94 & 14.55 & 16.17 \\
\hline \multicolumn{11}{|c|}{$V_{p}=6.8 \mathrm{~km} / \mathrm{s}$} \\
\hline Mean & & & & & & & & & & \\
\hline $\mathrm{SiO}_{2}$ & 54.98 & 55.37 & 55.29 & 55.40 & 55.50 & 55.35 & 55.25 & 55.34 & 55.25 & 55.42 \\
\hline $\mathrm{Al}_{2} \mathrm{O}_{3}$ & 17.90 & 17.50 & 17.39 & 17.31 & 17.19 & 17.31 & 17.32 & 17.27 & 17.30 & 17.10 \\
\hline $\mathrm{MgO}$ & 8.24 & 8.61 & 8.86 & 8.95 & 9.07 & 9.02 & 9.06 & 9.02 & 9.07 & 9.16 \\
\hline $\mathrm{FeO}$ & 7.68 & 7.24 & 7.23 & 7.12 & 7.09 & 7.16 & 7.23 & 7.16 & 7.18 & 7.10 \\
\hline $\mathrm{CaO}$ & 6.04 & 6.19 & 6.17 & 6.20 & 6.16 & 6.18 & 6.15 & 6.24 & 6.23 & 6.29 \\
\hline $\mathrm{Na}_{2} \mathrm{O}$ & 3.70 & 3.58 & 3.56 & 3.53 & 3.49 & 3.51 & 3.53 & 3.50 & 3.50 & 3.45 \\
\hline $\mathrm{K}_{2} \mathrm{O}$ & 1.47 & 1.52 & 1.51 & 1.52 & 1.51 & 1.49 & 1.48 & 1.49 & 1.48 & 1.49 \\
\hline \multicolumn{11}{|l|}{ Std. Dev. } \\
\hline $\mathrm{SiO}_{2}$ & 9.62 & 9.51 & 9.41 & 9.25 & 9.21 & 9.09 & 9.06 & 9.03 & 9.00 & 8.91 \\
\hline $\mathrm{Al}_{2} \mathrm{O}_{3}$ & 8.33 & 8.27 & 8.19 & 8.14 & 8.20 & 8.09 & 8.04 & 8.06 & 8.03 & 8.06 \\
\hline $\mathrm{MgO}$ & 4.35 & 4.54 & 4.57 & 4.65 & 4.72 & 4.71 & 4.75 & 4.79 & 4.81 & 4.83 \\
\hline $\mathrm{FeO}$ & 5.22 & 5.03 & 4.98 & 4.90 & 4.87 & 4.86 & 4.90 & 4.81 & 4.80 & 4.74 \\
\hline $\mathrm{CaO}$ & 3.96 & 3.92 & 3.97 & 3.95 & 3.93 & 3.91 & 3.90 & 3.91 & 3.91 & 3.89 \\
\hline $\mathrm{Na}_{2} \mathrm{O}$ & 2.31 & 2.27 & 2.24 & 2.21 & 2.21 & 2.20 & 2.19 & 2.20 & 2.19 & 2.19 \\
\hline $\mathrm{K}_{2} \mathrm{O}$ & 0.92 & 0.92 & 0.90 & 0.89 & 0.89 & 0.90 & 0.90 & 0.89 & 0.89 & 0.89 \\
\hline$N$ & 690 & 685 & 704 & 709 & 706 & 700 & 709 & 711 & 711 & 723 \\
\hline \multicolumn{11}{|c|}{$V_{p}=6.9 \mathrm{~km} / \mathrm{s}$} \\
\hline Mean & & & & & & & & & & \\
\hline $\mathrm{SiO}_{2}$ & 53.64 & 53.71 & 53.93 & 53.92 & 53.87 & 53.84 & 53.71 & 53.73 & 53.66 & 53.71 \\
\hline $\mathrm{Al}_{2} \mathrm{O}_{3}$ & 17.92 & 17.82 & 17.54 & 17.43 & 17.43 & 17.43 & 17.49 & 17.47 & 17.47 & 17.33 \\
\hline $\mathrm{MgO}$ & 9.74 & 9.83 & 9.92 & 10.07 & 10.19 & 10.19 & 10.18 & 10.21 & 10.22 & 10.29 \\
\hline $\mathrm{FeO}$ & 7.11 & 6.87 & 6.75 & 6.77 & 6.79 & 6.81 & 6.85 & 6.81 & 6.87 & 6.94 \\
\hline $\mathrm{CaO}$ & 6.59 & 6.79 & 7.02 & 7.03 & 6.96 & 6.98 & 7.01 & 7.05 & 7.04 & 7.02 \\
\hline $\mathrm{Na}_{2} \mathrm{O}$ & 3.60 & 3.54 & 3.43 & 3.39 & 3.39 & 3.38 & 3.39 & 3.38 & 3.38 & 3.36 \\
\hline $\mathrm{K}_{2} \mathrm{O}$ & 1.43 & 1.45 & 1.43 & 1.40 & 1.39 & 1.38 & 1.38 & 1.38 & 1.37 & 1.36 \\
\hline \multicolumn{11}{|l|}{ Std. Dev. } \\
\hline $\mathrm{SiO}_{2}$ & 9.10 & 9.00 & 8.88 & 8.78 & 8.74 & 8.76 & 8.81 & 8.79 & 8.79 & 8.78 \\
\hline $\mathrm{Al}_{2} \mathrm{O}_{3}$ & 8.13 & 8.09 & 8.03 & 7.96 & 7.90 & 7.91 & 7.94 & 7.96 & 7.93 & 7.99 \\
\hline $\mathrm{MgO}$ & 5.20 & 5.35 & 5.42 & 5.51 & 5.55 & 5.55 & 5.57 & 5.54 & 5.57 & 5.58 \\
\hline $\mathrm{FeO}$ & 4.82 & 4.60 & 4.53 & 4.60 & 4.63 & 4.62 & 4.67 & 4.71 & 4.72 & 4.74 \\
\hline $\mathrm{CaO}$ & 4.34 & 4.34 & 4.50 & 4.49 & 4.50 & 4.50 & 4.48 & 4.44 & 4.43 & 4.39 \\
\hline $\mathrm{Na}_{2} \mathrm{O}$ & 2.16 & 2.14 & 2.13 & 2.10 & 2.07 & 2.07 & 2.08 & 2.08 & 2.08 & 2.08 \\
\hline $\mathrm{K}_{2} \mathrm{O}$ & 0.90 & 0.89 & 0.87 & 0.87 & 0.86 & 0.86 & 0.85 & 0.85 & 0.84 & 0.85 \\
\hline$N$ & 678 & 645 & 650 & 651 & 669 & 669 & 675 & 683 & 689 & 695 \\
\hline
\end{tabular}


Table A.3: continued

\begin{tabular}{|c|c|c|c|c|c|c|c|c|c|c|}
\hline Depth $(\mathrm{km})$ & 5 & 10 & 15 & 20 & 25 & 30 & 35 & 40 & 45 & 50 \\
\hline $\mathrm{T}(\mathrm{C})$ & 135.97 & 237.53 & 327.32 & 409.97 & 488.29 & 563.98 & 638.08 & 711.21 & 783.76 & 855.95 \\
\hline $\mathrm{P}$ (kbar) & 1.62 & 3.23 & 4.85 & 6.47 & 8.09 & 9.70 & 11.32 & 12.94 & 14.55 & 16.17 \\
\hline \multicolumn{11}{|c|}{$V_{p}=7.0 \mathrm{~km} / \mathrm{s}$} \\
\hline Mean & & & & & & & & & & \\
\hline $\mathrm{SiO}_{2}$ & 52.60 & 52.32 & 52.41 & 52.22 & 51.74 & 51.68 & 51.50 & 51.28 & 51.33 & 51.17 \\
\hline $\mathrm{Al}_{2} \mathrm{O}_{3}$ & 17.59 & 17.66 & 17.54 & 17.57 & 17.85 & 17.83 & 17.97 & 18.05 & 17.89 & 17.94 \\
\hline $\mathrm{MgO}$ & 11.08 & 11.21 & 11.31 & 11.41 & 11.45 & 11.70 & 11.72 & 11.84 & 12.06 & 12.10 \\
\hline $\mathrm{FeO}$ & 7.08 & 7.25 & 7.17 & 7.26 & 7.26 & 7.11 & 7.09 & 7.18 & 7.15 & 7.25 \\
\hline $\mathrm{CaO}$ & 7.09 & 7.07 & 7.12 & 7.12 & 7.21 & 7.21 & 7.21 & 7.13 & 7.11 & 7.07 \\
\hline $\mathrm{Na}_{2} \mathrm{O}$ & 3.35 & 3.35 & 3.30 & 3.31 & 3.37 & 3.35 & 3.39 & 3.41 & 3.37 & 3.39 \\
\hline $\mathrm{K}_{2} \mathrm{O}$ & 1.22 & 1.16 & 1.15 & 1.13 & 1.13 & 1.13 & 1.13 & 1.11 & 1.11 & 1.09 \\
\hline \multicolumn{11}{|l|}{ Std. Dev. } \\
\hline $\mathrm{SiO}_{2}$ & 8.92 & 8.81 & 8.81 & 8.75 & 8.79 & 8.74 & 8.87 & 8.89 & 8.97 & 8.90 \\
\hline $\mathrm{Al}_{2} \mathrm{O}_{3}$ & 7.89 & 7.83 & 7.87 & 7.87 & 7.84 & 7.86 & 7.92 & 7.95 & 8.02 & 8.01 \\
\hline $\mathrm{MgO}$ & 5.35 & 5.42 & 5.55 & 5.64 & 5.72 & 5.84 & 5.90 & 5.91 & 5.95 & 6.00 \\
\hline $\mathrm{FeO}$ & 4.78 & 4.84 & 4.83 & 4.83 & 4.93 & 4.95 & 4.96 & 4.99 & 4.91 & 4.93 \\
\hline $\mathrm{CaO}$ & 4.66 & 4.68 & 4.68 & 4.67 & 4.73 & 4.74 & 4.76 & 4.76 & 4.73 & 4.74 \\
\hline $\mathrm{Na}_{2} \mathrm{O}$ & 2.10 & 2.08 & 2.07 & 2.07 & 2.08 & 2.07 & 2.10 & 2.09 & 2.11 & 2.12 \\
\hline $\mathrm{K}_{2} \mathrm{O}$ & 0.82 & 0.78 & 0.80 & 0.80 & 0.80 & 0.78 & 0.78 & 0.79 & 0.78 & 0.77 \\
\hline$N$ & 596 & 587 & 573 & 579 & 585 & 613 & 616 & 618 & 641 & 640 \\
\hline \multicolumn{11}{|c|}{$V_{p}=7.1 \mathrm{~km} / \mathrm{s}$} \\
\hline Mean & & & & & & & & & & \\
\hline $\mathrm{SiO}_{2}$ & 51.42 & 51.16 & 50.64 & 50.47 & 50.47 & 50.45 & 50.55 & 50.28 & 50.26 & 50.05 \\
\hline $\mathrm{Al}_{2} \mathrm{O}_{3}$ & 17.37 & 17.36 & 17.75 & 17.93 & 17.88 & 17.92 & 17.89 & 18.07 & 18.16 & 18.27 \\
\hline $\mathrm{MgO}$ & 12.37 & 12.55 & 12.68 & 12.60 & 12.56 & 12.57 & 12.56 & 12.60 & 12.54 & 12.60 \\
\hline $\mathrm{FeO}$ & 7.06 & 7.23 & 7.26 & 7.19 & 7.15 & 7.12 & 7.02 & 7.11 & 7.02 & 6.99 \\
\hline $\mathrm{CaO}$ & 7.55 & 7.51 & 7.37 & 7.49 & 7.64 & 7.64 & 7.71 & 7.66 & 7.75 & 7.84 \\
\hline $\mathrm{Na}_{2} \mathrm{O}$ & 3.17 & 3.18 & 3.28 & 3.31 & 3.28 & 3.28 & 3.25 & 3.30 & 3.30 & 3.30 \\
\hline $\mathrm{K}_{2} \mathrm{O}$ & 1.07 & 1.04 & 1.02 & 1.03 & 1.03 & 1.02 & 1.02 & 1.00 & 0.99 & 0.97 \\
\hline \multicolumn{11}{|l|}{ Std. Dev. } \\
\hline $\mathrm{SiO}_{2}$ & 8.57 & 8.33 & 8.33 & 8.43 & 8.39 & 8.41 & 8.40 & 8.45 & 8.49 & 8.51 \\
\hline $\mathrm{Al}_{2} \mathrm{O}_{3}$ & 7.54 & 7.53 & 7.55 & 7.57 & 7.58 & 7.59 & 7.63 & 7.69 & 7.73 & 7.75 \\
\hline $\mathrm{MgO}$ & 5.91 & 5.97 & 6.05 & 6.09 & 6.11 & 6.15 & 6.14 & 6.25 & 6.26 & 6.30 \\
\hline $\mathrm{FeO}$ & 5.03 & 4.98 & 5.02 & 4.95 & 4.95 & 4.89 & 4.84 & 4.81 & 4.80 & 4.74 \\
\hline $\mathrm{CaO}$ & 5.11 & 5.13 & 5.10 & 5.15 & 5.13 & 5.12 & 5.12 & 5.14 & 5.13 & 5.09 \\
\hline $\mathrm{Na}_{2} \mathrm{O}$ & 2.03 & 2.01 & 1.99 & 2.01 & 2.01 & 2.01 & 2.00 & 2.01 & 2.01 & 2.03 \\
\hline $\mathrm{K}_{2} \mathrm{O}$ & 0.76 & 0.75 & 0.75 & 0.75 & 0.75 & 0.74 & 0.75 & 0.73 & 0.72 & 0.72 \\
\hline$N$ & 551 & 567 & 552 & 544 & 532 & 541 & 540 & 549 & 541 & 551 \\
\hline
\end{tabular}


Table A.3: continued

\begin{tabular}{|c|c|c|c|c|c|c|c|c|c|c|}
\hline Depth $(\mathrm{km})$ & 5 & 10 & 15 & 20 & 25 & 30 & 35 & 40 & 45 & 50 \\
\hline $\mathrm{T}(\mathrm{C})$ & 135.97 & 237.53 & 327.32 & 409.97 & 488.29 & 563.98 & 638.08 & 711.21 & 783.76 & 855.95 \\
\hline$P$ (kbar) & 1.62 & 3.23 & 4.85 & 6.47 & 8.09 & 9.70 & 11.32 & 12.94 & 14.55 & 16.17 \\
\hline \multicolumn{11}{|c|}{$V_{p}=7.2 \mathrm{~km} / \mathrm{s}$} \\
\hline Mean & & & & & & & & & & \\
\hline $\mathrm{SiO}_{2}$ & 49.74 & 49.61 & 49.33 & 49.09 & 48.96 & 48.81 & 49.04 & 48.98 & 48.65 & 48.60 \\
\hline $\mathrm{Al}_{2} \mathrm{O}_{3}$ & 18.11 & 18.10 & 18.21 & 18.48 & 18.66 & 18.82 & 18.74 & 18.91 & 19.28 & 19.29 \\
\hline $\mathrm{MgO}$ & 12.87 & 12.82 & 12.98 & 12.86 & 12.86 & 12.54 & 12.42 & 12.28 & 12.01 & 12.09 \\
\hline $\mathrm{FeO}$ & 7.09 & 7.04 & 6.91 & 6.80 & 6.65 & 6.77 & 6.63 & 6.48 & 6.41 & 6.38 \\
\hline $\mathrm{CaO}$ & 7.97 & 8.27 & 8.42 & 8.58 & 8.67 & 8.88 & 9.06 & 9.25 & 9.50 & 9.54 \\
\hline $\mathrm{Na}_{2} \mathrm{O}$ & 3.26 & 3.22 & 3.21 & 3.25 & 3.27 & 3.27 & 3.21 & 3.21 & 3.26 & 3.24 \\
\hline $\mathrm{K}_{2} \mathrm{O}$ & 0.97 & 0.96 & 0.95 & 0.95 & 0.94 & 0.91 & 0.91 & 0.91 & 0.89 & 0.87 \\
\hline \multicolumn{11}{|l|}{ Std. Dev. } \\
\hline $\mathrm{SiO}_{2}$ & 8.46 & 8.31 & 8.14 & 8.27 & 8.21 & 8.22 & 8.14 & 8.15 & 7.98 & 7.92 \\
\hline $\mathrm{Al}_{2} \mathrm{O}_{3}$ & 7.81 & 7.83 & 7.76 & 7.80 & 7.80 & 7.79 & 7.70 & 7.70 & 7.56 & 7.52 \\
\hline $\mathrm{MgO}$ & 6.57 & 6.73 & 6.78 & 6.77 & 6.77 & 6.79 & 6.77 & 6.77 & 6.86 & 6.93 \\
\hline $\mathrm{FeO}$ & 4.83 & 4.76 & 4.70 & 4.70 & 4.50 & 4.44 & 4.31 & 4.23 & 4.26 & 4.24 \\
\hline $\mathrm{CaO}$ & 5.44 & 5.55 & 5.52 & 5.59 & 5.54 & 5.61 & 5.53 & 5.51 & 5.54 & 5.46 \\
\hline $\mathrm{Na}_{2} \mathrm{O}$ & 1.96 & 1.98 & 1.98 & 1.99 & 1.97 & 1.98 & 1.94 & 1.91 & 1.88 & 1.90 \\
\hline $\mathrm{K}_{2} \mathrm{O}$ & 0.72 & 0.70 & 0.70 & 0.72 & 0.71 & 0.71 & 0.70 & 0.70 & 0.70 & 0.70 \\
\hline$N$ & 469 & 478 & 481 & 475 & 465 & 438 & 439 & 439 & 424 & 434 \\
\hline \multicolumn{11}{|c|}{$V_{p}=7.3 \mathrm{~km} / \mathrm{s}$} \\
\hline Mean & & & & & & & & & & \\
\hline $\mathrm{SiO}_{2}$ & 47.78 & 47.19 & 47.53 & 47.74 & 47.71 & 47.83 & 47.63 & 47.67 & 47.64 & 47.67 \\
\hline $\mathrm{Al}_{2} \mathrm{O}_{3}$ & 19.41 & 19.96 & 19.53 & 19.23 & 19.27 & 19.11 & 19.16 & 19.22 & 19.21 & 19.27 \\
\hline $\mathrm{MgO}$ & 12.95 & 12.95 & 13.04 & 13.17 & 13.20 & 13.33 & 13.56 & 13.33 & 13.39 & 13.29 \\
\hline $\mathrm{FeO}$ & 6.60 & 6.30 & 6.29 & 6.35 & 6.24 & 6.14 & 6.07 & 5.95 & 5.96 & 5.85 \\
\hline $\mathrm{CaO}$ & 9.02 & 9.30 & 9.46 & 9.45 & 9.53 & 9.60 & 9.56 & 9.86 & 9.82 & 9.94 \\
\hline $\mathrm{Na}_{2} \mathrm{O}$ & 3.37 & 3.44 & 3.31 & 3.23 & 3.23 & 3.17 & 3.19 & 3.15 & 3.15 & 3.15 \\
\hline $\mathrm{K}_{2} \mathrm{O}$ & 0.87 & 0.86 & 0.85 & 0.84 & 0.84 & 0.84 & 0.84 & 0.83 & 0.83 & 0.84 \\
\hline \multicolumn{11}{|l|}{ Std. Dev. } \\
\hline $\mathrm{SiO}_{2}$ & 7.86 & 7.78 & 7.74 & 7.66 & 7.64 & 7.54 & 7.54 & 7.46 & 7.44 & 7.31 \\
\hline $\mathrm{Al}_{2} \mathrm{O}_{3}$ & 7.69 & 7.49 & 7.57 & 7.65 & 7.62 & 7.58 & 7.50 & 7.37 & 7.33 & 7.15 \\
\hline $\mathrm{MgO}$ & 7.02 & 7.32 & 7.21 & 7.22 & 7.22 & 7.29 & 7.40 & 7.40 & 7.37 & 7.44 \\
\hline $\mathrm{FeO}$ & 4.18 & 4.09 & 4.14 & 4.18 & 4.13 & 4.14 & 4.09 & 4.08 & 4.03 & 4.06 \\
\hline $\mathrm{CaO}$ & 5.55 & 5.75 & 5.67 & 5.59 & 5.68 & 5.68 & 5.69 & 5.74 & 5.72 & 5.84 \\
\hline $\mathrm{Na}_{2} \mathrm{O}$ & 1.90 & 1.89 & 1.92 & 1.91 & 1.90 & 1.89 & 1.87 & 1.85 & 1.83 & 1.80 \\
\hline $\mathrm{K}_{2} \mathrm{O}$ & 0.72 & 0.71 & 0.72 & 0.71 & 0.71 & 0.70 & 0.70 & 0.70 & 0.70 & 0.70 \\
\hline$N$ & 383 & 355 & 360 & 363 & 371 & 367 & 368 & 365 & 369 & 355 \\
\hline
\end{tabular}


Table A.3: continued

\begin{tabular}{|c|c|c|c|c|c|c|c|c|c|c|}
\hline Depth $(\mathrm{km})$ & 5 & 10 & 15 & 20 & 25 & 30 & 35 & 40 & 45 & 50 \\
\hline $\mathrm{T}(\mathrm{C})$ & 135.97 & 237.53 & 327.32 & 409.97 & 488.29 & 563.98 & 638.08 & 711.21 & 783.76 & 855.95 \\
\hline $\mathrm{P}$ (kbar) & 1.62 & 3.23 & 4.85 & 6.47 & 8.09 & 9.70 & 11.32 & 12.94 & 14.55 & 16.17 \\
\hline \multicolumn{11}{|c|}{$V_{p}=7.4 \mathrm{~km} / \mathrm{s}$} \\
\hline Mean & & & & & & & & & & \\
\hline $\mathrm{SiO}_{2}$ & 47.83 & 47.54 & 47.28 & 47.37 & 47.60 & 47.23 & 47.11 & 47.16 & 47.25 & 46.86 \\
\hline $\mathrm{Al}_{2} \mathrm{O}_{3}$ & 18.69 & 18.95 & 19.26 & 18.98 & 18.79 & 18.82 & 18.82 & 18.71 & 18.65 & 18.99 \\
\hline $\mathrm{MgO}$ & 13.73 & 13.69 & 13.49 & 13.80 & 13.78 & 14.27 & 14.36 & 14.44 & 14.51 & 14.47 \\
\hline $\mathrm{FeO}$ & 5.94 & 6.05 & 5.99 & 5.98 & 5.91 & 5.93 & 5.91 & 5.83 & 5.67 & 5.55 \\
\hline $\mathrm{CaO}$ & 9.95 & 9.84 & 10.02 & 10.03 & 10.13 & 9.95 & 10.00 & 10.06 & 10.13 & 10.27 \\
\hline $\mathrm{Na}_{2} \mathrm{O}$ & 3.03 & 3.11 & 3.15 & 3.06 & 3.00 & 3.03 & 3.03 & 3.00 & 2.97 & 3.04 \\
\hline $\mathrm{K}_{2} \mathrm{O}$ & 0.85 & 0.84 & 0.82 & 0.80 & 0.80 & 0.79 & 0.79 & 0.81 & 0.82 & 0.84 \\
\hline \multicolumn{11}{|l|}{ Std. Dev. } \\
\hline $\mathrm{SiO}_{2}$ & 7.30 & 7.44 & 7.56 & 7.32 & 7.23 & 7.22 & 7.19 & 7.07 & 7.06 & 6.98 \\
\hline $\mathrm{Al}_{2} \mathrm{O}_{3}$ & 7.20 & 7.09 & 7.00 & 6.82 & 6.65 & 6.53 & 6.63 & 6.48 & 6.27 & 6.13 \\
\hline $\mathrm{MgO}$ & 7.41 & 7.38 & 7.44 & 7.64 & 7.76 & 7.93 & 7.92 & 7.98 & 7.88 & 7.92 \\
\hline $\mathrm{FeO}$ & 4.05 & 4.16 & 4.16 & 4.16 & 4.11 & 4.03 & 4.00 & 4.01 & 3.90 & 3.83 \\
\hline $\mathrm{CaO}$ & 5.90 & 5.98 & 6.07 & 6.05 & 6.16 & 6.15 & 6.19 & 6.21 & 6.16 & 6.29 \\
\hline $\mathrm{Na}_{2} \mathrm{O}$ & 1.88 & 1.88 & 1.89 & 1.85 & 1.81 & 1.79 & 1.80 & 1.79 & 1.75 & 1.72 \\
\hline $\mathrm{K}_{2} \mathrm{O}$ & 0.70 & 0.71 & 0.69 & 0.65 & 0.65 & 0.64 & 0.64 & 0.64 & 0.67 & 0.66 \\
\hline$N$ & 333 & 308 & 290 & 284 & 282 & 291 & 291 & 288 & 286 & 272 \\
\hline \multirow{2}{*}{\multicolumn{11}{|c|}{$V_{p}=7.5 \mathrm{~km} / \mathrm{s}$}} \\
\hline & & & & & & & & & & \\
\hline $\mathrm{SiO}_{2}$ & 47.37 & 47.43 & 46.56 & 46.02 & 45.52 & 45.03 & 44.87 & 44.86 & 44.99 & 44.76 \\
\hline $\mathrm{Al}_{2} \mathrm{O}_{3}$ & 18.41 & 18.42 & 19.15 & 19.52 & 19.74 & 20.02 & 20.31 & 20.15 & 20.04 & 20.26 \\
\hline $\mathrm{MgO}$ & 14.66 & 14.44 & 14.41 & 14.48 & 14.65 & 14.60 & 14.20 & 14.46 & 14.41 & 14.26 \\
\hline $\mathrm{FeO}$ & 5.75 & 5.68 & 5.45 & 5.16 & 5.17 & 5.18 & 5.07 & 4.99 & 4.96 & 5.01 \\
\hline $\mathrm{CaO}$ & 10.06 & 10.31 & 10.55 & 10.89 & 10.95 & 11.12 & 11.47 & 11.48 & 11.57 & 11.66 \\
\hline $\mathrm{Na}_{2} \mathrm{O}$ & 2.93 & 2.90 & 3.05 & 3.08 & 3.13 & 3.20 & 3.23 & 3.19 & 3.15 & 3.19 \\
\hline $\mathrm{K}_{2} \mathrm{O}$ & 0.82 & 0.83 & 0.84 & 0.85 & 0.85 & 0.86 & 0.88 & 0.89 & 0.89 & 0.87 \\
\hline \multicolumn{11}{|l|}{ Std. Dev. } \\
\hline $\mathrm{SiO}_{2}$ & 7.21 & 7.22 & 7.26 & 7.24 & 7.26 & 7.13 & 7.22 & 7.11 & 7.13 & 7.27 \\
\hline $\mathrm{Al}_{2} \mathrm{O}_{3}$ & 6.49 & 6.50 & 6.38 & 6.25 & 6.26 & 6.22 & 6.40 & 6.27 & 6.23 & 6.30 \\
\hline $\mathrm{MgO}$ & 7.90 & 7.64 & 7.92 & 7.96 & 7.92 & 7.81 & 7.71 & 7.62 & 7.67 & 7.63 \\
\hline $\mathrm{FeO}$ & 4.08 & 4.13 & 3.94 & 3.76 & 3.78 & 3.83 & 3.77 & 3.69 & 3.67 & 3.64 \\
\hline $\mathrm{CaO}$ & 6.30 & 6.11 & 6.22 & 6.19 & 6.01 & 6.00 & 5.95 & 5.93 & 5.93 & 5.85 \\
\hline $\mathrm{Na}_{2} \mathrm{O}$ & 1.78 & 1.76 & 1.72 & 1.69 & 1.71 & 1.68 & 1.71 & 1.69 & 1.67 & 1.68 \\
\hline $\mathrm{K}_{2} \mathrm{O}$ & 0.63 & 0.64 & 0.63 & 0.62 & 0.62 & 0.62 & 0.62 & 0.62 & 0.62 & 0.62 \\
\hline$N$ & 239 & 235 & 227 & 232 & 229 & 234 & 235 & 230 & 230 & 233 \\
\hline
\end{tabular}


Table A.3: continued

\begin{tabular}{|c|c|c|c|c|c|c|c|c|c|c|}
\hline Depth $(\mathrm{km})$ & 5 & 10 & 15 & 20 & 25 & 30 & 35 & 40 & 45 & 50 \\
\hline $\mathrm{T}(\mathrm{C})$ & 135.97 & 237.53 & 327.32 & 409.97 & 488.29 & 563.98 & 638.08 & 711.21 & 783.76 & 855.95 \\
\hline $\mathrm{P}$ (kbar) & 1.62 & 3.23 & 4.85 & 6.47 & 8.09 & 9.70 & 11.32 & 12.94 & 14.55 & 16.17 \\
\hline \multicolumn{11}{|c|}{$V_{p}=7.6 \mathrm{~km} / \mathrm{s}$} \\
\hline Mean & & & & & & & & & & \\
\hline $\mathrm{SiO}_{2}$ & 44.55 & 44.10 & 43.16 & 42.92 & 42.33 & 41.92 & 41.71 & 41.68 & 41.38 & 41.37 \\
\hline $\mathrm{Al}_{2} \mathrm{O}_{3}$ & 20.48 & 20.67 & 21.49 & 21.54 & 21.62 & 21.92 & 21.88 & 21.77 & 21.79 & 21.84 \\
\hline $\mathrm{MgO}$ & 14.52 & 14.69 & 14.37 & 14.40 & 14.94 & 14.78 & 15.12 & 15.32 & 15.71 & 15.72 \\
\hline $\mathrm{FeO}$ & 5.32 & 5.07 & 5.19 & 5.15 & 5.44 & 5.52 & 5.43 & 5.51 & 5.54 & 5.48 \\
\hline $\mathrm{CaO}$ & 10.82 & 11.17 & 11.30 & 11.51 & 11.19 & 11.35 & 11.34 & 11.22 & 11.06 & 11.07 \\
\hline $\mathrm{Na}_{2} \mathrm{O}$ & 3.40 & 3.39 & 3.59 & 3.57 & 3.63 & 3.68 & 3.68 & 3.67 & 3.69 & 3.70 \\
\hline $\mathrm{K}_{2} \mathrm{O}$ & 0.93 & 0.92 & 0.92 & 0.92 & 0.86 & 0.84 & 0.85 & 0.84 & 0.84 & 0.84 \\
\hline \multicolumn{11}{|l|}{ Std. Dev. } \\
\hline $\mathrm{SiO}_{2}$ & 7.31 & 7.13 & 7.60 & 7.61 & 7.81 & 7.94 & 7.75 & 7.78 & 7.67 & 7.64 \\
\hline $\mathrm{Al}_{2} \mathrm{O}_{3}$ & 6.41 & 6.42 & 6.79 & 6.77 & 6.76 & 6.69 & 6.69 & 6.68 & 6.68 & 6.46 \\
\hline $\mathrm{MgO}$ & 7.72 & 7.53 & 7.30 & 7.03 & 7.18 & 7.11 & 7.29 & 7.24 & 7.59 & 7.61 \\
\hline $\mathrm{FeO}$ & 3.93 & 3.57 & 3.51 & 3.49 & 3.48 & 3.47 & 3.41 & 3.46 & 3.47 & 3.44 \\
\hline $\mathrm{CaO}$ & 5.89 & 5.71 & 5.54 & 5.38 & 5.29 & 5.21 & 5.18 & 5.11 & 5.25 & 5.26 \\
\hline $\mathrm{Na}_{2} \mathrm{O}$ & 1.68 & 1.69 & 1.75 & 1.78 & 1.79 & 1.76 & 1.75 & 1.75 & 1.73 & 1.72 \\
\hline $\mathrm{K}_{2} \mathrm{O}$ & 0.74 & 0.73 & 0.73 & 0.72 & 0.72 & 0.73 & 0.73 & 0.73 & 0.72 & 0.72 \\
\hline$N$ & 205 & 208 & 228 & 240 & 251 & 263 & 259 & 260 & 258 & 264 \\
\hline
\end{tabular}




\section{Bibliography}

Ackermann, R., and R. Schlische, Uh-Oh! N<1: Dynamic length-displacement scaling, Eos Trans. AGU, 80, S328, 1999.

Alexander, R., and K. Macdonald, Sea Beam, Sea MARC II, and ALVIN-based investigations of faulting on the East Pacific Rise $9^{\circ} 20^{\prime}-9^{\circ} 50^{\prime} \mathrm{N}$, Mar. Geophys. Res., 18, 557-587, 1996.

Allerton, S., B. Murton, R. Searle, and M. Jones, Extensional faulting and segmentation of the Mid-Atlantic Ridge north of the Kane fracture zone $\left(24^{\circ} 00^{\prime} \mathrm{N}\right.$ to $\left.24^{\circ} 40^{\prime} \mathrm{N}\right)$, Mar. Geophys. Res., 17, 37-61, 1995.

Alsop, L., and M. Talwani, The East Coast Magnetic Anomaly, Science, 226, 1189-1191, 1984.

Anderson, E., The Dynamics of Faulting and Dyke Formation with Applications to Britain, Oliver and Boyd, Edinburgh, 1951.

Austin, J., J.A., P. Stoffa, J. Phillips, J. Oh, D. Sawyer, G. Purdy, E. Reiter, and J. Makris, Crustal structure of the southeast Georgia embayment-Carolina Trough: Preliminary results of a composite seismic image of a continental suture(?) and a volcanic passive margin, Geology, 18, 1023-1027, 1990.

Austrheim, H., The influence of fluid and deformation on metamorphism of the depp crust and consequences for geodynamics of collision zones, in When Continents Collide: Geodynamics and Geochemsitry of Ultrahigh-Pressure Rocks, edited by B. Hacker and J. Liou, pp. 297-323, Kluwer Academic Publishers, Dordrecht, 1998.

Baker, H. M., M.B., M. Ghiorso, and E. Stolper, Compositions of near-solidus peridotite melts from experiments and thermodynamic calculations, Nature, 375, 308-311, 1995.

Baker, M., and E. Stolper, Determining the composition of high-pressure mantle melts using diamond aggregates, Geochim. Cosmochim. Acta, 58, 2811-2827, 1994.

Barclay, A., D. Toomey, and S. Solomon, Microearthquake characteristics and crustal Vp/Vs structure at the Mid-Atlantic Ridge, 35 N, J. Geophys. Res., 106, 2017-2034, 2001.

Barnett, J., J. Mortimer, J. Rippon, J. Walsh, and J. Watterson, Displacement geometry in the volume containing a single normal fault, Am. Assoc. Petro. Geol. Bull., 71, 925-937, 1987.

Bass, J., Elasticity of minerals, glasses, and melts, in A Handbook of Physical Constants: Mineral Physics and Crystallography, edited by T. Ahrens, pp. 45-63, Am. Geophys. Union, Washington, D.C., 1995. 
Bathe, K.-J., Finite Element Procedures, Prentice Hall, New Jersey, 1996.

Bercovici, D., H. Dick, and T. Wagner, Nonlinear viscoelasticity and the formation of transverse ridges, J. Geophys. Res., 97, 14,195-14,206, 1992.

Bergman, E., and S. Solomon, Transform fault earthquakes in the North Atlantic: Source mechanisms and depth of faulting, J. Geophys. Res., 93, 9027-9057, 1988.

Bernstein, S., High-pressure fractionation in rift-related basaltic magmatism; Faeroe plateau basalts, Geology, 22, 815-818, 1994.

Berryman, J., Mixture theories for rock properties, in A Handbook of Physical Constants: Rock Physics and Phase Relations, edited by T. Ahrens, pp. 205-228, Am. Geophys. Union, Washington, D.C., 1995.

Bicknell, J., J.-C. Sempéré, K. Macdonald, and P. Fox, Tectonics of a fast spreading center: A deep-tow and Sea Beam survey on the East Pacific Rise at 19³0'S, Mar. Geophys. Res., 9, 25-45, 1987.

Birch, F., Intepretation of the seismic structure of the crust in light of experimental studies of wave velocities in rocks, in Contributions in Geophysics in Honor of Beno Gutenberg, edited by H. Benioff, pp. 158-170, Pergamon, New York, 1958.

Birch, F., The velocity of compressional waves in rocks to 10 kilobars, part 1, J. Geophys. Res., $65,1083-1102,1960$.

Birch, F., The velocity of compressional waves in rocks to 10 kilobars, part 2, J. Geophys. Res., 66, 2199-2224, 1961.

Blackman, D., and D. Forsyth, Isostatic compensation of tectonic features of the Mid-Atlantic Ridge: 25-27³0's, J. Geophys. Res., 96, 11,741,11,758, 1991.

Blakely, R., Potential Theory in Gravity and Magnetic Applications, Cambridge Univ. Press, New York, 1995.

Boettcher, M., and T. Jordan, Seismic behavior of oceanic transform faults, Eos. Trans. AGU, 82, S32E-07, 2001.

Bohnenstiehl, D., and M. Kleinrock, Faulting and fault scaling on the median valley floor of the trans-Atlantic geotraverse (TAG) segment, $\sim 26^{\circ} \mathrm{N}$ on the Mid-Atlantic Ridge, J. Geophys. Res., 104, 29,351-29,364, 1999.

Bott, M., Crustal doming and the mechanism of continental rifting, Tectonophys., 73, 1-8, 1981 .

Boutilier, R., and C. Keen, Geodynamic models of fault-controlled extension, Tectonics, 13, 439_ 454, 1994.

Boutilier, R., and C. Keen, Small-scale convection and divergent plate boundaries, J. Geophys. Res., 104, 7389-7403, 1999.

Brace, W., and D. Kohlstedt, Limits on lithospheric stress imposed by laboratory experiments, $J$. Geophys. Res., 85, 6248-6252, 1980. 
Braile, L., B. Wang, C. Daudt, G. Keller, and J. Patel, Modeling the 2-D seismic velocity structure across the Kenya rift, Tectonophys., 236, 251-269, 1994.

Brandle, J., and G. Nagy, The state of the 5th Version of the IGBA: Igneous Petrological Data Base, Comp. and Geosci., 21, 425-432, 1995.

Bratt, S., and S. Solomon, Compressional and shear wave structure of the East Pacific Rise at $11^{\circ} 20^{\prime} \mathrm{N}$ : Constraints from three-component ocean bottom seismometer data, J. Geophys. Res., $89,6095-6110,1984$.

Braun, J., and C. Beaumont, A physical explanation of the relation between flank uplifts and the breakup unconformity at rifted continental margins, Geology, 17, 76-764, 1989.

Briais, A., H. Sloan, L. Parson, and B. Murton, Accretionary processes in the axial valley of the Mid-Atlantic Ridge $27^{\circ} \mathrm{N}-30^{\circ} \mathrm{N}$ from TOBI side-scan sonar images, Mar. Geophys. Res., 21, 87$119,2000$.

Browning, P., The petrology, geochemistry, and structure of the plutonic rocks of the Oman ophiolite, $\mathrm{PhD}$ thesis, Open Univ., Milton Keynes, England, 1982.

Brune, J., Seismic moment, seismicity, and rate of slip along major fault zones, J. Geophys. Res., 73, 777-784, 1968.

Buck, W., Modes of continental lithospheric extension, J. Geophys. Res., 96, 20,161-20,178, 1991.

Buck, W., Effect of lithospheric thickness on the formation of high- and low-angle normal faults, Geology, 21, 933-936, 1993.

Buck, W., and A. Poliakov, Abyssal hills formed by stretching oceanic lithosphere, Nature, 392, 272-275, 1998.

Burchfiel, B., and J. Stewart, Pull-apart origin of the central segment of Death Valley, California, Geol. Soc. Am. Bull., 77, 439-441, 1966.

Burgess, C., B. Rosendahl, S. Sander, C. Burgess, J. Lambiase, S. Derksen, and N. Meader, The structural and stratigraphic evolution of Lake Tanganyika: A case study of continental rifting, in Triassic-Jurassic Rifting: Continental Breakup and the Origin of the Atlantic Ocean and Passive Margin, Part B, edited by W. Manspeizer, pp. 859-881, Elsevier, 1988.

Burke, M., and D. Fountain, Seismic properties of rocks from an exposure of extended continental crust-new laboratory measurements from the Ivrea Zone, Tectonophys., 182, 119-146, 1990.

Burns, L., The Border Ranges ultramafic and mafic complex: plutonic core of an intraoceanic island arc, $\mathrm{PhD}$ dissertation, Stanford Univ., 1983.

Byerlee, J., Friction of rocks, Pure Appl. Geophys., 116, 615-626, 1978.

Campbell, D., Investigation of the stress-concentration mechanism for intraplate earthquakes, Geophys. Res. Lett., 5, 477-479, 1978. 
Canales, J., J. Collins, J. Escartín, and R. Detrick, Seismic structure across the rift valley of the Mid-Atlantic Ridge at $23^{\circ} 20^{\prime} \mathrm{N}$ (MARK Area): Implications for crustal accretion processes at slow-spreading ridges, J. Geophys. Res., 105, 28,411-28,425, 2000a.

Canales, J., R. Detrick, J. Lin, J. Collins, and D. Toomey, Crustal and upper mantle seismic structure beneath the rift mountains and across a nontransform offset at the Mid-Atlantic Ridge $\left(35^{\circ} \mathrm{N}\right)$, J. Geophys. Res., 105, 2699-2719, 2000b.

Canales, J., R. Detrick, D. Toomey, and W. Wilcock, Segment-scale variations in crustal structure of 150 - to $300-\mathrm{k}$.y.-old fast spreading oceanic crust (East Pacific Rise, $8^{\circ} 15^{\prime} \mathrm{N}-10^{\circ} 15^{\prime} \mathrm{N}$ ) from wide-angle seismic refraction profiles, submitted to Geophys. J. Int., 2001.

Cannat, M., et al., Thin crust, ultramafic exposures, and rugged faulting patterns at the MidAtlantic Ridge $\left(22^{\circ}-24^{\circ} \mathrm{N}\right)$, Geology, 23, 49-52, 1995.

Carbotte, S., and K. Macdonald, Causes of variation in fault-facing direction on the ocean floor, Geology, 18, 749-752, 1990.

Carbotte, S., and K. Macdonald, Comparison of seafloor tectonic fabric at intermediate, fast, and super fast spreading ridges: Influence of spreading rate, plate motions, and ridge segmentation on fault patterns, J. Geophys. Res., 99, 13,609-13,631, 1994.

Caristan, Y., The transition from high temperature creep to fracture in Maryland diabase, J. Geophys. Res., 87, 6781-6790, 1982.

Carlson, R., The abundance of ultramafic rocks in Atlantic Ocean crust, Geophys. J. Int., 144, 37-48, 2001.

Carlson, R., and J. Miller, A new assessment of the abundance of serpentinites in the oceanic crust, Geophys. Res. Lett., 24, 457-40, 1997.

Casey, J., Comparison of major- and trace-element geochemistry of abyssal peridotites and mafic plutonic rocks with basalts from the MARK Region of the Mid-Atlantic Ridge, in Proc. Ocean Drill. Program, Sci. Res., edited by J. Karson, M. Cannat, D. Miller, and D. Elthon, vol. 153, pp. 181-241, College Station, TX, 1997.

Cermak, V., L. Bodri, R. Schulz, and B. Tanner, Crustal temperatures along the central segment of the European Geotraverse, Tectonophys., 195, 241-251, 1991.

Chapin, C., and S. Cather, Tectonic setting of the axial basins of the northern and central Rio Grande rift, in Basins of the Rio Grande rift: Structure, stratigraphy, and tectonic setting, edited by G. Keller and S. Cather, vol. 291, pp. 5-25, GSA Spec. Paper, Boulder, 1994.

Chayes, F., IGBADAT: A world data base for igneous petrology, Episodes, 8, 245-251, 1985.

Chen, W.-P., and P Molnar, Focal depths of intracontinental and intraplate earthquakes and their implications for the thermal and mechanical properties of the lithosphere, J. Geophys. Res., 88, 4183-4214, 1983.

Chen, Y., A mechanical model for the inside corner uplift at a ridge-transform intersection, $J$. Geophys. Res., 94, 9275-9282, 1989. 
Chen, Y., and J. Lin, Mechanisms for the formation of ridge-axis topography at slow-spreading ridges: a lithospheric-plate flexure model, Geophys. J. Int., 136, 8-18, 1999.

Chen, Y., and W. Morgan, A nonlinear rheology model for mid-ocean ridge axis topography, $J$. Geophys. Res., 95, 17,583-17,604, 1990.

Cheng, C., and D. Johnston, Dynamic and static moduli, Geophys. Res. Lett., 8, 39-42, 1981.

Chéry, J., Core complex mechanics: From the Gulf of Corinth to the Snake Range, Geology, 29, 439-442, 2001.

Chopra, P., and M. Paterson, The role of water in the deformation of dunite, J. Geophys. Res., 89, 7861-7876, 1984.

Christensen, N., Compressional wave velocities in metamorphic rocks at pressures to 10 kilobars, J. Geophys. Res., 70, 6147-6164, 1965.

Christensen, N., Ophiolites, seismic velocities and oceanic crustal structure, Tectonophys., 47, 131-157, 1978.

Christensen, N., Compressional wave velocities in rocks at high temperature and pressures, critical thermal gradients, and crustal low-velocity zones, J. Geophys. Res., 84, 6849-6857, 1979.

Christensen, N., Reflectivity and seismic properties of the deep continental crust, J. Geophys. Res., 94, 17,793-17,804, 1989.

Christensen, N., and W. Mooney, Seismic velocity structure and composition of the continenetal crust: A global view, J. Geophys. Res., 100, 9761-9788, 1995.

Christensen, N., and M. Salisbury, Structure and constitution of the lower oceanic crust, Rev. Geophys., 13, 57-86, 1975.

Christensen, N., and J. Smewing, Geology and seismic structure of the northern section of the Oman ophiolite, J. Geophys. Res., 86, 2545-2555, 1981.

Christensen, N., and D. Szymanski, Origin of reflections from the Brevard fault zone, J. Geophys. Res., 93, 1087-1102, 1988.

Cochran, J., and F. Martinez, Evidence from the northern Red Sea on the transition from continental to oceanic rifting, Tectonophys., 153, 25-53, 1988.

Collier, J., and S. Singh, Poisson's ratio structure of young oceanic crust, J. Geophys. Res., 103, 20,981-20,996, 1998.

Connolly, J., Multivariable phase diagrams: an algorithm based on generalized thermodynamics, Am. J. Sci., 290, 666-718, 1990.

Courtillot, V., P. Tapponnier, and J. Varet, Surface features associated with transform faults: A comparison between observed examples and an experimental model, Tectonophys., 24, 317-329, 1974. 
Cowie, P., Normal fault growth in three-dimensions in continental and oceanic crust, in Faulting and Magmatism at Mid-Ocean Ridges Geophys. Mono. Ser, edited by W. Buck, P. Delaney, J. Karson, and Y. Lagabrielle, vol. 106, pp. 325-348, Am. Geophys. Union, Washington, D.C., 1998.

Cowie, P., C. Scholz, M. Edwards, and A. Malinverno, Fault strain and seismic coupling on midocean ridges, J. Geophys. Res., 98, 17,911-17,920, 1993.

Crider, J., and D. Pollard, Fault linkage: Three-dimensional mechanical interaction between echelon normal faults, J. Geophys. Res., 103, 24,373-24,391, 1998.

DeBari, S., and N. Sleep, High-Mg, low-Al bulk composition of the Talkeetna island arc, Alaska: implications for primary magmas and the nature of arc crust, Geol. Soc. Am. Bull., 103, 37-47, 1991.

Decker, E., and S. Smithson, Heat flow and gravity interpretation across the Rio Grande rift in southern New Mexico and west Texas, J. Geophys. Res., 80, 2542-2552, 1975.

Degens, E., R. Von Herzen, and H. Wong, Lake Tanganyika: Water chemistry, sediments, geologic structure, Naturwissenschaften, 58, 229-240, 1971.

Degens, E., R. Von Herzen, H. Wong, W. Denser, and H. Jannasch, Lake Kivu: Structure, chemistry and biology of an East African lake, Geol. Runsch., 62, 245-277, 1973.

Detrick, R., H. Needham, and V. Renard, Gravity anomalies and crustal thickness variations along the Mid-Atlantic Ridge between $33^{\circ} \mathrm{N}$ and $40^{\circ} \mathrm{N}$, J. Geophys. Res., 100, 3767-3787, 1995.

Diament, M., and M. Kogan, Long wavelength gravity anomalies and the deep thermal structure of the Baikal rift, Geophys. Res. Lett., 17, 1977-1980, 1990.

Dick, H., et al., A long in situe section of the lower ocean crust: results of ODP Leg 176 drilling at the Southwest Indian Ridge, Earth Planet. Sci. Lett., 179, 31-51, 2000.

Dieterich, J., Time-dependent friction in rocks, J. Geophys. Res., 77, 3690-3697, 1972.

Dieterich, J., Modeling of rock friction 1. Experimental results and constitutive equations, J. Geophys. Res., 84, 2161-2168, 1979.

Dietz, R., Alpine serpentinites as oceanic rind fragments, Geol. Soc. Am. Bull., 74, 947-952, 1963.

Dillon, W., and P. Popenoe, The blake plateau basin and carolina trough, in The Geology of North America, edited by R. Sheridan and J. Grow, vol. The Atlantic Continental Margin, v. I-2, pp. 291-328, Geological Society of America, 1988.

Dunbar, J., and D. Sawyer, How preexisting weaknesses control the style of continental breakup, J. Geophys. Res., 94, 7278-7292, 1989.

Dupuy, C., A. Leyreloup, and J. Vernieres, The lower continental crust of the Massif Central (Bournac, France: with special references to REE, U and Th composition, evolution, and heatflow production, Phys. Chem. Earth, 11, 401-415, 1979. 
Ebinger, C., Tectonic development of the western branch of the East African rift system, Geol. Soc. Am. Bull., 101, 885-903, 1989.

Ebinger, C., and A. Ibrahim, Multiple episodes of rifting in Central and East Africa: A reevaluation of gravity data, Geol. Rundsch, 83, 689-702, 1994.

Ebinger, C., B. Rosendahl, and D. Reynolds, Tectonic model of the Malawi rift, Africa, Tectonophys., 141, 215-235, 1987.

Ebinger, C., T. Bechtel, D. Forsyth, and C. Bowin, Effective elastic plate thickness beneath the East African and Afar plateaus and dynamic compensation of the uplifts, J. Geophys. Res., 94, 2883-2901, 1989.

Edwards, M., D. Fornari, A. Malinverno, and W. Ryan, The regional tectonic fabric of the East Pacific Rise from $12^{\circ} 50^{\prime} \mathrm{N}$ to $15^{\circ} 10^{\prime} \mathrm{N}$, J. Geophys. Res., 96, 7995-8017, 1991.

Eissa, E., and A. Kazi, Relation between static and dynamic Young's modulus, Int. J. Rock Mech. Min. Sci. Geomech. Abstr., 25, 479-482, 1988.

Engeln, J., D. Wiens, and S. Stein, Mechanisms and depths of Atlantic transform earthquakes, $J$. Geophys. Res., 91, 548-577, 1986.

England, P., Constraints on extension of continental lithosphere, J. Geophys. Res., 88, 1145-1152, 1983.

Escartín, J., and J. Lin, Ridge offsets, normal faulting, and gravity anomalies of slow spreading ridges, J. Geophys. Res., 100, 6163-6177, 1995.

Escartín, J., and J. Lin, Tectonic modification of axial crustal structure: Evidence from spectral analysis of residual gravity and bathymetry of the Mid-Atlantic Ridge flanks, Earth Planet. Sci. Lett., 154, 279-293, 1998.

Escartín, J., G. Hirth, and B. Evans, Effects of serpentinization on the lithospheric strength and the style of normal faulting at slow-spreading ridges, Earth Planet. Sci. Lett., 151, 181-189, 1997.

Escartín, J., P. Cowie, R. Searle, S. Allerton, N. Mitchell, C. Macleod, and A. Slootweg, Quantifying tectonic strain and magmatic accretion at a slow spreading ridge segment, Mid-Atlantic Ridge, $29^{\circ}$ N, J. Geophys. Res., 104, 10,421-10,437, 1999.

Fletcher, R., and B. Hallet, Unstable extension of the lithosphere: A mechanical model for Basin and Range structure, J. Geophys. Res., 88, 7457-7466, 1983.

Fliedner, M., and S. Klemperer, Structure of an island-arc: Wide-angle seismic studies in the eastern Aleutian Islands, Alaska, J. Geophys. Res., 104, 10,667-10,694, 1999.

Fliedner, M., and S. Klemperer, Crustal structure transition from oceanic arc to continental arc, eastern Aleutian Islands and Alaskan Peninsula, Earth Planet. Sci. Lett., 179, 567-579, 2000.

Forslund, T., and A. Gudmundsson, Crustal spreading due to dikes and faults in southwest Iceland, J. Struct. Geol., 13, 443-457, 1991. 
Forsyth, D., Finite extension and low-angle normal faulting, Geology, 20, 27-30, 1992.

Fountain, D., and N. Christensen, Composition of the continental crust and upper mantle; A review, Mem. Geol. Soc. Am., 172, 711-742, 1989.

Fountain, D., M. Salisbury, and J. Percival, Seismic structure of the continental crust based on rock velocity measurements from the Kapuskasing uplift, J. Geophys. Res., 95, 1167-1186, 1990.

Fox, P., and D. Gallo, A tectonic model for ridge-transform-ridge plate boundaries: Implications for the structure of oceanic litosphere, Tectonophys., 104, 205-242, 1984.

Frederiksen, S., and J. Braun, Numerical modelling of strain localisation during extension of the continental lithosphere, Earth Planet. Sci. Lett., 188, 241-251, 2001.

Fujita, K., and N. Sleep, Membrane stressesnear mid-ocean ridge-transform intersections, Tectonophys., 50, 207-221, 1978.

Gaulier, J., X. Le Pichon, N. Lyberis, F. Avedik, L. Geli, I. Moretti, A. Deschamps, and S. Hafez, Seismic study of the crust of the northern Red Sea and Gulf of Suez, Tectonophys., 153, 55-88, 1988.

Gerbault, M., E. Burov, A. Poliakov, and M. Daignières, Do faults trigger folding in the lithosphere?, Geophys. Res. Lett., 26, 271-274, 1999.

Goetze, C., The mechanisms of creep in olivine, Phil. Trans. R. Soc. Lond. A., 288, 99-119, 1978.

Goetze, C., and B. Evans, Stress and temperature in the bending lithosphere as constrained by experimental rock mechanics, Geophys. J.R. astr. Soc., 59, 463-478, 1979.

Goff, J., Y. Ma, A. Shah, J. Cochran, and J.-C. Sempéré, Stochastic analysis of seafloor morphology on the flank of the Southeast Indian Ridge: The influence of ridge morphology on the formation of abyssal hills, J. Geophys. Res., 102, 15,521-15,534, 1997.

Gomberg, J., and M. Ellis, Topography and tectonics of the central New Madrid seismic zone: Results of numerical experiments using a three-dimensional boundary element program, J. Geophys. Res., 99, 20,299-20,310, 1994.

Gràcia, E., D. Bideau, R. Hekinian, and Y. Lagabrielle, Detailed geological mapping of two contrasting second-order segments of the Mid-Atlantic Ridge between Oceanographer and Hayes frac-

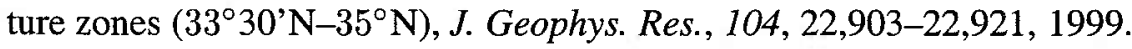

Grindlay, N., and P. Fox, Lithospheric stresses associated with nontransform offsets of the MidAtlantic Ridge: Implications from a finite element analysis, Tectonics, 12, 982-1003, 1993.

Grindlay, N., P. Fox, and K. Macdonald, Second-order ridge axis discontinuities in the South Atlantic: Morphology, structure, and evolution, Mar. Geophys. Res., 13, 21-49, 1991.

Grindlay, N., P. Fox, and P. Vogt, Morphology and tectonics of the Mid-Atlantic Ridge $\left(25^{\circ}-\right.$ $27^{\circ} 30^{\prime}$ S) from Sea Beam and magnetic data, J. Geophys. Res., 97, 6983-7010, 1992. 
Grindlay, N., J Madsen, C. Rommevaux-Jestin, and J. Sclater, A different pattern of ridge segmentation and mantle Bouguer gravity anomalies along the ultra-slow spreading Southwest Indian Ridge, Earth Planet. Sci. Lett., 161, 243-253, 1998.

Gudmundsson, A., Effect of tensile stress concentration around magma chambers of intrusion and extrusion frequencies, J. Volc. Geotherm. Res., 35, 179-194, 1988.

Gudmundsson, A., Stress fields associated with oceanic transform faults, Earth Planet. Sci. Lett., 136, 603-614, 1995.

Gutscher, M.-A., Crustal structure and dynamics in the Rhine Graben and the Alpine foreland, Geophys. J. Int., 122, 617-636, 1995.

Hacker, B., E. Gnos, L. Ratschbacher, M. Grove, M. McWilliams, S. Sobolev, J. Wan, and W. Zhenhan, Hot and dry deep crustal xenoliths from Tibet, Science, 287, 2463-2466, 2000.

Hansen, F., and N. Carter, Semibrittle creep of dry and wet Westerly granite at $1000 \mathrm{MPa}$, pp. 429-447, 24th U.S. Symposium on Rock Mechanics, Texas A\&M Univ., College Station, Texas, 1983.

Hashin, Z., and S. Shtrikman, A variational approach to the elastic behavior of multiphase materials, J. Mech. Phys. Solids, 11, 127-140, 1963.

Hayward, N., and C. Ebinger, Variations in the along-axis segmentation of the Afar rift system, Tectonics, 15, 244-257, 1996.

Hekinian, R., D. Bideau, J. Francheteau, J. Cheminee, R. Armijo, P. Lonsdale, and N. Blum, Petrology of the East Pacific Rise crust and upper mantle exposed in Hess Deep (Eastern Equatorial Pacific), J. Geophys. Res., 98, 8069-8094, 1993.

Hess, H., History of ocean basins, in Petrologic Studies, edited by A. Engel, H. James, and B. Leonard, pp. 599-620, GSA, Boulder, CO, 1962.

Hess, H., Mid-ocean ridges and tectonics of the seafloor, in Submarine Geology and Geophysics, edited by W. Whittard and R. Bradshaw, pp. 317-333, Colston Research Symposium, Butterworths, London, 1965.

Hinz, K., A hypothesis on terrestrial catastrophes: Wedges of very thick oceanward dipping layers beneath passive margins, Geol. Jahrb., 22, 5-28, 1981.

Hirose, K., and I. Kushiro, Partial melting of dry peridotites at high pressures: Determination of compositions of melts segregated from peridotite using aggregates of diamond, Earth Planet. Sci. Lett., 114, 477-489, 1993.

Hirschmann, M., M. Baker, and E. Stopler, The effect of alkalis on the silica content of mantlederived melts, Geochim. Cosmochim. Acta, 62, 883-902, 1998.

Hirth, G., J. Escartin, and J. Lin, The rheology of the lower oceanic crust: Implications for lithospheric deformation at mid-ocean ridges, in Faulting and Magmatism at Mid-Ocean Ridges, Geophys. Mono. Ser, edited by W. Buck, P. Delaney, J. Karson, and Y. Lagabrielle, vol. 106, pp. 291-303, AGU, Washington, D.C., 1998. 
Holbrook, W., and P. Kelemen, Large igneous province on the US Atlantic margin and implications for magmatism during continental breakup, Nature, 364, 433-436, 1993.

Holbrook, W., W. Mooney, and N. Christensen, The seismic velocity structure of the deep continental crust, in Continental Lower Crust, edited by D. Fountain, R. Arculus, and R. Kay, pp. 1-43, Elsevier Sci., New York, 1992.

Holbrook, W., G. Purdy, R. Sheridan, I. Glover, L., M. Talwani, J. Ewing, and D. Hutchinson, Seismic structure of the U.S. mid-Atlantic continental margin, J. Geophys. Res., 99, 17,871-17,891, 1994a.

Holbrook, W., E. Reiter, G. Purdy, D. Sawyer, P. Stoffa, J. Austin, J.A., J. Oh, and J. Makris, Deep structure of the U.S. Atlantic continental margin, offshore South Carolina, from coincident ocean bottom and multichannel seismic data, J. Geophys. Res., 99, 9155-9178, 1994b.

Holbrook, W., D. Lizarralde, S. McGeary, N. Bangs, and J. Diebold, Structure and composition of the Aleutian island arc and implications for continental crustal growth, Geology, 27, 31-34, 1999.

Holbrook, W., et al., Mantle thermal structure and active upwelling during continental breakup in the North Atlantic, Earth Planet. Sci. Lett., 190, 251-266, 2001.

Holland, T., and R. Powell, An internally consistent thermodynamic data set for phases of petrological interest, J. Metamorp. Geol., 16, 309-343, 1998.

Hooft, E., R. Detrick, D. Toomey, J. Collins, and J. Lin, Crustal thickness and structure along three contrasting spreading segments of the Mid-Atlantic Ridge, $33.5^{\circ}-35^{\circ} \mathrm{N}, J$. Geophys. Res., $105,8205-8226,2000$.

Hopper, J., J. Mutter, R. Larson, C. Mutter, and N. A. S. Group, Magmatism and rift margin evolution: Evidence from northwest Australia, Geology, 20, 853-857, 1992.

Horen, H., M. Zamora, and G. Dubuisson, Seismic wave velocities and anisotropy in serpentinized peridotites from Xigaze ophiolite: Abundance of serpentinite in slow spreading ridge, Geophys. Res. Lett., 23, 9-12, 1996.

Horsefield, S., R. Whitmarsh, R. White, and J.-C. Sibuet, Crustal structure of the Goban Spur rifted continental margin, NE Atlantic, Geophys. J. Int., 119, 1-19, 1993.

Huang, P., and S. Solomon, Centroid depths and mechanisms of mid-ocean ridge earthquakes in the Indian Ocean, Gulf of Aden, and the Red Sea, J. Geophys. Res., 92, 1361-1382, 1987.

Huang, P., and S. Solomon, Centroid depths of mid-ocean ridge earthquakes: Dependence of spreading rate, J. Geophys. Res., 93, 13,445-13,477, 1988.

Huang, P., S. Solomon, E. Bergman, and J. Nabelek, Focal depths and mechanisms of Mid-Atlantic Ridge earthquakes from body waveform inversion, J. Geophys. Res., 91, 579-598, 1986.

Huenges, E., J. Lauterjung, C. Bücker, E. Lippman, and H. Kern, Seismic velocity, density, thermal conductivity and heat production of cores from the KTB pilot hole, Geophys. Res. Lett., 24, 345348, 1997. 
Hutchinson, D., J. Grow, K. Klitgord, and B. Swift, Deep structure and evolution of the Carolina trough, in Studies in Continental Margin Geology, edited by J. Watkins and C. Drake, AAPG Memoir, 34 ed., pp. 129-152, 1983.

Hutchinson, D., A. Golmshtok, L. Zonenshain, T. Moore, C. Scholz, and K. Klitgord, Depositional and tectonic framework of the rift basins of Lake Baikal from multichannel seismic data, Geology, 20, 589-592, 1992.

Hutchinson, D., C. Poag, A. Johnson, P. Popenoe, and C. Wright, Geophysical database of the East Coast of the United States: Southern Atlantic Margin-Stratigraphy and velocity in map grids, Open File 96-55, U.S. Geol. Surv. Open File Report, 1996.

Hyndman, D., Petrology of igneous and metamorphic rocks, McGraw-Hill, Inc., New York, 1985.

Iturrino, G., N. Christensen, S. Kirby, and M. Salisbury, Seismic velocities and elastic properties of oceanic gabbroic rocks from hole 735b, in Proc. Ocean Drill. Program, Sci. Res., edited by R. Von Herzen, P. Robinson, A. Adamson, K. Becker, S. Bloomer, M. Cannat, H. Dick, R. Emmermann, G. Gard, D. Goldberg, R. Hebert, J. Hertogen, H. Hoskins, G. Iturrino, J. Kassenaar, P. Kempton, E. Kikawa, S. Kirby, P. Meyer, J. Natland, K. Ozawa, J. Pariso, J. Scott, D. Stakes, S. Swift, and S. Stewart, vol. 118, pp. 227-244, College Station, TX, 1991.

Iturrino, G., D. Miller, and N. Christensen, Velocity behavoir of lower crustal and upper mantle rocks from a fast-spreading ridge at Hess Deep, in Proc. Ocean Drill. Program, Sci. Res., edited by C. Mével, K. Gillis, J. Allan, and P. Meyer, vol. 147, pp. 417-440, College Station, TX, 1996.

Jackson, I., The petrophysical basis for the interpretation of seismological models of the continental lithosphere, Spec. Pub. Geol. Soc. Aust., 17, 81-114, 1991.

Jackson, I., R. Rudnick, S. O'Reilly, and C. Bezant, Measured and calculated elastic wave velocities for xenoliths from the lower crust and upper mantle, Tectonophys., 173, 207-210, 1990.

Jaeger, J., and N. Cook, Fundamentals of Rock Mechanics, Chapman and Hall, London, 1979.

Jaoul, O., J. Tullis, and A. Kronenberg, The effect of varying water contents on the creep behavior of Heavitree quartzite, J. Geophys. Res., 89, 4298-4312, 1984.

Jemsek, J., E. Bergman, J. Nabelek, and S. Solomon, Focal depths and mechanisms of large earthquakes on the Arctic mid-ocean ridge system, J. Geophys. Res., 91, 13,993-14,005, 1986.

Kanamori, H., and D. Anderson, Theoretical basis of some empirical relations in seismology, Bull. Seis. Soc. Am., 65, 1073-1095, 1975.

Kane, K., and D. Hayes, Tectonic corridors in the South Atlantic: Evidence for long-lived midocean ridge segmentation, J. Geophys. Res., 97, 17,317-17,330, 1992.

Kane, K., and D. Hayes, Long-lived mid-ocean ridge segmentation: Constraints on models, $J$. Geophys. Res., 99, 19,693-19,706, 1994.

Karson, J., and H. Dick, Tectonics of ridge-transform intersections at the Kane fracture zone, Mar. Geophys. Res., 6, 51-98, 1983. 
Karson, J., and A. Winters, Along-axis variations in tectonic extension and accommodation zones in the MARK Area, Mid-Atlantic Ridge $23^{\circ} \mathrm{N}$ latitude, in Ophiolites and their Modern Oceanic Analogues, edited by L. Parson, B. Murton, and P. Browning, pp. 107-116, Geol. Soc. of London, 1992.

Karson, J., et al., Along-axis variations in seafloor spreading in the MARK area, Nature, 328, $681-685,1987$.

Kay, R., and S. Kay, The nature of the lower continental crust: Inferences from geophysics, surface geology, and crustal xenoliths, Rev. Geophys., 19, 271-297, 1981.

Kelemen, P., and W Holbrook, Origin of thick, high-velocity igneous crust along the U.S. East Coast margin, J. Geophys. Res., 100, 10,077-10,094, 1995.

Keller, G., P. Morgan, and W. Seager, Crustal structure, gravity anomalies and heat flow in the southern Rio Grande rift and their relationship to extensional tectonics, Tectonophys., 174, 21-37, 1990.

Kern, $\mathrm{H}$., $\mathrm{P}$ - and S-wave velocities in crustal and mantle rocks under the simultaneous action of high confining pressure and high temperature and the effect of rock microstructure, in HighPressure Researches in Geoscience, edited by W. Schreyer, pp. 15-45, Schweizerbart'sche, 1982.

Kern, H., and A. Richter, Temperature derivatives of compressional and shear wave velocities in crustal and mantle rocks at 6 kbar confining pressure, J. Geophys., 49, 47-56, 1981.

Kern, H., and V. Schenk, Elastic wave velocities in rocks from a lower crustal section in southern Calabria (Italy), Phys. Earth Planet. Inter, 40, 147-160, 1985.

Kern, H., and V. Schenk, A model of velocity structure beneath Calabria, southern Italy, based on laboratory data, Earth Planet. Sci. Lett., 87, 325-337, 1988.

Kern, H., and J. Tubia, Pressure and temperature dependence of P- and S-wave velocities, seismic anistropy and density of sheared rocks from the Sierra Alpujata massif (Ronda peridotites, southern Spain), Earth Planet. Sci. Lett., 119, 191-205, 1993.

King, B., Structural and volcanic evolution of the Gregory Rift Valley, in Geological background to fossil man, edited by W. Bishop, pp. 29-54, Scottish Academic Press LTD, Edinburgh, 1978.

Kinlzer, R., and T. Grove, Corrections and further discussion of the primary magmas of mid-ocean ridge basalts, 1 and 2, J. Geophys. Res., 98, 22,339-22,347, 1993.

Kinzler, R., Melting of mantle peridotite at pressures approaching the spinel to garnet transition: Application to mid-ocean ridge basalt petrogenesis, J. Geophys. Res., 102, 853-874, 1997.

Kinzler, R., and T. Grove, Primary magmas of mid-ocean ridge basalts, 2. applications, J. Geophys. Res., 97, 6907-6926, 1992.

Kiratzi, A., A study of the active crustal deformation of the North and East Anatolian Fault Zones, Tectonophys., 225, 191-203, 1993.

Kirby, S., Rheology of the lithosphere, Rev. Geophys. and Space Phys., 21, 1458-1487, 1983. 
Klitgord, K., and J. Behrendt, Basin structure of the U.S. Atlantic margin, in Geological and Geophysical Investigations of Continental Margins, edited by J. Watkins, L. Montadert, and P. Dickerson, AAPG Memoir, 29 ed., pp. 85-112, 1979.

Klitgord, K., and H. Schouten, Plate kinematics of the central Atlantic, in The Geology of North America, edited by P. Vogt and B. Tucholke, vol. The Western North Atlantic Region, v. M, pp. 351-377, Geol. Soc. of Am., 1986.

Klitgord, K., D. Hutchinson, and H. Schouten, U.s. atlantic continental margin; Structure and tectonic framework, in The Atlantic Continental Margin, U.S., edited by R. Sheridan and J. Grow, vol. The Geology of North America, v. I-2, pp. 19-55, Geol. Soc. of Am., 1988.

Klitgord, K., C. Poag, C. Schneider, and L. North, Geophysical database of the East Coast of the United States: Northern Atlantic Margin: Cross sections and gridded database, Open File 94-637, USGS, 1994.

Kogiso, T., K. Hirose, and E. Takahashi, Melting experiments on homogeneous mixtures of peridotite and basalt: application to the genesis of ocean island basalts, Earth Planet. Sci. Lett., 162, 45-61, 1998.

Kohlstedt, D., B. Evans, and S. Mackwell, Strength of the lithosphere: Constraints imposed by laboratory experiments, J. Geophys. Res., 100, 17,587-17,602, 1995.

Kong, L., S. Solomon, and G. Purdy, Microearthquake characteristics of a Mid-Ocean Ridge alongaxis high, J. Geophys. Res., 97, 1659-1685, 1992.

Korenaga, J., and T. Jordan, On the state of sublithospheric upper mantle beneath a supercontinent, Geophys. J. Int., in press, 2002.

Korenaga, J., W. Holbrook, G. Kent, P. Kelemen, R. Detrick, H.-C. Larsen, J. Hopper, and T. DahlJensen, Crustal structure of the southeast Greenland margin from joint refraction and reflection seismic tomography, J. Geophys. Res., 105, 21,591-21,614, 2000.

Korenaga, J., P. Kelemen, and W. Holbrook, Methods for resolving the origin of large igneous provinces from crustal seismology, J. Geophys. Res., submitted, 2002.

Kronenberg, A., and J. Tullis, Flow strength of quartz aggregates: Grain size and pressure effects due to hydrolytic weakening, J. Geophys. Res., 89, 4281-4297, 1984.

Kuo, B., and D. Forsyth, Gravity anomalies of the ridge-transform system in the South Atlantic between $31^{\circ}$ and $34.5^{\circ} \mathrm{S}$ : Upwelling centers and variations in crustal thickness, Mar. Geophys. Res., 10, 205-232, 1988.

Kuo, B., W. Morgan, and D. Forsyth, Asymmetry in topography of the crestal mountains near a ridge-transform intersection, EOS, Trans. AGU, 65, 274, 1984.

Kusznir, N., and R. Park, The extensional strength of the continental lithosphere: Its dependence on geothermal gradient, and crustal composition and thickness, in Continental Extensional Tectonics, Special Pub. No. 28, edited by M. Coward, J. Dewey, and P. Hancock, pp. 35-52, Geol. Soc. London, 1987. 
LASE Study Group, Deep structure of the U.S. East Coast passive margin from large aperture seismic experiments (LASE), Mar. Pet. Geol., 3, 234-242, 1986.

Lavier, L., W. Buck, and A. Poliakov, Factors controlling normal fault offset in an ideal brittle layer, J. Geophys. Res., 105, 23,431-23,442, 2000.

Lin, J., and E. Parmentier, A finite amplitude necking model of rifting in brittle lithosphere, $J$. Geophys. Res., 95, 4909-4923, 1990.

Lin, J., and J. Phipps Morgan, The spreading rate dependence of three-dimensional mid-ocean ridge gravity structure, Geophys. Res. Lett., 19, 13-16, 1992.

Lin, J., G. Purdy, H. Schouten, J.-C. Sempéré, and C. Zervas, Evidence from gravity data for focused magmatic accretion along the Mid-Atlantic Ridge, Nature, 344, 627-632, 1990.

Logatchev, N., and Y. Zorin, Baikal rift zone: Structure and geodynamics, Tectonophys., 208, 273-286, 1992.

Lysak, S., Terrestrial heat flow of continental rifts, Tectonophys., 143, 31-41, 1987.

Lysak, S., Heat flow variations in continental rifts, Tectonophys., 208, 309-323, 1992.

Macdonald, K., Mid-ocean ridges: Fine scale tectonic, volcanic and hydrothermal processes within the plate boundary zone, Ann. Rev. Earth Planet. Sci., 10, 155-190, 1982.

Macdonald, K., The crest of the Mid-Atlantic Ridge: Models for crustal generation processes and tectonics, in The Geology of North America, edited by P. Vogt and B. Tucholke, vol. The Western North Atlantic Region, v. M, pp. 51-68, Geol. Soc. Am., 1986.

Macdonald, K., and T. Atwater, Evolution of rifted ocean ridges, Earth Planet. Sci. Lett., 39, 319-327, 1978.

Macdonald, K., D. Castillo, S. Miller, P. Fox, K. Kastens, and E. Bonatti, Deep-tow studies of the Vema Fracture Zone 1. Tectonics of a major slow slipping transform fault and its intersection with the Mid-Atlantic Ridge, J. Geophys. Res., 91, 3334-3354, 1986.

Mackwell, S., M. Zimmerman, and D. Kohlstedt, High-temperature deformation of dry diabase with application to tectonics on Venus, J. Geophys. Res., 103, 975-984, 1998.

Magde, L., and D. Sparks, Three-dimensional mantle upwelling, melt generation, and melt migration beneath segment slow spreading ridges, J. Geophys. Res., 102, 20,571-20,583, 1997.

Maguire, P., C. Swain, R. Masotti, and M. Khan, A crustal and uppermost mantle cross-sectional model of the Kenya rift derived from seismic and gravity data, Tectonophys., 236, 217-249, 1994.

Makris, J., and A. Ginzburg, The Afar depression: transition between continental rifting and seafloor spreading, Tectonophys., 141, 199-214, 1987.

Malinverno, A., and P. Cowie, Normal faulting and the topographic roughness of mid-ocean ridge flanks, J. Geophys. Res., 98, 17,921-17,939, 1993. 
Manghnani, M., R. Ramananantoadndro, and J. Clark, S.P., Compressional and shear wave velocities in granulite facies rocks and eclogites to $10 \mathrm{kbar}, J$. Geophys. Res., 79, 5427-5446, 1974.

Mayer, G., et al., The deep crust of the southern Rhine Graben: Reflectivity and seismicity as images of dynamic processes, Tectonophys., 275, 15-40, 1997.

McKenzie, D., Some remarks on the development of sedimentary basins, Earth Planet. Sci. Lett., 40, 25-32, 1978.

McKenzie, D., and M. Bickle, The volume and composition of melt generated by extension of the lithosphere, J. Petrol., 29, 625-679, 1988.

McLennan, S., and S. Taylor, Heat flow and the chemical composition of the continental crust, $J$. Geol., 104, 377-396, 1996.

Melosh, H., and A. Raefsky, A simple and efficient method for introducing faults into finite element computations, Bull. Seis. Soc. Am., 71, 1391-1400, 1981.

Melosh, H., and J. Williams, C.A., Mechanics of graben formation in crustal rocks: A finite element analysis, J. Geophys. Res., 94, 13,961-13,973, 1989.

Mendel, V., D. Sauter, L. Parson, and J.-R. Vanney, Segmentation and morphotectonic variations along a super slow-spreading center: The Southwest Indian Ridge $\left(57^{\circ} \mathrm{E}-70^{\circ} \mathrm{E}\right)$, Mar. Geophys. Res., 19, 505-533, 1997.

Miller, D., and N. Christensen, Seismic signature and geochemistry of an island arc: A multidisciplinary study of the Kohistan accreted terrane, northern Pakistan, J. Geophys. Res., 99, 11,62311,642, 1994.

Miller, D., and N. Christensen, Seismic velocities of the lower crustal and upper mantle rocks from the slow spreading Mid-Atlantic Ridge, south of the Kane transform zone (MARK), in Proc. Ocean Drill. Program Sci. Results, vol. 153, pp. 437-454, College Station, TX, 1997.

Miller, D., G. Iturrino, and N. Christensen, Geochemical and petrological constraints on velocity behavior of lower crustal and upper mantle rocks from the fast-spreading ridge at Hess Deep, in Proc. Ocean Drill. Program, Sci. Res., edited by C. Mével, K. Gillis, J. Allan, and P. Meyer, vol. 147, pp. 477-490, College Station, TX, 1996.

Minshull, T., and R. White, Thin crust on the flanks of the slow-spreading Southwest Indian Ridge, Geophys. J. Int., 125, 139-148, 1996.

Montési, L., Localization instability and origin of regularly spaced faults in planetary lithospheres, Ph.D. thesis, Massachusetts Institute of Techology, Cambridge, Massaschusetts, 2001.

Montési, L., and M. Zuber, A unified description of localization for application to large-scale tectonics, J. Geophys. Res., 107, 2002.

Morgan, P., Heat flow in rift zones, in Continental and Oceanic Rifts, edited by G. Palmason, vol. Geodynamic Series 8, pp. 107-122, Am. Geophys. Union, 1982. 
Morozov, I., S. Smithson, L. Hollister, and J. Diebold, Wide-angle seismic imaging across accreted terranes, southeastern Alaska and western British Columbia, Tectonophys., 299, 281-296, 1998.

Moustafa, A., Internal structure and deformation of an accommodation zone in the northern part of the Suez rift, J. Struc. Geol., 18, 93-107, 1996.

Muller, M., T. Minshull, and R. White, Segmentation and melt supply at the Southwest Indian Ridge, Geology, 27, 867-870, 1999.

Mutter, J., and J. Karson, Structural process at slow-spreading ridges, Science, 257, 627-634, 1992.

Mutter, J., M. Talwani, and P. Stoffa, Evidence for a thick oceanic crust adjacent to the Norwegian margin, J. Geophys. Res., 89, 483-502, 1984.

Mutter, J., W. Buck, and C. Zehnder, A model for the formation of thick basaltic sequences during the initiation of spreading, J. Geophys. Res., 93, 1031-1048, 1988.

Neumann, G., and M. Zuber, A continuum approach to the development of normal faults, in Proc. 35th US Symposium on Rock Mechanics, edited by J. Daemen and R. Schultz, pp. 191-198, Balkema, Lake Tahoe, Nevada, 1995.

Okada, Y., Internal deformation due to shear and tensile faults in a half-space, Bull. Seis. Soc. Am., 82, 1018-1040, 1992.

Okal, E., and A. Langenhorst, Seismic properties of the Etanin Transform System, South Pacific, Phys. Earth Planet. Int., 8119, 185-208, 2000.

Pakiser, L., and R. Robinson, Composition and evolution of the continental crust as suggested by seismic observations, Tectonophys., 3, 547-557, 1966.

Pallister, J., Parent magmas of the Semail ophiolite, Oman, in Ophiolites and Oceanic Lithosphere, edited by I. Gass, S. Lippard, and A. Shelton, vol. 13, pp. 63-70, Geol. Soc. Spec. Pub., 1984.

Pariso, J., J.-C. Sempéré, and C. Rommevaux, Temporal and spatial variations in crustal accretion along the Mid-Atlantic Ridge $\left(29^{\circ}-31^{\circ} 30^{\prime} \mathrm{N}\right)$ over the last $10 \mathrm{~m}$.y.: Implications from a threedimensional gravity study, J. Geophys. Res., 100, 17,781-17,794, 1995.

Parker, R., The rapid calculation of potential anomalies, Geophys. J. R. Astron. Soc., 31, 447-455, 1972.

Parmentier, E., and J. Phipps Morgan, Spreading rate dependence of three-dimensional structure in oceanic spreading centres, Nature, 348, 325-328, 1990.

Patton, T., A. Moustafa, R. Nelson, and S. Abdine, Tectonic evolution and structural setting of the Suez rift, in Interior Rift Basins, edited by S. Landon, AAPG Memoir 59, pp. 9-55, 1994.

Pederson, R., J. Malpas, and T. Falloon, Petrology and geochemistry of gabbroic and related rocks from Site 894, Hess Deep, in Proc. Ocean Drill. Program, Sci. Res., edited by C. Mével, K. Gillis, J. Allan, and P. Meyer, vol. 147, pp. 3-19, College Station, TX, 1996. 
Phipps Morgan, J., and Y. Chen, The genesis of oceanic crust: Magma injection, hydrothermal circulation, and crustal flow, J. Geophys. Res., 98, 6283-6297, 1993.

Phipps Morgan, J., and D. Forsyth, Three-dimensional flow and temperature perturbations due to a transform offset: Effects on oceanic crust and upper mantle structure, J. Geophys. Res., 93, 2955-2966, 1988.

Phipps Morgan, J., and E. Parmentier, Lithospheric stress near a ridge-transform intersection, Geophys. Res. Lett., 11, 113-116, 1984.

Phipps Morgan, J., E. Parmentier, and J. Lin, Mechanisms for the origin of mid-ocean ridge axial topography: Implications for the thermal and mechanical structure of accreting plate boundaries, J. Geophys. Res., 92, 12,823-12,836, 1987.

Plouff, D., Gravity and magnetic fields of polygonal prisms and application to magnetic terrain corrections, Geophysics, 41, 727-741, 1976.

Pockalny, R., A. Smith, and P. Gente, Spatial and temporal variability of crustal magnetization of a

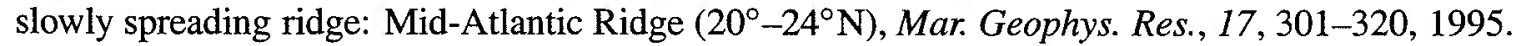

Pockalny, R., P. Gente, and R. Buck, Oceanic transverse ridges: A flexural response to fracturezone-normal extension, Geology, 24, 71-74, 1996.

Poliakov, A., and W. Buck, Mechanics of strectching elastic-plastic-viscous layers: Applications to slow-spreading mid-ocean ridges, in Faulting and Magmatism at Mid-Ocean Ridges, Geophys. Mono. Ser, edited by W. Buck, P. Delaney, J. Karson, and Y. Lagabrielle, vol. 106, pp. 305-323, AGU, Washington, D.C., 1998.

Pollack, H., and D. Chapman, On the regional variation of heat flow, geotherms, and lithospheric thickness, Tectonophys., 38, 279-296, 1977.

Pollard, D., and A. Aydin, Propagation and linkage of oceanic ridge segments, J. Geophys. Res., $89,10,017-10,028,1984$.

Poort, J., P van der Beek, and M. ter Voorde, An integrated modelling study of the central and northern Baikal rift: Evidence for non-uniform lithospheric thinning?, Tectonophys., 291, 101$122,1998$.

Prodehl, C., S. Mueller, A. Glahn, M. Gutscher, and V. Haak, Lithospheric cross sections of the European Cenozoic rift system, Tectonophys., 208, 113-138, 1992.

Purdy, G., J.-C. Sempéré, H. Schouten, D. Dubois, and R. Goldsmith, Bathymetry of the MidAtlantic Ridge, $24^{\circ}-31^{\circ} \mathrm{N}$ : A map series, Mar. Geophys. Res., 12, 247-252, 1990.

Rabain, A., M. Cannat, J. Escartín, G. Pouliquen, C. Deplus, and C. Rommevaux-Jestin, Focused volcanism and growth of a slow-spreading segment (Mid-Atlantic Ridge, $35^{\circ} \mathrm{N}$ ), Earth Planet. Sci. Lett., 185, 211-224, 2001.

Rabinowicz, E., Friction and Wear of Materials, John Wiley, New York, 1965.

Raitt, R., The crustal rocks, in The Sea, edited by M. Hill, pp. 85-102, Wiley-Interscience, 1963. 
Reddy, J., An Introduction to the Finite Element Method, McGraw-Hill, New York, 1984.

Reid, I., Crustal structure of a nonvolcanic rifted margin east of Newfoundland, J. Geophys. Res., $99,15,161-15,180,1994$.

Roest, W., J. Dañobeitia, J. Verhoef, and B. Collette, Magnetic anomalies in the Canary Basin and the Mesozoic evolution of the Central North Atlantic, Mar. Geophys. Res., 14, 1-24, 1992.

Rowlett, H., and D. Forsyth, Recent faulting and microearthquakes at the intersection of the Vema Fracture Zone and the Mid-Atlantic Ridge, J. Geophys. Res., 89, 6079-6094, 1984.

Rudnick, R., and D. Fountain, Nature and composition of the continental crust: A lower crustal perspective, Rev. Geophys., 33, 267-309, 1995.

Rudnick, R., and T. Presper, Geochemistry of intermediate- to high-pressure granulites, in Granulites and Crustal evolution, edited by D. Vielzeuf and P. Vidal, pp. 523-550, Kluwer Acad. Pub., Netherlands, 1990.

Rudnicki, J., and M. Wu, Mechanics of dip-slip faulting in an elastic half-space, J. Geophys. Res., 100, 22,173-22,186, 1995.

Ruina, A., Slip instability and state variable friction laws, J. Geophys. Res., 88, 10,359-10,370, 1983.

Rutter, E., and K. Brodie, Rheology of the lower crust, in Continental Lower Crust, Dev. Geotecton., edited by D. Fountain and R. Arculus, pp. 201-267, Elsevier, New York, 1992.

Salisbury, M., and N. Christensen, The seismic velocity structure of a traverse through the Bay of Islands ophiolite complex, Newfoundland, and exposure of oceanic crust and upper mantle, $J$. Geophys. Res., 83, 805-817, 1984.

Sandwell, D., and W. Smith, Marine gravity anomaly from Geosat and ERS 1 satellite altimetry, J. Geophys. Res., 102, 10,039-10,054, 1997.

Sawyer, D., Total tectonic subsidence: A parameter for distinguishing crust type at the U.S. Atlantic Continental Margin, J. Geophys. Res., 90, 7751-7769, 1985.

Scholz, C., The Mecahnics of Earthquakes and Faulting, Cambridge Univ. Press, New York, 1990.

Searle, R., GLORIA survey of the East Pacific Rise near $3.5^{\circ} \mathrm{S}$ : Tectonic and volcanic characteristics of a fast spreading mid-ocean rise, Tectonophys., 101, 319-344, 1984.

Searle, R., and A. Laughton, Fine-scale sonar study of tectonics and volcanism on the Reykjanes Ridge, Oceanol. Acta, 4 suppl., 5-13, 1981.

Searle, R., P. Cowie, N. Mitchell, S. Allerton, C. MacLeod, J. Escartín, S. Russell, P. Slootweg, and T. Tanaka, Fault structure and detailed evolution of a slow spreading ridge segment: the MidAtlantic Ridge at $29^{\circ} \mathrm{N}$, Earth Planet. Sci. Lett., 154, 167-183, 1998.

Sempéré, J.-C., J. Lin, H. Brown, H. Schouten, and G. Purdy, Segmentation and morphotectonic variations along a slow-spreading center: The Mid-Atlantic ridge $\left(24^{\circ} 00^{\prime} \mathrm{N}-30^{\circ} 40^{\prime} \mathrm{N}\right), \mathrm{Mar}$. Geophys. Res., 15, 153-200, 1993. 
Sempéré, J.-C., et al., The Mid-Atlantic Ridge between $29^{\circ} \mathrm{N}$ and $31^{\circ} 30^{\prime} \mathrm{N}$ in the last $10 \mathrm{Ma}$, Earth Planet. Sci. Lett., 130, 45-55, 1995.

Severinghaus, J., and K. Macdonald, High inside corners at ridge-transform intersections, Mar. Geophys. Res., 9, 353-367, 1988.

Shaw, D., J. Cramer, M. Higgins, and M. Truscott, Composition of the Canadian Precambrian shield and the continental crust of the earth, in The Nature of the Lower Continental Crust, edited by J. Dawson, D. Carswell, J. Hall, and K. Wedepohl, pp. 275-282, Geol. Soc. Spec. Pub. No. 24, 1986.

Shaw, P., Ridge segmentation, faulting and crustal thickness in the Atlantic Ocean, Nature, 358, 490-493, 1992.

Shaw, P., and J. Lin, Causes and consequences of variations in faulting style at the Mid-Atlantic Ridge, J. Geophys. Res., 98, 21,839-21,851, 1993.

Shaw, W., and J. Lin, Models of ocean ridge lithospheric deformation: Dependence on crustal thickness, spreading rate, and segmentation, J. Geophys. Res., 101, 17,977-17,993, 1996.

Shor, J., G.G., H. Menard, and R. Raitt, Structure of the Pacific Basin, in The Sea, edited by A. Maxwell, pp. 3-27, Wiley-Interscience, 1970.

Simpson, R., R. Jachens, R. Blakely, and R. Saltus, A new isostatic residual gravity map of the conterminous United States with a discussion on the significance of isostatic residual anomalies, J. Geophys. Res., 91, 8348-8372, 1986.

Sinno, Y., P. Daggett, G. Keller, P. Morgan, and S. Harder, Crustal structure of the southern Rio Grande rift determined from seismic refraction profiling, J. Geophys. Res., 91, 6143-6156, 1986.

Smith, D., M. Tolstoy, C. Fox, D. Bohnenstiehl, M. Cannat, J. Escartín, and S. Bazin, Spatial and temporal distribution of seismicity along the northern Mid-Atlantic Ridge, 15-35N, from an autonomous hydrophone array, Eos. Trans. AGU, 82(47), Fall Meet. Suppl., Abstract S21A-0555, 2001.

Smith, D., et al., Mid-Atlantic Ridge volcanism from deep-towed side-scan sonar images, $25^{\circ}-$ $29^{\circ} \mathrm{N}$, J. Volc. Geotherm. Res., 67, 233-262, 1995.

Sobolev, S., and A. Babeyko, Modeling of mineralogical composition, density and elastic wave velocities in anhydrous magmatic rocks, Surv. in Geophys., 15, 515-544, 1994.

Solomon, S., P. Huang, and L. Meinke, The seismic moment budget of slowly spreading ridges, Nature, 334, 58-60, 1988.

Sonder, L., and P England, Effects of a temperature-dependent rheology on large-scale continental extension, J. Geophys. Res., 94, 7603-7619, 1989.

Spyropoulos, C., W. Griffith, C. Scholz, and B. Shaw, Experimental evidence for different strain regimes of crack populations in a clay model, Geo. Res. Lett., 26, 1081-1084, 1999. 
Steckler, M., S. Feinstein, B. Kohn, L. Lavier, and M. Eyal, Pattern of mantle thinning from subsidence and heat flow measurements in the Gulf of Suez: Evidence for the rotation of Sinai and along-strike flow from the Red Sea, Tectonics, 17, 903-920, 1998.

Stein, C., and S. Stein, A model for the global variation in oceanic depth and heat flow with lithospheric age, Nature, 359, 123-129, 1992.

Stein, R., and T. Hanks, $M \geq 6$ Earthquakes in southern California during the 20th Century: No evidence for a seismicity or moment deficit, Bull. Seismol. Soc. Amer., 88, 635-652, 1998.

Suyehiro, K., et al., Continental crust, crustal underplating, and low-Q upper mantle beneath an oceanic island arc, Science, 272, 390-392, 1996.

Talwani, M., J. Ewing, R. Sheridan, W. Holbrook, and I. Glover, L., The Edge Experiment and the U.S. East Coast Magnetic Anomaly, in Rifted Ocean-Continental Boundaries, edited by E. Banda, M. Torne, and M. Talwani, pp. 155-181, Kluwer Acad., Norwell, Mass., 1995.

Tapponnier, P., and J. Francheteau, Necking of the lithosphere and the mechanics of slowly accreting plate boundaries, J. Geophys. Res., 83, 3955-3970, 1978.

Taylor, S., and S. McLennan, The composition and evolution of the continental crust: rare earth element evidence from sedimentary rocks, Phil. Trans. R. Soc. Lond., 301, 381-399, 1981.

Taylor, S., and S. McLennan, The continental crust: its composition and evolution, Blackwell, Oxford, 1985.

Thatcher, W., and D. Hill, A simple model for the fault-generated morphology of slow-spreading mid-oceanic ridges, J. Geophys. Res., 100, 561-579, 1995.

Thibaud, R., P. Gente, and M. Maia, A systematic analysis of the Mid-Atlantic Ridge morphology and gravity between $15^{\circ} \mathrm{N}$ and $40^{\circ} \mathrm{N}$ : Constraints of the thermal structure, J. Geophys. Res., 103, 24,223-24,243, 1998.

Tolstoy, M., A. Harding, and J. Orcutt, Crustal thickness on the Mid-Atlantic Ridge: Bull's-eye gravity anomalies and focused accretion, Science, 262, 726-729, 1993.

Toomey, D., S. Solomon, and G. Purdy, Microearthquakes beneath the median valley of the MidAtlantic Ridge near $23^{\circ} \mathrm{N}$ : Hypocenters and focal mechanisms, J. Geophys. Res., 93, 9093-9112, 1985.

Tréhu, A., A. Ballard, L. Dorman, J. Gettrust, K. Klitgord, and A. Schreiner, Structure of the lower crust beneath the Carolina Trough, U.S. Atlantic continental margin, J. Geophys. Res., 94, $10,585-10,600,1989 a$.

Tréhu, A., K. Klitgord, D. Sawyer, and R. Buffler, Atlantic and Gulf of Mexico continental margins, in Geophysical Framework of the Continental United States, edited by L. Pakiser and W. Mooney, Mem. Geol. Soc. of Am., 172 ed., pp. 349-383, Boulder, Colorado, 1989b.

Tucholke, B., and J. Lin, A geological model for the structure of ridge segments in slow spreading ocean crust, J. Geophys. Res., 99, 11,937-11,958, 1994. 
Tucholke, B., and H. Schouten, Kane Fracture Zone, Mar. Geophys. Res., 10, 1-39, 1988.

Tucholke, B., R. Houtz, and W. Ludwig, Sediment thickness and depth to basement in western North Atlantic Ocean basin, AAPG Bull., 66, 1384-1395, 1982.

Tucholke, B., J. Austin, J.A., and E. Uchupi, Crustal structure and rift-drift evolution of the Newfoundland Basin, in Extensional Tectonics and Stratigraphy of the North Atlantic Margin, edited by A. Tankard and H. Balkwell, AAPG Memoir, 46 ed., pp. 247-263, 1989.

Tucholke, B., J. Lin, M. Kleinrock, M. Tivey, T. Reed, J. Goff, and G. Jaroslow, Segmentation and crustal structure of the western Mid-Atlantic Ridge flank, $25^{\circ} 25^{\prime}-27^{\circ} 10^{\prime} \mathrm{N}$ and $0-29$ m.y., J. Geophys. Res., 102, 10,203-10,223, 1997.

Tuckwell, G., J. Bull, and D. Sanderson, Numerical models of faulting at oblique spreading centers, J. Geophys. Res., 103, 15,473-15,482, 1998.

Tullis, J., and R. Yund, Hydrolytic weakening of experimentally deformed Westerly granite and Hale albite rock, J. Struct. Geol., 2, 439-451, 1980.

Tullis, T., and J. Weeks, Constitutive behavior and stability of frictional sliding of granite, $\mathrm{Pa}$ geoph., 124, 383-414, 1986.

Uchupi, E., J. Phillips, and K. Prada, Origin and structure of the New England Seamount Chain, Deep Sea Res., 17, 483-494, 1970.

Upcott, N., R. Mukasa, and C. Ebinger, Along-axis segmentation and isostasy in the Western rift, East Africa, J. Geophys. Res., 101, 3247-3268, 1996.

Vening Meinesz, F., Les grabens africains résultant de compression ou de tension dans la croûte terrestre?, Inst. R. Kolonial Belge Bull., 21, 539-552, 1950.

Verhoef, J., B. Collette, J. Daobeitia, H. Roeser, and W. Roest, Magnetic anomalies off WestAfrica $\left(20-30^{\circ} \mathrm{N}\right)$, Mar. Geophys. Res., 13, 81-103, 1991.

Verhoef, J., W. Roest, R. Macnab, and J. Arkani-Hamed, Magnetic anomalies of the Arctic and North Atlantic Oceans and adjacent land areas, Open File 3125, Geo. Surv. Canada, 1996.

Vogt, P., Early events in the opening of the North Atlantic, in Implications of Continental Drift to the Earth Sciences, edited by D. Tarling and S. Runcorn, pp. 693-712, Acad., San Diego, Calif., 1973.

Von Herzen, R., and V. Vacquier, Terrestrial heat flow in Lake Malawi, Africa, J. Geophys. Res., 72, 4221-4226, 1967.

Walsh, J., and J. Watterson, Distributions of cumulative displacement and seismic slip on a single normal fault surface, J. Struct. Geol., 9, 1039-1046, 1987.

Walter, M., Melting of garnet peridotite and the origin of komatiite and depleted lithosphere, $J$. Petrol., 39, 29-60, 1998.

Watt, J., G. Davies, and R. O'Connell, The elastic properties of composite materials, Rev. Geophys., 14, 541-563, 1976. 
Watts, A., and C. Marr, Gravity anomalies and the thermal and mechanical structure of rifted continental margins, in Rifted Ocean-Continental Boundaries, edited by E. Banda, M. Torne, and M. Talwani, pp. 65-94, Kluwer Acad., Norwell, Mass., 1995.

Watts, A., and J. Stewart, Gravity anomalies and segmentation of the continental margin offshore West Africa, Earth Planet. Sci. Lett., 156, 239-252, 1998.

Watts, A., J. Bodine, and M. Steckler, Observations of flexure and the state of stress in the oceanic lithosphere, J. Geophys. Res., 85, 6369-6376, 1980.

Weaver, B., and J. Tarney, Major and trace element composition of the continental lithosphere, Phys. Chem. Earth, 15, 39-68, 1984.

Weiland, C., K. Macdonald, and N. Grindlay, Ridge segmentation and the magnetic structure of the Southern Mid-Atlantic Ridge $26^{\circ} \mathrm{S}$ and $31^{\circ}-35^{\circ} \mathrm{S}$ : Implications for magmatic processes at slow spreading centers, J. Geophys. Res., 101, 8055-8073, 1996.

Wenzel, F., J.-P. Brun, and ECORS-DEKORP Working Group, A deep reflection seismic line across the northern Rhine Graben, Earth Planet. Sci. Lett., 104, 140-150, 1991.

Wheildon, J., P. Morgan, K. Williamson, T. Evans, and C. Swanberg, Heat flow in the Kenya rift zone, Tectonophys., 236, 131-149, 1994.

White, R., and D. McKenzie, Magmatism at rift zones: The generation of volcanic continental margins and flood basalts, J. Geophys. Res., 94, 7685-7729, 1989.

White, R., D. McKenzie, and R. O'Nions, Oceanic crustal thickness from seismic measurements and rare earth element inversions, J. Geophys. Res., 97, 19,683-19,715, 1992.

White, R., et al., Hatton Bank (northwest UK) continental margin structure, Geophys. J. R. Astron. Soc., 89, 265-271, 1987.

Whitehead, J., J.A., H. Dick, and H. Schouten, A mechanism for magmatic accretion under spreading centres, Nature, 312, 146-148, 1984.

Whitmarsh, R., et al., Drilling reveals transition from continental breakup to early magmatic crust, Eos Trans. AGU, 79, 173, 180-181, 1998.

Wiens, D., and S. Stein, Intraplate seismicity and stresses in young oceanic lithosphere, J. Geophys. Res., 89, 11,442-11,464, 1984.

Wilcock, W., G. Purdy, and S. Solomon, Microearthquake evidence for extension across the Kane Transform Fault, J. Geophys. Res., 95, 15,439-15,462, 1990.

Wolfe, C., E. Bergman, and S. Solomon, Oceanic transform earthquakes with unusual mechanisms or locations: Relation to fault geometry and state of stress in adjacent lithosphere, J. Geophys. Res., 98, 16,187-16,211, 1993.

Wolfe, C., G. Purdy, D. Toomey, and S. Solomon, Microearthquake characteristics and crustal velocity structure at $29^{\circ} \mathrm{N}$ on the Mid-Atlantic Ridge: The architecture of a slow spreading segment, J. Geophys. Res., 100, 24,449-24,472, 1995. 
Zuber, M., and E. Parmentier, Lithospheric necking: A dynamic model for rift morphology, Earth Planet. Sci. Lett., 77, 373-383, 1986.

Zuber, M., E. Parmentier, and R. Fletcher, Extension of continental lithosphere: A model for two scales of Basin and Range deformation, J. Geophys. Res., 91, 4826-4838, 1986. 